\title{
Influence of point mutations on the electro- physiological properties of a bacterial porin and its interaction with a $\beta$-lactam antibiotic
}

\author{
DISSERTATION \\ For the award of the degree \\ Doctor rerum naturalium \\ within the doctoral program \\ Physics of Biological and Complex Systems \\ of the Georg-August-University of Science (GAUSS)
}

Submitted by

Annika Bartsch

from Mainz 



\section{Thesis Committee:}

Prof. Dr. Claudia Steinem,

Institut für Organische und Biomolekulare Chemie,

Georg-August-Universität Göttingen

Prof. Dr. Ulf Diederichsen,

Institut für Organische und Biomolekulare Chemie,

Georg-August-Universität Göttingen

Prof. Dr. Michael Meinecke,

Institut für Zelluläre Biochemie,

Universitätsmedizin Göttingen

\section{Members of the Examination Board:}

Referee: Prof. Dr. Claudia Steinem,

Institut für Organische und Biomolekulare Chemie,

Georg-August-Universität Göttingen

$2^{\text {nd }}$ Referee: Prof. Dr. Ulf Diederichsen,

Institut für Organische und Biomolekulare Chemie, Georg-August-Universität Göttingen

\section{Further members of the Examination Board:}

Prof. Dr. Michael Meinecke,

Institut für Zelluläre Biochemie,

Universitätsmedizin Göttingen

Prof. Dr. Bert de Groot,

Computational Biomolecular Dynamics Group,

Max-Planck-Institut für Biophysikalische Chemie

Prof. Dr. Burkhard Geil,

Institut für Physikalische Chemie,

Georg-August-Universität Göttingen

Dr. Thomas Burg,

Biologische Mikro- und Nanotechnologie,

Max-Planck-Institut für Biophysikalische Chemie

Date of oral examination: $12^{\text {th }}$ July 2018 



\section{DECLARATION}

I, Annika Bartsch, hereby certify that my doctoral thesis entitled "Influence of point mutations on the electrophysiological properties of a bacterial porin and its interaction with a $\beta$ lactam antibiotic" has been written independently and with no other sources and aids than quoted.

Göttingen, 2018

Annika Bartsch 

Meiner Familie

„Fantasie ist wichtiger als Wissen, denn Wissen ist begrenzt." Albert Einstein 

ABSTRACT The high rate of complications and mortality in hospitals due to multidrug resistances (MDR) of gram-negative bacteria demonstrates the urgency to understand the underlying mechanisms. One common cause of MDR are mutations in bacterial porins, which are $\beta$-barrel proteins responsible for the uptake of antibiotics through the outer bacterial membrane. They have a periplasmic and an extracellular side, which are separated by the constriction region (CR) in the center of the pore. The CR is the narrowest point of the pore and a key region for antibiotic binding and permeation. Upon mutations in this region, the interaction between porin and antibiotic can be altered, leading to an impaired antibiotic permeation and thus increasing resistance.

In this work, the influence of mutations of the major porin PorB of Neisseria meningitidis on the interaction with the $\beta$-lactam antibiotic ampicillin was investigated using the planar patch-clamp technique supported by molecular dynamics (MD) simulations. In electrophysiology, the interaction between channel and substrate can be detected by short blockages of the open channel conductance, caused by a bound molecule. Examining the mutants G103K and G103D allowed analyzing the effect of a large residue and an additional positive (G->K) or negative charge (G->D) at the CR on the electrophysiological properties of the pores and the interaction with ampicillin. First, the electrophysiological properties of PorB wild type (wt) and the mutants were characterized in absence of ampicillin. A strong reduction of the open channel conductance upon the mutation G->K was found, whereas the mutation G->D only had a small influence. The alteration of the pore's inner electric field due to the mutation $\mathrm{G}->\mathrm{K}$ is a probable cause for this finding. The open channel noise, however, was strongly increased due to the mutation G->D, supposedly due to additional electrostatic repulsions of the negative charges at the CR.

The electrophysiological measurements in the presence of ampicillin showed an interaction of ampicillin with PorB wt and the mutant G103K, but not with the mutant G103D, which can be rationalized by a destabilization of the ampicillin binding site due to the increased electrostatic repulsion at the CR. Thus, the interaction with ampicillin was only compared between PorB wt and PorB G103K.

Ampicillin blocks the PorB channels only when added to their extracellular sides. Together with the asymmetric location of the binding site at the extracellular side found by MD simulations, this suggests a high energy barrier for ampicillin entering from the periplasmic side. Ampicillin is bound longer in the G103K pore than in the PorB wt pore and is more often released back to the extracellular lumen, indicating a higher energy barrier for ampicillin permeation through the mutant than through the wild type. This is ascribed to the different orientations ampicillin adopts upon entering the CR of the two porins. Hence, the mutation G->K substantially alters the interaction between PorB and ampicillin and probably reduces its permeation. 

ZUSAMMENFASSUNG Die hohe auf multiresistente Keime zurückzuführende Komplikations- und Sterblichkeitsrate in Krankenhäusern verdeutlicht die Dringlichkeit, die zugrunde liegenden Mechanismen zu verstehen. Eine häufige Ursache von Multiresistenzen sind Mutationen von Porinen, welche für den Transport von Antibiotika in die Zellen von gram-negativen Bakterien verantwortlich sind. Porine sind $\beta$-Fässer mit einem peripasmischen und einem extrazellulären Zugang, welche durch die sogenannte Konstriktionszone (constriction region, CR) in der Mitte der Pore getrennt sind. Die CR ist die engste Stelle der Pore und spielt eine entscheidende Rolle in Bezug auf Anbindung und Transport von Antibiotika. Durch Mutationen in dieser Region kann die Wechselwirkung zwischen Antibiotikum und Pore in einem Maß verändert werden, welches den Transport beeinträchtigt und somit die Resistenz des Bakteriums erhöht.

Im Rahmen dieser Arbeit wurde der Einfluss von Mutationen des Porins PorB aus Neisseria meningitidis auf die Wechselwirkung mit dem $\beta$-lactam Antibiotikum Ampicillin mittels planarer Patch-Clamp Technik untersucht und durch Molekulardynamik (MD) Simulationen unterstützt. In elektrophysiologischen Messungen kann die Wechselwirkung zwischen Kanal und Substrat durch kurze Blockaden des Kanalstroms, verursacht durch ein angebundenes Substrat, detektiert werden. Mittels der Mutanten G103K und G103D konnte der Effekt eines großen Restes und einer zusätzlichen positiven (G->K) oder negativen (G->D) Ladung in der CR auf die elektrophysiologischen Eigenschaften der Pore und die Interaktion mit Ampicillin untersucht werden.

Zunächst wurden die elektrophysiologischen Eigenschaften von PorB Wildtyp (wt) und den Mutanten ohne Ampicillin charakterisiert. Infolge der Mutation G->K wurde eine starke Verringerung der Kanalleitfähigkeit gefunden, wohingegen die Mutation G->D nur einen geringen Einfluss hat. Eine Modifikation des inneren elektrischen Feldes der Pore aufgrund der Mutation G->K ist die wahrscheinlichste Ursache. Ein erhöhtes Rauschen des offenen Kanals wurde hingegen nur bei der Mutante G103D detektiert und wird auf eine zusätzliche Abstoßung negativer Ladungen in der CR zurückgeführt.

Die elektrophysiologischen Messungen mit Ampicillin zeigten nur im Falle von PorB wt und G103K eine Blockade der Kanalleitfähigkeit, jedoch nicht für PorB G103D. Dies kann durch eine Destabilisierung der Bindungsstelle aufgrund der zusätzlichen Abstoßung der negativen Ladungen in der CR begründet werden. Entsprechend wurde die Wechselwirkung mit Ampicillin nur zwischen PorB wt und G103K verglichen.

Ampicillin blockiert den PorB Kanal nur, wenn es auf der extrazellulären Seite des Porins zugegeben wird. In Kombination mit der mittels MD Simulationen nachgewiesenen asymmetrischen Lage der Ampicillin-Bindungsstelle auf der extrazellulären Seite, spricht dies für eine hohe Energiebarriere für von der periplasmischen Seite eintretendes Ampicillin. In der Mutante ist Ampicillin länger gebunden und wird häufiger zurück zum extrazellulären Lumen entlassen. Dies lässt auf eine höhere Energiebarriere für die Permeation von Ampicillin durch G103K als durch PorB wt schließen und wird den unterschiedlichen Orientierungen, die Ampicillin beim Eintritt in die Konstriktionszone der beiden Porine einnimmt, zugeschrieben. Folglich beeinflusst die Mutation G->K die Wechselwirkung zwischen PorB und Ampicillin und vermindert vermutlich dessen Permeation durch das Porin. 



\section{ConTENT}

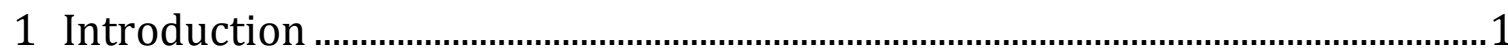

1.1 Structure and organization of the cell membrane....................................................

1.2 Transport through bacterial membranes: bacterial porins ......................................

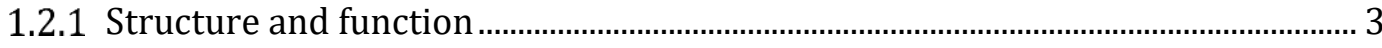

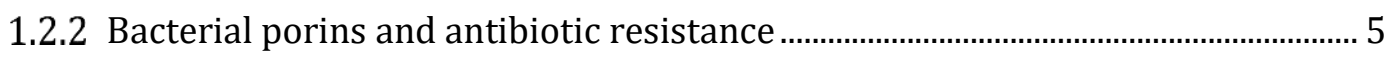

1.2.3 PorB from Neisseria meningitidis ............................................................................. 7

1.3 Investigation of antibiotic binding using the planar patch-clamp technique. 10

1.4 Interactions between bacterial porins and solutes ............................................ 11

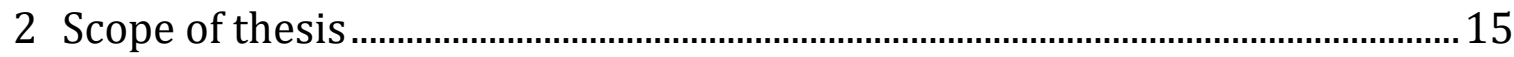

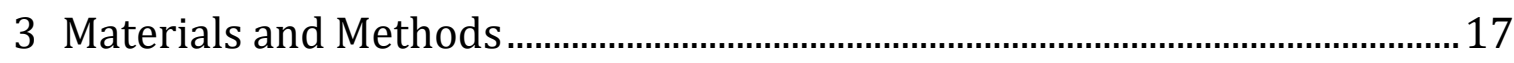

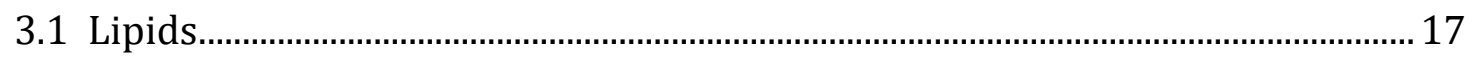

3.1.1 1,2-Diphytanoyl-sn-glycero-3-phosphocholine (DPhPC) .......................................17

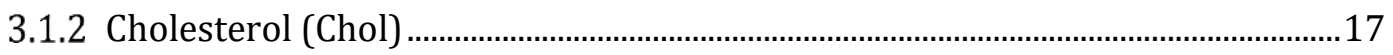

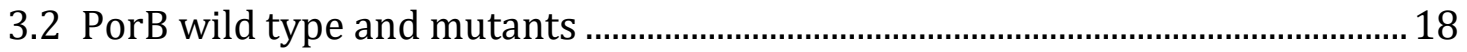

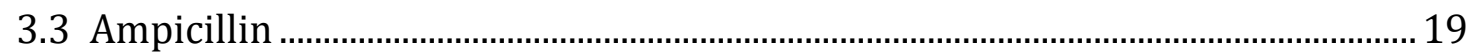

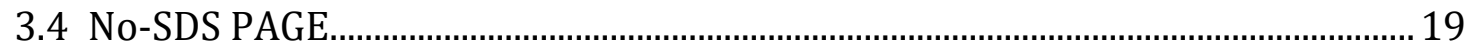

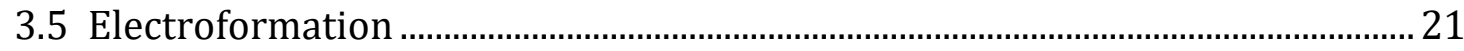

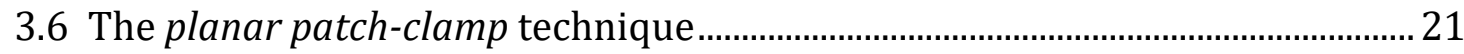

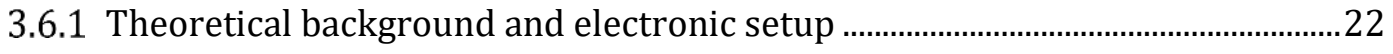

3.6.2 Membrane systems for planar patch-clamp ............................................................26

3.6.2.1 Solvent-free membranes ...........................................................................26

3.6.2.2 Black lipid membranes ……………………...............................................26

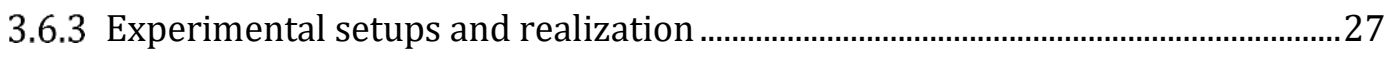

3.6.3.1 Solvent-free membranes ..............................................................................27

3.6.3.2 Black lipid membranes technique using the BLM setup..........................29 
3.6.3.3 Black lipid membranes technique using the Orbit 16

3.6.4 Data analysis

3.6.4.1 Single channel measurements without antibiotic 34

3.6.4.2 The analysis routine JULES 37

3.7 Molecular dynamics simulations 39

4 Results and Discussion 43

4.1 Characterization of the protein stock solutions using a no-SDS PAGE 43

4.2 Electrophysiological characterization in absence of ampicillin 44

4.2.1 Results of solvent-free planar patch-clamp measurements 45

4.2.1.1 Blank measurements 46

4.2.1.2 Conductivity and open probability of PorB wild type 48

4.2.1.3 Conductivity and open probability of PorB G103K. 50

4.2.1.4 Conductivity and open probability of PorB G103D 52

4.2.1.5 Comparison of PorB wild type and the mutants in solvent-free membranes 56

4.2.2 Results of black lipid membrane (BLM) measurements 62

4.2.2.1 Conductivity and open probability of PorB wild type 62

4.2.2.2 Conductivity and open probability of PorB G103K. 65

4.2.2.3 Conductivity and open probability of PorB G103D .67

4.2.2.4 Noise analysis 70

4.2.2.5 Comparison of PorB wild type and the mutants in BLMs 72

4.2.3 Comparison of PorB properties in solvent-free bilayers and BLMs 80

4.3 Investigation of the interaction with ampicillin. 85

4.3.1 Conductivity traces in the presence of ampicillin and power spectral densities 86

4.3.2 Analysis using the routine JULES 88

4.3.2.1 Blockage amplitude 89

4.3.2.2 Unilateral interaction and sign of the applied voltage 95 
4.3.2.3 Residence times of ampicillin.................................................................... 100

4.3.2.4 Blockage frequencies of ampicillin............................................................104

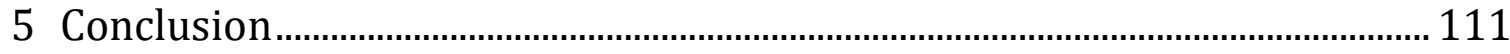

6 Literature

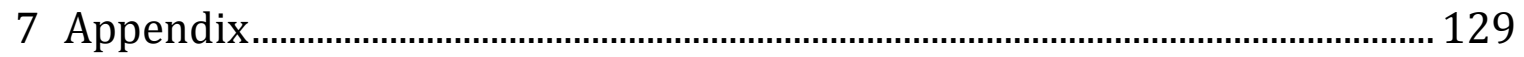

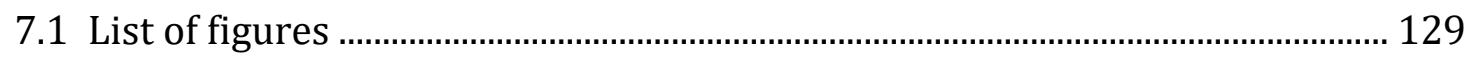

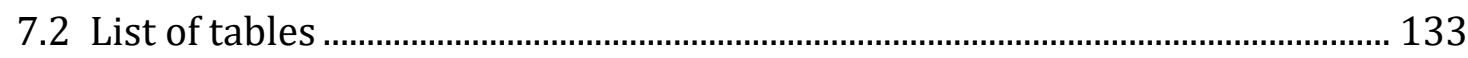

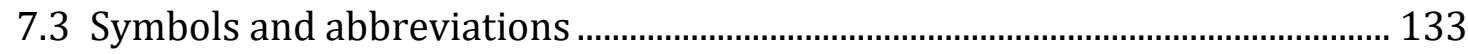

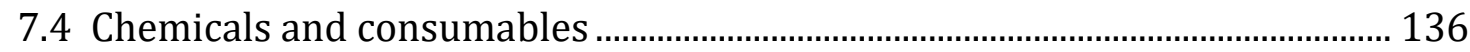

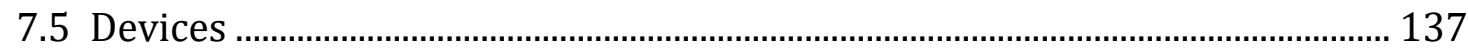

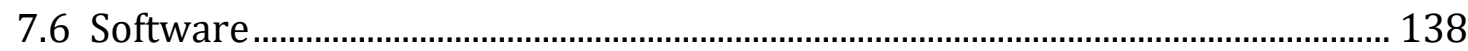

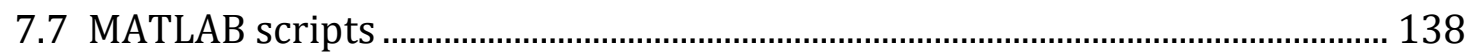

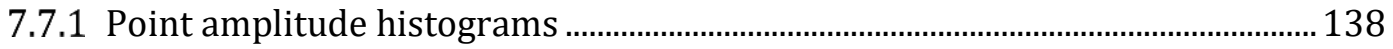

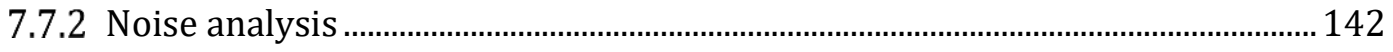





\section{INTRODUCTION}

\subsection{Structure and organization of the cell membrane}

Membranes play an important role in biological systems. Their main function is the separation of aqueous compartments, for example the separation of the cytosol from the environment.[1] The plasma membrane is selectively permeable for ions and organic molecules, thus preventing the cell from random in- and efflux of substances. In addition, many cellular processes involve the plasma membrane, such as signaling and metabolism. ${ }^{[2]}$

The organization of the cell membrane, consisting of phospholipids, glycolipids, sterols (in mammals: cholesterol) and proteins, is essential for its function and all membrane-associated processes.[3] Many models describing this organization were developed, but they are all based on the fluid mosaic model designed by Singer and Nicolson in 1972.[4] Due to the amphiphilic character of lipids and sterols a bilayer with a hydrophobic core is formed, exposing the polar lipid head groups to the aqueous surrounding and the cytosol, thereby avoiding the high energetic cost of a contact between hydrophilic and hydrophobic components. The proteins are either embedded into this lipid-matrix via transmembrane domains and denoted integral proteins, or they are peripheral proteins which can be attached to the membrane through a lipid anchor, electrostatic interactions with the lipid head groups or protein-protein interactions. ${ }^{[2]}$ If and to which extent a protein is integrated into the hydrophobic part of the bilayer depends on the distribution of its hydrophilic and hydrophobic regions, again to reach the energetic minimum. The membrane can be considered as a two-dimensional, oriented solution of integral proteins in the viscous bilayer of lipids. ${ }^{[4]}$ The fluidity of the mosaic structure allows transversal diffusion of the components, thus enabling redistribution and interaction between them. The model includes a short-range order mediated by protein-protein or protein-lipid interactions, resulting in defined aggregates. The long-range distribution of such aggregates and other membranes components, however, is explained to be random.

Although Singer and Nicolson described the general organization of the membrane quite well already in 1972, the research of the last decades provided further insights and lead to the development of further models. All of them deal with different aspects of the plasma membrane organization. ${ }^{[3]}$ 
The hydrodynamic model was first described in 1975 and deals with the mobility of transmembrane proteins, suggesting that molecular diffusion is primarily influenced by the viscosity and thickness of the membrane and not so much by the size of the diffusing particle..5] Modifying the model of lipid self-assembly and lipid domains lead to the concept of 'lipid rafts', formed by the clustering of sphingolipids and cholesterol. Lipid rafts are able to diffuse within a fluid bilayer and serve as platform for the attachment of certain proteins.[6]

The mattress model developed in 1984 focuses on the role of the hydrophobic mismatch between the proteins' hydrophobic part and the hydrophobic thickness of the bilayer and its influence on the formation of lipid/protein domains. ${ }^{[7]}$ The distortion of the lipids necessary to match the hydrophobic part of the proteins leads to local variation of the bilayer thickness. A possible 'sorting' of proteins and lipids induced by this effect is discussed to influence the nanoscale organization of the cell membrane.[3]

Engelman presented an updated version of the fluid mosaic model in 2005. ${ }^{[8]}$ The main refinement points include a variable patchiness, a variable thickness and a higher protein occupancy. The variable or functional patchiness suggests areas dominated by lipids and regions with functional protein complexes instead of a random long-range distribution. The implemented variable bilayer thickness results from the hydrophobic mismatch between proteins and lipids and can even cause membrane curvature. The higher protein occupancy includes the area occupied within the bilayer, as well as the surface area covered by proteins. Indeed, the plasma membrane of most animal cells consist of $50 \%$ protein. ${ }^{[9]}$ The area covered by proteins can be expected to be even larger, as many proteins and protein-complexes have large structures outside the bilayer.[8]

Not yet incorporated in Engelman's model is the effect of the cortical actin skeleton on the lateral organization and mobility of plasma membrane molecules. This aspect is discussed in the picket-and-fence model.[10] In addition to this more indirect steric hindrance, the direct interactions of the skeleton with proteins is a highly investigated aspect up to now. ${ }^{[3]}$

Figure 1.1 shows the structural organization of the plasma membrane, trying to include all mentioned aspects and models. 


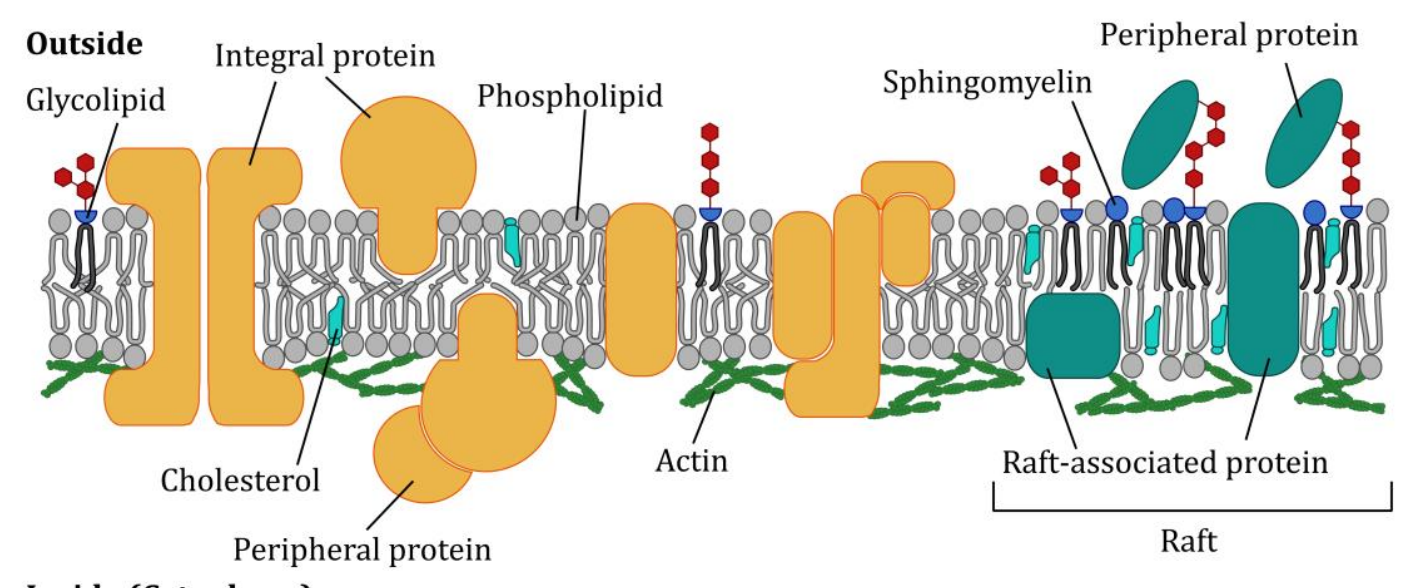

\section{Inside (Cytoplasm)}

Figure 1.1. Lateral organization of the plasma membrane. Proteins either are integrated in (integral) or attached to (peripheral) the bilayer formed by the different lipids and cholesterol. Clustering of sphingomyelin and cholesterol leads to 'lipid rafts' and a possible enrichment with certain proteins in these regions. The actin cytoskeleton influences organization and lateral diffusion of proteins and lipids. Image adapted from Lingwood and Simons.[11]

This variety of models illustrates that there is no general model sufficiently describing all aspects of the organization of the plasma membrane. As many functions of the plasma membrane and associated processes are influenced by this organization, one might have to refer to different models, depending on the process or function studied. Further, it has to be noted that every simplified artificial membrane system used for experimental investigations only represents certain aspects of the biological plasma membrane.

\subsection{Transport through bacterial membranes: bacterial porins}

The outer membrane of gram-negative bacteria, such as E. coli, Salmonella and Neisseria, exhibits like other biological membranes only a low permeability for polar and charged molecules. Thus, the bacterial cell needs channel proteins for the influx of nutrients and the efflux of waste products. This function is fulfilled by the so-called porins, which are water-filled pores spanning the whole membrane and enabling passive diffusion of hydrophilic solutes along existing electrochemical gradients.[12]

\subsubsection{Structure and function}

Originally, the term 'porin' was introduced to describe only nonspecific diffusion channels found in every gram-negative bacterium. These 'general' or 'nonspecific' porins act as molec- 
ular sieves, excluding molecules according to their size and charge. ${ }^{[13]}$ Additionally, some specific channels, such as LamB ('maltoporin') and PhoE ('phosphoporin') from E. coli are also defined as porins. ${ }^{[12]}$ In contrast to the general porins that present a linear relation between solute concentration and translocation rate, they contain specific binding sites for certain substrates and show a saturation of the translocation rate, similar to the Michaelis-Menten kinetics known from enzymes.[14] In the following, it will not be distinguished between the two types of porins, because they exhibit strong similarities in structure, properties and function. Besides, for some porins declared as nonspecific, like $0 \mathrm{mpF}$ from E. coli and Omp32 from Delftia acidovorans, specific substrate binding sites have been detected by now.[15,16]

Bacterial porins consist of 300 to 420 amino acids that form a 16- to 18-stranded antiparallel $\beta$-barrel. On the extracellular side, the $\beta$-strands are connected via long loops and on the periplasmic side by short loops and turns (Figure 1.2 B).[17] At about mid-height of the barrel, the pore is constricted by an internal loop (loop3) that is attached to the inside of the barrel wall and defines the constriction region. A defined charge distribution exists within this region and plays an important role in ion permeation and selectivity.[12,18,19] In most porins, negatively charged amino acid residues are found at the internal loop, whereas the opposed barrel wall contains mainly positively charged residues.[20]

The monomers of most porins associate to trimers (Figure $1.2 \mathrm{~A}$ ) by interaction of the hydrophilic parts of the barrel outside, leaving the hydrophilic regions accessible to detergents. This quaternary structure is further stabilized by loop2, which interacts with a neighboring monomer. The trimeric structure is extremely stable and can only be destroyed upon denaturation. $[17,20]$
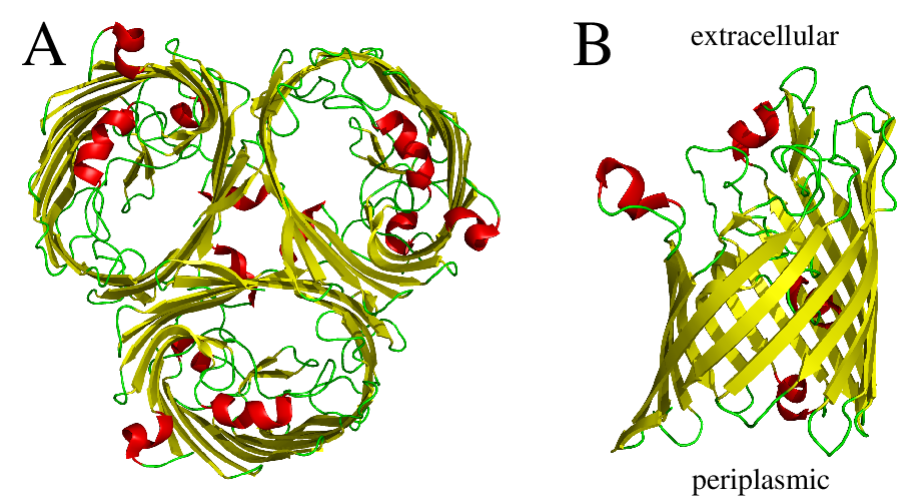

Figure 1.2. Crystal structure of $\mathrm{OmpF}$ from E. coli. Trimer shown from the top (A) and monomer as side view (B). The $\beta$-barrel is depicted in yellow, $\alpha$-helical structures in red, loops and turns in green. PDB-ID: 3K19.[21] 
Porins can switch between an open and a closed state (gating), which depends on the transmembrane potential.[12] Thus, they can be described as voltage-dependent channels. Although the exact mechanism of voltage gating is not known, conformational changes of the pore are often proposed to play a role. They can involve a motion of loop3 into the constriction zone,[22] rearrangements of the charges at the constriction region, ${ }^{[23,24]}$ movements of surface loops into the pore,[25] or be an intrinsic property of the pore itself, e.g., by large structural deformations.[26,27]

As porins are the main pathway for solutes into the bacterial cell, they play a key role in the permeation of antibiotics. Thus, their influence on antibiotic resistance of gram-negative bacteria is widely discussed.[12,28]

\subsubsection{Bacterial porins and antibiotic resistance}

Multidrug resistances are very frequently found in gram-negative bacteria. As they strongly limit the treatment options, they are largely responsible for complications and mortality in hospitals. ${ }^{[29,30]}$ In the review on antimicrobial resistance of the WHO published in May 2016, the number of deaths globally due to antimicrobial resistances is estimated as 700000 per year. This number is predicted to rise to 10 million in 2050.[31] Thus, understanding the mechanisms of antibiotic resistance in order to develop better-suited agents is a pressing topic of current research.

Three main bacterial strategies of antibiotic resistance are known. The expression of specific enzymes leads to a modification or even degradation of the drug, mutations or expression of molecules inhibiting target recognition impair the antibiotic activity and the influx of antibiotics through the outer membrane can be reduced or completely prevented.[32-34] The latter strategy can be supported by an increased expression of antibiotics efflux pumps.

In gram-negative bacteria, the influx of substances through the outer membrane is regulated by porins, e.g., OmpC and OmpF from E. coli and Salmonella, or PorB from Neisseria.[12,20,35] They are responsible for the permeation of antibiotics, such as the two important antibiotics groups of $\beta$-lactams and fluorochinolones.[36] Consequently, an impaired antibiotic uptake can be related to a modification of the involved porin. Such modifications can be the expression of a different porin type, a reduction of the porin expression or mutations influencing the functional pore properties (see Figure 1.3).[28] In Klebsiella pneumoniae, the level of susceptibility to $\beta$-lactams is 4-8 times lower if OmpK36 is expressed instead of OmpK35.[37] This is probably caused by the different sizes of the two pores: OmpK35 is the homologue of OmpF in E. coli and has a larger channel size than OmpK36, which belongs to the OmpC porin group. 
The high-level of chloramphenicol resistance of Salmonella typhi clinical isolates is related to the deficiency of the major porin OmpF.[38]

Mutations of porins related to altered antibiotic permeation often are located at the constriction region. A strong electrostatic field within this region defined by loop3 (see Chapter 1.2.1) influences the permeation pathways of ions and antibiotics. ${ }^{[12,20]}$ Consequently, mutations in the constriction region can alter the uptake of antibiotics by changing this electric field and thus induce resistances. Various mutations at the constriction zone of $\mathrm{OmpF}$ were found to change the susceptibility of $E$. coli for antibiotics ${ }^{[39]}$ and the high level of $\beta$-lactam resistance of Enterobacter aerogenes is accompanied by an amino acid substituted at the internal loop of Omp36.[40]

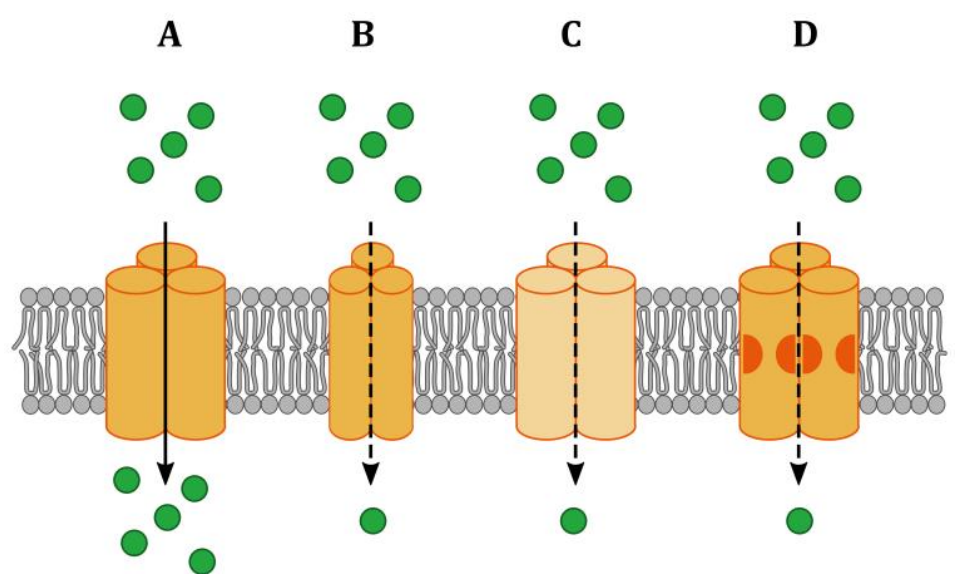

Figure 1.3. Alterations of the antibiotic's permeation related to porin modifications. The transport of antibiotics (green) across the bacterial outer membrane (grey) mediated by porins (orange) is shown. If the wild type porin is expressed at a normal level (A), the antibiotic's transport is not impaired. The expression of a porin with smaller channel size instead (B), the reduced expression of the wild type porin (C) and the expression of a porin mutant (D) can strongly reduce the antibiotic's permeation and cause resistances. Based on Pagès et al.[28]

The influence of such mutations on the permeation of antibiotics can be investigated using several methods. The liposome-swelling assay allows the qualitative analysis of the antibiotic's influx. Multilamellar liposomes filled with a polysaccharide increasing the optical density, e.g., dextran or stachyose, are prepared and mixed with an isosmotic solution containing the antibiotic. Only if there are porins incorporated into the liposomes enabling antibiotic's permeation, a water influx will balance the osmotic gradient and swell the liposomes until they rupture. The resulting decrease in optical density enables conclusions about the permeation rate. ${ }^{[28,41]} \mathrm{A}$ quantitative analysis of the interaction between antibiotic and pore can be realized using electrophysiological measurements (Chapter 3.6) and further insights into translocation pathways, binding sites and energy barriers are obtained via molecular dynamics simulations (see Chapter 3.7). 


\subsubsection{PorB from Neisseria meningitidis}

Neisseria is a gram-negative bacterium that has two pathogenic species, Neisseria gonorrhea and Neisseria meningitidis, causing gonorrhea and meningitidis specifically in humans.[42] Multidrug resistance is a major issue with Neisseria as well. The first cases of decreased penicillin susceptibility of Neisseria meningitidis were already reported in the 1970s and 1980s and by now Neisseria gonorrhea presents resistance against most common antibiotics, such as $\beta$-lactams, tetracyclines and quinolones. ${ }^{[43,44]}$

PorB is a major outer membrane protein (OMP) of Neisseria and shows $60 \%$ sequence similarity between Neisseria meningitidis (Nme) and Neisseria gonorrhea (Ngo).[35,45,46] The porin is reported to have a significant role during the disease progression, which is discussed in detail for PorB (Ngo).[47] PorB ( Ngo) is able to translocate from the outer membrane (OM) of Neisseria to the inner mitochondrion membrane (IMM) of the target cell using the translocase of the outer mitochondrial membrane (TOM; see Figure 1.4). The pores' open probability is increased by the non-covalent binding of ATP. Due to the uncontrolled flux of ions, the transmembrane potential of the IMM breaks down. The following loss of the cristae structure triggers the release of proapoptotic factores through the outer mitochondrion membrane (OMM), which results in cell death.

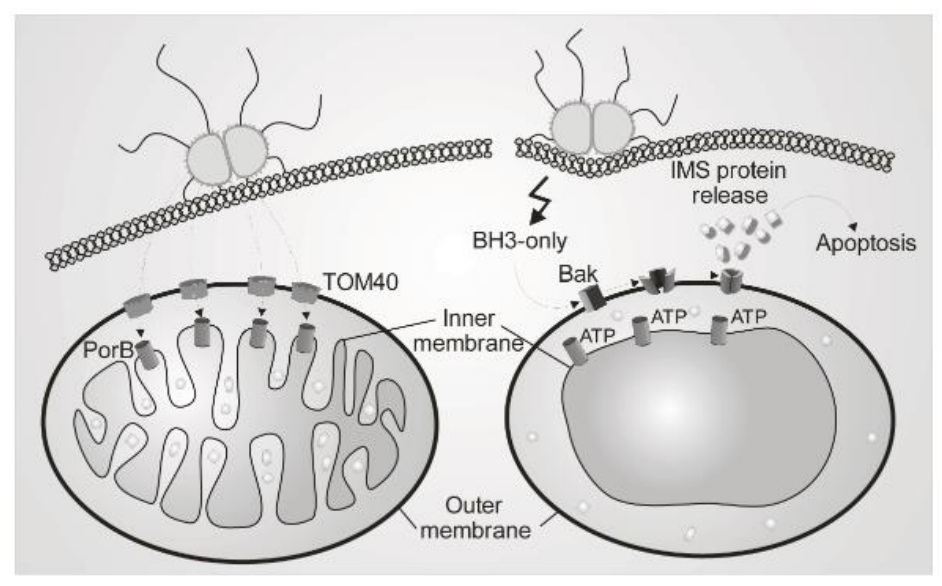

Figure 1.4. Proposed mechanism for the role of PorB $(\mathrm{Ngo})$ during disease progression. The porin inserts into the inner mitochondrial membrane (IMM) causing a breakdown of the transmembrane potential. The resulting loss of the cristae structure triggers the release of proapoptotic factors through the outer mitochondrial membrane (OMM), which eventually leads to cell death.[47]

PorB from Neisseria meningitidis consists of 341 amino acids forming a 16-stranded $\beta$-barrel and exhibits the typical trimeric porin structure described above. Loop3 constricts the pore to an area of 8 by $10 \AA$ at its narrowest point (see Figure 1.5 A).[35] The analysis of the electrostatic surface potential revealed an exceptional charge distribution between periplasmic and extracellular side not reported for other porins. The funnel approaching the constriction 
zone is mainly electronegative at the extracellular side (Figure $1.5 \mathrm{~B}$ ) and electropositive at the periplasmic side (Figure $1.5 \mathrm{C}$ ).
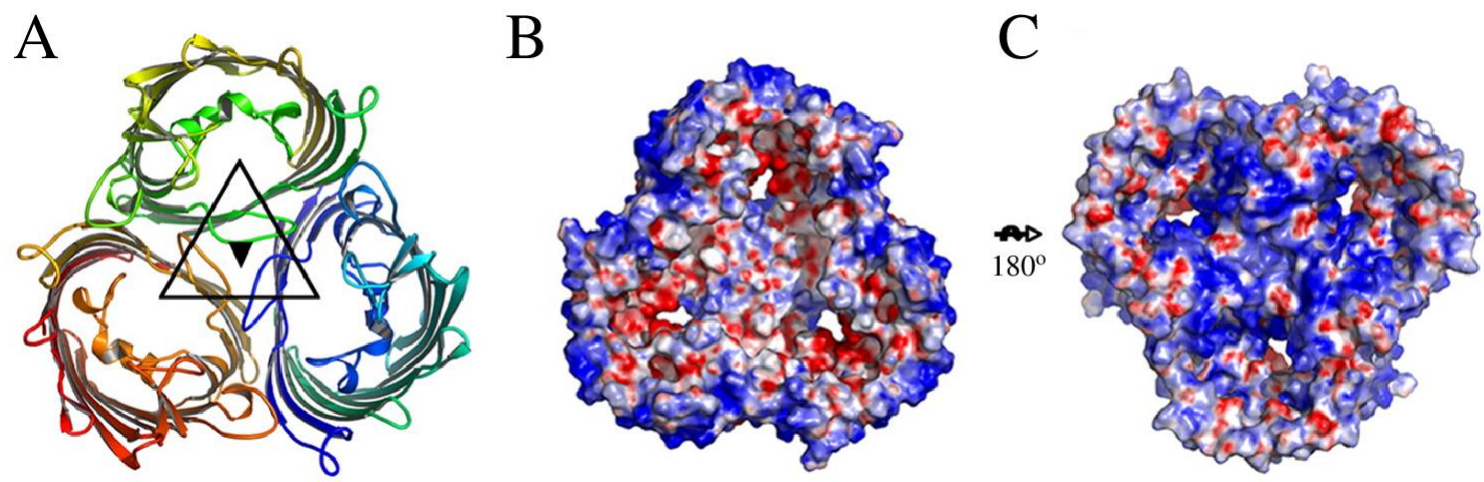

Figure 1.5. Trimeric structure of PorB (Nme). Cartoon representation from extracellular perspective (A). Electrostatic surface potential from extracellular (B) and periplasmic (C) perspective. Negative potential is depicted in red, positive potential in blue.[35]

Several binding sites and putative translocation pathways for different solutes were found in PorB (see Figure 1.6).[35] A position, where cations might be attracted was determined near the upper part of loop3 (orange circle). The residues of Asp117 and Glu202, as well as the backbone carbonyl of Gly122 are proposed to be involved. The analysis of the electrostatic surface potential leads to a putative translocation pathway for anions (violet shading) being consistent with the properties identified in other porins. This pathway includes four conserved arginine and lysine residues. A specific sugar binding site was determined for sucrose (light blue circle) and galactose (magenta circle) using a co-crystallization assay. It is surrounded by arginine and lysine residues and located within the positively charged funnel. As cation and anion pathways are overlapping neither with each other nor with the sugar-binding site, sugar transport and ion translocation probably occur independently. Based on cocrystallization, a binding site of the ATP analogue adenylylimidodiphosphate (AMP-PNP, black square), was identified as well, supporting the assumption that the role of PorB (Nme) in disease progression (see Figure 1.4) is similar to the mechanism proposed for PorB (Ngo). 


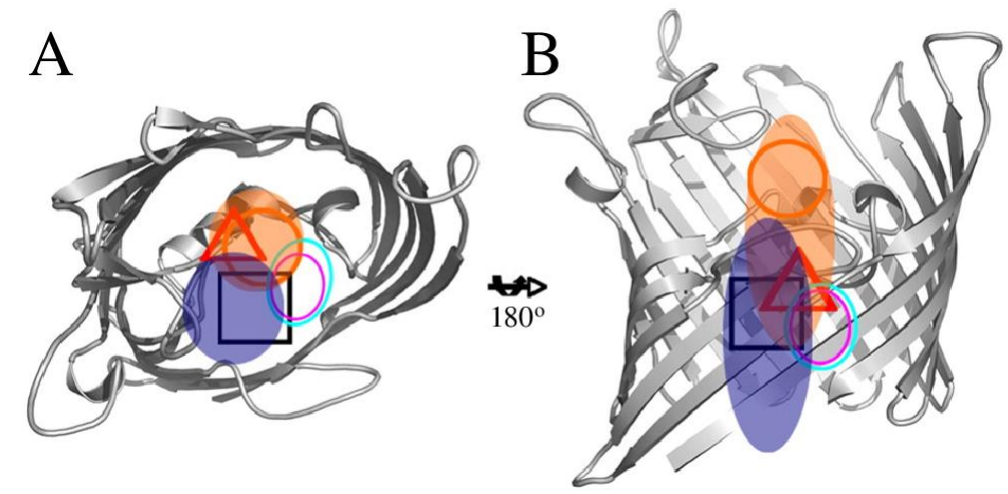

Figure 1.6. Binding sites and putative translocation pathways through PorB (Nme). PorB is shown from the extracellular side (A) and from the membrane plane (B). The cation binding site and translocation pathway (orange circle and shading) are independent of the anion pathway (violet shading) and the sugar-binding site (magenta: galactose, light blue: sucrose). A biding site for AMP-PNP (black square) was determined by co-crystallization and an antibiotic binding site (red triangle) is assumed near the cation biding site.[35]

A possible binding site for antibiotics (red triangle) is proposed to be below the cation-binding site, involving Asp104 and Gly103, which are located at loop3. This assumption is based on the observation that resistance of Neisseria gonorrhea to penicillin and tetracycline is related to mutations of PorB ( Ngo) at position 120 and 121,[48] which correspond to position 103 and 104 in PorB (Nme).

To further investigate the effect of mutations at positions 120 and 121 on the properties of PorB (Ngo), patch-clamp measurements of PorB wild type (wt) and several mutants were performed.[49] The main conductance state of the mutants turned out to be $30-40 \%$ lower than the one of the wild type. However, a difference in antibiotic permeation between PorB wt (Ngo) and the mutants was not detected during liposome swelling experiments. An electrophysiological investigation of PorB $(\mathrm{NgO})$ in the presence of antibiotics has not been performed.

PorB wt (Nme) has already been characterized using the patch-clamp technique,[50] but no electrophysiological investigations have been performed on mutated PorB (Nme) until now. Nevertheless, systematic studies on the effect of mutations at the internal loop on pore properties and antibiotic binding might be crucial to find out which role PorB (Nme) plays in the mechanism of antibiotic resistance of Neisseria meningitidis. 


\subsection{Investigation of antibiotic binding using the planar patch- clamp technique}

The planar patch-clamp technique (see Chapter 3.6) is used to investigate the electrophysiological properties of ion channels and porins. The application of a transmembrane voltage in the millivolt-range allows the time resolved measurement of currents through ion channels in the picoampere-range and enables determining channel characteristics like open channel conductance, open probability, ion selectivity and voltage dependence. ${ }^{[25,50]}$ Further, patchclamp recordings are a well-suited tool to study the interaction of substrates as sugars or antibiotics, with pores.[51-53]

A permeating antibiotic has a specific binging site within the pore. Once bound, it can partially or completely block the pore and impair the ion flux, which is visible as short interruptions of the open channel conductance (see Figure 1.7). The conductance level that is reached upon interaction with the antibiotic indicates whether one, two or all three monomers are blocked simultaneously.
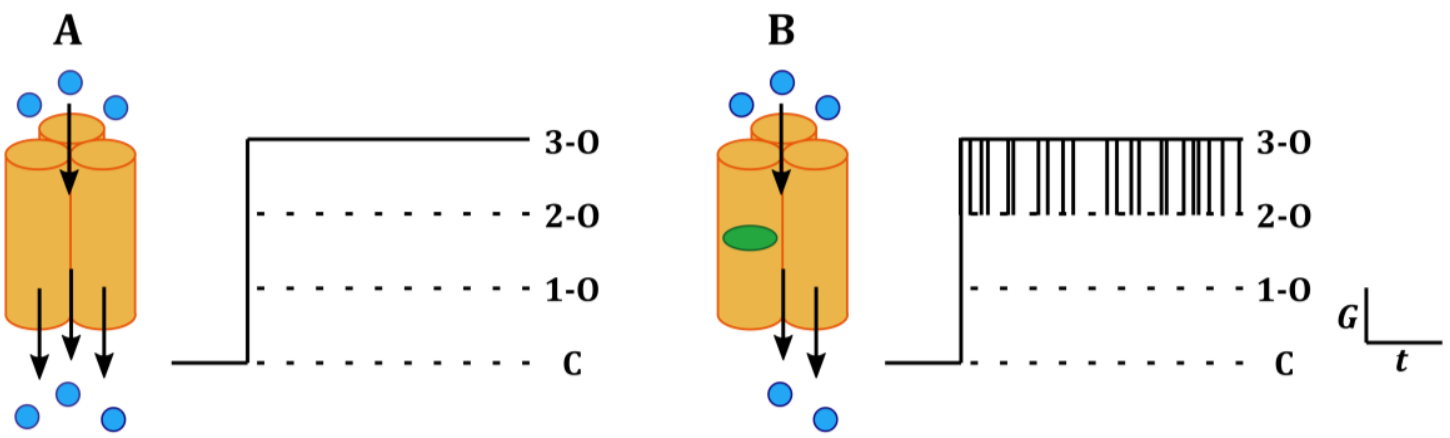

Figure 1.7. Model and schematic conductivity traces of a porin trimer. A fully open porin trimer (A) and a trimer with one monomer blocked by an antibiotic (B) are shown in comparison. Ions are depicted in blue and the antibiotic in green. In the conductivity traces the level of one (1-0), two (2-0) and three (3-0) open monomers and the fully closed trimer $(\mathrm{C})$ are indicated. Each blockage of the 3-0 state in B corresponds to the transient binding of an antibiotic molecule to the monomer.

The duration of these interruptions (blockage events) corresponds to the antibiotic's residence time, while the rate of the interruptions is the antibiotic's blockage frequency. The residence time yields information about the binding affinity between antibiotic and pore - longer residence time means a stronger binding - and the blockage frequencies allow the determination of the binding kinetics, i.e., $k_{\text {on }}$ and $k_{\text {off }}$ rates.[54] These characteristics then allow the direct comparison between different substrates or porins, e.g., the comparison between a wild type porin and its mutants. 
Blockage events can be analyzed by single event detection as done for channel insertion and gating events. As blockages occur much more frequent and show short durations, a manual evaluation is very time-consuming. Therefore, residence times and blockage frequencies are commonly determined by generating power spectra of the current fluctuations. The resulting power spectral densities are the frequency dependent amplitudes of the current noise from which the desired blockage parameters are obtained using a Lorentzian fit.[52,55] At bad signalto-noise ratios, however, this method is less accurate as the single event detection.

Addition of the antibiotic to either one side of the protein or the other can give first hints on the symmetry of the binding site, i.e., whether it is accessible from both sides and if binding affinity or kinetics differ between extracellular and periplasmic side of the pore. However, as only the binding of molecules and not the direction of release is detected, electrophysiology cannot provide clear evidence of a full translocation, but liposome swelling assays (see Chapter 1.2.2) can be used to confirm substrate influx through porins. Atomistic details about binding sites and permeation pathways can be obtained by molecular dynamics simulations, which are often used to complement and explain results from electrophysiology. ${ }^{[52,53,56]}$

\subsection{Interactions between bacterial porins and solutes}

Although porins are wide diffusion pores, they exhibit specific interactions with different types of solutes. These interactions can lead to binding or permeation of the solute, often but not necessarily binding is followed by a full translocation. In addition to monatomic ions and water, porins interact with larger solutes as ATP,[47] malate,[16] sugars,[51,57-59] and antibiotics. ${ }^{[52,53,55,56,60]}$ For many of them, specific binding sites, energy barriers and translocation pathways were detected by now.

Molecular dynamics (MD) simulations of the ion flux through OmpF of E. coli revealed two main interactions near the constriction region (CR). The $\mathrm{K}^{+}$ions were found close to the negative Glu117 residue, whereas the $\mathrm{Cl}^{-}$ions were near the cluster of positive arginine residues. ${ }^{[61]}$ For PorB of Neisseria meningitidis, completely separated pathways for $\mathrm{Cs}^{+}$and $\mathrm{Cl}$-ions were identified via MD simulations.[62]

Regarding the permeation of sugars, maltoporin (LamB) of E. coli is the most investigated porin. Single channel measurements showed a full translocation of maltodextrins consisting of three to seven glucose residues.[51] Only one sugar molecule interacts with a pore at a time, 
blocking the ion current completely. The energy barrier to enter the constriction zone from the extracellular vestibule is significantly lower than the one to leave it towards the periplasmic vestibule. ${ }^{[57]}$ Specific interactions involved in sugar translocation were detected performing MD simulations. ${ }^{[58,59]} \mathrm{A}$ "polar track" of amino acid residues facilitates sugar permeation via hydrogen bonds and hydrophobic interactions occur with a helical lane of aromatic residues. The sugar chains are described to slide "srew-like" along the aromatic lane while switching hygrogen bond partners. ${ }^{[59]}$

Due to the pressing subject of multidrug resistances, the interactions between porins and antibiotics are intensively investigated. Most studies involve OmpF from E. coli, as its structure is well known. Single channel measurements lead preferably to a blockage of the pore conductivity when zwitterionic antibiotics were involved. The zwitterionic $\beta$-lactams ampicillin and amoxicillin and the fluoroquinolone enrofloxacin were found to block the OmpF pores whereas the anionic piperacillin, azlocillin and carbenicillin did not.[52,53,56] Crystal structure analysis ${ }^{[15]}$ and MD simulations ${ }^{[52]}$ of $\mathrm{OmpF}$ in the presence of ampicillin and carbenicillin gave an explanation: While bound ampicillin is orientated perpendicular to the channel axis, carbenicillin binds parallel to the channel axis. Ampicillin is stabilized by hydrogen bonds and pi-stacking interactions between its phenyl ring and a hydrophobic pocket near several aromatic residues of OmpF. The lower number of hydrogen bonds between $\mathrm{OmpF}$ and the carboxylate group of carbenicillin leads to a higher flexibility of the antibiotic. In addition, swelling assays showed a lower permeation rate for the anionic antibiotic. ${ }^{[52]}$ Detailed insights into the translocation of antibiotics through OmpF were obtained by MD simulations. A specific binding site for ampicillin was found at the CR, accessible from both sides.[60] The antibiotic interacts with $\mathrm{OmpF}$ via hydrogen bonds and hydrophobic contacts, some hydrogen bonds are mediated by water molecules. During translocation, ampicillin changes its conformation, adapting a more compact form before entering the restricted area of the CR. A staircase of basic residues of OmpF interacts with the polar oxygen atoms of ampicillin to facilitate translocation. An exceptional situation was revealed for OmpF and enrofloxacin: Although a channel blockage was observed and a high binding affinity was determined, no full translocation of the antibiotic occurred. ${ }^{[53]}$ MD simulations revealed two binding sites at each side of the CR with a high energy barrier between them, making a passage of the antibiotic impossible.

Another important aspect during antibiotic translocation is the orientation of the solute's dipole.[63] This effect was first probed with water molecules permeating $\mathrm{OmpF}, \mathrm{OmpC}$ and two mutants of OmpC. The water dipole has to align according to the inner electric field of the 
pore. As this field changes between the different positions within the pore, the molecule has to reorient several times upon translocation. The adapted orientations vary between the different porins, because the inner electric field depends on the amino acid residues present. The same mechanism applies for antibiotics. They are pre-oriented by the electric field before entering the CR and have only to overcome a small energy barrier. However, they have to undergo a severe reorientation before leaving the CR. This mechanism can be expected to be different for each antibiotic due to the individual dipole.

The translocation of antibiotics and other solutes through bacterial porins depends on very specific interactions. Often single residues within the pore play a crucial role. Consequently, the exchange of single amino acids can dramatically influence the antibiotic translocation, thus lower the bacterium's susceptibility and possibly cause resistance. Therefore, understanding the influence of single point mutations on the interaction between pore and antibiotic might help revealing reasons for resistances and developing more effective antibiotics. 
1 Introduction 


\section{SCOPE OF THESIS}

The increasing multidrug resistances of gram-negative bacteria is a major cause of complications and mortality in hospitals, as they strongly limit the treatment options. Thus, finding and eliminating the causes of these resistances is a crucial task.

One bacterial strategy to increase antibiotic resistance is to prevent their influx into the cell. In gram-negative bacteria, the transport of antibiotics through the outer membrane is mediated by porins. They are $\beta$-barrels constricted by an internal loop that determines the constriction region (CR) often involved into the binding sites and permeation pathways of antibiotics found in porins. Hence, point mutations located at the CR can impair the uptake of antibiotics and induce resistance. To develop better-suited antibiotics or improve existing ones, an understanding of the interaction between porin and antibiotic and the influence of such mutations on this interaction is essential.

Neisseria is a gram-negative bacterium with two pathogenic species - Neisseria gonorrhea $(\mathrm{Ngo})$ and Neisseria meningitidis (Nme) - showing increasing resistance to most of the common antibiotics, such as $\beta$-lactams. Mutations in the major porin of Neisseria, PorB, are related to an increased level of resistance in $\mathrm{Ngo}$ and might probably cause resistance in Nme as well. Glycine-103 at the internal loop of PorB (Nme) was identified as possible key position.

The aim of this work is to investigate the influence of the mutations G103K and G103D in PorB (Nme) on the permeation of the $\beta$-lactam antibiotic ampicillin using the planar patchclamp technique supported by molecular dynamics (MD) simulations. The introduction of a positive (G->K) or a negative charge (G->D) together with a large residue can be expected to substantially alter the pore properties and thus the permeation of ampicillin.

First, the influence of the mutations on the electrophysiological properties of the pore, i.e., channel conductance and open probability, will be determined by comparing the wild type porin to the mutants. Based on these results, the interaction between ampicillin and PorB will be characterized investigating the blockage of the pore by the antibiotic. Comparing the blockage frequency and residence time of ampicillin in the wild type and the mutants allows drawing conclusions on the influence of the mutations on this interaction and thus on the antibiotic resistance of Nme. MD simulations will provide further insights concerning the permeation and binding site of ampicillin and support the interpretation of the electrophysiological data. 
2 Scope of thesis 


\section{Materials ANd Methods}

\subsection{Lipids}

\subsubsection{1,2-Diphytanoyl-sn-glycero-3-phosphocholine (DPhPC)}

The uncharged, artificial phospholipid 1,2-Diphytanoyl-sn-glycero-3-phosphocholine $\left(\mathrm{C}_{48} \mathrm{H}_{96} \mathrm{NO}_{8} \mathrm{P}, M=846.25 \mathrm{~g} / \mathrm{mol}\right.$, Figure 3.1 ) has a hydrophilic phosphocholine head group and two hydrophobic, saturated fatty acids (C16:0). Both fatty acids have methyl groups at position 3, 7, 11 and 15, which strongly influence the properties of the lipid. They lead to a high stability of the formed lipid bilayers which are very well suited for electrophysiological experiments. ${ }^{[64-66]}$ The thickness of a pure DPhPC bilayer including head groups was simulated as $60 \AA . .{ }^{[67]}$ Due to the size of the phosphocholine head group and the chain length, vesicles are readily formed.[68] The lipid aggregates show no transition from gel to liquid crystalline phase between temperatures of -120 and $+120^{\circ} \mathrm{C} .[69]$<smiles>CC(C)CCCC(C)CCCC(C)CCCC(C)CCCC(C)CC(=O)OC[C@H](COP(=O)([O-])OCC[N+](C)(C)C)OC(=O)CC(C)CCCC(C)C</smiles>

Figure 3.1. Structure of 1,2-Diphytanoyl-sn-glycero-3-phosphocholin (DPhPC).

\subsubsection{Cholesterol (Chol)}

Cholesterol $\left(\mathrm{C}_{27} \mathrm{H}_{46} \mathrm{O}, M=386.65 \mathrm{~g} / \mathrm{mol}\right.$, Figure 3.2) is a sterol and as such very important for mammalian cells. Due to its hydroxyl group, the molecule has an amphipathic character, is able to form hydrogen bonds to lipid molecules and is well-embedded in the lipid bilayer.[70,71] This can influence the mechanical and physical properties of the bilayer, such as stability and thickness.[66,72] The enhanced rigidity of lipid aggregates due to the presence of cholesterol[73] is advantageous for electrophysiological experiments, where stable membranes are required. 


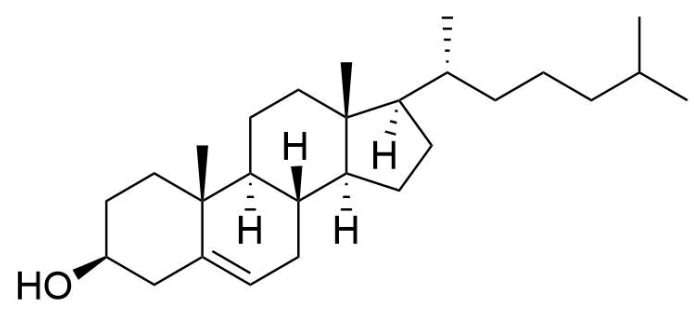

Figure 3.2. Structure of Cholesterol (Chol).

\subsection{PorB wild type and mutants}

In this project, PorB from Neisseria meningitidis was used. Figure 3.3 shows the sequence of 341 amino acids $(M=36.7 \mathrm{kDa})$ including secondary structure elements of PorB wild type. In addition to the wild type, the two mutants PorB G103K and G103D were investigated. They differ from the wild type by one amino acid at position 103 (located at the internal loop, see Chapter 1.2.3), where the glycine is substituted by a lysine (G103K) or an aspartic acid (G103D). Consequently, PorB G103K has a longer residue and an extra positive charge at the internal loop, whereas PorB G103D has a longer residue and an additional negative charge. PorB wt and the mutants were provided by Mikio Tanabe (Institute of Materials Structure Science, Structural Biology Research Center, Ibaraki, Japan). Expression and purification protocols are described elsewhere. ${ }^{[74,75,75]}$

\begin{tabular}{rrrrr}
10 & 20 & 30 & 40 & 50 \\
QVTLYGTIKA & GVEVSRVKDA & GTYKAQGGKS & KTATQIADFG & SKIGFKGQED \\
60 & 70 & 80 & 90 & 100 \\
LGNGMKAIWQ & LEQKASIAGT & NSGWGNRQSF & IGLKGGEGTV & RAGNLNTVLK \\
103 & 120 & 130 & 140 & 150 \\
\hline DSGDNVNAWE & SGSNTEDVLG & LGTIGRVESR & EISVRYDSPV & FAGFSGSVQY \\
160 & 170 & 180 & 190 & 200 \\
VPRDNANDVD & KYKHTKSSRE & SYHAGLKYEN & AGFFGQYAGS & FAKYADLNTD \\
210 & 220 & 230 & 240 & 250 \\
AERVAVNTAN & AHPVKDYQVH & RVVAGYDAND & LYVSVAGQYE & AAKNNEVGSI \\
260 & 270 & 280 & 290 & 300 \\
KGKKHEQTQV & AATAAYRFGN & VTPRVSYAHG & FKAKVNGVKD & ANYQYDQVIV \\
310 & 320 & 330 & 340 & \\
GADYDFSKRT & SALVSAGWLK & QGKGAGKVEQ & TASMVGLRHK & F
\end{tabular}

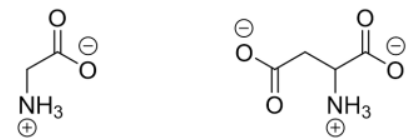

Glycine (G) Aspartic acid (D)

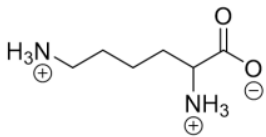

Lysine (K)

\section{Beta strand Betabridge bend turn helix}

Figure 3.3. Amino acid sequence of PorB wild type.[76] Secondary structure elements are marked in different colors. Exchanging the glycine at position 103 by lysine or aspartic acid leads to the mutants G103K and G103D, respectively. In G103K, an additional positive charge is present, whereas G103D is characterized by an additional negative charge. 


\subsection{Ampicillin}

Ampicillin (D-(-)- $\alpha$-Aminobenzylpenicillin, $\mathrm{C}_{16} \mathrm{H}_{19} \mathrm{~N}_{3} \mathrm{O}_{4} \mathrm{~S} \quad M=349.41 \mathrm{~g} / \mathrm{mol}$, Figure 3.4) is a zwitterionic antibiotic belonging to the group of $\beta$-lactams. The isoelectric point of ampicillin is at about $\mathrm{pH} 5$ and its $\mathrm{pK}_{\mathrm{s}}$ values are $\mathrm{pK}_{1}=2.66$ and $\mathrm{pK}_{2}=7.24 .{ }^{[77]}$ The proportion of the anionic and zwitterionic form of ampicillin at different $\mathrm{pH}$ values can be estimated from a graph of percent species vs pH.[78] At pH 6, $94 \%$ of the molecules exist in the zwitterionic form, whereas at pH $7.560 \%$ are in the anionic form. The antibiotic effect of $\beta$-lactams is based on the inhibition of enzymes essential during the bacterial cell wall synthesis. ${ }^{[79]}$

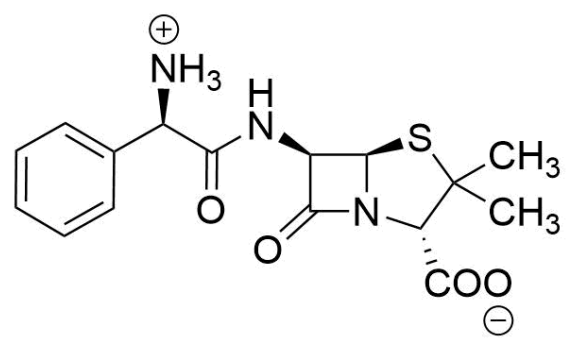

Figure 3.4. Structure of the $\beta$-lactam antibiotic ampicillin.

\subsection{No-SDS PAGE}

The sodium dodecyl sulfate - polyacrylamide gel electrophoresis (SDS-PAGE) described 1970 by Laemmli is used to characterize proteins by separating them according to their electrophoretic mobility. This is enabled by non-covalent binding of the anionic detergent SDS to the proteins, causing the denaturation of the proteins and resulting in an overall negative charge relative to their molecular weight. ${ }^{[80]}$ The polyacrylamide gel consists of a stacking gel where the proteins are accumulated and a resolving gel where they are separated. As SDS destroys the quaternary trimer structure of PorB, it was neither added to the gels nor to the sample buffer like in a standard SDS-PAGE, but was only present in the running buffer to ensure the overall negative charge while maintaining the trimeric structure. ${ }^{[81,82]}$

In this work, the Mini-PROTEAN@ Multi-Casting Chamber (Bio-Rad Laboratories, Inc., Hercules, CA, USA) was used. The gel was formed by polymerization of acrylamide and bisacrylamide using the radical starter ammonium peroxydisulfate (APS) and the stabilizer $\mathrm{N}, \mathrm{N}, \mathrm{N}^{\prime}, \mathrm{N}^{\prime}-$ tetramethylethane-1,2-diamine (TEMED). The compositions of all solutions and buffers used are summarized in Table 3.1. First, the solution for the resolving gel was prepared, filled into 
the gel chamber and overlayed with isopropanol. After polymerization for 45-60 min, the isopropanol was removed and the solution for the stacking gel was added on top of the resolving gel. A comb was inserted into the stacking gel to form the sample wells. After at least $60 \mathrm{~min}$, the polymerization was finished and the comb could be removed. Subsequently, the gel was inserted into the electrophoresis chamber. $10 \mu \mathrm{L}$ of each protein sample were mixed with $10 \mu \mathrm{L}$ sample buffer and heated at $45^{\circ} \mathrm{C}$ or $95^{\circ} \mathrm{C}$, respectively, using a thermomixer $(5 \mathrm{~min}$, $300 \mathrm{rpm}$ ). The samples and a molecular marker (Thermo Scientific ${ }^{\mathrm{TM}}$ PageRuler $^{\mathrm{TM}}$ Plus prestained protein ladder $10-250 \mathrm{kDa}$ ) were pipetted into the sample wells and the electrophoresis chamber was filled with running buffer. Electrophoresis was started by applying $200 \mathrm{~V}$ and finished after about 30-40 s. Bromophenol blue was removed by heating the gel several times for 30-45 s in water using a microwave followed by 1 min of agitation using a shaking board. To stain the gel, it was placed into Water-Coomassie solution and heated for $30 \mathrm{~s}$ in the microwave, followed by $5 \mathrm{~min}$ agitation using the shaking board. To remove excess Coomassie staining, the gel was repeatedly agitated in water using the shaking board until the protein bands were clearly visible.

Table 3.1. Compositions of solutions and buffers used for the gel electrophoresis.

Resolving gel

Stacking gel

Sample buffer

Running buffer staining solution
$1.68 \mathrm{~mL} \mathrm{30 \%} \mathrm{acrylamide-bisacrylamide} \mathrm{(29:1)}$

$1.04 \mathrm{~mL} 1.5 \mathrm{M}$ TRIS/HCl pH 8.8

$1.28 \mathrm{~mL} \mathrm{H}_{2} \mathrm{O}$

$2 \mu \mathrm{L}$ TEMED

$40 \mu \mathrm{L} 10 \%(w / v)$ APS

$0.34 \mathrm{~mL} 30 \%$ acrylamide-bisacrylamide (29:1)

$0.25 \mathrm{~mL} 1 \mathrm{M}$ TRIS/HCl pH 6.8

$1.41 \mathrm{~mL} \mathrm{H}_{2} \mathrm{O}$

$2 \mu \mathrm{L}$ TEMED

$20 \mu \mathrm{L} 10 \%(w / v)$ APS

175 mM TRIS/HCl pH 6.8

$0.1 \mathrm{M} \mathrm{DTT}$

$20 \%(w / v)$ glycerol

$0.02 \%(w / v) 2 \mu$ g Bromophenol blue

$25 \mathrm{mM}$ Tris/HCl

$192 \mathrm{mM}$ glycine

$0.1 \%(w / v)$ SDS

pH 8.3

$0.08 \%(w / v)$ Coomassie-Brilliant-Blue G-250

$3 \%(v / v) \mathrm{HCl}$ 


\subsection{Electroformation}

The giant unilamellar vesicles (GUVs, $d=10-100 \mu \mathrm{m}$ ) used for the measurements at the Porta-Patch (Chapter 3.6.3.1) were prepared using electroformation. This method under low salt conditions is based on the rehydration of dried lipid films supported by an alternating voltage.[83] Thus, the lipid films have to be formed on a conducting surface. Formation rate and size of the GUVs are influenced by the rehydration solution, the applied voltage and the frequency of the alternating field. ${ }^{[84]}$ Sugar solutions have a stabilizing effect.[85]

In this work, glass slides coated with Indium-Tin-Oxide (ITO) were used. Two ITOs separated by Teflon spacers were combined to an electroformation chamber hold together by metal clips. A Teflon ring sealed the rehydration solution and copper stripes served as electrodes. First, the desired lipids $(0.2 \mathrm{mg})$ dissolved in chloroform were added to the two ITO slides. After removal of the solvent under vacuum, the electroformation chamber was put together and filled with $1 \mathrm{M}$ sucrose solution. An alternating voltage of $3 \mathrm{~V}_{\mathrm{pp}}$ and $5 \mathrm{~Hz}$ was applied for $2 \mathrm{~h}$ using the frequency generator Agilent 3220A (Agilent Technologies, Santa Clara, CA, USA). Finally, the solution containing the formed GUVs was harvested, discarding the first $500 \mu \mathrm{L}$, as most of the GUVs are found in the lower part of the chamber.

\subsection{The planar patch-clamp technique}

The first experiments to study the electric properties of a cell membrane were performed by Cole and Marmont who developed the voltage-clamp technique at the same time.[86,87] They discovered that a transmembrane potential can be applied using two electrodes. Based on these findings, Neher and Sakman developed the patch-clamp technique and published the first recordings in 1976. ${ }^{[88]}$ Placing micropipettes containing electrodes onto a cell allowed the first single-channel recordings. In 1980, they realized that gentle suction strongly improved the seal between cell and pipette, leading to resistances in the gigaohm range (gigaseal) and significantly lowering the background noise. ${ }^{[89]}$ At the same time as Horn and Patlak, ${ }^{[90]}$ Hamill and Sakman [91] discovered that it was possible to remove small patches from a cell by applying suction and simultaneously withdrawing the pipette. The so-called patch mode allowes investigating the effect of drugs or different ion concentrations by exchanging the bath solution. ${ }^{[89,91]}$ 
Performing patch-clamp experiments using micropipettes is laborious work requiring a lot of experience and the obtained membrane patches are often not very stable. Thus, the development of the planar patch-clamp technique was a significant improvement. In this technique, large parts of the membrane are stabilized by a planar substrate, while only a small part is spanning an aperture within this substrate. Planar patch-clamp can be used to investigate both, cells or artificial membranes. A further advantage is the bilateral accessibility of the membrane patch allowing the individual exchange of the bath solutions.

\subsubsection{Theoretical background and electronic setup}

Membranes in aqueous solution act at the same time as insulator and capacitor. Perfectly sealed membranes are almost completely insulating with resistances of several giga-ohms. This gigaseal impairs free diffusion of charged particles through the bilayer and is therefore crucial to obtain the necessary signal-to-noise ratio.[92] Typical transmembrane potentials of animal cells are usually found between +30 and $+90 \mathrm{mV} .{ }^{\left[{ }^{[2]}\right]}$ Applying transmembrane potentials in the millivolt range enables measuring even smallest currents in the nano- to picoampere range. During a patch-clamp experiment, applying a transmembrane potential $U_{\mathrm{m}}$ and continuously measuring the resulting current $I$ gives information about the embedded channel proteins.

Biological membranes are relatively thin, in the range of 7-10 $\mathrm{nm} .{ }^{[93,94]}$ Typical resting potentials therefore induce considerably large electrical fields. For example, a resting potential of approximately $+100 \mathrm{mV}$ across a membrane of $10 \mathrm{~nm}$ thickness results in an electric field in the range of $10^{5} \mathrm{~V} / \mathrm{cm} .{ }^{[92]}$ Values like this normally cause an irreversible breakdown of an insulator, because its atoms become ionized. In an electrophysiological experiment, these fields are only measured indirectly. Voltage-sensitive ion channels react to changes in the electric field by opening and closing, which is detected by changes of the current signal.

Capacitance $C$ (measured in Farads, F) describes the charge $Q$ stored in a capacitor at a voltage difference $\Delta U$ existing across its two ends (see Equation 3.1).

$$
C=\frac{Q}{\Delta U}
$$

As parallel conducting plates separated by a thin insulating layer are the strongest capacitors, the symbol of two parallel lines was established. Thereby, the capacitance increases with the area $A$ of these plates and decreases with their distance $d$ : 


$$
C=\varepsilon_{0} \varepsilon \frac{A}{d}
$$

$\varepsilon$ is the permittivity of the medium between the two plates and $\varepsilon_{0}$ the vacuum permittivity. Consequently, membranes are very good capacitors due to their low thickness. The polar head groups thereby represent the conducting plates separated by the insulating lipophilic hydrocarbon chains. The capacitance of a membrane is indicated per unit area and is for most cell membranes about $1 \mu \mathrm{F} / \mathrm{cm}^{2}{ }^{2}{ }^{[95,96]}$

Capacitors store charge solely in the presence of a voltage difference across the capacitor. The current $I$ flowing through the capacitance thus depends on the voltage change with time $t$ (see Equation 3.3).

$$
I=C \frac{\Delta U}{\Delta t}
$$

The influence of the membrane capacitance on the current flow through embedded ion channels can be neglected in case of a constant transmembrane voltage. Any alteration of the transmembrane voltage results in a change of the charge stored in the membrane capacitance. An applied current, e.g., by an electrode, first charges the membrane capacitance before changing the membrane voltage (see Figure $3.5 \mathrm{C}$ and $\mathrm{D}$ ). This behavior is best described by an equivalent circuit consisting of a resistor $R$ in parallel with a capacitance $C$ (see Figure 3.5 B).

A

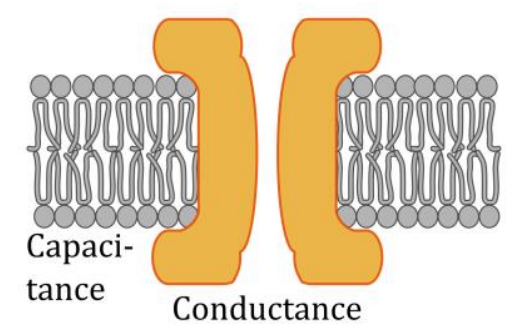

B

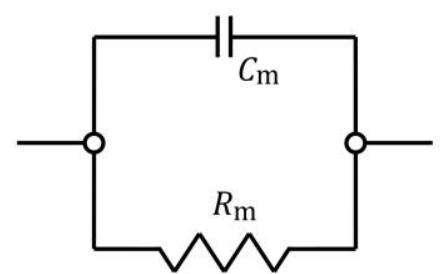

C

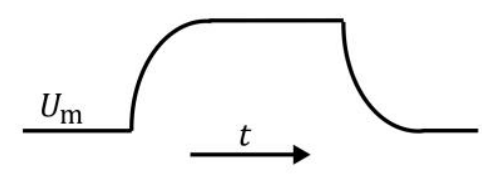

D

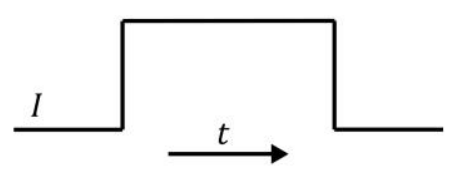

Figure 3.5. Electrical properties of a lipid membrane. Ion channel containing membrane $(A)$ and the corresponding equivalent circuit (B). Voltage response (C) of the membrane upon an applied current pulse (D). The capacitor is charged before the membrane voltage is changed.

The time dependent membrane voltage $U_{\mathrm{m}}(\mathrm{t})$ reaches an equilibrium value $U_{\mathrm{inf}}$ after an exponential increase or decrease, respectively (compare Figure $3.5 \mathrm{C}$ ). This behavior is expressed 
by Equation 3.4. $U_{\text {inf }}$ is defined by Ohm's law (Equation 3.5 ) and thus only depends on the current $I$ and the membrane resistance $R_{\mathrm{m}}$, not on the capacitance.

$$
\begin{gathered}
U_{\mathrm{m}}(t)=U_{\mathrm{inf}} \cdot\left(1-e^{-t \tau^{-1}}\right) \\
U_{\mathrm{inf}}=I \cdot R_{\mathrm{m}}
\end{gathered}
$$

The charging time constant $\tau$ describes the time necessary to reach the membrane voltage. It is defined by the membrane capacitance $C_{\mathrm{m}}$ and the membrane resistance $R_{\mathrm{m}}$ :

$$
\tau=R_{\mathrm{m}} \cdot C_{\mathrm{m}}
$$

Electrophysiological experiments can be conducted as current clamp or voltage-clamp experiments. The former is performed by applying a certain current while measuring the membrane voltage. For the latter, the membrane voltage $U_{\mathrm{m}}$ is set and the compensatory current $\Delta I$ necessary to uphold this voltage is measured. In contrast to the current clamp experiment, the voltage-clamp experiment does not mimic a biological process. However, it has certain advantages. Capacitive currents disturbing the measurement only occur during the charging time (see Figure 3.6), the flowing currents are proportional solely to the membrane conductance and thus to the number of inserted channels and it enables the determination of the key channel characteristics because channel gating is determined only by the transmembrane voltage.
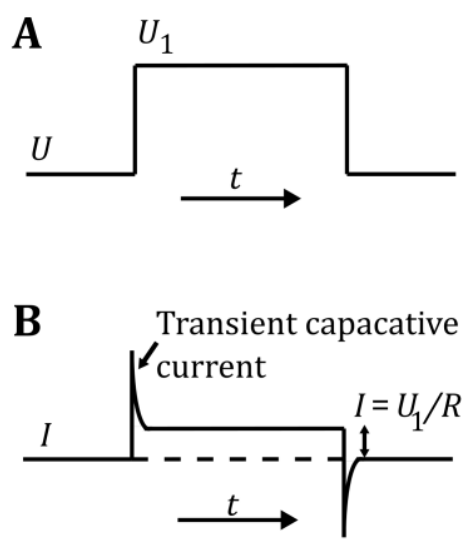

Figure 3.6. The voltage-clamp experiment. Time course of the applied voltage (A) and the current response (B). Each change of the voltage leads to a transient capacitive current, but apart from that, no capacitive currents influence the measurement.

In the presence of an open ion channel, the membrane resistance is no longer constant over time. Due to the passing ions, the actual resistance $R_{\mathrm{m}, \mathrm{c}}$ is lower than $R_{\mathrm{m}}$. Equation 3.7 describes the open channel conductance $G_{\mathrm{c}}$, with $G_{\mathrm{m}}$ being the membrane conductance and $G_{\mathrm{m}, \mathrm{c}}$ being the membrane conductance in the presence of the open channel.

$$
G_{c}=\frac{1}{R_{\mathrm{m}, \mathrm{c}}}-\frac{1}{R_{\mathrm{m}}}=G_{\mathrm{m}, \mathrm{c}}-G_{\mathrm{m}}
$$


Resistance and therewith conductance of the membrane can be considered as invariant over the time course of the measurement. Thus, the channel conductance is obtained by the change of the compensatory current $\Delta I$ and the applied membrane voltage $U_{\mathrm{m}}$ :

$$
G_{\mathrm{c}}=\frac{\Delta I}{U_{\mathrm{m}}}
$$

In the case of several open channels in one membrane, the overall conductance is the sum of all individual channels' conductance.

The electronic setup of a patch-clamp experiment consists of two separated electrical circuits (Figure 3.7). A signal generator transfers the desired transmembrane voltage to the main amplifier. The membrane is charged by a current pulse and the actual existing transmembrane voltage $U_{\mathrm{m}}$ is continuously detected and transmitted from the preamplifier to the main amplifier. When a channel opens, the flowing current reduces $U_{\mathrm{m}}$. This change is detected and compensated via a capacitive feedback loop by applying a compensatory current $\Delta I$. The feedback loop in the main amplifier uses the gains $\alpha$ and $\beta$ to amplify the signal and transmits it as a voltage signal. After filtering by the main amplifier, the signal is digitized by an A/Dconverter and recorded by a computer. Preamplifier and measuring chamber are shielded by a faraday cage and all equipment is grounded to avoid damage of the equipment and disturbances of the measurements.

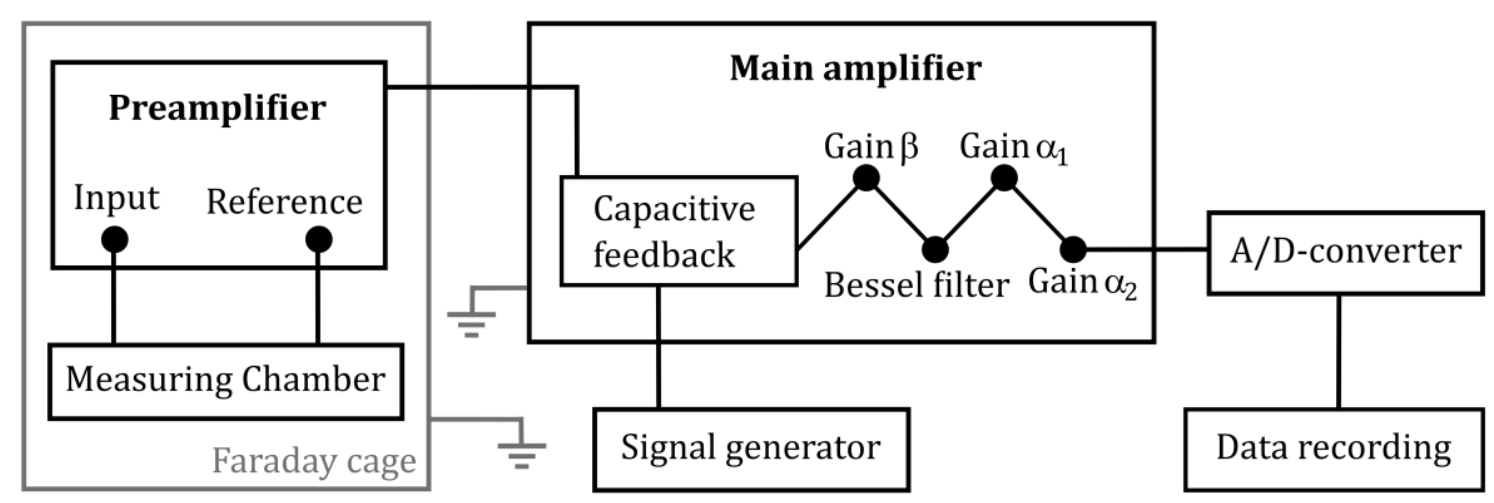

Figure 3.7. Electronic setup of a patch-clamp experiment. The preamplifier is connected to the measuring chamber by an input and reference electrode. It transmits the signal to the main amplifier that contains the capacitive feedback. The filtered signal is digitized by the A/D-converter and recorded by the computer.

The number of recorded data points is set by the sampling rate and the signal can be filtered with a chosen filter frequency to obtain the best signal-to-noise ratio for each experiment. It has to be noticed, that a low pass filter reduces the time resolution. Equation 3.9[97] shows how the cutoff frequency $f_{\mathrm{c}}$ (in hertz) of a low pass filter limits its rise time $t_{\mathrm{R}}$ :

$$
t_{\mathrm{R}}=\frac{0.35}{f_{\mathrm{c}}}
$$


At a chosen filter frequency of e.g., $1 \mathrm{kHz}$, the rise time is about $350 \mu \mathrm{s}$. This means that a channel gating at a shorter time scale as $350 \mu$ s will not be detected during the measurement.

\subsubsection{Membrane systems for planar patch-clamp}

\subsubsection{Solvent-free membranes}

Solvent-free membranes are formed by spreading of giant unilamellar vesicles (GUVs) over a small aperture on a planar substrate (see Figure 3.8). ${ }^{[98,99]}$ The protein of interest is incorporated by either adding a stock solution to a stable bilayer or spreading of protein-containing GUVs. The resulting membrane system is surrounded by a buffer solution, so that no organic solvent is present in the bilayer.

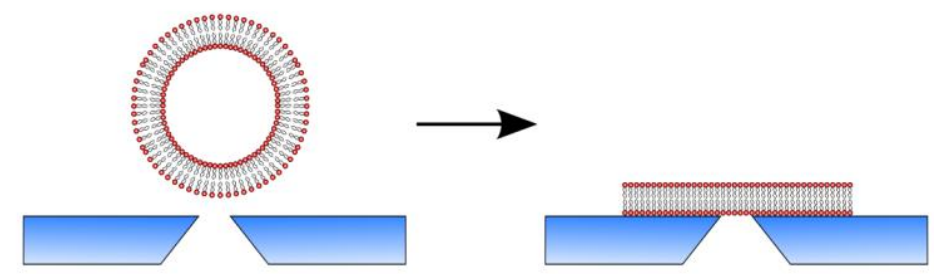

Figure 3.8. Preparation of a solvent-free membrane. A giant unilamellar vesicle is added to a planar glass substrate. The spreading of the GUV over a small aperture leads to a free-standing membrane without organic solvent present.

The advantage of this model system is the absence of organic solvents that might influence the properties of the inserted protein. The obtained membranes are close to the biological cell membrane.

However, solvent-free membranes are sensitive to some detergents[100] that are often present in protein stock solutions to ensure the stabilization of the protein structure.

\subsubsection{Black lipid membranes}

Black lipid membranes (BLMs) are widely used to study the electrophysiological properties of channel proteins.[101-103] They are formed by adding lipids dissolved in organic solvents to an aperture in a Teflon foil. The removal of the solvent leads to the formation of a bilayer (see Figure 3.9).[104] Membranes formed this way are no "patches" in the proper sense. Nevertheless, electrophysiological measurements conducted on BLMs will be termed as patch-clamp experiments. Protein can be added as a stock solution or by fusion of protein-containing liposomes. 


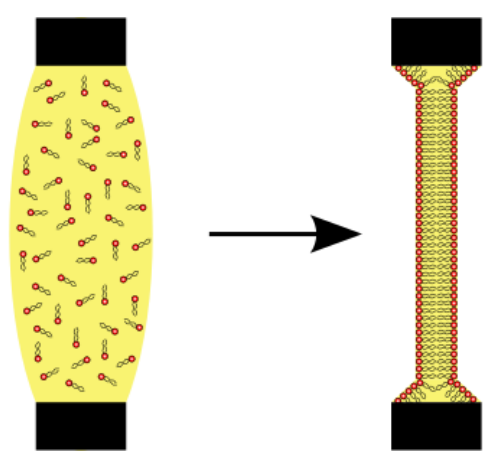

Figure 3.9. Preparation of a black lipid membrane (BLM). A mixture of lipids and organic solvent is added to an aperture in a Teflon foil. Removal of the solvent leads to a bilayer (painting).

With this model system, some organic solvent always remains in the bilayer. A positive consequence is the lowered membrane tension[66] that leads to very stable membranes not influenced by detergents. On the other hand, this organic solvent can have an effect on properties and function of the inserted proteins.[105,106]

\subsubsection{Experimental setups and realization}

Three different setups were applied to perform the patch-clamp experiments on PorB. The Port-a-Patch uses solvent-free membranes, whereas the self-constructed BLM setup and the Orbit 16 require solvent-stabilized bilayer. Due to the different advantages and disadvantages of the two membrane systems (see Chapter 3.6.2), they were used in parallel during the first part of the project (Chapter 4.2) to ensure a profound characterization of PorB. In the second part, only the solvent-stabilized bilayers turned out to be suited (compare Chapter 4.3).

\subsubsection{Solvent-free membranes}

The investigations on solvent-free membranes were performed using the Port-a-Patch (Nanion Technologies, München, Germany), together with planar borosilicate chips (NPC-1 Chips, Nanion Technologies, München, Germany, Figure 3.10). The Port-a-Patch (Figure 3.11 B) consists of a faraday cage, contacts to mount the electrodes and a thread where the borosilicate chip can be easily screwed on to seals the chamber underneath, to which a vacuum can be applied using the associated pump. Membranes were formed by spreading GUVs to the planar glass surface applying a small vacuum (see Figure 3.8).[98,99] The small aperture diameter about $1 \mu \mathrm{m}{ }^{[98,99]}$ leads to a small area of the free-standing membrane and thus facilitates the measurements of single-channels. 


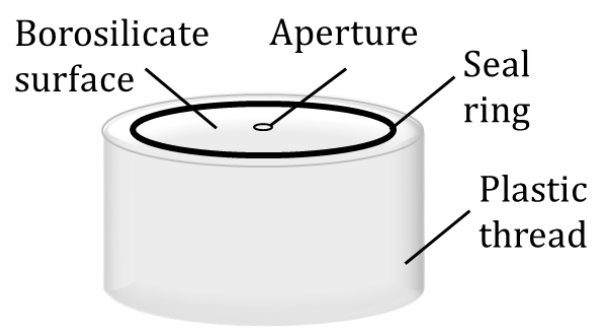

Figure 3.10. Borosilicate substrate used for the measurements at the Port-a-Patch. The aperture in the borosilicate surface has a diameter of several micrometers and the plastic thread is used to attach the chip to the device.

As main amplifier the Axopatch 200B (Axon Instruments, Union City, CA, USA), including a four-pole low pass Bessel filter, and as A/D converter the Digidata 1322A (Axon Instruments, Union City, CA, USA) were used. Clampex version 10.4.0.36 from the pClamp 10.4 software package (Molecular Devices, Sunnyvale, CA, USA) was used to conduct the measurements and the program PatchControl (Nanion Technologies, München, Germany) to control the pump. The schematic setup of the Port-a-Patch experiment is shown in Figure 3.11 A.

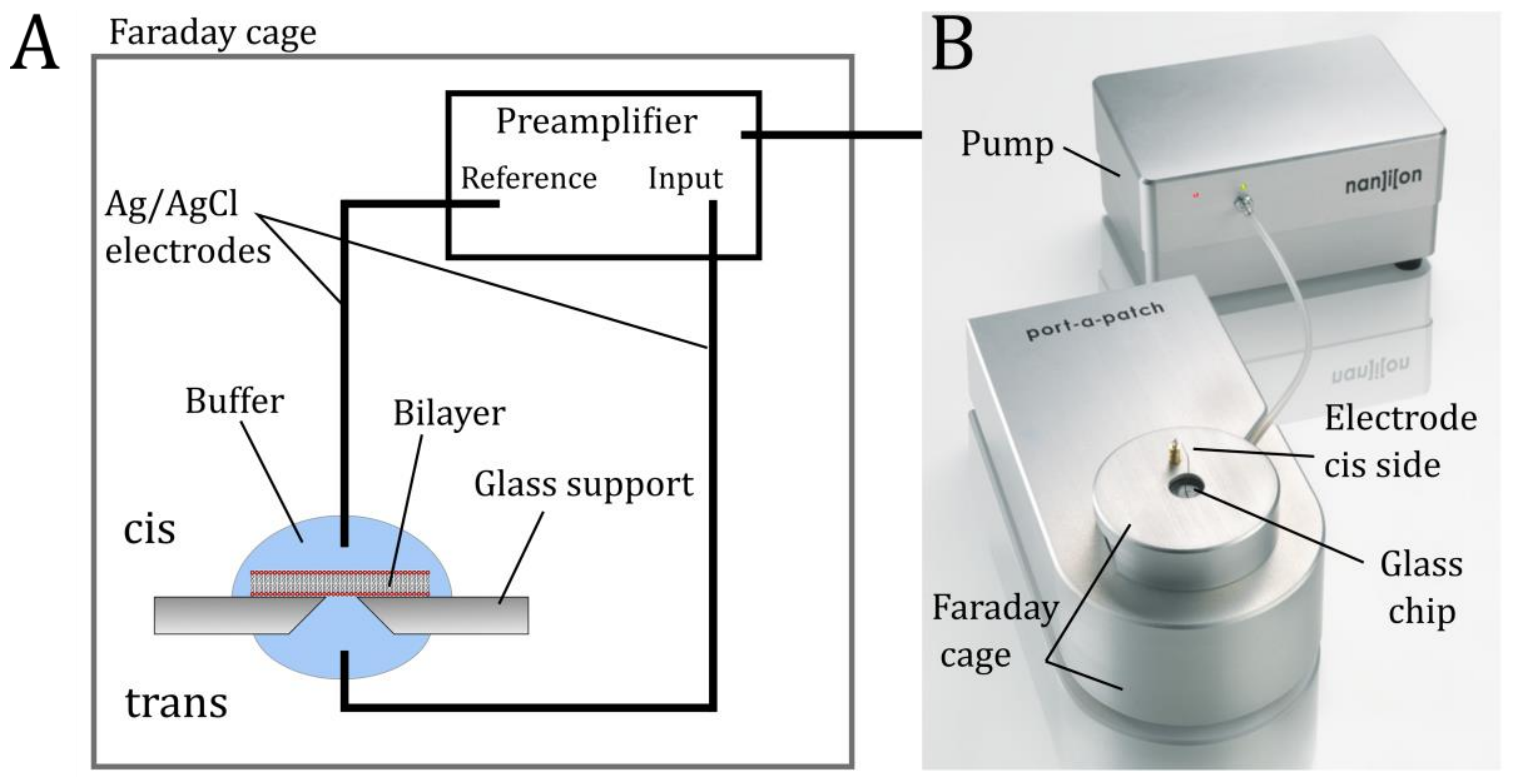

Figure 3.11. Patch-clamp experiment using the Port-a-Patch. Scheme of the experimental setup (A). A drop of buffer is placed on each side of the borosilicate chip and the bilayer is formed at the cis side (reference electrode). Image of the Port-a-Patch (Nanion Technologies, München, Germany), including the associated pump (B).[107]

\section{Realization}

Beforehand, the silver/silver chloride electrodes were prepared. After removing contaminations using sand paper, the electrodes were stored in $12 \%$ sodium hypochlorite solution for $20 \mathrm{~min}$. After preparation, as well as after the experiment, they were rinsed with ethanol and water. Subsequently, the electrodes were connected to the gold contacts. $6 \mu \mathrm{L}$ measuring buffer (1 M KCl, $10 \mathrm{mM}$ HEPES, pH 7.5) were added to each side of the aperture in the glass 
chip, which was then screwed on the Port-a-Patch. Finally, the top of the faraday cage was mounted.

Using the patch mode of the amplifier and the program membrane test, it was checked for a measurable electric current, meaning both electrodes were in contact with the buffer and the aperture freely permeable. If no current was measured, the electrodes were readjusted and the aperture freed from dust or air bubbles by applying 10 mbar pressure using the pump. Next, $4 \mu \mathrm{L}$ GUV suspension in sucrose (see Chapter 3.5) were added to the upper side of the glass chip (cis side, reference electrode) and immediately negative pressure (20-40 mbar) was applied to suck one GUV to the aperture and induce spreading. Successful membrane formation was visible in membrane test by a resistance of several giga-ohms (gigaseal). After membrane formation, the cis side was gently rinsed three times with $40 \mu \mathrm{L}$ buffer, followed by addition of $40 \mu \mathrm{L}$ buffer (overall volume of $50 \mu \mathrm{L}$ ) and the vacuum was turned off. Changing from membrane test to the desired measuring program, the membrane stability was tested by applying $+40 \mathrm{mV}$ for several minutes. Then, PorB (2.2-17.6 $\mu \mathrm{M}$ in $200 \mathrm{mM} \mathrm{NaCl}, 20$ mM Tris, $0.1 \%(w / w)$ LDAO, pH 7.5) was added in 0.5-1 $\mu \mathrm{L}$ volumes followed by 5 min incubation time until successful insertion. Current traces ( $60 \mathrm{~s}$ each) of PorB wt and G103K were recorded continuously at $+40 \mathrm{mV}, 10 \mathrm{kHz}$ sampling rate and $1 \mathrm{kHz}$ filter frequency at $20^{\circ} \mathrm{C}$. For PorB G103D the sampling rate was changed to $50 \mathrm{kHz}$ and the filter frequency to $5 \mathrm{kHz}$, because at this point the experimental conditions were already adapted to the experiments in the presence of ampicillin. For each membrane, a new glass chip was used.

Membranes formed with this method were found to be stable for no more than $30 \mathrm{~min}$ and the peak-to-peak noise at $+40 \mathrm{mV}$ and $1 \mathrm{kHz}$ filter frequency was 1-2 pA.

\subsubsection{Black lipid membranes technique using the BLM setup}

The BLM setup (Figure 3.12) was a self-made replica of the setup in the Meinecke lab, constructed with the help of Niels Denkert and Michael Meinecke (Meinecke group, department of Cellular Biochemistry, University Medical Center Göttingen). The measuring chamber (see Figure 3.12 and Figure 3.13) consists of two cylindrical PTFE half chambers, separated by a PTFE foil (DF100 cast film, Saint-Gobain Performance Plastics, Rochdale, Great Britain) containing a small aperture $(\sim 50 \mu \mathrm{m})$. On the other side of chamber, a glass slide $(d=21 \mathrm{~mm}$, $1 \mathrm{~mm}$ thick, Glasbearbeitung Henneberg \& Co., Martinroda, Germany) fixed by a seal ring enables light transmission. The half chambers are held together by an aluminum shell, serving as faraday cage. Each chamber has a small hole for the electrode and a larger one to add so- 
lutions. The entire measuring chamber including the aluminum shell was placed on an aluminum plate (grounding) on the electric stirrer. The headstage CV 203BU (Axon Instruments, Union City, CA, USA) was used as preamplifier, lamp and transmitted light microscope enabled visualization of the aperture in the PTFE foil. The stirrer SPIN-2 and the lamp SUN-1, both including separate controller (Warner Instruments LLC, Hamden, CT, USA), are specially isolated, so that they do not disturb the measurements. Every component is grounded and placed in a large faraday cage (aluminum grid). If asymmetric buffer conditions are desired, two syringes and tubes can be used to exchange buffer in one half chamber during experiment without rupturing membrane (perfusion unit). However, the perfusion unit was not used during this project.

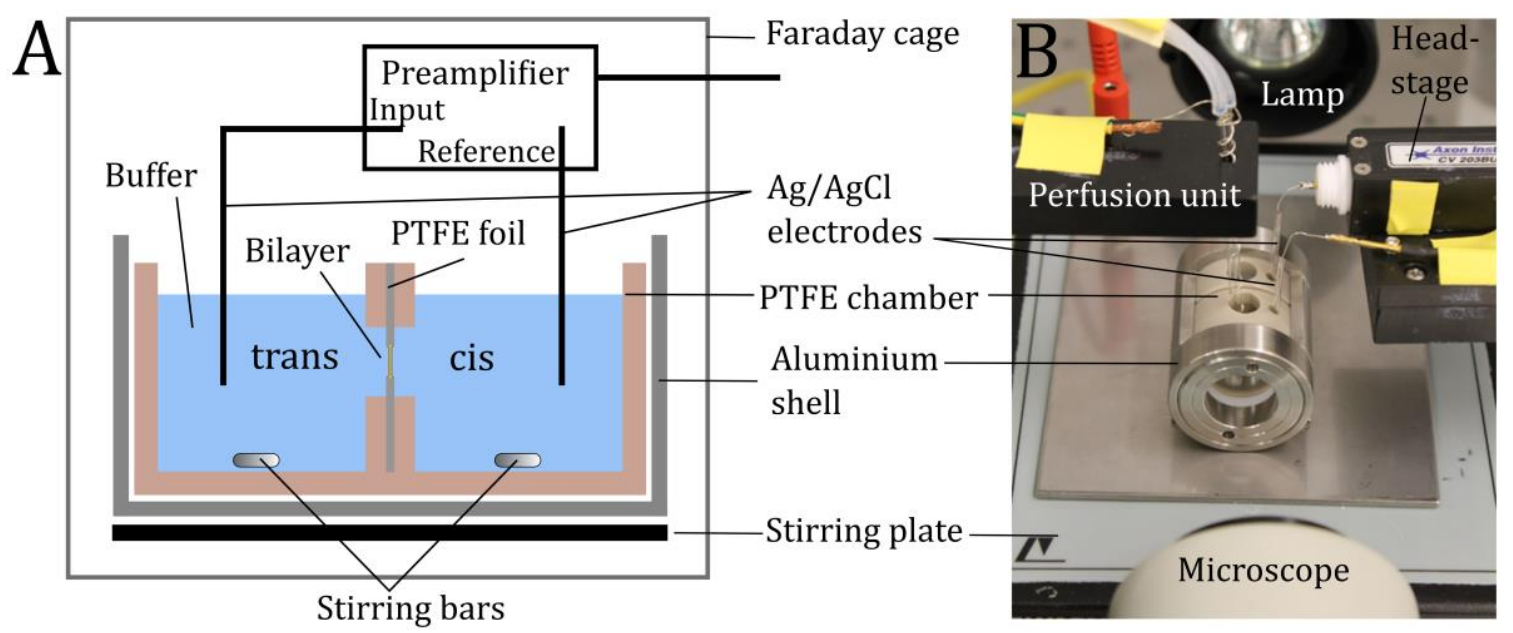

Figure 3.12. Patch-clamp experiment using the self-constructed BLM setup. Scheme (A) and image (B) of the setup. The measuring chamber consists of two PTFE half chambers, separated by a PTFE foil and surrounded by an aluminum shell. A transmitted light microscope enables the visualization of the bilayer formed over the aperture in the PTFE foil. The front half chamber is connected to the reference electrode and thus is referred to as the cis chamber.

As main amplifier the Axopatch 200B (Axon Instruments, Union City, CA, USA), including a four-pole low pass Bessel filter, and as A/D converter the Digidata 1322A (Axon Instruments, Union City, CA, USA) were used. Clampex version 10.4.0.36 from the pClamp 10.4 software package (Molecular Devices, Sunnyvale, CA, USA) was used to conduct the measurements.

\section{Realization}

The PTFE foil was prepared in advance. It was cut into $1.5 \times 1.5 \mathrm{~cm}$ pieces and punctured in the center using a needle. The resulting hole was widened using a self-made spark gap in the Meinecke lab.

Electrodes were prepared as described in Chapter 3.6.3.1. Additionally, they were surrounded by agarose to minimize noise. $2 \%$ Agarose (NEEO ultra quality; Sigma-Aldrich, 
Taufkirchen, Germany) in $2 \mathrm{M} \mathrm{KCl}$ were heated while stirring until the solution was liquid. The electrodes were fixed in small glass capillaries (micro hematocrit capillaries type B, $d_{\text {in- }}$ side $=1.1-1.2 \mathrm{~mm}$, VWR International GmbH, Darmstadt, Germany), dipped into the liquid agarose until the agarose was sucked into the capillary and immediately stored in measuring buffer to prevent the agarose from drying out.

To prepare the measuring chamber (see Figure 3.13), a glass slide was inserted on each half chamber, covered with a piece of Parafilm ${ }^{\circledR}$ slightly larger than the glass slide and fixed using a PTFE seal ring. Finally, the Parafilm ${ }^{\circledR}$ covering the glass slide was cut out, leaving the Parafilm ${ }^{\circledR}$ between the seal ring and the glass to avoid leakage. Vacuum grease was spread on the other side of both half chambers, the PTFE foil was fixed without wrinkles to one chamber (aperture centered) and the edges were covered with grease. The half chamber was inserted into the aluminum shell, followed by the second one, so that the greased ends faced each other and fixed by screwing on the lid of the aluminum shell.

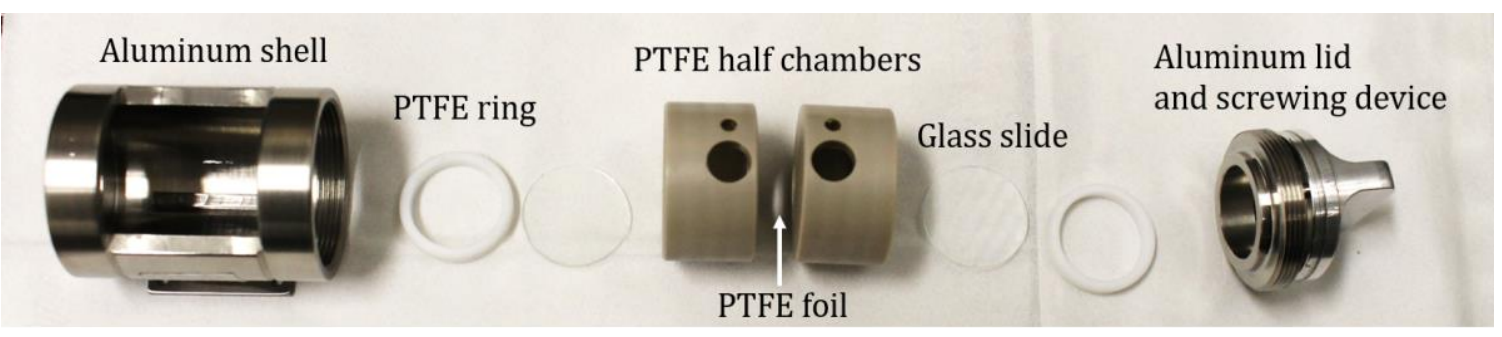

Figure 3.13. Preparation of the BLM measuring chamber. The PTFE half chambers are closed with a glass slide and a seal ring on one side and face each other on the other side, separated by a PTFE foil. The aluminum shell holds them together and serves as faraday cage.

Each half chamber was filled with $3 \mathrm{~mL}$ measuring buffer $(1 \mathrm{M} \mathrm{KCl}, 10 \mathrm{mM}$ HEPES, pH 6 or 7.5) and mounted on the electric stirrer. The electrodes were connected to the gold contacts of the headstage and immersed into the buffer. After turning on the amplifier in the whole cell mode, the overload lamp was on if a current flow through the aperture was possible. Otherwise, it was checked for air bubbles blocking the aperture (compare Figure 3.14 A and B). Looking through the microscope, lipid (DPhPC/ Chol 9:1, $60 \mathrm{mg} / \mathrm{mL}$ ) dissolved in $n$-decane was added to the aperture at the cis side of the PTFE foil (front chamber, reference electrode) using a blunt bent microliter syringe (Hamilton ${ }^{\circledR}$ TLC syringe, model 1701 RN, Sigma-Aldrich, Taufkirchen, Germany) until the aperture was completely covered (see Figure 3.14 C) and the overload lamp went off. The lipid solution was distributed on both sides of the foil by changing the buffer level using a glass pipette. After an incubation time of $30 \mathrm{~min}$, thinning of the solution by changing the buffer level led to an electrically insulating, stable bilayer (compare Figure 3.14 D and E). If the bilayer frequently ruptured, addition of more lipid solution followed by thinning was necessary. A thin bilayer is barely visible and the current signal should 
be near zero. Finally, the stability of the bilayer was checked applying voltages between +40 and $+140 \mathrm{mV}$, at which no offset of the current signal should be visible.
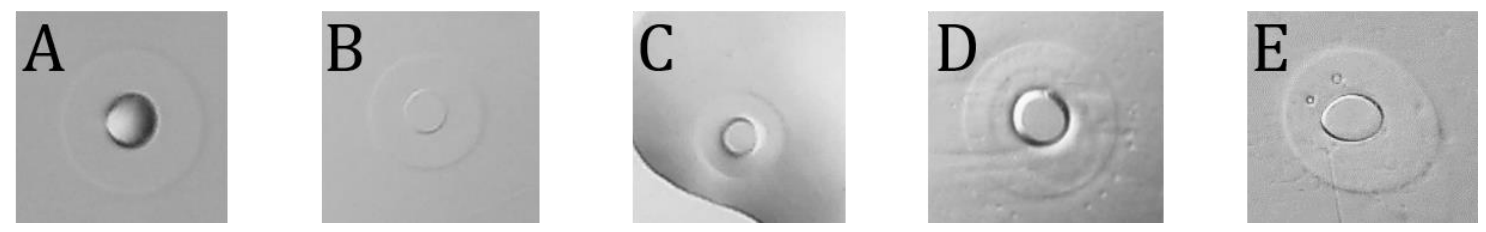

Figure 3.14 Aperture in the PTFE foil visualized using the transmitted light microscope. (A) An air bubble blocks the aperture. (B) Free aperture. (C) A drop of lipid solution covers the aperture. (D) Bilayer before and (E) after thinning.

PorB (0.02-2.2 $\mu \mathrm{M}$ in $200 \mathrm{mM} \mathrm{NaCl}, 20 \mathrm{mM}$ Tris, $0.1 \%(w / w)$ LDAO, pH 6 or 7.5) was added to the cis side using a bent, blunt Hamilton ${ }^{\circledR}$ TLC syringe followed by stirring until an insertion was visible by an abrupt increase of the current level. The stirrer was immediately turned off to avoid more insertions.

The desired volume of ampicillin (25 mM in $1 \mathrm{M} \mathrm{KCl}, 10 \mathrm{mM}$ HEPES, pH $7.5 \mathrm{or} \mathrm{pH}$ 6) was added to either both sides of the aperture, or only the trans side (back chamber, input electrode) for control using a microliter pipette (Eppendorf AG, Hamburg, Germany), followed by stirring gently.

Before starting the recordings, the faraday cage was closed gently. Current traces were recorded continuously ( $60 \mathrm{~s}$ each) at +40 to $+120 \mathrm{mV}, 50 \mathrm{kHz}$ sampling rate and $5 \mathrm{kHz}$ filter frequency at $20^{\circ} \mathrm{C}$. Membranes formed with this method were found to be stable for up to $6 \mathrm{~h}$ and the peak-to-peak noise at $+40 \mathrm{mV}$ and $5 \mathrm{kHz}$ filter frequency was about $20 \mathrm{pA}$.

\subsubsection{Black lipid membranes technique using the Orbit 16}

The Orbit 16 (Nanion Technologies, München, Germany, Figure 3.15 C) contains 16 high-resolution amplifiers eONE (elements SRL, Cesena (FC), Italy) and enables formation and measurement of 16 BLMs in parallel. A micro electrode cavity array (MECA) chip (Ionera Technologies $\mathrm{GmbH}$, Freiburg, Germany, Figure 3.15 A and B) is used as planar glass substrates. It contains 16 cavities with apertures $(d=50 \mu \mathrm{m})$ and input electrodes $(\mathrm{Ag} / \mathrm{AgCl}$ micro electrodes) connected over the contact board and gold contacts to the Orbit 16 . The cylindric PTFE chamber with seal ring holds the buffer solution in which the reference electrode is immersed. A magnetic stirrer implemented in the Orbit enables bilayer formation. The metal shell surrounding the entire Orbit 16 serves as faraday cage. The EDR software version 3.3.8 (elements SRL, Cesena (FC), Italy) was used for recordings. 


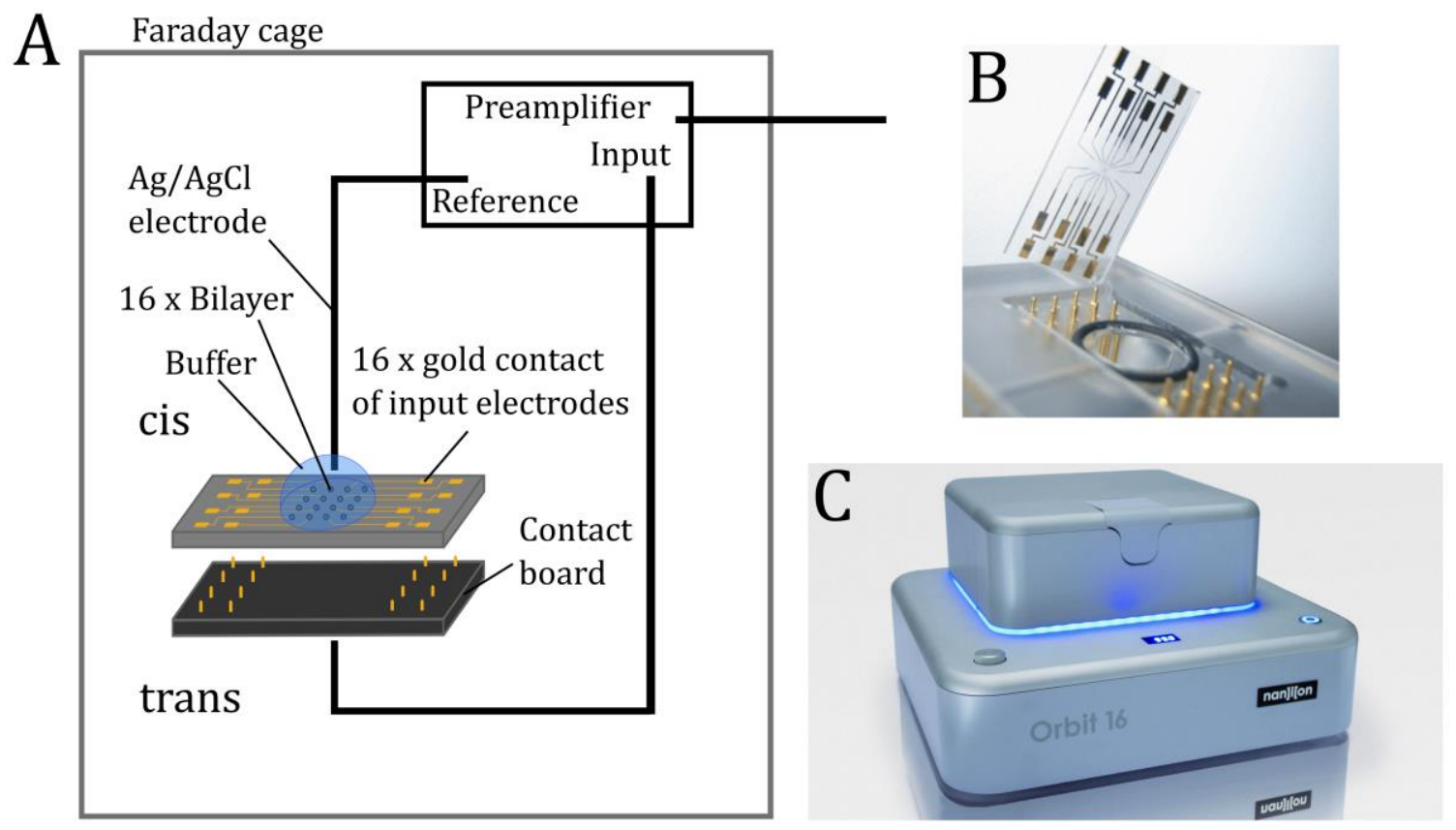

Figure 3.15. Patch-clamp experiment using the Orbit 16. Scheme of the experimental setup (A). 16 cavities with corresponding contacts for the signal electrodes enable the formation of 16 bilayers in parallel. Lipid material and protein is added to the cis side (reference electrode). Micro Electrode Cavity Array (MECA) chip used as planar glass substrate (B). Image of the Orbit 16 (C).[108]

\section{Realization}

In contrast to experiments using the Port-a-Patch and BLM setup, a renewing of the electrodes as described in Chapter 3.6.3.1 was not necessary after each measurement. They only needed to be cleaned thoroughly. The input electrodes were exchanged automatically with each measuring chip and the reference electrode could be treated as described if the signalto-noise ratio got worse.

The MECA chip was rinsed with water, ethanol and isopropanol, dried under nitrogen stream, and finally heated to $60{ }^{\circ} \mathrm{C}$ for $15 \mathrm{~min}$ using a heating plate. Contact board and MECA chip including PTFE chamber, seal ring and stirring bar were inserted into the Orbit 16 . After addition of $150 \mu \mathrm{L}$ measuring buffer $(1 \mathrm{M} \mathrm{KCl}, 10 \mathrm{mM}$ HEPES, pH 7.5), a triangular voltage profile was applied to check if the apertures of each cavity were free. Due to the overload clipping of the signal, free apertures show a rectangular current response. In case of some blocked apertures, the MECA chip was either cleaned again or exchanged.

Subsequently, 0.4-0.8 $\mu$ lipid solution (DPhPC, $1 \mathrm{mg} / \mathrm{mL}$ in $n$-decane) were added to the chip near the surface and distributed over all apertures using the magnetic stirrer. This way, the bilayers were formed (painting). The current signal now did not show an overload anymore. If bilayers or multilayers were formed was distinguished determining the capacitance: 7-10 $\mathrm{pF}$ were found for a bilayer, 0-3 $\mathrm{pF}$ for a multilayer or clogged aperture. Further indication 
was obtained by destroying the membranes using a voltage pulse, as only bilayers were destroyed. Signals of clogged apertures were turned off using the software. After repainting the bilayers and checking their stability by applying voltages between +40 and $+140 \mathrm{mV}$, the recordings were started.

Volumes of $0.1 \mu \mathrm{L}$ PorB solution $(0.2 \mu \mathrm{M}$ in $200 \mathrm{mM} \mathrm{NaCl}, 20 \mathrm{mM}$ Tris, $0.1 \%(w / w)$ LDAO, pH 7.5) were added stepwise until insertions were visible in several apertures. Application of a voltage pulse of $+60 \mathrm{mV}$ was often used to improve the insertion efficiency.

Data was recorded continuously in one file at voltages of +40 to $+100 \mathrm{mV}$, a sampling rate of $10 \mathrm{kHz}$ and a filter frequency of $5 \mathrm{kHz}$ at $25^{\circ} \mathrm{C}$. The peak-to-peak noise at $+40 \mathrm{mV}$ and $5 \mathrm{kHz}$ filter frequency was determined as $0.02 \mathrm{pA}$.

\subsubsection{Data analysis}

\subsubsection{Single channel measurements without antibiotic}

Channel gating is characterized by abrupt, step-like increases and decreases in the current amplitude (Figure 3.16). This can happen between the closed and one open state or between two open states. The current amplitude between the states can be divided by the applied voltage to obtain the conductance of the channel. The time the channel spends in the open state is the dwell time.
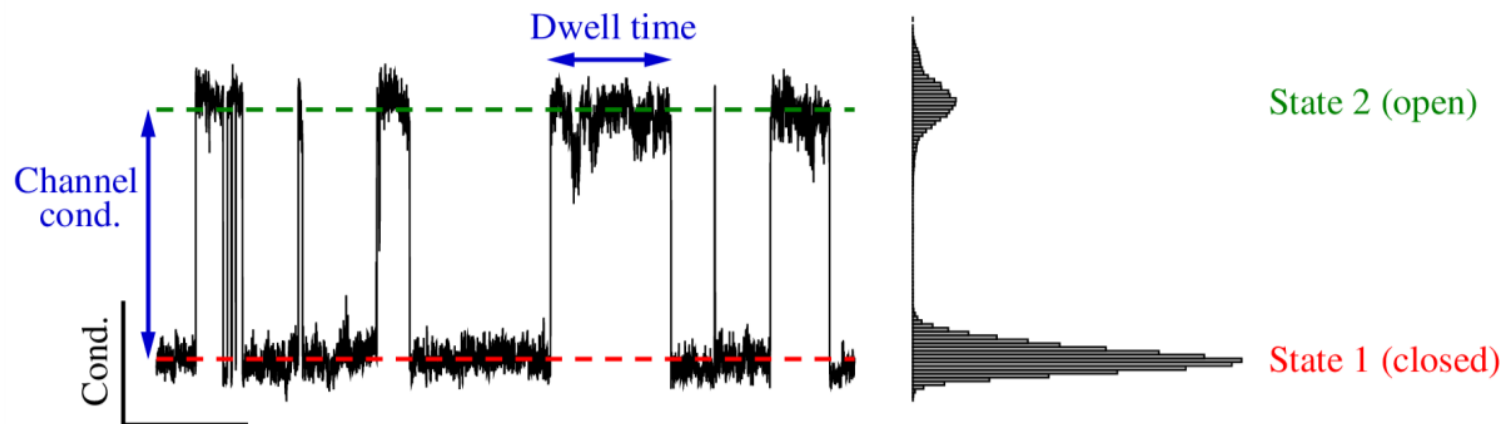

Time

Figure 3.16. Analysis of a conductivity trace showing channel gating. The two states marked in red (closed) and green (open) are visible in the trace and the corresponding point amplitude histogram. From the traces, the channel conductance (cond.) and its dwell time are accessible.

Such current traces can be analyzed in different ways. Conductivity event histograms yield the values of the main conductance states and point amplitude histograms can be used to determine the probabilities of the different states. The latter give information about the conductance states as well. 


\section{Conductivity event histograms}

The Software Clampfit version 10.4.0.36 from the pClamp 10.4 software package (Molecular Devices, Sunnyvale, CA, USA) and the mode single channel search were used to obtain the data needed for the conductivity event histograms. First, the desired range of each current traces was chosen and the different conductivity levels were determined. The software detects jumps in current signal between these levels and the user decides whether to take the event or not. As this analysis by hand is very time consuming, one can let the software take all detected events without verification by the user. This method is faster, but less accurate, because many artefacts or membrane fluctuations are taken as real gating events. In this work, solely the analysis by hand was performed.

To avoid doubling of the events, only steps to an upper level were included in the further evaluation. Using either MATLAB R2014a (The MathWorks, Inc., Natick, MA, USA) or Origin Pro 8.5G (OriginLab Corporation, Northampton, MA, USA), the conductance values were plotted as an event histogram and fitted using Gaussian distributions (Equation 3.10).

$$
\mathrm{f}\left(G_{\mathrm{i}}\right)=\frac{A}{\sigma_{\mathrm{G}_{\mathrm{i}}} \sqrt{2 \pi}} \cdot \exp \left(-\frac{\left(G_{i}-G\right)^{2}}{2 \cdot \sigma_{G}^{2}}\right)
$$
$G_{\mathrm{i}}$ : conductivities
A: area below the curve
$G$ : estimated value of the conductivity
$\sigma:$ standard deviation

The number of bins was chosen according to Freedman and Diaconis (Equation 3.11).[109]

$$
\delta=\frac{2 \cdot \operatorname{IQR}(\mathrm{x})}{n^{1 / 3}}
$$

$\delta$ : interval width

IQR: interquartile width

$n$ : number of events

\section{Point amplitude histograms}

To obtain the point amplitude histograms, the current traces were analyzed using a MATLAB script written by Jeremias Sibold (Steinem group, Institute of organic and biomolecular chemistry, University of Göttingen). The script Gaussfit2.m (needs the script abfload.m, https://de.mathworks.com/matlabcentral/fileexchange/6190-abfload) transfers the current values into conductivities and plots all data points as point amplitude histogram. The user is asked for a minimal and maximal conductivity value to determine the range for further evaluation and the number of maxima that should be fitted. Subsequently, the histogram is fitted using multiple Gaussian distributions (see Equation 3.10) and the user has to decide if the fit is suitable. If not, there are two possibilities: run the evaluation again with an adjusted 
analysis range, or determine start values of amplitude, center and full width at half maximum for each maximum to improve the fit. If the fit is reasonable, the script provides the centers of the maxima, which can be used to normalize the value of the closed channel to zero. Finally, the areas of the maxima are calculated. They correspond to the probability of the different states (open, closed...).

The script GaussfitN.m can be used in the same way to analyze the normalized values and export the data needed to plot the graphs with a different program, e.g., GLE. The scripts can be found in the appendix (Chapter 7.7.1).

When comparing the results, it must be taken into account that the values strongly depend on the chosen section of the conductivity trace. Since in solvent-free membranes the porins show only short periods of gating between long periods spent in the closed state, the open probability is influenced by the length of the section taken before and after the gating-period. In order to enable the best comparability, the traces were cut directly before the first and after the last event. As a result, the determined open probabilities are higher than the average of the whole conductivity trace. Nevertheless, this approach is necessary for the calculation of open probabilities, because it cannot be distinguished between a channel inserted in the membrane but closed for a long time and no channel inserted at all. As a consequence, the analyzed traces recorded on solvent-free membranes consist of varying numbers of data points. Determining the open probability of each trace and averaging would not consider this, which is why all traces were combined and evaluated as one data set. Therefore, no error can be indicated for the open probabilities in solvent-free membranes. The traces recorded on BLMs, however, showed continuous gating, so that all analyzed traces consisted of the same amount of data points. In this case, each trace was analyzed separately and an average value was calculated. The indicated errors are the standard deviation. The same approach was applied for the determination of the voltage dependent conductivities, which were as well determined from the point amplitude histograms.

\section{Noise analysis}

The noise analysis was performed using the MATLAB script PorB.m (appendix, Chapter 7.7.2) written by Ingo Mey (Steinem group, Institute of organic and biomolecular chemistry, University of Göttingen). It needs the scripts abfload.m, dtr.m (appendix, Chapter 7.7.2) and peakfinder.m (https://de.mathworks.com/matlabcentral/fileexchange/25500-peakfinder-x0-sel--thresh--extrema--includeendpoints--interpolate-)). The user selects the abf-file to be an- 
alyzed, determines a minimal and maximal threshold (percentage deviation from the baseline) and the number of steps in which the threshold range should be analyzed. Finally, the selectivity (sel) of the peakfinder has to be chosen, larger values meaning higher selectivity. First, the script determines the baseline (median) and then applies systematically increasing thresholds. All data points lying beyond the threshold below and above the baseline are chosen. The peak maxima within these data points are detected and a peak frequency is calculated (see Figure 3.17). Finally, this frequency below (down) and above (up) the baseline is plotted against the applied threshold. The higher this frequency, the higher the noise of the current trace. Of note, the script can only be applied to traces without channel gating, as the latter would be defined as points deviating from the baseline and many peaks would be detected, falsifying the result.

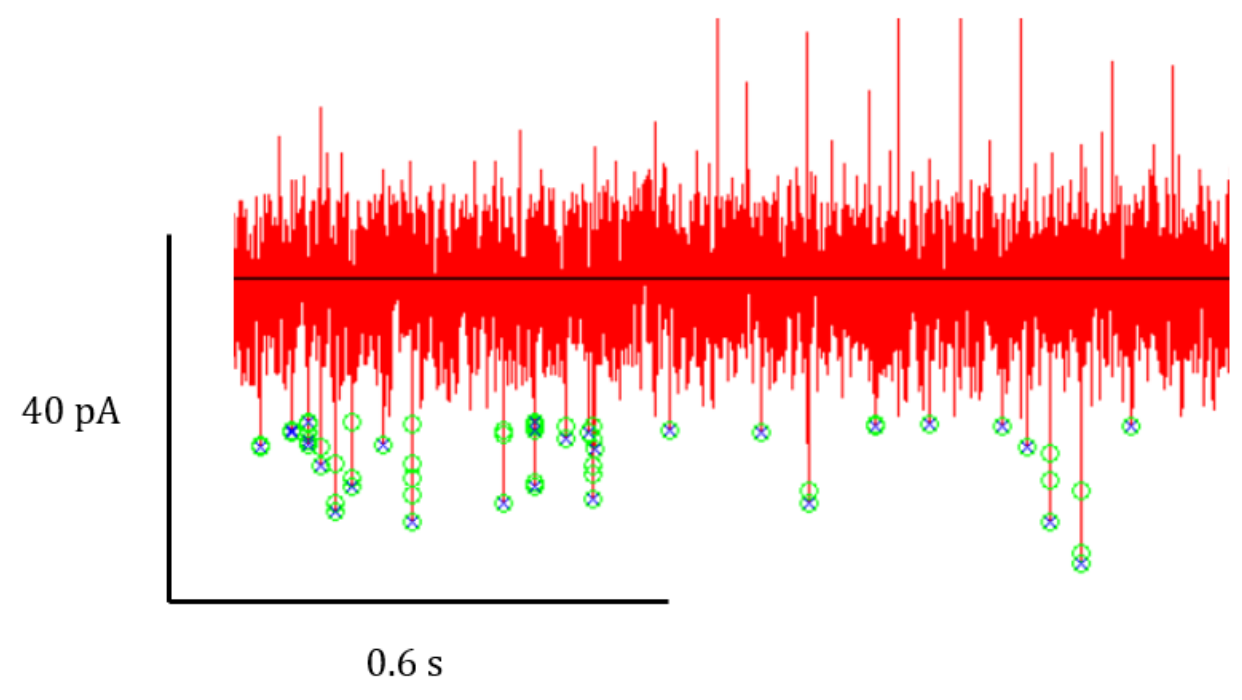

Figure 3.17. Noise analysis using the MATLAB script PorB.m. The data points are plotted in red and the calculated baseline is depicted in black. Data points found beyond the chosen threshold (below the baseline in this example) are marked with green circles and the peaks detected among them are tagged with a blue " $\mathrm{x}$ ".

\subsubsection{The analysis routine JULES}

An antibiotic molecule bound to a porin channel can block the pore and reduce the ion flux. In electrophysiological measurements, these blockages are visible as short interruptions of the open channel conductance. The blockage events of PorB detected in the presence of ampicillin (see Figure 3.18 A and B) occurred on an extremely short timescale compared to antibiotic blockages described in literature.[52,55] Therefore, the common analysis method of fitting a Lorentzian distribution to a power spectrum could not be applied to the obtained data (see Chapter 4.3.1), because too few data points exist at the blocked level to detect a difference between traces with and without ampicillin (see Figure 4.31). This is further compli- 
cated by the smoothing effect of the four-pole low pass filter applied during the measurements. At an applied cutoff frequency of $f_{\mathrm{c}}=5 \mathrm{kHz}$, the filter rise time is $t_{\mathrm{R}}=70 \mu \mathrm{s}$. Signals of durations below this value are smoothened so strongly by the filter that the values determined during analysis appear lower than the original signal without filter would be (Figure $3.18 \mathrm{~B}$ ).

Instead, a new analysis routine called JULES (IUmp Local dEcconvolution Segmentation filter) developed and applied by Florian Pein (Munk group, Institute for Mathematical Stochastics, University of Göttingen) was used.[110] This routine enables model-free idealizations of events below the filter length by combining multiresolution techniques and local deconvolution.

The $\mathrm{R}$ function jules from the package clampSeg (https://CRAN.Rproject.org/package=clampSeg, version 1.0-1) is applied with default parameters. The detection limit of JULES was determined via simulations. At all signal-to-noise ratios, events with residence times of $80 \mu$ s and longer are detected reliably. As the signal-to-noise ratio varies with the ampicillin concentration and the applied voltage, shorter events might be missed in some measurements, but detected in others, which would manipulate the results. Further, events occurring on a longer time scale cannot be distinguished from channel gating events. Thus, the analysis is restricted to events between 80 and $200 \mu$ s.

The idealized blockage amplitudes, i.e., the conductivities blocked by ampicillin, are plotted in an event histogram together with a kernel density estimation using a Gaussian kernel (bandwidth 0.05, $\mathrm{R}$ function bkde of the KernSmooth package, version 2.23-15, https://CRAN.R-project.org/package=KernSmooth) and show one isolated maximum (Figure $3.18 \mathrm{C}$ ). The mean blockage amplitude is determined using a Gauss fit and all events not belonging to this maximum are neglected during further analysis, as they cannot be related to the blockage by ampicillin.

The events between 80 and $200 \mu$ s within the maximum of the blockage amplitudes are then used to determine the times between two events and from these the frequency of blockage, as well as the residence times of ampicillin, i.e., the duration of the events. Figure $3.18 \mathrm{D}$ shows the event histogram of the residence times and their exponential decrease. For the calculation of the blockage frequencies, the mean time between two blockage events is multiplied with the number of open channels - determined by dividing the conductance of the non-blocked state by the blockage amplitude - and the probability that an event is not missed. The inverse of this rescaled time is the blockage frequency. For the residence times, 95\%confidence intervals are calculated by a normal approximation, while for the frequencies an exact confidence interval is determined. 
A

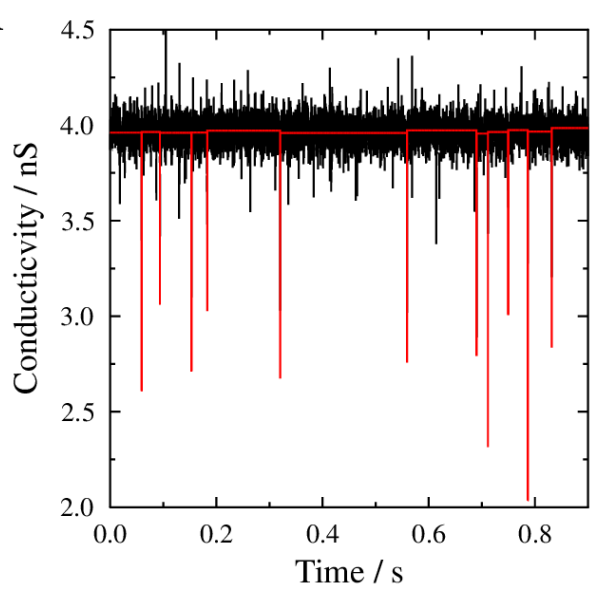

$\mathrm{C}$

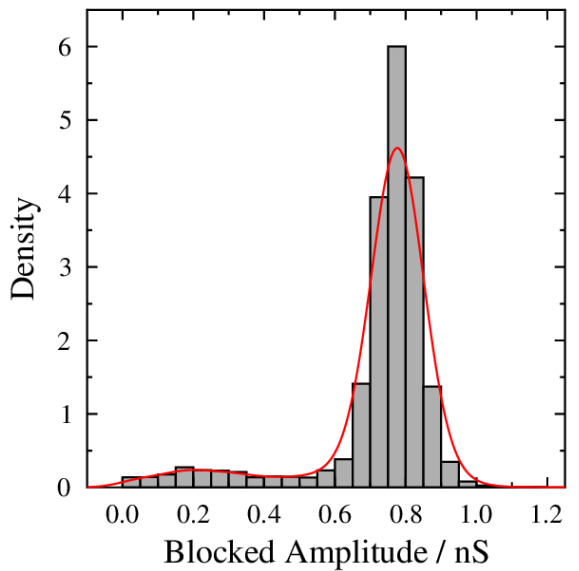

$\mathrm{B}$

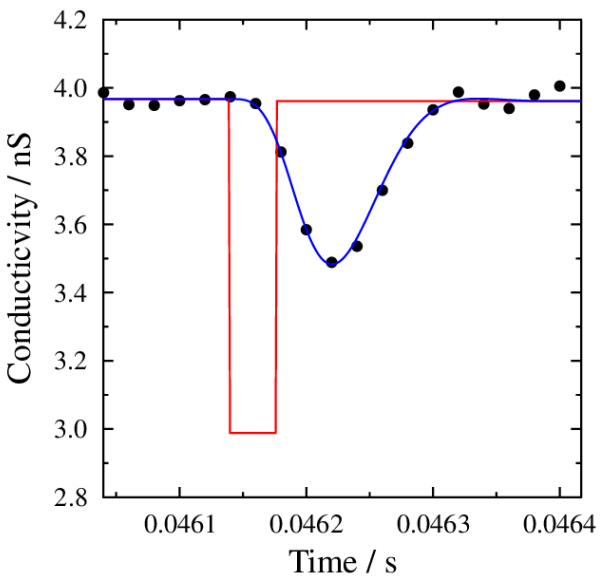

$\mathrm{D}$

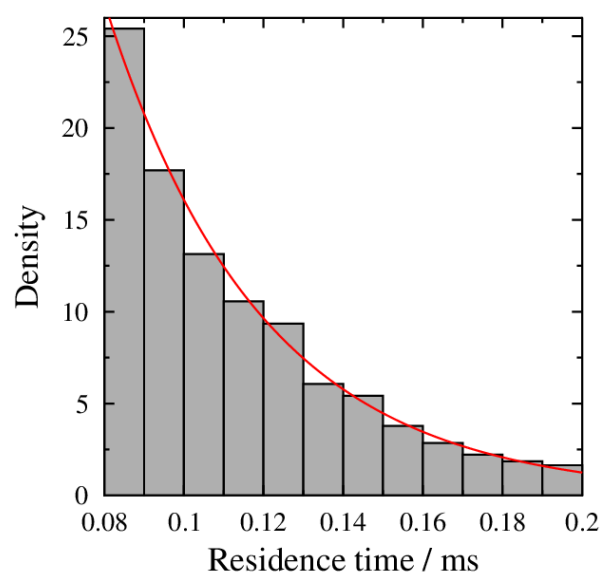

Figure 3.18. The analysis routine JULES. Original conductivity trace (black) and signal reconstruction (red)(A). Zoom-in of one blockage event (B). The recorded data points are shown in black and the reconstructed signal in red. After filtering (blue line) the reconstructed signal, it fits well to the originally recorded data, confirming the success of the idealization. Event histogram and kernel density estimation using a Gaussian kernel of the determined blockage amplitudes showing one isolated maximum (C). Event histogram of the ampicillin residence times and exponential fit (red line) considering missed events (D).

\subsection{Molecular dynamics simulations}

Molecular dynamic (MD) simulations were introduced in the $1950 \mathrm{~s}^{[112,113]}$ and the first simulation of a protein was reported in 1977.[114] Since then, MD simulations have become a pivotal tool to comprehend the structure and function of biological macromolecules on their physical basis.[115]

MD simulations calculate the physical movements of every atom of a system revealing the dynamics of the system from which time averaged properties can be obtained.[116] Atoms are treated as charged spheres connected by springs whose behavior is defined by the potential 
energy function of the system also known as molecular force field.[117] The standard for simulations of biomolecular systems are more than 100000 atoms and a time scale of nanoseconds to microseconds.

In the last two decades, the permeation of antibiotics through porins has been intensively studied using MD simulations. ${ }^{[52,53,56,60,63,118,119]}$ An antibiotic molecule entering a pore is a rare event compared to the time scale of MD simulations, ${ }^{[28]}$ therefore some specialized methodologies (enhancing sampling techniques) have been used. The most common enhanced sampling methods used for this purpose are steered molecular dynamics and metadynamics.[118,120] In Steered molecular dynamics simulations, the passage of the molecule through the channel is forced by attaching a probe that steers the molecule and thus accelerating the translocation process. Metadynamics simulations bias the system to sample all the possible configurations of the process of interest leading to an accurate free energy description of the process.

The MD simulations on PorB and ampicillin were performed by Salomé Llabrés and Ulrich Zachariae (Computational Biology, School of Life Sciences, University of Dundee, UK). Docking calculations were used to study the binding mode of ampicillin and the permeation of ampicillin through the eyelet of PorB was explored via steered molecular dynamics simulations. Finally, computational electrophysiology simulations were performed to investigate the influence of an applied voltage on the system.

Set up of the PorB system

PorB wt (PDB-ID: 3VY8) was modelled using the X-ray structure obtained by Kattner et al.[62] and the structure of the G103K mutant was obtained from Christof Kattner (ZIK HALOmem, Membrane Protein Biochemistry, Martin-Luther-University Halle-Wittenberg) and Mikio Tanabe (Institute of Materials Structure Science, Structural Biology Research Center, Ibaraki, Japan, unpublished results). PorB trimers were embedded into a preequilibrated $160 \times 160 \AA^{2}$ 1-palmitoyl-2-oleoyl-sn-glycero-3-phosphocholine (POPC) bilayer composed of 942 molecules using the GROMACS utility membed. ${ }^{[121,122]}$ At both sides of the bilayer, a $25 \AA$ A thick layer of SPC/E (extended simple point charge) water molecules was generated, including $\mathrm{Na}^{+}$and $\mathrm{Cl}^{-}$ions to obtain an ionic strength of $1 \mathrm{M}$. 57000 water, $1866 \mathrm{Na}^{+}$and $1896 \mathrm{Cl}$ - ions were present in the aqueous solution. For PorB, virtual sites for hydrogen atoms ${ }^{[123]}$ and the Parm99SB force field $[124,125]$ were applied. The parameters of the POPC molecules were set using the lipid parameters according to Berger et al.,[125,126] water molecules were modelled 
applying the SPC/E water model and for the counter ions, Joung and Cheatham parameters ${ }^{[127]}$ were utilized. The parametrization of the zwitterionic ampicillin molecule was achieved applying the gaff force field ${ }^{[128]}$ together with restrained electrostatic potential (RESP) (HF/6-31G(d)) charges ${ }^{[129]}$ as realized in the Antechamber module of the AMBER12 software package.[130]

\section{Docking calculations}

Docking calculations were performed using GOLD ${ }^{[131]}$ and $\operatorname{rDock}^{[132,133]}$ software packages. The structural models of PorB wt and the mutant were the same as described above. 100 docking runs were carried out with the zwitterionic and the anionic form of ampicillin, respectively. While the conformational flexibility of ampicillin around rotatable bonds is provided by GOLD and rDock, PorB was kept rigid. The output docking modes were analyzed by visual inspection and considering the docking scores.

\section{Molecular dynamics simulations}

The GROMACS molecular dynamics package version $5.1 .5^{[134]}$ was applied for the molecular simulations. The geometry was minimized in four cycles consisting of 3500 steps of steepest descent algorithm followed by 4500 steps of conjugate gradient. Thermalization of the system was carried out in 6 steps of $5 \mathrm{~ns}$, gradually increasing the temperature from $50 \mathrm{~K}$ to 320 $\mathrm{K}$, restraining the protein with a force constant of $10 \mathrm{~kJ} / \mathrm{mol} \AA^{2}$. Equilibration of the system was performed for 100 ns and production runs consisted of 200 ns long trajectories. A constant temperature was ensured by separately subjecting solvent, protein, and membrane to a weak coupling ( $\mathrm{t}=0.1 \mathrm{ps}$ ) to a temperature bath of $320 \mathrm{~K}$ using the velocity-rescale thermostat of Bussi et al. ${ }^{[135]}$ Semi-isotropic Berendsen coupling[136] was applied to keep the pressure constant at 1 bar. The smooth particle mesh Ewald method[137] was used to calculate long-range electrostatic interactions beyond a short-range Coulomb cut-off of 10 Å. LennardJones interactions were also cut-off at $10 \AA$ A. The system was constrained applying the LINCS algorithm ${ }^{[138]}$ and angles and bond lengths of water molecules were restrained by the SETTLE algorithm. ${ }^{[139]}$ Periodic boundary conditions were used and the integration time-step was set to 4 fs applying the Berger lipid model and using the virtual sites.

\section{Computational electrophysiology simulations}

A double bilayer system was set by duplicating each system along the $z$-axis. The transmembrane potential range from $\pm 130, \pm 350$ and $\pm 500 \mathrm{mV}$ was produced using ionic imbalances 
from 4, 8 and $12 \mathrm{Na}^{+}$between the aqueous compartments, as already performed by Kutzner et al. ${ }^{[140]}$ Production runs consisted of 200 ns-long trajectories for the systems without ampicillin and of 400 ns-long trajectories for the ampicillin-bound systems. The GROMACS utility gmx potential was used to calculate the applied membrane potential, overlapping 20-ns time frames.

Analysis of the molecular dynamics trajectories

Distances, root mean square deviations (RMSD), flux of water molecules and computational conductance values were analyzed applying MDAnalysisi141] and MDtraj.[142]

Steered molecular dynamics simulations

The ampicillin centre-of-mass was pulled across the constriction regions along the $z$-axis of PorB by a moving harmonic potential with a force constant of $5 \mathrm{kcal} / \mathrm{mol} \AA^{2}$ and at a constant velocity of $5 \AA /$ ns. Two sets of 30 replicates were carried out in each direction starting from the ampicillin bound state. 


\section{RESUlTS AND DisCUSSION}

In this project, the bacterial porin PorB from Neisseria meningitidis was electrophysiologically investigated in absence and presence of the $\beta$-lactam antibiotic ampicillin. The wild type porin as well as the two mutants G103K and G103D were characterized to investigate the influence of the point mutations on the pore properties and the interaction between porin and antibiotic. Porins are the main entrance pathway for antibiotics to bacterial cells. As the mutations of PorB are situated in the key region for antibiotic binding and permeation, they might provide further insights on mechanisms leading to an increased resistance of Neisseria to antibiotics. To understand the electrophysiological results on a molecular level, molecular dynamics simulations were performed by Salomé Llabrés and Ulrich Zachariae (Computational Biology, School of Life Sciences, University of Dundee, UK).

\subsection{Characterization of the protein stock solutions using a no-SDS PAGE}

In order to ensure that PorB remains in its trimeric structure after dilution, the stock solutions were examined using a polyacrylamide gel electrophoresis (PAGE). As PorB trimers are known to dissociate into monomers in the presence of high concentrations of sodium dodecyl sulfate (SDS),[81] the detergent was only added to the running buffer, not to the gel itself or the sample loading buffer (no-SDS PAGE, Chapter 3.4). An exemplary gel is shown in Figure 4.1. PorB wt, G103K and G103D stock solutions contained $10 \mu \mathrm{M}$ protein and were denaturated at $45^{\circ} \mathrm{C}$ and $95^{\circ} \mathrm{C}$. The molecular weight of a PorB monomer is $36.7 \mathrm{kDa}{ }^{[76]}$ and the weight of a trimer, accordingly, $110.1 \mathrm{kDa}$. In the samples denaturated at $95^{\circ} \mathrm{C}$, the monomer band above $35 \mathrm{kDa}$ (orange box) is either dominant (PorB wt) or the only one visible (mutants). After heat treatment at $45^{\circ} \mathrm{C}$, however, the samples of all three proteins show besides a weak monomer and dimer band (between 55 and $70 \mathrm{kDa}$ ) a dominant trimer band between 70 and $100 \mathrm{kDa}$ (green box) 


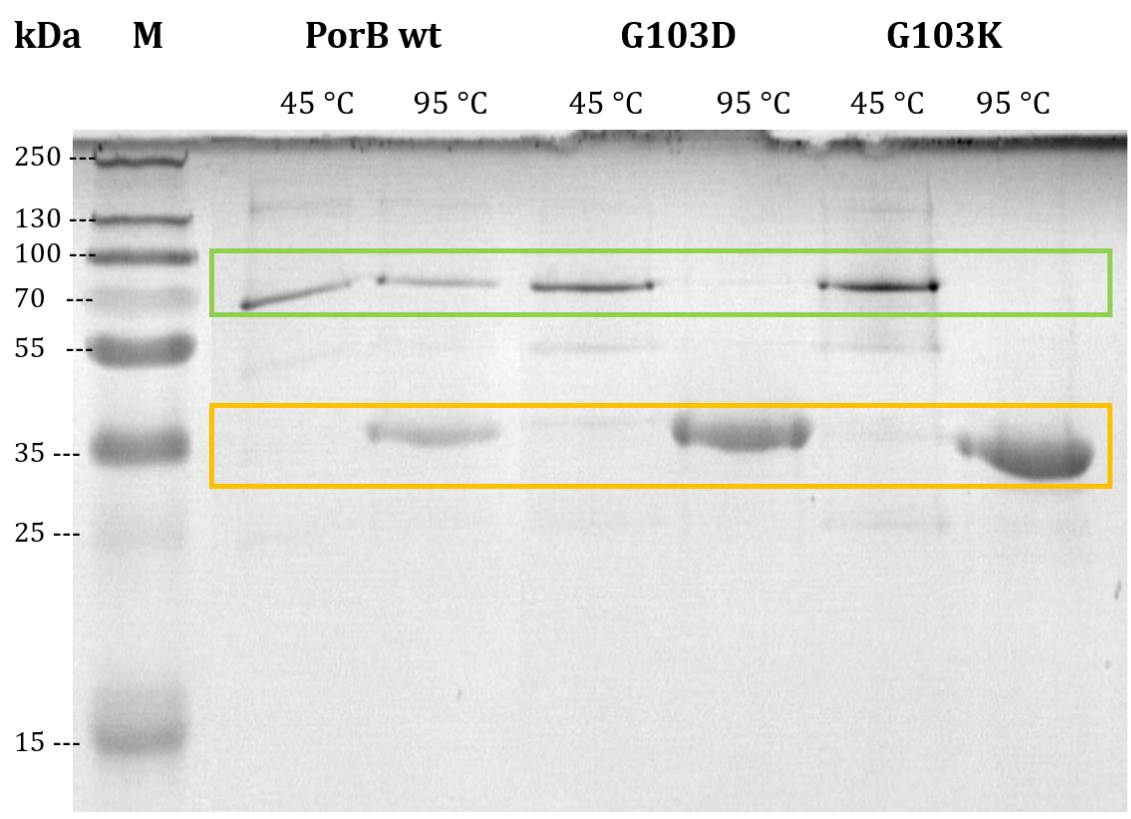

Figure 4.1. No-SDS gel of PorB wt, PorB G103D and PorB G103K. The samples were denaturated at $45^{\circ} \mathrm{C}$ and $95^{\circ} \mathrm{C}$, respectively. $\mathrm{M}$ is the molecular marker. The green box marks the trimer band and the orange box the monomer band.

Consequently, the hydrophilic interaction and the stabilizing effect of loop2 maintaining the trimeric structure (see Chapter 1.2.1) are not strong enough to withstand high temperatures, but are still intact at physiological temperatures and the temperatures present during the electrophysiological experiments $\left(\sim 20^{\circ} \mathrm{C}\right)$. Thus, it can be assumed that PorB trimers are present in the stock solutions of all three proteins and therewith as well in the experiments. Further, the mutations seem to change the interactions between the monomers somehow, as the wild type trimers are more stable at higher temperatures than the mutant trimers.

\subsection{Electrophysiological characterization in absence of ampicillin}

Prior to analyzing the interaction between ampicillin and the different porins, PorB wild type (wt), as well as the mutants G103K and G103D, were investigated in absence of the antibiotic. In order to determine the influence of the mutations on the ion conductivity and the open probability of the pore, planar patch-clamp measurements were performed in two different artificial membrane systems. Solvent-free bilayers are close to the biological membrane, but bear the disadvantage of low stability against detergents often necessary to maintain protein structure.[100] Black lipid membranes (BLMs) contain residual organic solvent which is the reason for their high stability probably caused by a lowered membrane tension.[66] On the 
other hand, protein properties and function are known to vary with the surrounding solvent.[106] Thus, the organic solvent could influence the properties of the proteins inserted into BLMs as well. Due to their different advantages, both systems were used in comparison. All experiments were performed at $20^{\circ} \mathrm{C}$ in a measuring buffer containing $1 \mathrm{M} \mathrm{KCl}$, 10 mM HEPES, pH 7.5 on bilayers composed of DPhPC/Chol 9:1. The porins were added dissolved in PorB buffer (200 mM NaCl, $20 \mathrm{mM}$ Tris, $0.1 \%(w / w)$ LDAO).

The open probability of the pores was analyzed using a MATLAB script (see Chapter 3.6.4.1) written by Jeremias Sibold (Steinem group, Institute of organic and biomolecular chemistry, University of Göttingen). In this analysis, all recorded data points (absolute counts) are plotted as a point amplitude histogram vs. the conductivity and multiple Gaussian distributions were fitted to the data. The ratio between the areas of the maxima provides the open probability of the different conductance states the porin can occupy, i.e., monomeric, dimeric or trimeric state. In addition, this evaluation yields information about the conductivity of these states. The histograms and open probabilities presented in this section result from several conductivity traces summarized in one data set.

For the conductivity event histograms the gating (i.e., transition between closed and open channel) of the pores was analyzed using the software Clampfit 10.4.0.36 (Molecular Devices, L.L.C., Sunnyvale, CA, USA) with the method single channel search (see Chapter 3.6.4.1). The transition between conductance states is detected and the conductivity difference between the states is determined and plotted as a histogram (normalized to the number of events, relative frequency). Mean conductivities are obtained by fitting multiple Gaussian distributions to the data.

The open probability shows how often the channel is in which state and which state is preferred, whereas the conductivity event histogram describes which transitions are predominant, i.e., if the monomer, dimer or trimer is mainly active. Furthermore, the point amplitude histograms generated for the analysis of the open probabilities only represent a selection of the conductivity traces while the event histograms summarize all measurements. Thus, mean conductivities obtained from the latter are more accurate.

\subsubsection{Results of solvent-free planar patch-clamp measurements}

Solvent-free membranes were formed by spreading of giant unilamellar vesicles (GUVs, see Chapter 3.5) on a planar borosilicate chip with a pore diameter of about $1 \mu \mathrm{m}$. Protein was added at an applied voltage of $+40 \mathrm{mV}$ to a stable bilayer on the cis side (side of the reference electrode). The current traces were recorded at $+40 \mathrm{mV}$ with a sampling rate of $10 \mathrm{kHz}$ (PorB 
wt, PorB G103K) or $50 \mathrm{kHz}$ (PorB G103D) and a low pass filter frequency of $1 \mathrm{kHz}$ (PorB wt, PorB G103K) or $5 \mathrm{kHz}$ (PorB G103D).

Errors of the conductivity values correspond in both analysis methods (conductivity event and point amplitude histograms) to the full width at half maximum (FWHM) of the Gaussian fit. For the open probabilities, no meaningful error can be determined. As the analyzed conductivity traces contain varying amounts of data points, a standard deviation of the values of the different traces is not appropriate (see Chapter 3.6.4.1), nor is any calculation from the error of the FWHM and the amplitude, because they depend on each other. Due to the large variation of the results of the different measurements using solvent-free membranes, the comparison of absolute open probability values is not reliable and only general tendencies are discussed.

\subsubsection{Blank measurements}

The detergent LDAO (N,N-Dimethyldodecylamine N-oxide) present in the PorB buffer can have a destabilizing effect on lipid membranes, ranging from subtle alterations in the permeability to membrane lysis, dependent on the concentration. ${ }^{[100]}$ To examine its influence on the measurements, blank samples with pure PorB buffer without protein were carried out under the same conditions as the experiments with PorB. Several minutes after the addition of the buffer, steps in the conductivity trace were observed (Figure 4.2), as would also be expected upon channel formation. However, they have only a very low conductivity, for example $0.03 \mathrm{nS}$ (Figure 4.2). The point amplitude histograms of the different blank measurements show varying distributions between the lower and upper level.
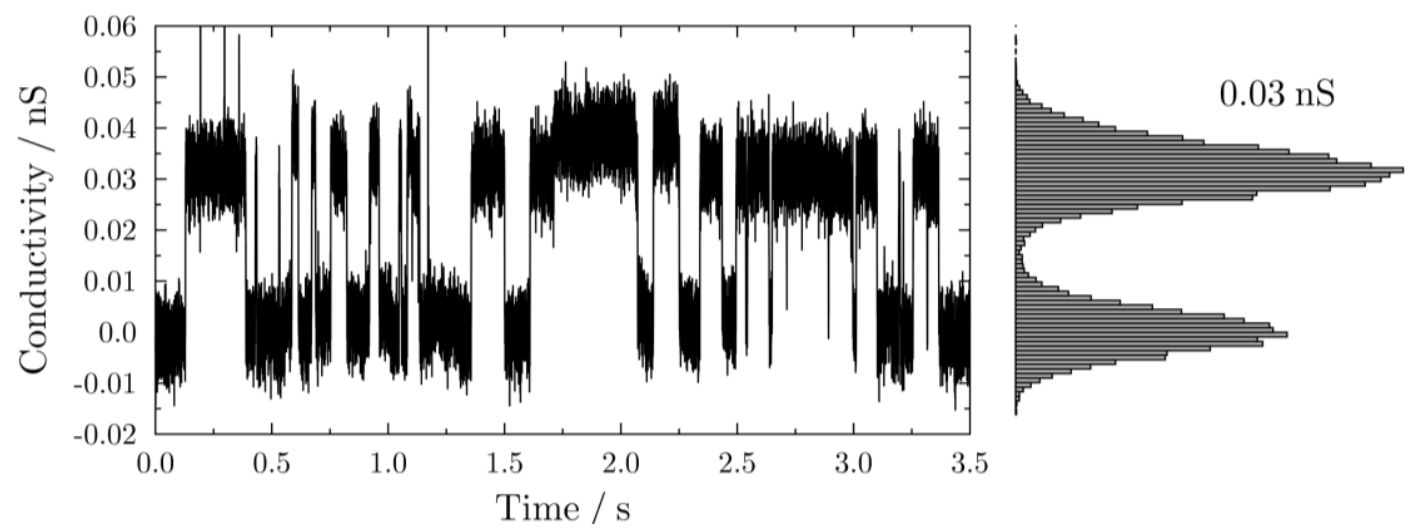

Figure 4.2. Conductivity trace of a blank sample recorded at $+40 \mathrm{mV}$ and corresponding point amplitude histogram. The conductivity of the steps is about $0.03 \mathrm{nS}$ and the histogram shows an almost even distribution between the $0 \mathrm{nS}$ and $0.03 \mathrm{nS}$ level. The measuring buffer contained $1 \mathrm{M} \mathrm{KCl}, 10 \mathrm{mM} \mathrm{HEPES}, \mathrm{pH} 7.5$ and the bilayer was composed of DPhPC/Chol 9:1. 
Blank measurements were performed distributed over all experiment days and the detected current steps were analyzed in a conductivity event histogram (Figure 4.3). Out of 2577 events, two main conductance maxima are found at $G_{1}=(0.03 \pm 0.02) \mathrm{nS}$ and $G_{2}=(0.26 \pm 0.05) \mathrm{nS}$. The value at $G_{3}=(0.54 \pm 0.17) \mathrm{nS}$ has only a very low frequency and can be neglected.

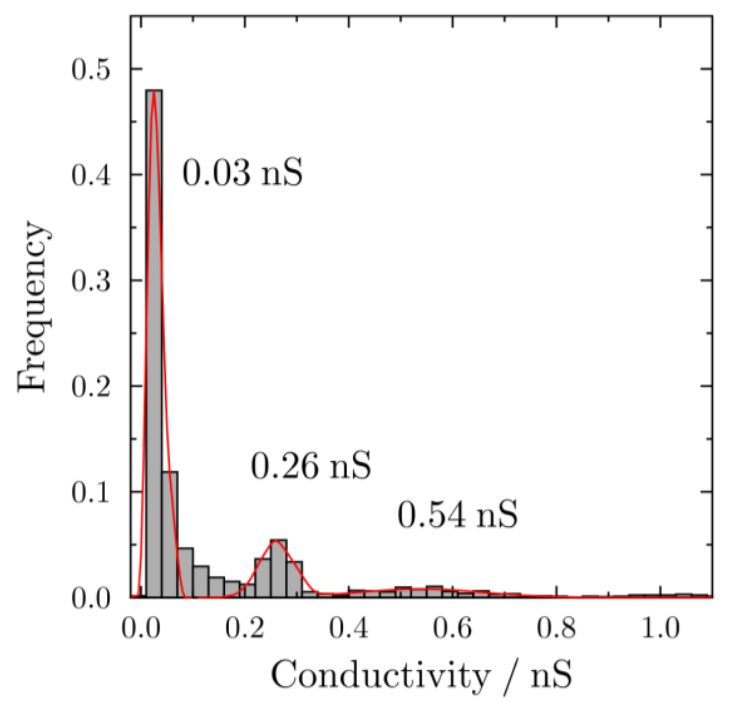

Figure 4.3. Conductivity event histogram of the blank samples with Gaussian fit (red line). Out of 2577 events, maxima can be found at $G_{1}=(0.03 \pm 0.02) \mathrm{nS}$ and $G_{2}=(0.26 \pm 0.05) \mathrm{nS}$ and $G_{3}=(0.54 \pm 0.17) \mathrm{nS}$. The measuring buffer contained $1 \mathrm{M} \mathrm{KCl}, 10 \mathrm{mM}$ HEPES, pH 7.5 and the bilayers were composed of DPhPC/Chol 9:1.

These results confirm the destabilizing influence of detergents on lipid membranes mentioned above.[100] In contrast to BLMs, solvent-free bilayers miss the stabilizing effect of organic solvents and are thus more readily permeabilized by detergents. But even in BLMs, detergents cause changes in the membrane resistance and potential upon incorporation.[143,144] This explains the offsets sometimes observed in BLM measurements, as well as in solventfree bilayers during this project. LDAO, in particular, is known to influence the membrane thickness: it incorporates into the bilayer, increases the surface area and therewith reduces the thickness, which was shown for DMPC liposomes.[145] This effect is an explanation for the increased permeability of solvent-free bilayers, enabling a transient ion flux which exhibits channel-like activity in the measurements. In the following experiments, conductivities especially in the range below $0.1 \mathrm{nS}$ have to be discussed carefully, as they are influenced by detergent effects. 


\subsubsection{Conductivity and open probability of PorB wild type}

PorB wild type (wt) from Neisseria meningitides (Nme) and Neisseria gonorrhea $(\mathrm{Ngo})$ is already widely investigated using planar patch-clamp on BLMs, but there is no data existing on solvent-free bilayers. In BLMs, conductivities of $G_{\mathrm{T}}=1.0-1.5 \mathrm{nS}$ were found for the PorB trimer, ${ }^{[49,50,146]}$ resulting in a monomer conductivity of $G_{\mathrm{M}}=0.3-0.5 \mathrm{nS}$. This facilitates the assignment of the conductivity states found in solvent-free membranes during this project.

After addition of PorB wt, regular steps in the conductivity trace could be observed (Figure 4.4), representing gating of the channel. Gating almost always appeared between the closed state $(G=0 \mathrm{nS})$ and only one open state, indicating that no transition between the different open states occurred. The steps of about $1.5 \mathrm{nS}$ conductivity visible in Figure 4.4, are assigned to a whole trimer.
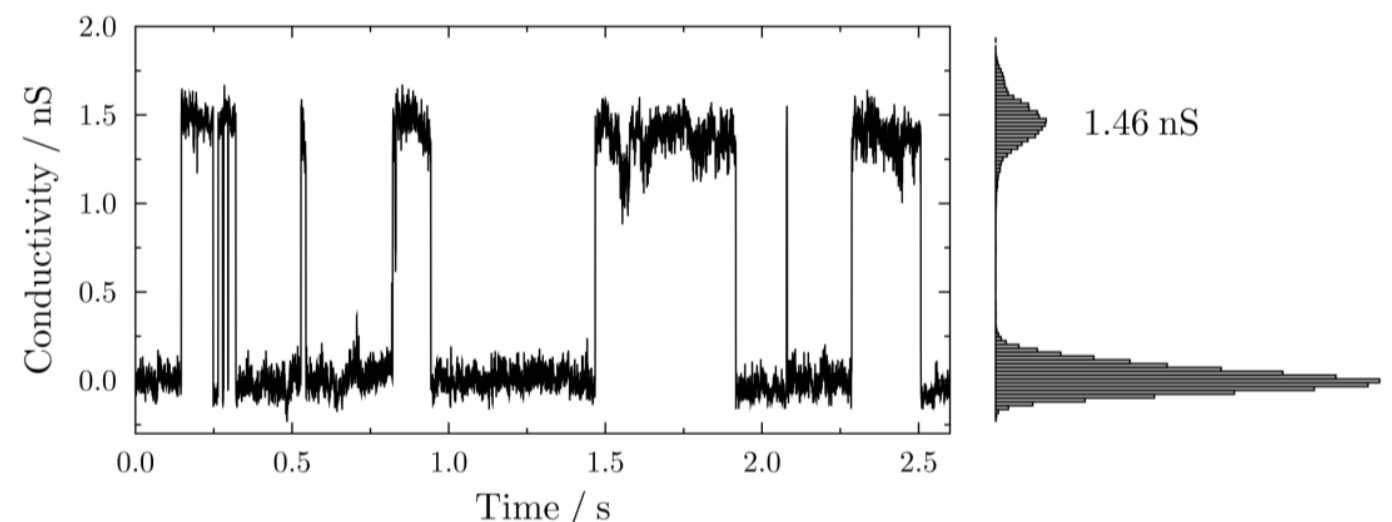

Figure 4.4. Conductivity trace and corresponding point amplitude histogram of PorB wt in a solvent-free membrane at an applied voltage of $+40 \mathrm{mV}$. The level of the open channel is about $1.5 \mathrm{nS}$, corresponding to a whole trimer. The measuring buffer contained $1 \mathrm{M} \mathrm{KCl}, 10 \mathrm{mM}$ HEPES, pH 7.5 and the bilayer was composed of DPhPC/Chol 9:1.

To determine the mean conductivities of monomeric, dimeric and trimeric state, a conductivity event histogram was generated from all measurements including data generated by Markus Schön during his master project. The fit of three Gaussian distributions to the data (5269 events) resulted in maxima at $G_{\mathrm{M}}=(0.39 \pm 0.18) \mathrm{nS}, G_{\mathrm{D}}=(0.84 \pm 0.26) \mathrm{nS}$ and $G_{\mathrm{T}}=(1.48 \pm 0.46) \mathrm{nS}$ (Figure 4.5), which can, according to literature,[49,50,146] be assigned to the monomeric, dimeric and trimeric state, respectively. The distribution of the events reflects the observations of the conductivity traces: If there were a lot of transitions between the three open states, the gating events of the monomer would be dominant, whereas only few trimer and dimer events should be visible. However, determination of the peak areas shows an equal distribution between trimer and dimer ( $40 \%$ each), while the monomer peak occupies only $20 \%$ of the total area. 


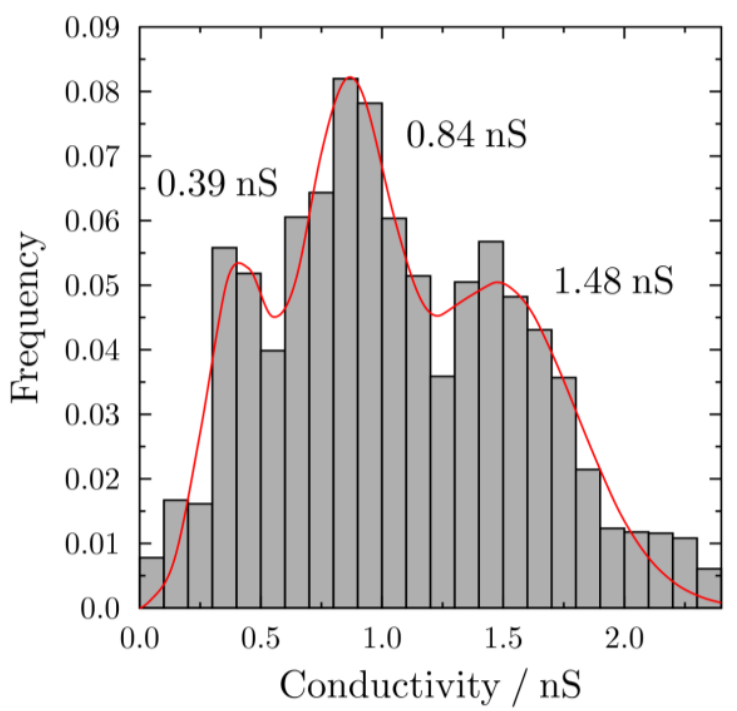

Figure 4.5. Conductivity event histogram of PorB wt in solvent-free membranes (5269 events) and Gaussian fit (red line). Three maxima at $G_{\mathrm{M}}=(0.39 \pm 0.18) \mathrm{nS}, G_{\mathrm{D}}=(0.84 \pm 0.26) \mathrm{nS}$ and $G_{\mathrm{T}}=(1.48 \pm 0.46) \mathrm{nS}$ are found. The measuring buffer contained $1 \mathrm{M} \mathrm{KCl}, 10 \mathrm{mM}$ HEPES, pH 7.5 and the bilayers were composed of DPhPC/Chol 9:1.

Open probabilities $\left(P_{0}\right)$ were determined separately for monomer, dimer and trimer, as in no case all three states were observed in one conductivity trace. The point amplitude histograms of all combined traces analyzed are depicted in Figure 4.6. For the conductivity of the monomer two states are found, both lying within the monomer-peak of the conductivity event histogram (compare Figure 4.5). The values are $G_{\mathrm{M}, 1}=(0.33 \pm 0.06) \mathrm{nS}$ and $G_{\mathrm{M}, 2}=(0.45 \pm 0.02) \mathrm{nS}(\mathrm{A})$. The open probability, resulting from the addition of the two maxima, was determined as $P_{\mathrm{o}, \mathrm{M}}=31 \%$. The probabilities of the dimer $\left(G_{\mathrm{D}}=(0.94 \pm 0.46) \mathrm{nS}, \mathrm{B}\right)$ and the trimer $\left(G_{\mathrm{T}}=(1.38 \pm 0.65) \mathrm{nS}, \mathrm{C}\right)$ are slightly lower with $P_{\mathrm{o}, \mathrm{D}}=21 \%$ and $P_{\mathrm{o}, \mathrm{T}}=21 \%$. In all cases, the closed state is the preferred one.
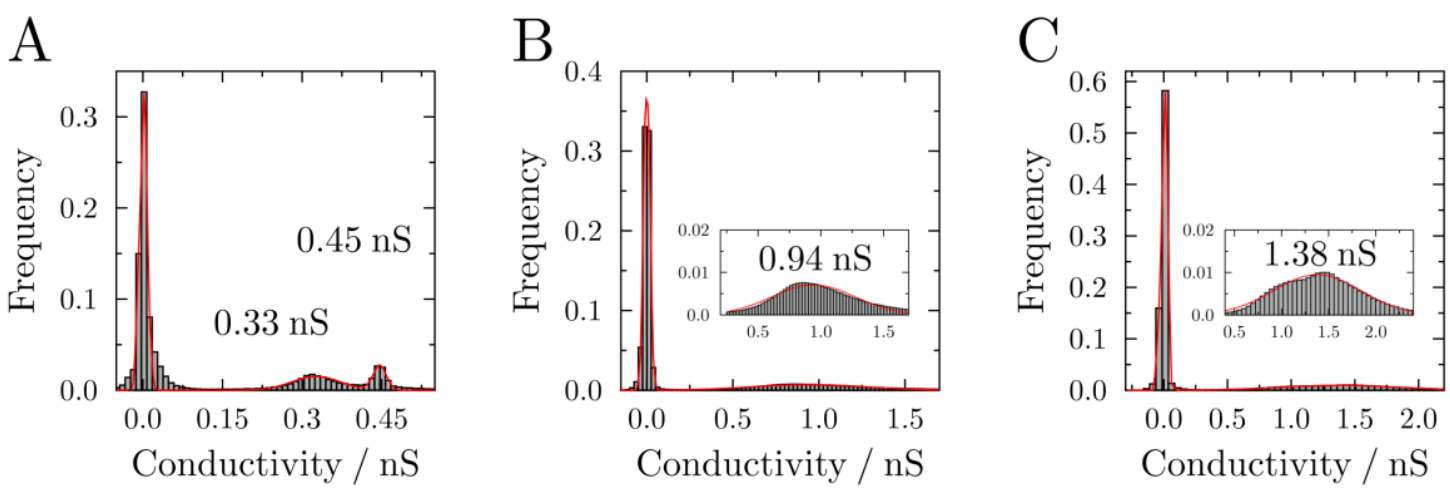

Figure 4.6. Point amplitude histograms and Gaussian fits (red lines) of the PorB wt monomer (A), dimer (B) and trimer (C). The determined conductivities are $G_{\mathrm{M}, 1}=(0.33 \pm 0.06) \mathrm{nS}$ and $G_{\mathrm{M}, 2}=(0.45 \pm 0.02) \mathrm{nS}$, $G_{\mathrm{D}}=(0.94 \pm 0.46) \mathrm{nS}$ (see insert of B) and $G_{\mathrm{T}}=(1.38 \pm 0.65) \mathrm{nS}$ (see insert of C). In all cases, the closed state is preferred. The measuring buffer contained $1 \mathrm{M} \mathrm{KCl}, 10 \mathrm{mM}$ HEPES, pH 7.5 and the bilayers were composed of DPhPC/Chol 9:1. 


\subsubsection{Conductivity and open probability of PorB G103K}

In the mutant PorB G103K, the glycine at position 103, located at the internal loop, is replaced by a lysine, introducing a larger residue and an additional positive charge. As the region of the ion permeation pathways overlaps with this position,[35] the mutation could have an influence on the conductivity of the pore, due to changed electrostatic interactions or an increased steric hindrance.

The mutant was thus electrophysiologically investigated under the same conditions as the wild type. After protein addition, gating of the channel was visible as steps in the conductivity trace (Figure 4.7). As for the wild type, a transition between different open states was almost never observed, but rather the transition between the closed state and only one open state.

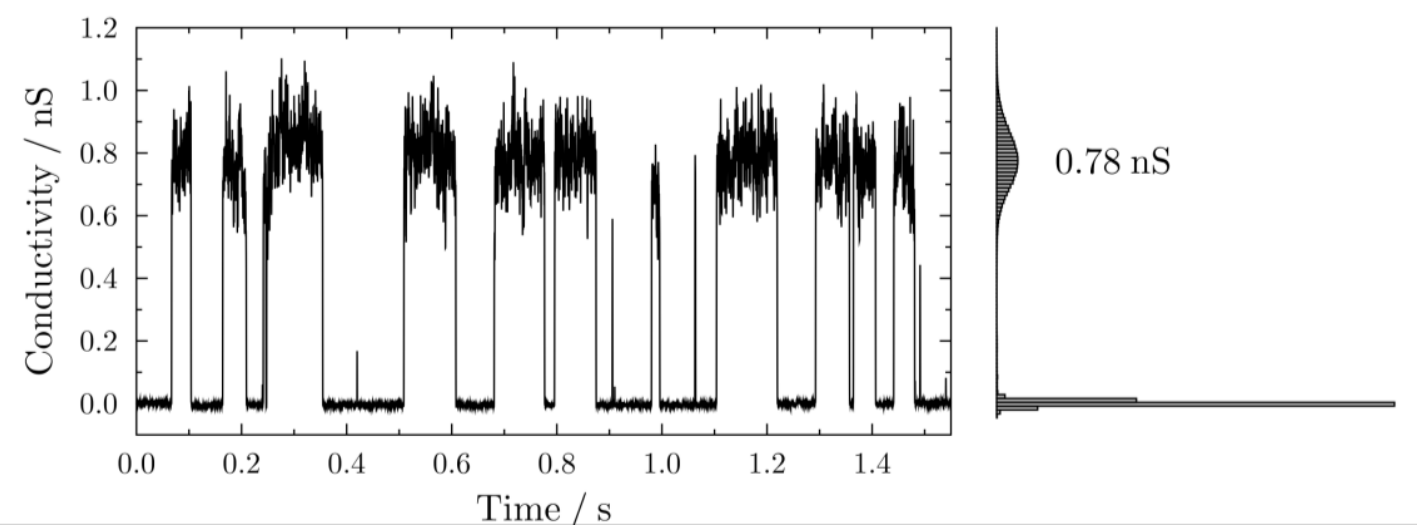

Figure 4.7. Conductivity trace of PorB G103K in solvent-free membranes recorded at $+40 \mathrm{mV}$ and corresponding point amplitude histogram. The measuring buffer contained $1 \mathrm{M} \mathrm{KCl}, 10 \mathrm{mM}$ HEPES, pH 7.5 and the bilayer was composed of DPhPC/Chol 9:1.

The conductivity event histogram was generated from 3461 gating events and three mean conductivities, $G_{\mathrm{M}}=(0.11 \pm 0.05) \mathrm{nS}, G_{\mathrm{D}}=(0.34 \pm 0.08) \mathrm{nS}$ and $G_{\mathrm{T}}=(0.73 \pm 0.14) \mathrm{nS}$, were determined by fitting three Gaussian distributions (Figure 4.8). The maximum at $0.11 \mathrm{nS}$ lies within a range, in which events were as well found in the blank samples (see Figure 4.3). Therefore, an influence of these events, caused by LDAO from the PorB buffer, on the monomer conductivity cannot be excluded. This would lead to a reduced mean conductivity value. Considering the values found for PorB wt, the maximum at $0.34 \mathrm{nS}$ could be assigned to the monomer, and the one at $0.73 \mathrm{nS}$ to the dimer. This would mean that the maximum at $0.11 \mathrm{nS}$ would be completely caused by LDAO and the trimer conductance is missing due to a damaged protein sample. This possibility was ruled out by the results from the no-SDS PAGE (see Chapter 4.1 and Figure 4.1), which showed the presence of trimers in different stock solutions of PorB G103K. Therefore, the value of $0.73 \mathrm{nS}$ is assigned to the trimer and the assignment of the other conductivities is correct. 
Here again, the monomer peak is not the one occupying the largest area, which would mean monomer activity was dominant. The areas are almost equally distributed between monomer (30\%), dimer (40\%) and trimer (30\%), implying they are almost equally active. Considering the overlap with the detergent-events, the area of the monomer is even smaller.

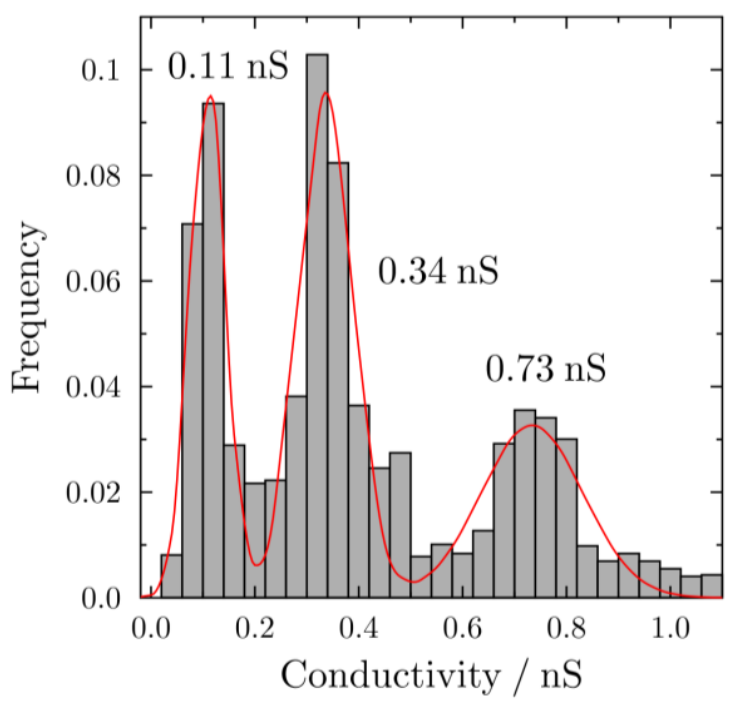

Figure 4.8. Conductivity event histogram of PorB G103K in solvent-free membranes ( 3461 events) and Gaussian fit (red line). The maxima were detected as $G_{\mathrm{M}}=(0.11 \pm 0.05) \mathrm{nS}, G_{\mathrm{D}}=(0.34 \pm 0.08) \mathrm{nS}$ and $G_{\mathrm{T}}=(0.73 \pm 0.14) \mathrm{nS}$. The measuring buffer contained $1 \mathrm{M} \mathrm{KCl}, 10 \mathrm{mM}$ HEPES, pH 7.5 and the bilayers were composed of DPhPC/Chol 9:1.

Open probabilities of monomer, dimer and trimer were again determined from separately recorded current traces. Data recorded by Hannah Maatsch during her Bachelor project contributed to these results. The obtained amplitude histograms of all traces combined are presented in Figure 4.9. The analysis reveals the lowest open probability for the monomer $\left(G_{\mathrm{M}, 1}=(0.13 \pm 0.03) \mathrm{nS}\right.$, and $G_{\mathrm{M}, 2}=(0.17 \pm 0.02) \mathrm{nS} \quad$ A) with $P_{\mathrm{o}, \mathrm{M}}=29 \%$. The trimer $\left(G_{\mathrm{T}}=(0.78 \pm 0.13) \mathrm{nS}, \mathrm{C}\right)$ shows a slightly higher probability of $P_{\mathrm{o}, \mathrm{T}}=39 \%$ and the highest probability of $P_{\mathrm{o}, \mathrm{D}}=72 \%$ is found for the dimer $\left(G_{\mathrm{D}}=(0.32 \pm 0.1) \mathrm{nS}, \mathrm{B}\right)$. The dimer of PorB G103K is the only state that has a higher probability than the closed state, whereas in case of monomer and trimer the closed state is dominant, as already detected for the wild type. 

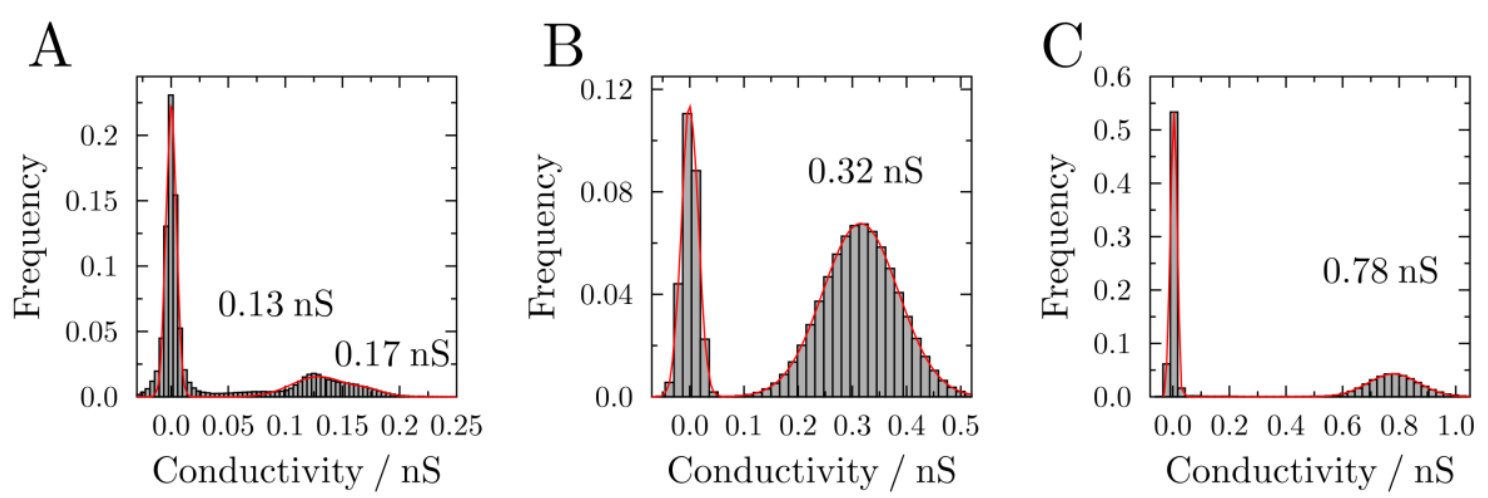

Figure 4.9. Point amplitude histograms and Gaussian fits (red lines) of the PorB G103K monomer (A), dimer (B) and trimer (C). The conductivities are found as $G_{\mathrm{M}, 1}=(0.13 \pm 0.03) \mathrm{nS}$ and $G_{\mathrm{M}, 2}=(0.17 \pm 0.02) \mathrm{nS}$, $G_{\mathrm{D}}=(0.32 \pm 0.1) \mathrm{nS}$ and $G_{\mathrm{T}}=(0.78 \pm 0.13) \mathrm{nS}$. Only in case of the dimer, the closed state is not the preferred one. The measuring buffer contained $1 \mathrm{M} \mathrm{KCl,} 10 \mathrm{mM}$ HEPES, pH 7.5 and the bilayers were composed of DPhPC/Chol 9:1.

\subsubsection{Conductivity and open probability of PorB G103D}

In the mutant G103D, the glycine at position 103 is substituted by aspartic acid, introducing a larger residue and an additional negative charge. The intention of characterizing this mutant in comparison with PorB G103K was to distinguish whether the nature of the charge and therewith the electrostatic interactions, or the steric hindrance of the residue has a stronger influence on the electrophysiological properties of the pore. All measurements of PorB G103D in solvent-free membranes were performed by Svenja Kiehn during her bachelor project.

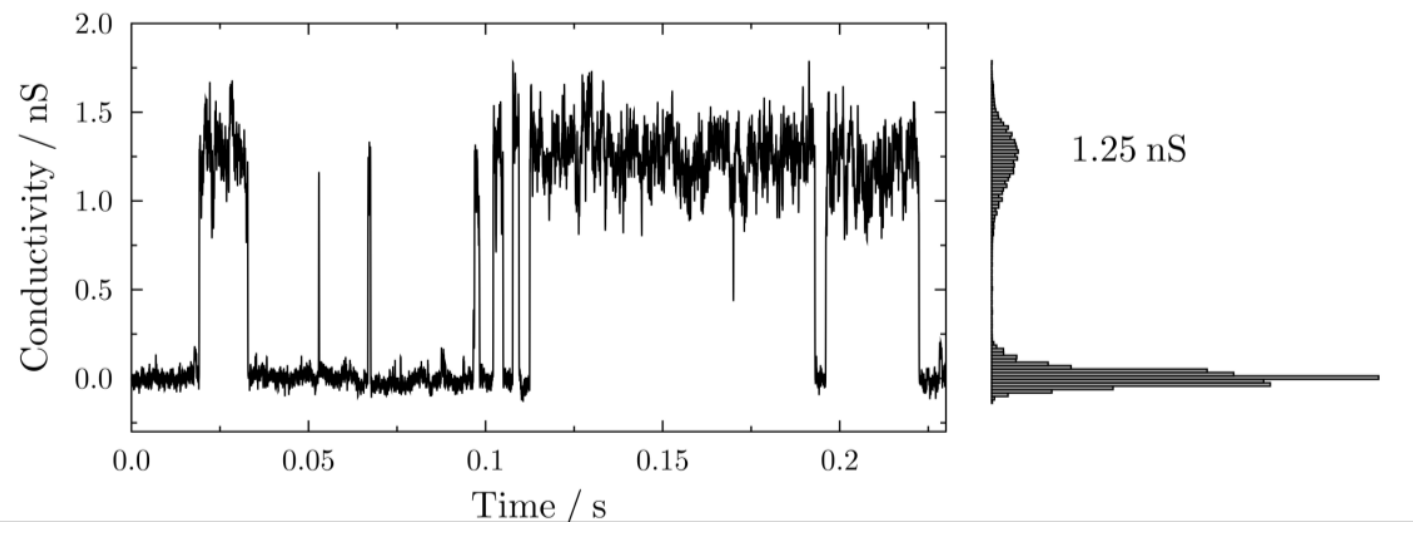

Figure 4.10. Conductivity trace of PorB G103D recorded at $+40 \mathrm{mV}$ in solvent-free membranes. The most frequently observed gating behavior of the pore is represented and the corresponding point amplitude histogram is shown on the right side. The measuring buffer contained $1 \mathrm{M} \mathrm{KCl}, 10 \mathrm{mM}$ HEPES, pH 7.5 and the bilayer was composed of DPhPC/Chol 9:1.

PorB G103D was investigated as described before and in most of the times, the steps in the conductivity traces showed the same behavior like the other two proteins: gating occurred between the closed state and one single open state (Figure 4.10). In one special case, however, unusually frequent gating was observed that appeared between the closed and three 
different open states, i.e., monomeric $(0.3 \mathrm{nS})$, dimeric $(0.82 \mathrm{nS})$ and trimeric $(1.15 \mathrm{nS})$ conductivity state (Figure 4.11).

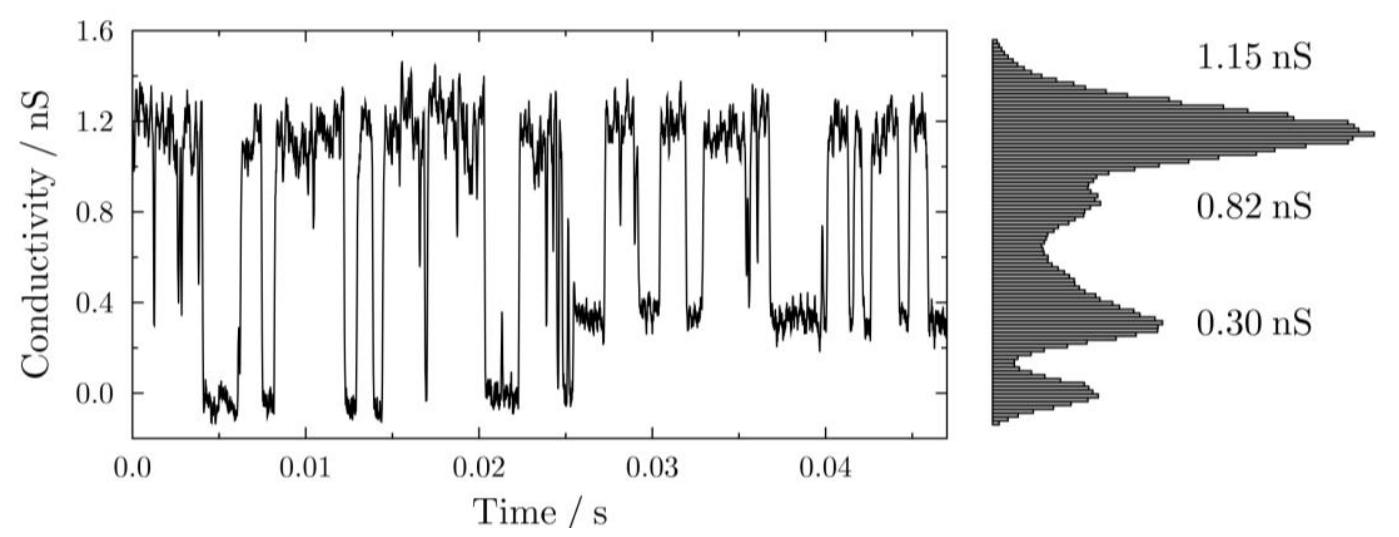

Figure 4.11. Unusual conductivity trace of PorB G103D recorded in solvent-free membranes at $+40 \mathrm{mV}$. Very frequent gating and transitions between all open states are visible. The measuring buffer contained $1 \mathrm{M} \mathrm{KCl}$, 10 mM HEPES, pH 7.5 and the bilayer was composed of DPhPC/Chol 9:1.

For the mean conductivities, 11094 gating events were analyzed, about 9000 of them arising from the one measurement with very frequent gating (Figure 4.11), as in all other measurements only few events were detected and the channel remained mostly in the closed state. The resulting conductivity event histogram with multiple Gaussian fits is shown in Figure 4.12. Two distinct maxima at $G_{\mathrm{T}}=(1.14 \pm 0.13) \mathrm{nS}$ and $G_{\mathrm{D}}=(0.73 \pm 0.19) \mathrm{nS}$ are found and assigned according to the data obtained for the wild type to the trimer and dimer conductivity, respectively. However, the values below $0.5 \mathrm{nS}$ are distributed very broadly, making a Gaussian fit infeasible. The maximum value was kept constant during the fitting routine in order to obtain the other two maxima as accurately as possible. 


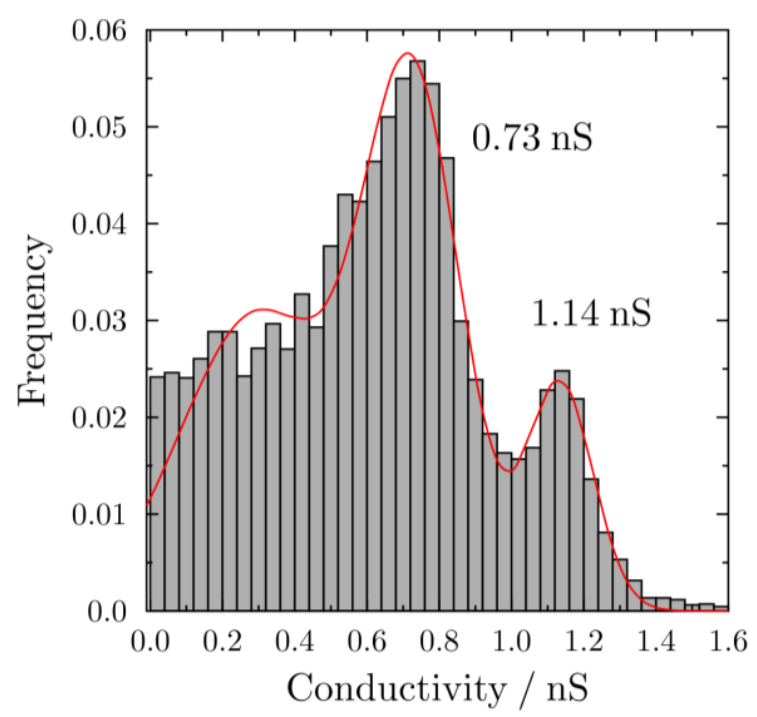

Figure 4.12. Conductivity event histogram of PorB G103D in solvent-free membranes (11094 events) and Gaussian fit (red line). Two maxima were determined as $G_{\mathrm{T}}=(1.14 \pm 0.13) \mathrm{nS}, G_{\mathrm{D}}=(0.73 \pm 0.19) \mathrm{nS}$, while the values below $0.5 \mathrm{nS}$ are distributed too broadly for a reliable fit. The measuring buffer contained $1 \mathrm{M} \mathrm{KCl,} 10 \mathrm{mM}$ HEPES, pH 7.5 and the bilayers were composed of DPhPC/Chol 9:1.

The analysis of the point amplitude histogram belonging to the trace with very frequent gating enabled to determine the conductivities of monomer, dimer and trimer in one dataset (Figure 4.13) using five Gaussian distributions (one for the shoulder at about $0.5 \mathrm{nS}$ ). For trimer and dimer, values very similar to the ones obtained from the conductivity event histogram are obtained with $G_{\mathrm{T}}=(1.15 \pm 0.16) \mathrm{nS}$ and $G_{\mathrm{D}}=(0.82 \pm 0.12) \mathrm{nS}$. For the monomer a conductivity of $G_{\mathrm{M}}=(0.30 \pm 0.09) \mathrm{nS}$ is determined. The open probabilities show a clear preference for the trimer state, which has a probability of $P_{\mathrm{o}, \mathrm{T}}=51 \%$, whereas dimer, monomer and the closed state occur with probabilities of $P_{\mathrm{o}, \mathrm{D}}=16 \%, P_{\mathrm{o}, \mathrm{M}}=25 \%$ and $P_{\mathrm{o}, \mathrm{C}}=8 \%$. In this special case, the closed state is not represented as the most abundant one. 


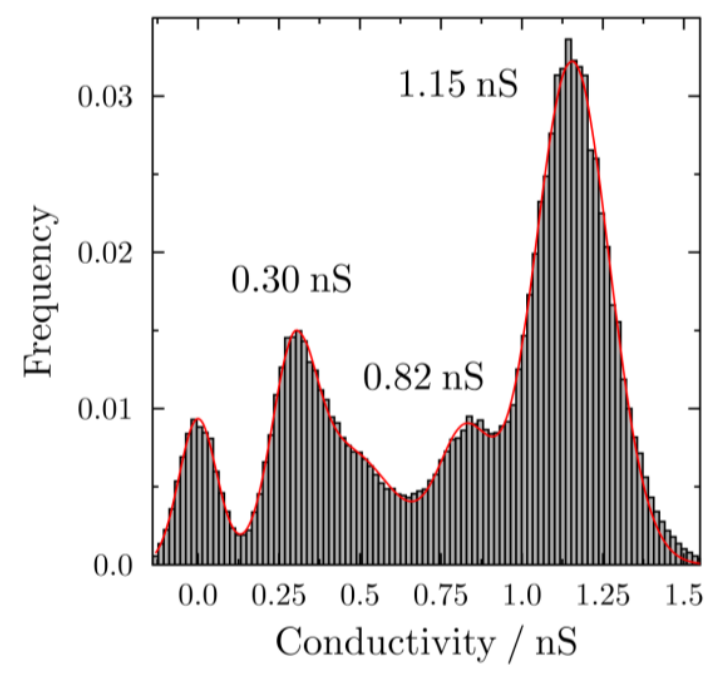

Figure 4.13. Point amplitude histogram of the conductivity trace showing frequent gating of PorB G103D. The transition between closed, monomeric, dimeric and trimeric state are represented by the determined maxima. The conductivities of the three open states are $G_{\mathrm{M}}=(0.30 \pm 0.09) \mathrm{nS}, G_{\mathrm{D}}=(0.82 \pm 0.12) \mathrm{nS}$ and $G_{\mathrm{T}}=(1.15 \pm 0.16) \mathrm{nS}$. The measuring buffer contained $1 \mathrm{M} \mathrm{KCl}, 10 \mathrm{mM}$ HEPES, $\mathrm{pH} 7.5$ and the bilayer was composed of DPhPC.

In addition, conductivity traces representing the situation found in the majority of the measurements were investigated. Separate datasets were analyzed for monomer, dimer and trimer, showing a preference for the closed state in all cases. The evaluated point amplitude histograms of all combined conductivity traces are presented in Figure 4.14. A conductivity of $G_{\mathrm{M}}=(0.33 \pm 0.11) \mathrm{nS}$ and an open probability of $P_{\mathrm{o}, \mathrm{M}}=13 \%$ are obtained for the monomer (A). The dimer $\left(G_{\mathrm{D}}=(0.72 \pm 0.27) \mathrm{nS}, \mathrm{B}\right)$ and the trimer $\left(G_{\mathrm{T}}=(1.17 \pm 0.23) \mathrm{nS}, \mathrm{C}\right)$ have similar probabilities of $P_{\mathrm{o}, \mathrm{D}}=14 \%$ and $P_{\mathrm{o}, \mathrm{T}}=18 \%$. Thus, the closed state of PorB G103D is clearly preferred compared to the open state.
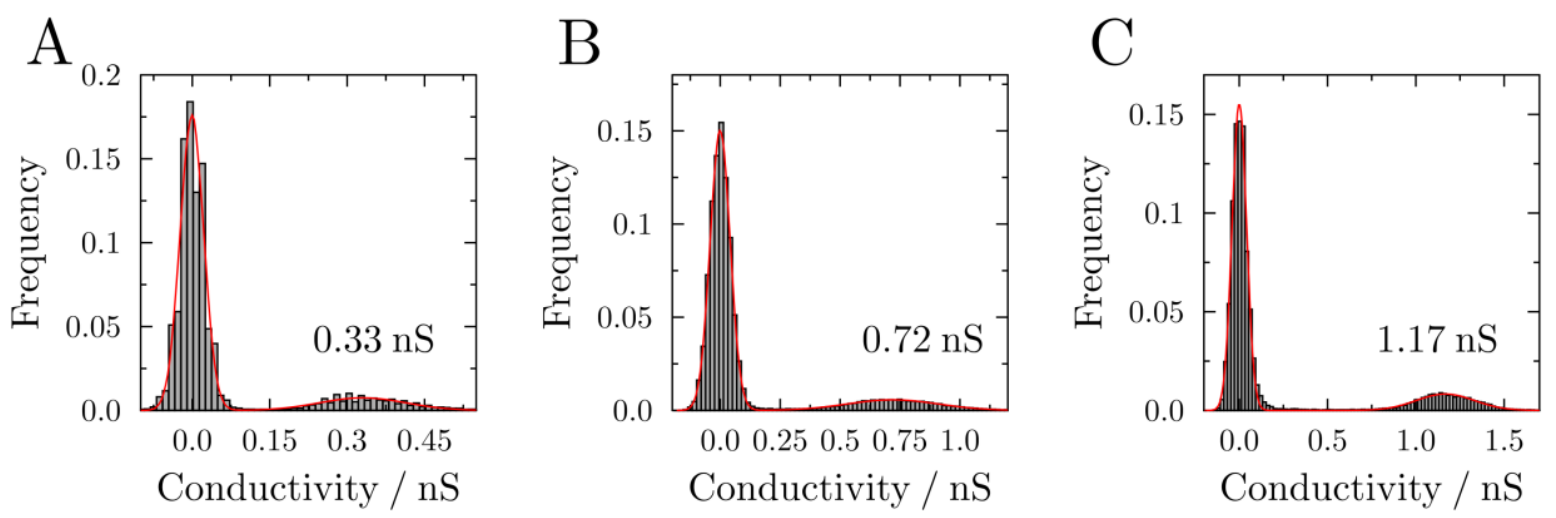

Figure 4.14. Amplitude histograms and Gaussian fits (red lines) of the PorB G103D monomer (A), dimer (B) and trimer $(\mathrm{C})$. The conductivities are $G_{\mathrm{M}}=(0.33 \pm 0.11) \mathrm{nS}, G_{\mathrm{D}}=(0.72 \pm 0.27) \mathrm{nS}$ and $G_{\mathrm{T}}=(1.17 \pm 0.23) \mathrm{nS}$. The closed state is clearly preferred with probabilities of over $80 \%$. The measuring buffer contained $1 \mathrm{M} \mathrm{KCl}, 10 \mathrm{mM}$ HEPES, pH 7.5 and the bilayers were composed of DPhPC/Chol 9:1 or pure DPhPC. 


\subsubsection{Comparison of PorB wild type and the mutants in solvent-free mem- branes}

\section{Channel conductance}

The reduction of the channel conductance due to the mutation is more pronounced for PorB G103K than for PorB G103D, indicating that the nature of the charge is the major cause.

Mean conductivities of monomeric, dimeric and trimeric state of the three proteins are summarized in Table 4.1. The values are taken from the conductivity event histograms, except for the monomer of PorB G103D, which could only be obtained from the point amplitude histogram (Figure 4.13), because the maximum in the conductivity event histogram could not be determined correctly (compare Figure 4.12). Monomer and dimer conductivities for the wild type and G103D are similar, but slightly lower for the mutant. Comparing the trimer conductivities, the one of the wild type is notably higher. For G103K, however, notably lower conductivities were determined for all three states. The possibility of a missing trimer conductance due to monomer dissociation in the stock solution is unlikely due to the results of the no-SDS PAGE (see Chapter 4.1). Of note, for none of the proteins, the values of one monomer multiplied by three leads to the determined trimer conductivity. In case of PorB G103D, the monomer value was only obtained from the point amplitude histogram of one conductivity trace and could thus have a larger error range than indicated by the FWHM. A trimer conductivity of $1.14 \mathrm{nS}$ would imply a monomer conductivity of about $0.38 \mathrm{nS}$ and a dimer conductivity of about $0.76 \mathrm{nS}$, which is very close to the determined value. In case of PorB G103K, the monomer value is partially influenced by the detergent effects (see Chapter 4.2.1.1), leading to a lower conductance. Nonetheless, dimer and trimer value do not match. In case of PorB wt, the monomer value is about half of the dimer value, but the trimer value is too high. This could be due to the large variation of the measurements as reflected by the broad distribution of the maximum.

Table 4.1. Conductivities of PorB wt, G103K and G103D in solvent-free membranes. Values are obtained from the conductivity event histograms, except for the PorB G103D monomer, which is taken from the point amplitude histogram in Figure $4.13^{(*)}$. Errors are the FWHM.

\begin{tabular}{c|ccc}
\hline $\boldsymbol{G} / \mathbf{n S}$ & PorB wt & PorB G103K & PorB G103D \\
\hline Monomer & $0.39 \pm 0.18$ & $0.11 \pm 0.05$ & $0.30 \pm 0.09\left(^{*}\right)$ \\
Dimer & $0.84 \pm 0.26$ & $0.34 \pm 0.07$ & $0.73 \pm 0.19$ \\
Trimer & $1.48 \pm 0.46$ & $0.73 \pm 0.14$ & $1.14 \pm 0.13$ \\
\hline
\end{tabular}


Reduced channel conductivities upon point mutations at loop 3 have been reported for porins before. The mutation G119D in OmpF resulted in a separation of the constriction zone into two compartments and a conductance decrease by one third.[147] The OmpF-like protein MOMP showed a 70 \% lower conductance due to the mutation G112D.[148] Olesky et al. investigated PorB wild type from Neisseria gonorrhea and several mutants using black lipid membranes. Among them was the point mutation G120K, which corresponds to the mutation G103K in PorB from Neisseria meningitidis ${ }^{[149]}$ and the mutant G120D/A121D, where the mutation equivalent to G103D is accompanied by a second substitution by an aspartic acid. The predominant conducting state of both mutants was 30-40\% lower than the one of the wild type.[49]

The reduced conductance of G103K is in good agreement with these findings and can be explained by molecular dynamics (MD) simulations of the ion flux through PorB (Nme) wild type and G103K. Strongly separated pathways for anion and cation translocation were detected in the wild type.[62,140] In the mutant, however, the cation flux was found to be nearly completely interrupted.[140] This extreme reduction of the cation permeation is a reasonable explanation for an overall reduced conductance.

The analysis of the electrostatic surface potential performed by Mikio Tanabe (Institute of Materials Structure Science, Structural Biology Research Center, Ibaraki, Japan, unpublished results) of both porins suggests that the additional positive charge of G103K causes this interruption of the cation pathway (Figure 4.15). At the periplasmic side the positive potential present in the wild type (A) disappears and leaves a circle of positive potential around the constriction region in the mutant (B). At the extracellular side, the positive surface potential is as well increased for the mutant (D) compared to the wild type (C). Thus, the impaired cation pathway is probably caused by electrostatic repulsions at the constriction zone. 

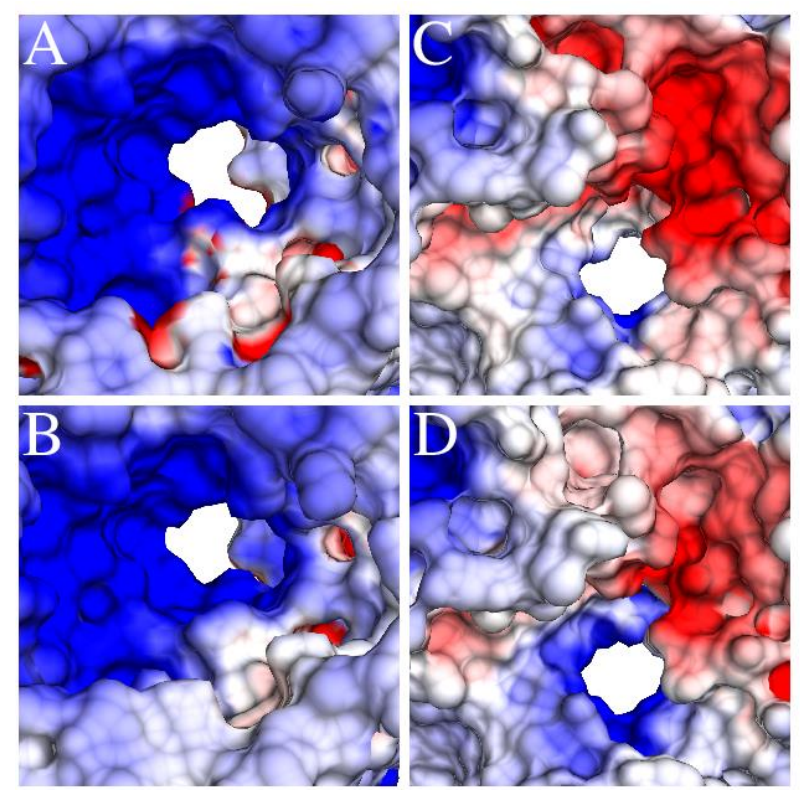

Figure 4.15. Electrostatic surface potential of PorB wt (A, C) and G103K (B, D). View of the periplasmic (A, B) and extracellular (C,D) side. The density of the positively charged $\varepsilon$-amino group in Lys103 of PorB G103K was not well ordered. Therefore, a model with 9 possible positions of the $\varepsilon$-amino group was constructed and the electrostatic surface potential was calculated for each structure. All structures showed very similar surface potential distributions and one example is shown in (B) and (D). Potentials are contoured from $+8 k T /$ e to $-8 k T / e$, positive potential is depicted in blue, negative potential in red. (Mikio Tanabe, Institute of Materials Structure Science, Structural Biology Research Center, Ibaraki, Japan, unpublished results)

Surprisingly, in this study a distinct reduction of the conductivity of the mutant PorB (Nme) G103D was only found for the trimeric state. For other porins a comparable mutation is described to alter the conducting properties. ${ }^{[147,148]}$ In PorB (Ngo) the corresponding mutation G120D was investigated together with a second point mutation (A121D). Thus, it cannot be distinguished which mutation contributed to which extent to the decrease of the channel conductance, or whether the combination of both is necessary for this effect. Unfortunately, no MD simulations of the ion flux exist for PorB G103D. However, preliminary results of the surface potential calculation revealed only a slight increase of the negative areas compared to the wild type (Mikio Tanabe, Institute of Materials Structure Science, Structural Biology Research Center, Ibaraki, Japan, unpublished results). Therefore, probably the anion flux is slightly altered, but apparently not as much as the cation flux in the G103K mutant.

These results indicate that the sign of the charge introduced at position 103 is indeed crucial for the level of the channel conductivity, as an additional positive charge influences the pore conductance more extensively than a negative one. Another aspect is the length of the side chain, which contains more $\mathrm{CH}_{2}$-units between the charge and the amino acid moiety for lysine than for aspartic acid. Consequently, the positive amino group can probably protrude further into the pore than the negative carboxyl group. The constriction area is reduced by approximately 3-5 $\AA$ due to the mutation G->K, but no estimation exists for the mutation 
G->D (Mikio Tanabe, Institute of Materials Structure Science, Structural Biology Research Center, Ibaraki, Japan, personal communication).

\section{Gating behavior}

For all three proteins, gating almost never occurred between monomer, dimer and trimer open state, which hints at a high level of cooperativity between the single monomers.

PorB wild type and the mutant G103K almost exclusively showed gating between the closed state and only one open state, whereas gating between the individual open states representing PorB monomer dimer or trimer almost never occurred. Most of the time, the mutant G103D presented the same behavior as well, but in one measurement exceptionally frequent gating and the transition between all open states was visible (see Figure 4.11).

Simultaneous opening and closing of the PorB monomers indicates gating cooperativity. The transition of one monomer from e.g., closed to open state might cause a conformational change in the other two forcing them into the open conformation as well, on a time-scale too fast for the time resolution of the electrophysiological measurements. A certain level of cooperativity was detected for PorB wt (Nme) before. ${ }^{[50]}$ Almost simultaneous closing of two or all three monomers was observed, the first occurring more frequent than the latter. For OmpF and OmpC the interaction between the monomers depends on the $\mathrm{pH}$ value and the applied voltage. ${ }^{[150,151]}$ At low $\mathrm{pH}$ and high voltages the level of cooperativity decreases more and more. At pH $5.5 \mathrm{OmpF}$ shows a cooperative behavior up to $100 \mathrm{mV}$, as only the trimer state is detected, while at $125 \mathrm{mV}$ monomer and dimer activity is found as well. At $\mathrm{pH} 3.5$, however, already at $25 \mathrm{mV}$ monomer and dimer gating occurs and at $75 \mathrm{mV}$ the monomer is more active than dimer and trimer. ${ }^{[150]} \mathrm{A}$ less tight association of the monomers is discussed, possibly reducing the influence of the monomers on each other. In $\mathrm{OmpF}$, the association of the monomers is mainly achieved by hydrophobic interactions between the $\beta$-barrels, complemented by a connection realized by loop L2, which is partially integrated into the channel of one neighboring monomer. This interaction between L2 and the neighboring $\beta$-barrel is based on salt bridges and hydrogen bonds,[152] explaining the influence of the $\mathrm{pH}$. Due to the high structural analogy of OmpF and PorB (Nme), ${ }^{[35]}$ a similar situation can be assumed for the association of PorB monomers. Indeed, the crystal structure of PorB wt (Nme) (PDB-ID: 3VZT) [76] reveals that $\mathrm{L} 2$ of one monomer protrudes into the $\beta$-barrel of another (Figure 4.16). 


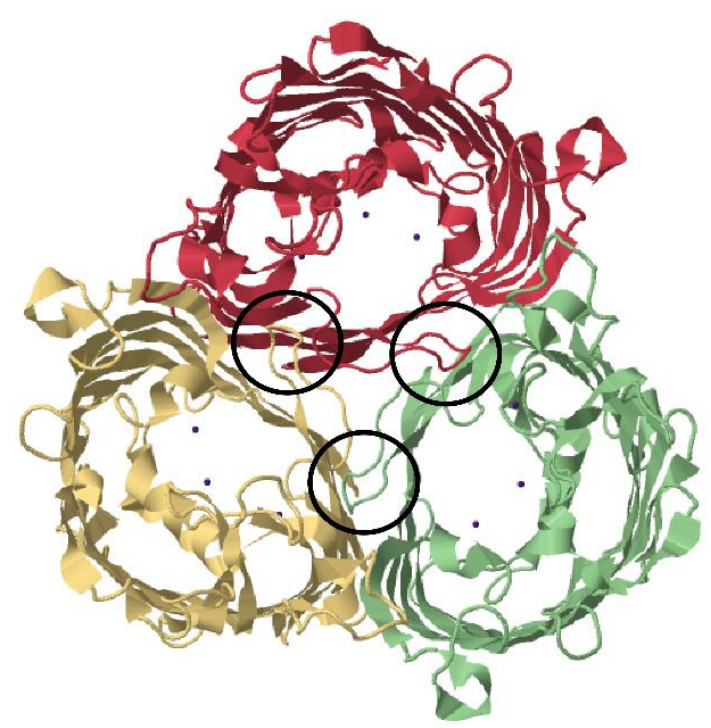

Figure 4.16. Trimeric structure of PorB wt (Nme) with different coloring of the monomers. The areas where loop L2 of one monomer interacts with the $\beta$-barrel of another one are marked with black circles. Modified from RCSB PDB (PDB-ID: 3VZT).[76]

With pH 7.5 and an applied voltage of $40 \mathrm{mV}$, the experimental conditions used in this project for the measurements on solvent-free bilayers fulfill the criteria necessary for a cooperativity of the OmpF monomers. Thus, the cooperative gating observed for PorB wt and G103K is in good agreement with those findings. The cooperative gating behavior detected for PorB wild type (Nme) by Song and coworkers ${ }^{[50]}$ was not investigated dependent on the applied voltage (ranging from -120 to $+120 \mathrm{mV}$ ) or $\mathrm{pH}$ (not indicated).

Due to the one measurement of PorB G103D that showed the transition between open monomer, dimer and trimer, one could assume a lower level of cooperativity for this mutant than for the other two porins. However, considering that this more independent gating of the single monomers was only observed in one measurement, together with for PorB G103D unusually frequent gating, no conclusion concerning the influence of this mutation on the gating cooperativity of the monomers is possible from the experiments on solvent-free membranes. This observation would have been ascribed to a damaged protein sample, if the same behavior had not occurred in the measurements using black lipid membranes (for further discussion see Chapter 4.2.2.5).

\section{Open probabilities}

The closed state of PorB is predominant in solvent-free bilayers. The deviating tendency of PorB G103K is ascribed to changes of the inner electric field due to the mutation. 
Open probabilities of PorB wt and both mutants are presented in Table 4.2. Values are taken from the point amplitude histograms analyzed separately for the three states (monomer, dimer and trimer). For PorB wt and G103D, a preference of the closed state over the open one was found for all three states. Except for the monomer of PorB wt, both proteins show similar probabilities. G103K, however, exhibits higher open probabilities, especially for the dimer, which is the only one showing a predominance of the open state. As the different measurements presented large variations of the determined open probabilities, the comparison of the absolute values is not suitable and only general tendencies are discussed.

Table 4.2. Open probabilities of PorB wt, G103K and G103D in solvent-free membranes. The values are taken from the point amplitude histograms generated separately for monomer, dimer and trimer.

\begin{tabular}{c|ccc}
\hline \multicolumn{1}{c}{$\mathbf{P}_{\mathbf{o}} / \boldsymbol{\%}$} & PorB wt & PorB G103K & PorB G103D \\
\hline Monomer & 31 & 29 & 13 \\
Dimer & 21 & 72 & 14 \\
Trimer & 21 & 39 & 18 \\
\hline
\end{tabular}

During electrophysiological investigations of PorB ( $\mathrm{Ngo}$ ) wild type and mutants corresponding to G103K and G103D in PorB (Nme), the mutants turned out to spend more time in subconductance states, thus exhibiting lower open probabilities than the wild type.[49] Certain mutations at the internal loop of OmpU from Vibrio choleraea present a decreased open probability, caused by an increased gating frequency, whereas others show no change compared to the wild type.[153] In OmpC, some mutations located at different positions of loop3 also led to lower open probabilities due to the increased frequency of gating compared to the wild type, which only rarely left the open state.[154,155] For positions similar to 103 in PorB Nme, changed electrostatic interactions between charged residues are discussed as most probable reason.[155] Negatively charged residues located at loop3 face a cluster of positively charged ones at the barrel wall. This way they form an electrical field within the constriction zone, which is presumably altered by the investigated mutations. For PorB wt (Nme) the situation is similar: negatively charged amino acids are gathered at the internal loop, whereas at the opposed barrel wall positive charges are dominant.[149] The electrical field is thus influenced by the introduction of a positive charge (G103K) to loop3, but not that much by an additional negative one at the same position (G103D), as indicated by the calculation of the electrostatic surface potential, which is only altered by the mutation G103K (see Figure 4.15). An influence of the electric field could explain the altered open probabilities found 
for PorB G103K compared to the other two PorB variants, although no differences in the gating frequency were observed.

\subsubsection{Results of black lipid membrane (BLM) measurements}

In order to compare the electrophysiological properties of PorB wild type and the mutants obtained in solvent-free membranes (Port-a-Patch, see Chapter 4.2.1) with solvent containing membranes, the proteins were investigated in black lipid membranes (BLMs). Bilayers were formed by painting of a lipid/decane mixture over an aperture $(d=50 \mu \mathrm{m})$ in a Teflon foil (BLM set-up, see Chapter 3.6.3.2) or a MECA (Micro Electrode Cavity Array, Ionera, Freiburg) Chip (Orbit 16, see Chapter 3.6.3.3). For the BLM set-up, bilayers were composed of $\mathrm{DPhPC} / \mathrm{Chol}$ 9:1, while for the Orbit 16 pure DPhPC bilayers were formed. The presence of $10 \%$ cholesterol did not change the protein's electrophysiological properties. Hence, the recordings were evaluated in the same analysis. The protein was inserted at $+40 \mathrm{mV}$ (addition to cis side) and the measurements were recorded at $\pm 40, \pm 60, \pm 80$ and $\pm 100 \mathrm{mV}$. For measurements using the self-constructed BLM-setup, a sampling rate of $50 \mathrm{kHz}$ and a low pass filter frequency of $5 \mathrm{kHz}$ was applied. The recordings using the Orbit 16 and the MECA chips were performed at a chosen filter frequency of $5 \mathrm{kHz}$, obtained by selecting a sampling rate of $10 \mathrm{kHz}$ (see Chapter 3.6.3.3).

During the blank measurements adding only pure PorB buffer $(200 \mathrm{mM} \mathrm{NaCl}, 20 \mathrm{mM}$ Tris, $0.1 \%(w / w)$ LDAO $)$ without protein no gating-like events or even disturbances of the bilayer were observed, thus no conductivity trace or event histogram is presented. It can thus be assumed that the detergent present in the PorB stock solutions has no influence on the stability of the BLMs.

\subsubsection{Conductivity and open probability of PorB wild type}

The first important observation after addition of PorB wt to a BLM is the clear preference for the open state. Most of the insertions resulted in channels completely open for long time periods interrupted only by occasional gating events. The amount of events increased with increasing positive voltage. Some exceptions were observed where an insertion was followed by several hours of continuous, very frequent gating. From these measurements, the channel conductivities were determined. A section of such a conductivity trace recorded at $+40 \mathrm{mV}$ is presented in Figure 4.17. In addition to the mainly occurring steps between 1.28 and $0.34 \mathrm{nS}$, steps between 0.34 and $0 \mathrm{nS}$ were occasionally visible. The corresponding point amplitude 
histogram shows a clear preference for the open state while the states at 0.34 and $0 \mathrm{nS}$ occur only rarely.

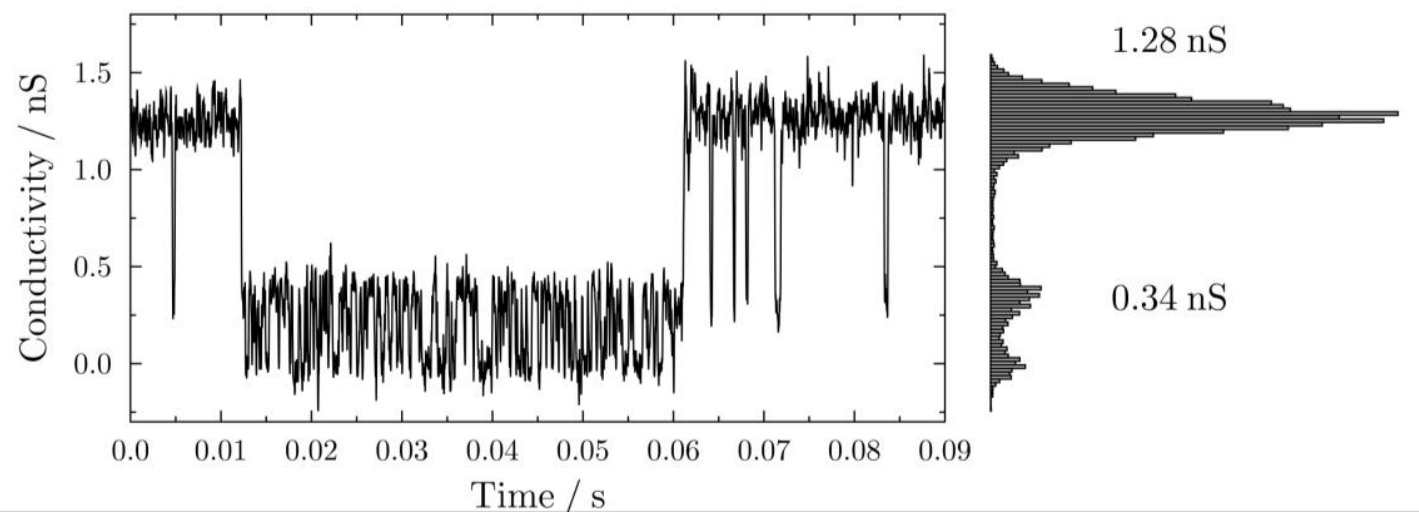

Figure 4.17. Section of a conductivity trace of PorB wt in a BLM recorded at $+40 \mathrm{mV}$ and corresponding point amplitude histogram. In this exceptional case, very frequent gating was observed. The measuring buffer contained $1 \mathrm{M} \mathrm{KCl}, 10 \mathrm{mM}$ HEPES, pH 7.5 and the bilayer was composed of DPhPC/Chol 9:1.

In view of the huge amount of gating events in a short period, only some sections were analyzed for the conductivity event histogram, altogether 7269 events. Because the applied voltage turned out to influence the conductivity (see results below and Figure 4.19 B), only values obtained at $40 \mathrm{mV}$ were evaluated to ensure a direct comparability with the solvent-free membranes. Only one maximum at $G=(0.95 \pm 0.07 \mathrm{nS})$ was found (Figure 4.18), the gating between 0 and $0.34 \mathrm{nS}$ visible in the current trace (see Figure 4.17) occurred so rarely that it is not represented in the conductivity histogram. According to the results obtained in solventfree membranes (see Figure 4.5), this maximum is assigned to the dimer conductivity. Looking at the conductivity trace (Figure 4.17), the rarely occurring state at $0.34 \mathrm{nS}$ represents the monomer conductivity and the dominant state at $1.28 \mathrm{nS}$ belongs to the fully open trimer. The observed dimer gating solely occurred between the open monomer and the open trimer state. 


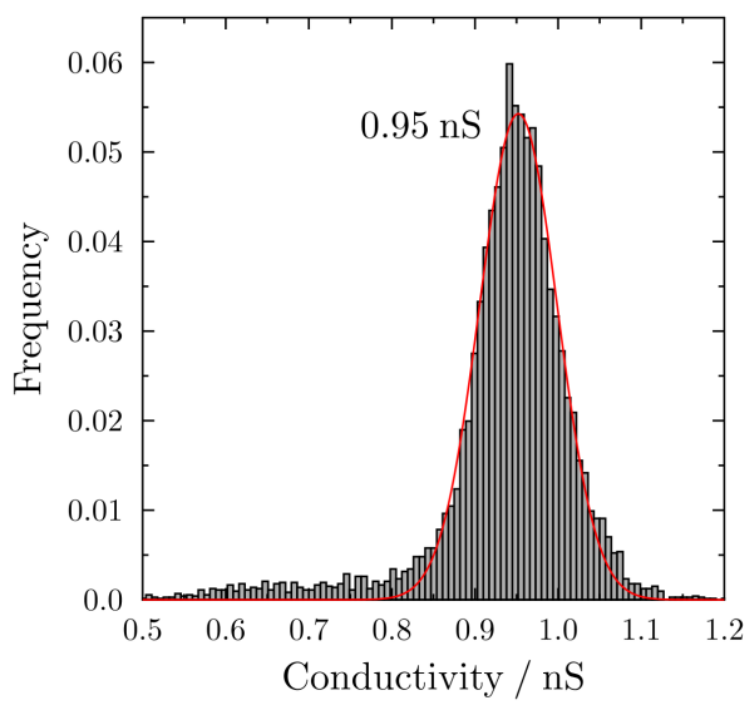

Figure 4.18. Conductivity event histogram of PorB wt recorded in BLMs at $+40 \mathrm{mV}$ and Gaussian fit (red line). 7269 events were analyzed and a conductivity of $G=(0.95 \pm 0.07 \mathrm{nS})$ is determined. The measuring buffer contained $1 \mathrm{M} \mathrm{KCl}, 10 \mathrm{mM}$ HEPES, pH 7.5 and the bilayers were composed of DPhPC/Chol 9:1.

Conductivity traces with very frequent gating were recorded at $\pm 40, \pm 60, \pm 80$ and $\pm 100 \mathrm{mV}$, enabling a determination of the open probability and - in the same analysis of the point amplitude histograms - as well the conductivity dependent on the applied voltage. In contrast to the experiments in solvent-free membranes, only one open state could be analyzed. Thereby it has to be noted, that the determined open probabilities belong to the completely open trimer, but the simultaneously calculated conductivities are the ones of the open dimer, as it is the one showing the gating activity (compare Figure 4.17) and the state at $0 \mathrm{nS}$ occurred so rarely that it did not show up in the point amplitude histograms. Consequently, for the determination of the conductivities, the differences between open monomer state and open trimer state is represented in the histogram. The values were obtained by averaging the values of all recorded conductivity traces. The errors are the standard deviation, for the open probability and the conductivity (see Chapter 3.6.4.1). As all traces were recorded in one experiment, the standard deviation is very small and had to be rounded up.

In Figure $4.19 \mathrm{~A}$, an exemplary point amplitude histogram of data recorded at $+40 \mathrm{mV}$ is displayed. For the other voltages, the histograms are very similar: Only few values were found for the open monomer state and most of the time the trimer was completely open. The conductivity value at $-100 \mathrm{mV}$ could not be determined, because there were not enough data points for the monomer state to fit a Gaussian distribution. Assuming a probability of $0 \%$ for the state of only one open monomer, an open probability for the trimer at - $100 \mathrm{mV}$ could be determined, nonetheless. The deviation from $100 \%$ open probability is caused by a second 
open state around $0.6 \mathrm{nS}$, found sometimes at negative applied voltages. It might be caused by increased membrane fluctuations due to the high voltage.

Figure 4.19 B shows the conductivities of PorB wt determined from the point amplitude histograms at different voltages. With increasing voltage the conductivity is reduced, from $G_{\mathrm{D},+40 \mathrm{mV}}=(0.94 \pm 0.01) \mathrm{nS}$ at $+40 \mathrm{mV}$ to $G_{\mathrm{D},+100 \mathrm{mV}}=(0.83 \pm 0.01) \mathrm{nS}$ at $+100 \mathrm{mV}$. At negative voltages a reversed tendency can be found, the conductivity is increased from $G_{\mathrm{D},-40 \mathrm{mV}}=(0.84 \mathrm{nS} \pm 0.01)$ at $-40 \mathrm{mV}$ to $G_{\mathrm{D},-80 \mathrm{mV}}=(0.90 \pm 0.01) \mathrm{nS}$ at $-80 \mathrm{mV}$.

In Figure 4.19 C, the open probability is plotted dependent on the applied voltage. For negative voltages only a very weak increase can be observed with decreasing voltage from $P_{\mathrm{o}, \mathrm{T},-40 \mathrm{mV}}=98 \%$ at $-40 \mathrm{mV}$ to $P_{\mathrm{o}, \mathrm{T},-100 \mathrm{mV}}=96 \%$ at $-100 \mathrm{mV}$. At positive voltages, however, the open probability shows a clear voltage dependence, decreasing with increasing voltage from $P_{\mathrm{o}, \mathrm{T},+40 \mathrm{mV}}=96 \%$ at $+40 \mathrm{mV}$ to $P_{\mathrm{o}, \mathrm{T},+100 \mathrm{mV}}=89 \%$ at $100 \mathrm{mV}$. Thus, the open probability of PorB wt in BLMs is higher at negative voltages and the open state is clearly preferred, in contrast to the behavior of the protein in solvent-free bilayers, where the closed state is dominant.
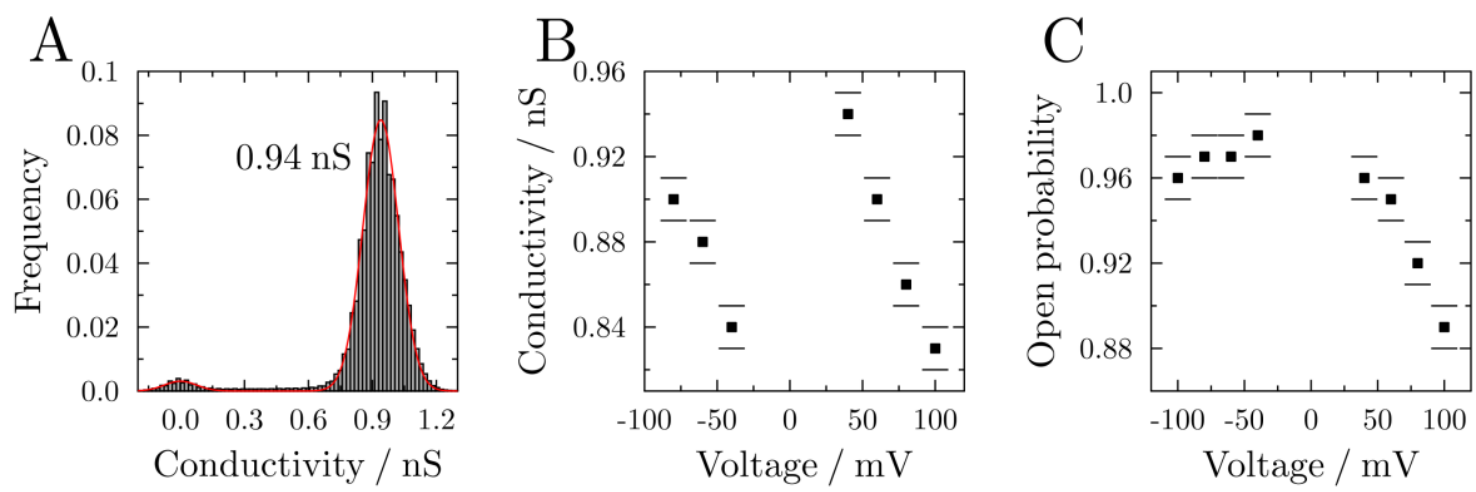

Figure 4.19. Voltage dependent conductivities and open probabilities of PorB wt in BLMs. (A) Exemplary point amplitude histogram of data recorded at $+40 \mathrm{mV}$.[156] (B) Voltage dependent conductivities. (C) Voltage dependent open probabilities. Due to an almost never occurring closed state, the represented conductivities values represent the difference between open monomer and open trimer and thus the conductivity of an open dimer (A and B). The open probabilities, however, belong to the fully open trimer, which is the preferred state at all voltages. Errors are the standard deviation. The measuring buffer contained $1 \mathrm{M} \mathrm{KCl,} 10 \mathrm{mM}$ HEPES, pH 7.5 and the bilayers were composed of DPhPC/Chol 9:1.

\subsubsection{Conductivity and open probability of PorB G103K}

The mutant G103K was also investigated in solvent containing membranes. After insertion, it showed the same behavior as the wild type: Most of the time the channel was completely open with only few gating events. The number of these events also increased with increasing applied voltage. For PorB G103K the exceptional case of very frequent gating after insertion was observed as well and from this data the conductivities and open probabilities were determined. An exemplary current trace recorded at $+40 \mathrm{mV}$ is displayed in Figure 4.20. In all 
cases of frequent gating, only opening and closing of a whole trimer (conductivity change of about $0.7 \mathrm{nS}$ ) could be observed, activity of a dimer or monomer was never detected. The associated point amplitude histogram demonstrates the dominance of the open sate.

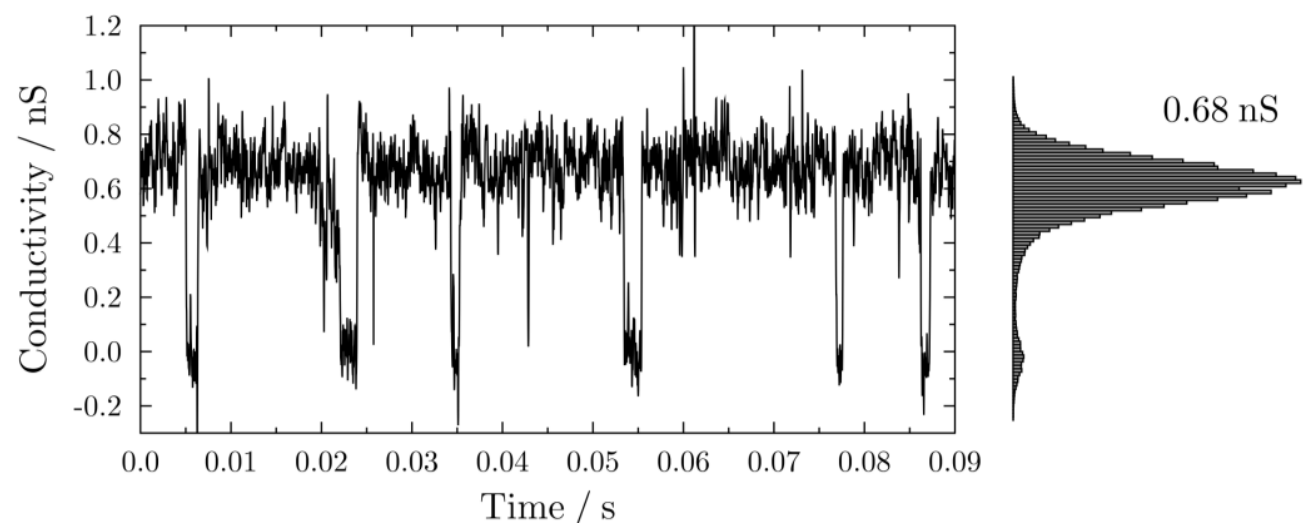

Figure 4.20. Exemplary conductivity trace with very frequent gating of PorB G103K recorded in BLMs at $+40 \mathrm{mV}$ and corresponding point amplitude histogram. Opening and closing of a whole trimer is visible. The lowest conductance level was set to zero. The measuring buffer contained $1 \mathrm{M} \mathrm{KCl}, 10 \mathrm{mM} \mathrm{HEPES}, \mathrm{pH} 7.5$ and the bilayer was composed of DPhPC/Chol 9:1.

For the conductivity event histogram, 4780 gating events at $+40 \mathrm{mV}$ were analyzed. The Gaussian fit results in one maximum at $G=(0.69 \pm 0.05) \mathrm{nS}$ (Figure 4.21) which is assigned to the trimer conductance and reflects the observation made for the conductivity traces. This value is only slightly lower than the one obtained in solvent-free bilayers.

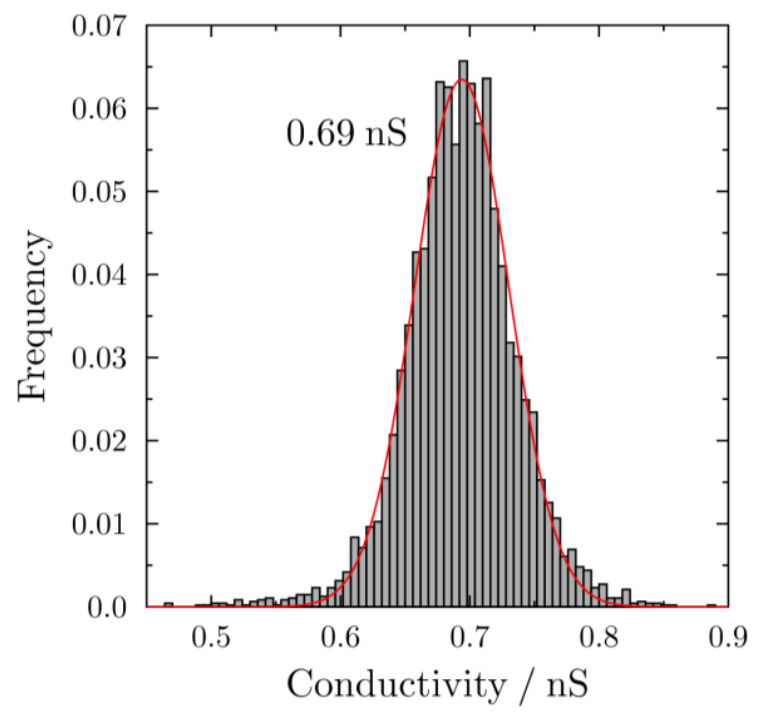

Figure 4.21. Conductivity event histogram of PorB G103K ( 4780 events) in BLMs at $+40 \mathrm{mV}$ and Gaussian fit (red line). The maximum is determined as $G=(0.69 \pm 0.05) \mathrm{nS}$. The measuring buffer contained $1 \mathrm{M} \mathrm{KCl}$, 10 mM HEPES, pH 7.5 and the bilayers were composed of DPhPC/Chol 9:1.

The open probability and conductivity of the PorB G103K trimer were determined for different applied voltages by analyzing the point amplitude histograms. The open probability could not be determined for negative voltages, because only few data points existed for the closed 
state and thus fitting of both maxima together was not possible. For the corresponding conductivities, both maxima were determined by separately fitting a Gaussian distribution to each data set and the trimer conductance was calculated as the difference of the centers. As for the wild type, all values were obtained by averaging the results of the individual conductivity traces and the errors are given as the standard deviation. Figure $4.22 \mathrm{~A}$ shows an exemplary point amplitude histogram for $+40 \mathrm{mV}$, revealing the open state as predominant.

In Figure $4.22 \mathrm{~B}$, the conductivities are plotted against the applied voltage. The same tendency as obtained for the wild type can be observed. At positive voltages, the values decrease with increasing voltage from $G_{\mathrm{T},+40 \mathrm{mV}}=(0.68 \pm 0.01) \mathrm{nS}$ at $+40 \mathrm{mV}$ to $G_{\mathrm{T},+100 \mathrm{mV}}=(0.63 \pm 0.01) \mathrm{nS}$ at $+100 \mathrm{mV}$. At -80 and $-100 \mathrm{mV}$ the channel closed very quickly and the values were very broadly distributed, so that a reliable determination of the conductivity was not possible. Thus, no tendency can be detected for negative voltages. The values obtained for -40 and $-60 \mathrm{mV}$, however, are lower than the ones determined at the corresponding positive voltages, as found for the wild type.

The open probabilities (Figure $4.22 \mathrm{C}$ ) show a decrease with increasing positive voltage, as already detected for the wild type with very similar values, ranging from $P_{\mathrm{o}, \mathrm{T},+40 \mathrm{mV}}=97 \%$ at $+40 \mathrm{mV}$ to $P_{\mathrm{o}, \mathrm{T},+100 \mathrm{mV}}=87 \%$ at $100 \mathrm{mV}$. At -40 and $-60 \mathrm{mV}$, the open probability can be assumed as about $100 \%$ and is therewith higher than at positive voltages, and higher than the ones of the wild type. The open state is preferred at all voltages for which a value could be determined.

$\mathrm{A}$

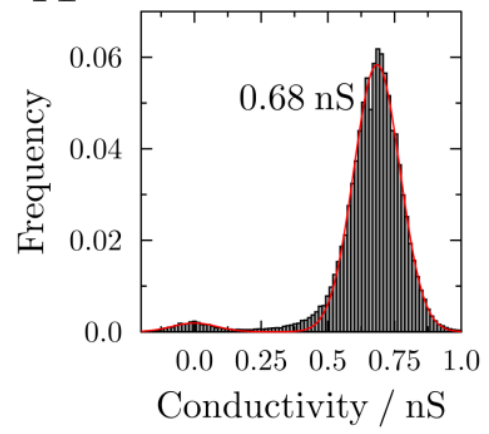

B

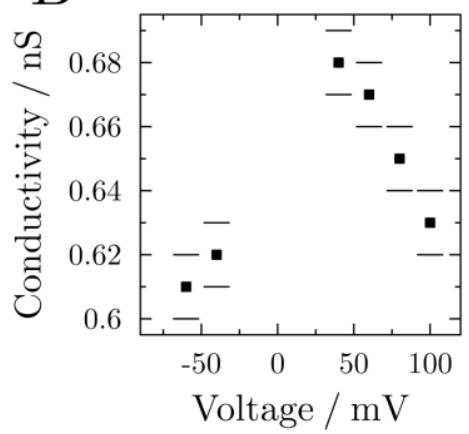

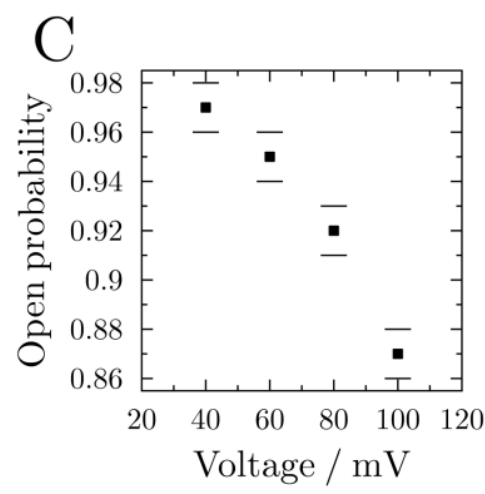

Figure 4.22. Voltage dependent conductivities and open probabilities of PorB G103K in BLMs. (A) Exemplary point amplitude histogram of the PorB G103K trimer in BLMs at $+40 \mathrm{mV}$. (B) Voltage dependent conductivities. (C) Voltage dependent open probabilities. Errors are the standard deviation. The open state is preferred at all voltages. The measuring buffer contained $1 \mathrm{M} \mathrm{KCl}, 10 \mathrm{mM}$ HEPES, pH 7.5 and the bilayers were composed of DPhPC/Chol 9:1.

\subsubsection{Conductivity and open probability of PorB G103D}

Data presented for the mutant G103D in black lipid membranes was mainly recorded by Svenja Kiehn during her Bachelor project. In BLMs, PorB G103D showed the same preference 
for the open sate as the other two porins. The channel was mainly open with only few gating events. For G103D as well some exceptional insertions were observed, followed by very frequent gating. In contrast to the wild type and G103K, in such cases often gating between monomer, dimer and trimer state appeared. An exemplary current trace recorded at $+40 \mathrm{mV}$ is presented in Figure 4.23 with the associated point amplitude histogram. The closed state at $0 \mathrm{nS}$ is not visible, as it only rarely occurred and there are thus too few data points. The most abundant state is the one of the open trimer at $1.00 \mathrm{nS}$, while dimer ( $0.64 \mathrm{nS})$ and monomer $(0.31 \mathrm{nS})$ are less preferred. An additional state was found at $0.14 \mathrm{nS}$.

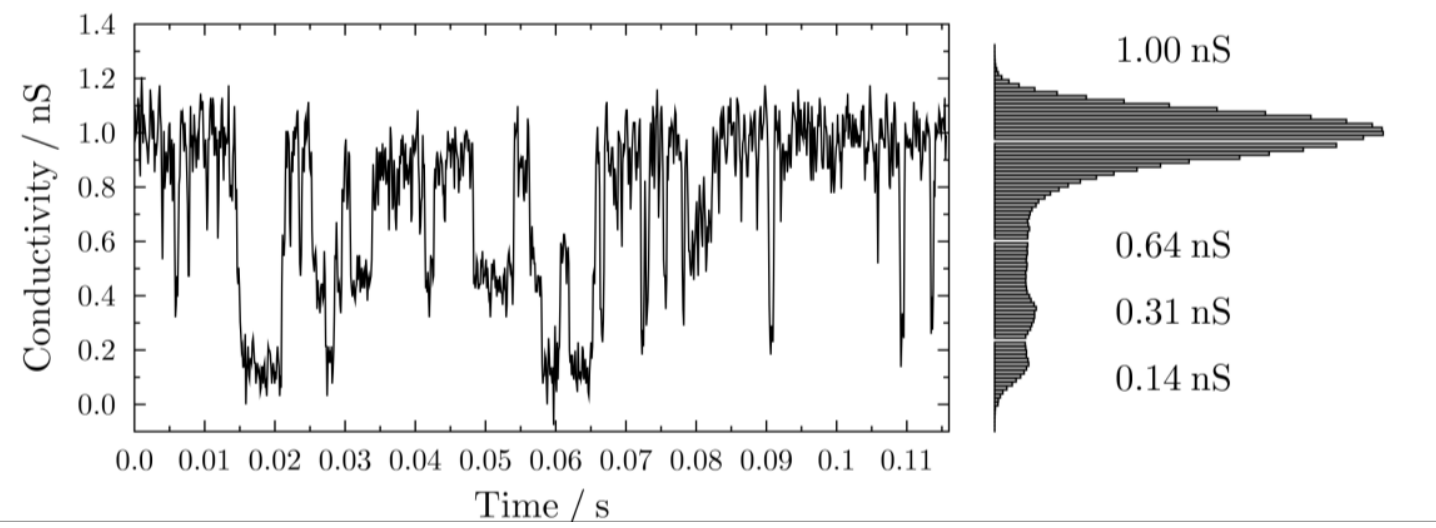

Figure 4.23. Conductivity trace with frequent gating of PorB G103D recorded in BLMs at $+40 \mathrm{mV}$. Trimer $(1.00 \mathrm{nS})$, dimer $(0.64 \mathrm{nS})$ and monomer state $(0.31 \mathrm{nS})$ are observed, together with an additional state at $0.14 \mathrm{nS}$. The lowest conductance level was set to zero. The measuring buffer contained $1 \mathrm{M} \mathrm{KCl}, 10 \mathrm{mM} \mathrm{HEPES}, \mathrm{pH} 7.5$ and the bilayers were composed of DPhPC.

The maximum at $0.14 \mathrm{nS}$ could be partially caused by the detergent LDAO, as events in this order of magnitude were observed as well in blank samples using solvent-free bilayers (compare Figure 4.3). However, in blank measurements using BLMs, no such events were found. A possible explanation could be that in absence of the protein, LDAO present in the buffer is not integrated into the bilayer. Only in the presence of the protein, LDAO is co-inserted into the bilayer. As the hydrophobic lipid chains presumably at least partially replace the detergent molecules in contact with the hydrophobic parts of the trimer, free LDAO is present in the bilayer and can have a destabilizing effect (compare Chapter 4.2.1.1).

From different measurements at $+40 \mathrm{mV}, 6149$ gating events were analyzed for the conductivity event histogram (Figure 4.24). The Gaussian fit reveals three maxima at $G_{\mathrm{M}}=(0.36 \pm 0.22) \mathrm{nS}, G_{\mathrm{D}}=(0.68 \pm 0.12) \mathrm{nS}$ and $G_{\mathrm{T}}=(0.94 \pm 0.12) \mathrm{nS}$. The values for the trimer and dimer are slightly lower than the ones obtained in solvent-free membranes, whereas the conductivity of the monomer is a bit higher. It has to be considered that the conductivity of the monomer in solvent-free membranes could only be determined from a point amplitude 
histogram of one measurement $\left(G_{\mathrm{M}}=(0.30 \pm 0.09) \mathrm{nS}\right)$, thus representing only a selection instead of a mean value of all events (see Chapter 4.2.1.4).

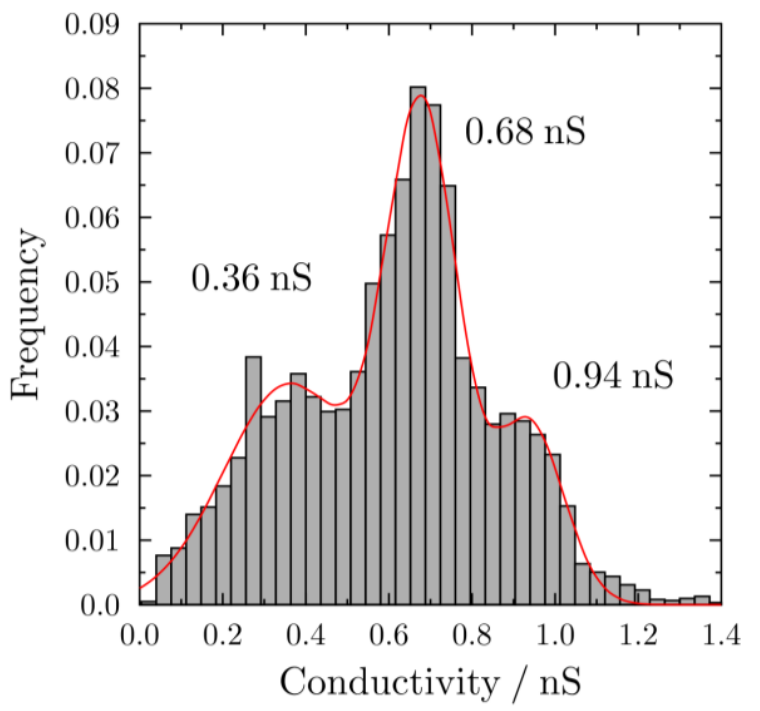

Figure 4.24. Conductivity event histogram of PorB G103D recorded in BLMs at $+40 \mathrm{mV}$ and Gaussian fit (red line). Out of 6149 events three maxima are found with conductivities of $G_{\mathrm{M}}=(0.36 \pm 0.22) \mathrm{nS}, G_{\mathrm{D}}=(0.68 \pm 0.12) \mathrm{nS}$ and $G_{\mathrm{T}}=(0.94 \pm 0.12) \mathrm{nS}$. The measuring buffer contained $1 \mathrm{M} \mathrm{KCl}, 10 \mathrm{mM}$ HEPES, pH 7.5 and the bilayers were composed of DPhPC/Chol 9:1 or pure DPhPC.

For the analysis of the trimer open probability and conductivity at different voltages only traces also showing dimer and monomer activity were available (Figure 4.25 A). Consequently, the obtained probabilities are lower than the ones determined for the wild type and the mutant G103K, where no transition between monomer, dimer and trimer state occurred, and cannot be compared directly. Nevertheless, a comparison of the voltage dependence is still possible. Only measurements at positive voltages were investigated, because conductivity traces at negative voltages showed too much noise to allow a proper analysis. In contrast to the wild type and the mutant G103K, at each voltage only one conductivity trace was recorded and thus no standard deviation can be indicated. A slightly larger error range than obtained for the values of PorB wt and G103K is therefore assumed.

The conductivities of the G103D trimer (Figure 4.25 B) decrease with increasing voltage like for the wild type and $\mathrm{G} 103 \mathrm{~K}$, from $G_{\mathrm{T},+40 \mathrm{mV}}=1.00 \mathrm{nS}$ at $+40 \mathrm{mV}$ to $G_{\mathrm{T},+100 \mathrm{mV}}=0.93 \mathrm{nS}$ at $100 \mathrm{mV}$.

The open probabilities (Figure 4.25 C) are as well reduced with increasing voltage, except for the value at $60 \mathrm{mV}$, which is the highest with $P_{\mathrm{o}, \mathrm{T},+60 \mathrm{mV}}=78 \%$. Assuming this value to be an outlier, the mutant G103D shows the same behavior as the other two porins. The open trimer is the predominant state. 

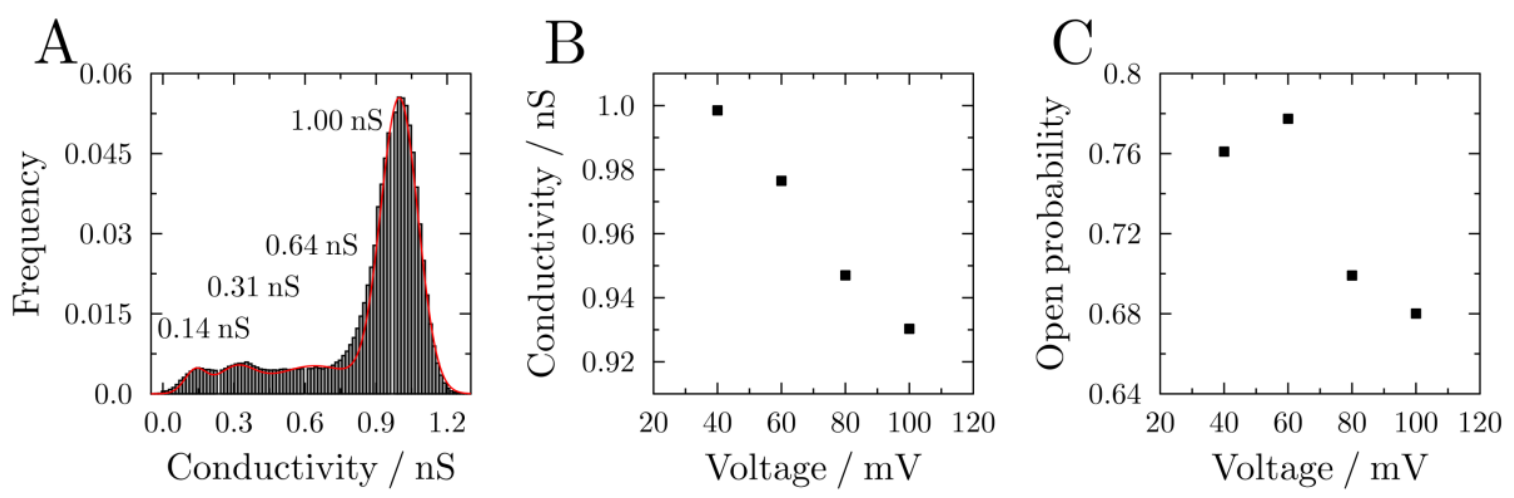

Figure 4.25. Voltage dependent conductivities and open probabilities of the PorB G103D trimer in BLMs. (A) Exemplary point amplitude histogram of data recorded at $+40 \mathrm{mV}$. (B) Voltage dependent conductivities. (C) Voltage dependent open probabilities. The analyzed traces showed not only trimer gating, but as well activity of dimer and monomer. The measuring buffer contained $1 \mathrm{M} \mathrm{KCl}, 10 \mathrm{mM}$ HEPES, pH 7.5 and the bilayers were composed of DPhPC.

\subsubsection{Noise analysis}

During the experiments, an exceptionally large open-channel-noise was observed for the mutant G103D (Figure 4.26 C). Therefore, a noise analysis was performed for all three porins.

Only BLMs are well suited for this analysis, because PorB shows large open probabilities in these membranes. The conductivity traces were chosen from the measurements recorded with the BLM-setup, as the following experiments under addition of ampicillin were carried out using this set-up.

Exemplary sections of conductivity traces of PorB wt, G103K and G103D recorded at $+40 \mathrm{mV}$ with the BLM set-up are presented in Figure 4.26. The traces of PorB wt and the mutant G103K show no difference, whereas the open-channel-noise of the mutant G103D is strongly increased.

A

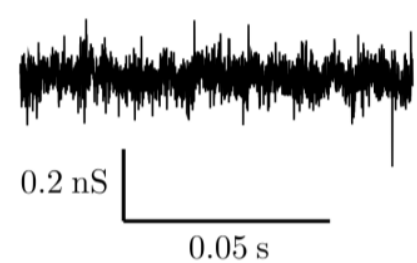

B

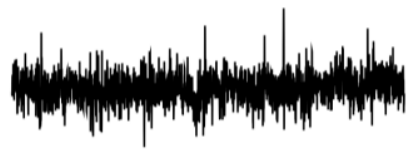

$\mathrm{C}$

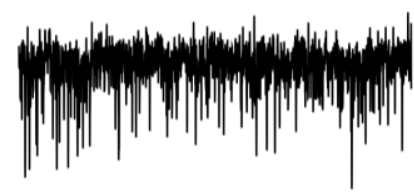

Figure 4.26. Open-channel-noise of PorB wt (A), G103K (B) and G103D (C). The conductivity traces were recorded at $+40 \mathrm{mV}$ using the BLM-setup. The conductance of the open state is $4 \mathrm{nS}$ for each trace. The measuring buffer contained $1 \mathrm{M} \mathrm{KCl,} 10 \mathrm{mM}$ HEPES, pH 7.5 and the bilayers were composed of DPhPC/Chol 9:1.

The noise analysis was performed using a MATLAB script (see Chapter 3.6.4.1) written by Ingo Mey (Steinem group, Institute of organic and biomolecular chemistry, University of Göt- 
tingen). After the determination of the baseline, systematically increasing thresholds (in percentage deviation from the baseline) are applied. All data points lying beyond the threshold below and above the baseline are chosen. The peak maxima within these data points are detected and a peak frequency is calculated. Finally, this frequency below (down) and above (up) the baseline is plotted against the applied threshold.

In Figure 4.27, the resulting frequency plots are depicted for the three proteins. As the threshold depends on the baseline, traces with the same open channel conductivity were compared. Consequently, in case of the mutant more trimers are inserted, because the conductivity is lower compared to the wild type and the mutant G103D (see Chapter 4.2.2.5). The thresholds range from $0 \%$ to $25 \%$ deviation from the baseline. At 0.0 , the maximum frequency - normalized to one - is reached. With increasing threshold, the frequency decreases to a minimum at 0.25 , this value was normalized to zero. The threshold, at which the frequency starts to increase, is a measure of the amplitude of the open-channel-noise. The open-channel-noise is the same for the wild type (blue) and the mutant G103K (red), visible by the almost identical curves. For both proteins, the frequencies start to rise rapidly at a threshold below 0.05 . The frequencies of G103D above the baseline behave the same way, but the frequencies below the baseline start to increase already at a threshold of about 0.15. This means that for G103D, the signals below the baseline have a much higher amplitude, reflecting the observations already made in the conductivity trace (Figure $4.26 \mathrm{C}$ ).

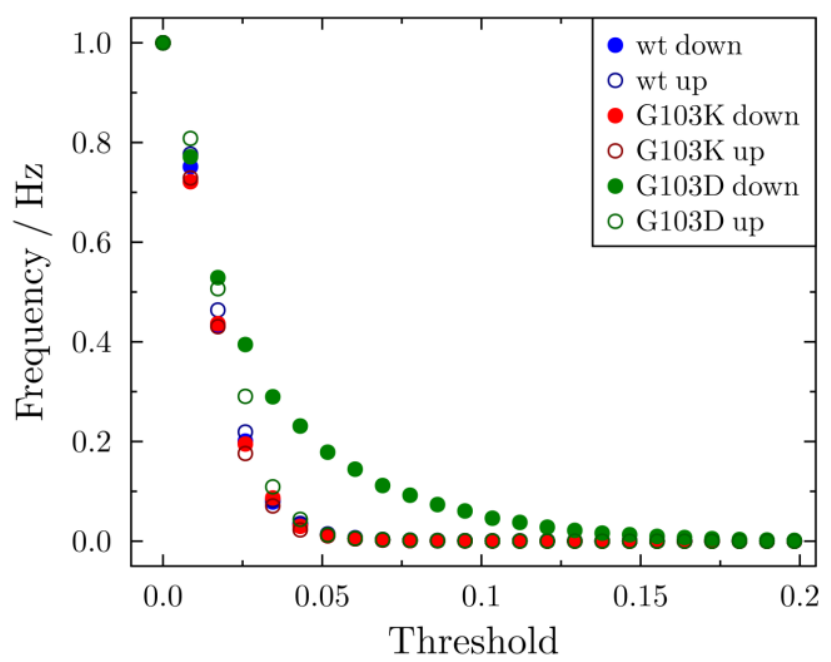

Figure 4.27. Noise frequencies of PorB wt (blue), G103K (red) and G103D (green). Frequencies are shown dependent on the applied threshold (percentage deviation of the baseline). The values for G103D below the baseline start to increase at a much lower threshold than for the other proteins, implying a larger noise amplitude. The measuring buffer contained $1 \mathrm{M} \mathrm{KCl}, 10 \mathrm{mM}$ HEPES, pH 7.5 and the bilayers were composed of DPhPC/Chol 9:1. 


\subsubsection{Comparison of PorB wild type and the mutants in BLMs}

\section{Channel conductance}

As already found in solvent-free membranes, the reduction of the channel conductance due to the mutation is more pronounced for PorB G103K than for PorB G103D. The determined voltage dependence of the conductance values is attributed to voltage dependent channel deformations.

A determination of trimer, dimer, and monomer conductivity from the event histogram was only possible for PorB G103D, because for wild type and G103K frequent gating was not observed between all open states. For the wild type, monomer and trimer conductivities could only be estimated from the point histogram of a small section of a conductivity trace recorded at $+40 \mathrm{mV}$ (Figure 4.17). Thus, they are no reliable values and only serve to correctly assign the found conductance of $G_{\mathrm{D}}=(0.95 \pm 0.07 \mathrm{nS})$ to the dimer. All values summarized in Table 4.3 belong to an applied voltage of $+40 \mathrm{mV}$.

Table 4.3. Conductivities of PorB wt, G103K and G103D in BLMs recorded at $+40 \mathrm{mV}$. Values were obtained from the conductivity event histograms, except for the PorB wt monomer and trimer $\left.{ }^{*}\right)$, which were taken from the point amplitude histogram of a small section of a conductivity trace (see Figure 4.17).

\begin{tabular}{c|ccc}
\hline \multicolumn{1}{c}{$\boldsymbol{G} / \mathbf{n S}$} & PorB wt & PorB G103K & PorB G103D \\
\hline Monomer & $0.34^{*}$ & - & $0.36 \pm 0.22$ \\
Dimer & $0.95 \pm 0.07$ & - & $0.68 \pm 0.12$ \\
Trimer & $\left.1.28^{*}\right)$ & $0.69 \pm 0.05$ & $0.94 \pm 0.12$ \\
\hline
\end{tabular}

Comparing the dimer conductivities, the value for the mutant G103D is lower than the one of the wild type. The unreliable value estimated for the wild type trimer supports this finding, whereas the estimated value for the wild type monomer shows no difference to the mutant monomer. The trimer conductivity of PorB G103K, is strongly decreased compared to the other two porins. This is in good agreement with the results of the measurements in solventfree membranes where especially the mutant G103K showed a decreased conductivity. The reduced conductivity of mutant porins is already discussed in Chapter 4.2.1.5.

The high stability of the BLMs allowed the determination of the conductivities at increasing applied voltages. A slight decrease with increasing positive voltage was found for all proteins, whereas a tendency at negative voltages could only be obtained for the wild type and revealed an increase with the applied voltage. 
A decrease of the channel conductance with increasing applied voltage is described for PorB Ngo and Nme, but this effect is caused by the voltage dependent closure of pores at higher voltages.[146,157,158] Thus, these results present the overall conductance of all inserted porins that are still open, not the voltage dependent conductivity of one gating porin as investigated in this study. Most studies show the relation of ion current to applied voltage, which is linear at low voltages and does not indicate a voltage dependence of the open channel conductance. ${ }^{[147,157,159]}$ For OmpF, however, the conductance of one fully open trimer was determined at two different $\mathrm{pH}$ values at voltages from $-200 \mathrm{mV}$ to $+200 \mathrm{mV} .{ }^{[103]} \mathrm{At} \mathrm{pH} 8.0$, a decrease of the conductivity was found between $-200 \mathrm{mV}$ and $+200 \mathrm{mV}$, more pronounced at lower salt concentrations.

Both porin features described - voltage-dependent gating/closure and voltage-dependent open-channel conductance - can be ascribed to field-induced conformational changes of the pores. Several channels are activated due to voltage-induced conformational changes, e.g., sodium, potassium and calcium channels.[160] For OmpF, different kinds of conformational changes are proposed to be responsible for gating, involving the internal loop 3, different surface loops or the $\beta$-barrel itself.[26] These conformational changes are discrete and follow the "all or nothing" principle, meaning that there are only two discrete states. Additionally, pores can undergo elastic structural deformations caused by the applied electric field, as shown for $\alpha$-hemolysin. ${ }^{[161]}$ A gradual change of the pore size with the applied voltage was found, detected by the variation of the pore conductance and the probability of blockage by 18-crown-16, which are both reduced with decreasing pore diameter. The voltage-dependent orientation discovered for certain residues at the outer surface of the $\beta$-barrel could influence the selectivity of $\alpha$-hemolysin and therewith as well its conductance.[162] Furthermore, this effect increases the radius of the pore size by $0.8 \AA$ when switching the applied potential from $+120 \mathrm{mV}$ to $-120 \mathrm{mV},[161]$ thus the sign of the voltage has a significant effect on the pore size. These effects could as well be responsible for the voltage dependency found in this study for the conductivity of PorB. A slightly decreasing pore size would explain the reduction of the conductance with increasing positive voltage obtained for all three porins, as well as the opposite tendency at applied negative voltages detected for PorB wt. If conformational changes of the $\beta$-barrel and reorientations of residues located at the surface of the barrel play the key role in this process, it is not surprising to find a similar voltage-dependence for the conductivity of all three proteins, as the mutation G103K and G103D are not located at the barrel itself, but at the internal loop. 


\section{Gating behavior}

The gating of the PorB wt and PorB G103K monomers is highly cooperative, whereas the reduced cooperativity in PorB G103D indicates an altered interaction between the monomers.

In most of the measurements all three PorB variants showed only occasional gating events, but in some cases, very frequent gating activity was observed. PorB G103K never showed the transition between the conductance state of monomer, dimer and trimer. For the wild type, only in few traces monomer gating was visible (see Figure 4.17). The mutant G103D, by contrast, exhibited in some experiments in addition to very frequent gating as well the transition between closed, monomer, dimer and trimer state. This behavior was already detected in a measurement on solvent-free membranes.

The influence of mutations at loop3 on the gating frequency described for OmpU and OmpC ${ }^{[153-155]}$ is not visible for PorB G103K and G103D, because very frequent gating was observed for both mutants and the wild type. As a high ionic strength in the measuring solution is discussed to enhance the frequency of transitions between open and closed state ${ }^{[155]}$ and $1 \mathrm{M} \mathrm{KCl}$ was present during the measurements, this effect might have masked possible difference due to the mutations. The use of a lower salt concentration might lead to a lower gating frequency and thus help to reveal a possible influence of the mutations.

The gating behavior of PorB wild type and the mutant G103K implies a strong cooperativity between the three monomers and confirms the results of the measurements using solventfree bilayers. Possible reasons are discussed in Chapter 4.2.1.5. On the contrary, the gating cooperativity of the mutant G103D monomers is strongly reduced (transition between monomer, dimer and trimer state occurred in several measurements), which confirms the tendency already found in one experiment on solvent-free membranes. The lower stability of the trimeric structure of the PorB mutants compared to the wild type detected in the no-SDS PAGE (see Chapter 4.1) as well indicates a changed interaction between the monomers due to the mutation. The increased frequency of gating caused by some mutations in $\mathrm{OmpC}$, mostly located near the barrel wall, was accompanied by a reduction of cooperativity.[154,155] The altered flexibility and positioning of loop3, affected by a putative network of hydrogen bonds and a salt bridge, is considered as possible cause for the altered frequency of gating [154] and might as well influence the cooperativity. As the mutations at position 103 of PorB (Nme) are far away from the barrel wall, these effects do probably not play a role for the PorB mutants. Nevertheless, one investigated OmpC mutation located at a position similar to 103 in PorB (Nme) presented an increased gating frequency and a loss of the cooperative behavior found in the wild type. ${ }^{[155]}$ In this case, the role of changes of the internal electrical field due 
to altered ionic interactions between charged residues within the constriction region is discussed. However, this theory would explain alterations induced by the mutation G103K rather than alterations due to the mutation G103D (see Chapter 4.2.1.5), because only the mutation G103K turned out to considerably change the pores surface potential. Furthermore, changes of the electrical field might very well influence the transition between open and closed state of a monomer, but can hardly be the only cause of altered interactions between the three monomers. For this, significant conformational changes due to the point mutation would be necessary. These changes would have to involve L2, which is strongly involved in the interaction between the monomers (compare Figure 4.16). Conformational changes of L2 due to mutations are reported neither for PorB from Nme nor for PorB from Ngo. For PorB from Ngo mutations at position 120 and/or 121 are just suggested to induce conformational changes of loop3.[48] Such conformational changes because of the mutations at position 103 in PorB Nme could neither be verified nor disproved up to now.[149]

\section{Open probabilities}

In BLMs, the open state of PorB is preferred and the open probability is not influenced by the mutations. The voltage dependence of the open probabilities could be caused by voltage dependent conformational changes that alter the energy barrier for the transition between open and closed state.

For the open probabilities of PorB wt and G103K, conductivity traces only showing gating between two states were evaluated. In case of PorB G103D, such traces could not be found, thus the open probabilities of the trimer were determined from traces presenting monomer and dimer activity as well. As a result, the open probabilities obtained for the G103D trimer are in general lower than the ones of the other two porins.

Open probabilities were investigated dependent on the applied voltage. At positive voltages, the probability decreases for all three proteins with increasing voltage. At negative voltages, the same tendency can be observed for the wild type, but the probabilities are in general higher and the dependency is less pronounced than at positive voltages. For G103K, the values at all negative voltages are about $100 \%$ and for G103D no data is available. In Table 4.4, the highest open probability at $+40 \mathrm{mV}$ and the lowest at $+100 \mathrm{mV}$ are summarized for the three porins. 
Table 4.4. Trimer open probabilities of PorB wt, G103K and G103D recorded at +40 and $+100 \mathrm{mV}$ in BLMs. For PorB wt and G103K, average values were determined from several conductivity traces and the errors are the standard deviations. In case of G103D, only one trace was available per voltage, therefore no error could be calculated.

\begin{tabular}{c|ccc}
\hline \multicolumn{1}{c}{$\mathbf{P}_{\mathbf{o}} / \mathbf{\%}$} & PorB wt & PorB G103K & PorB G103D \\
\hline $\mathbf{+ 4 0 ~} \mathbf{~ m V}$ & $96.3 \pm 0.4$ & $96.7 \pm 0.2$ & 76 \\
$\mathbf{+ 1 0 0 ~} \mathbf{~ m V}$ & $88.9 \pm 0.2$ & $86.9 \pm 0.8$ & 68 \\
\hline
\end{tabular}

For all three proteins, the open state is clearly preferred and the open probabilities decrease about 7-10\% between $+40 \mathrm{mV}$ and $+100 \mathrm{mV}$. For G103D, the trimer state is less preferred than for wild type and G103K, as monomer and dimer states occur as well.

Many porins show in BLMs as well a preference for the open state, at least at low voltages.[55,146,153,158,163,164] At a critical threshold potential, $V_{c}$, the channels start to close, either reversibly or permanently. This potential was determined as $\pm 150 \mathrm{mV}$ for $0 \mathrm{mpF}$ and $\pm 220 \mathrm{mV}$ for several OmpC mutants.[163,164] PorB from Neisseria meningitidis (Nme) and Neisseria gonorrhea $(\mathrm{Ngo})$ is reported starting to close at voltages above $\pm 60 \mathrm{mV}$.[47,158] In this study, transient closures of PorB wt Nme and the mutants, whose frequency increased with the voltage, were observed at voltages of $+40 \mathrm{mV}$ and above. Permanent closures were found at +100 to $+120 \mathrm{mV}$, sometimes already at $+80 \mathrm{mV}$. This is in good agreement with the curves of the voltage dependent open probability determined for PorB wt $\mathrm{Nme}$ and $\mathrm{Ngo}$, which approach zero at voltages above $\pm 100 \mathrm{mV}$.[47,50] However, these curves were determined from the described, often permanent, closures and therewith the open probabilities present a wider range from one to zero, contrary to the values calculated in this study, which are based on current traces with very frequent gating without permanent closures. Thus, the values presented here vary in a comparably small range.

Voltage dependent conformational changes, as described for $\alpha$-hemolysin[161,162], might not only alter the size and therewith the conductance of the pore (see discussion above), but could influence the open probability as well. The mechanism of voltage gating in porins is not solved yet, but many existing theories involve conformational changes. They include a motion of loop3 into the constriction region,[22] rearrangements of the charges at the constriction zone, ${ }^{[23,24]}$ movement of surface loops into the pore ${ }^{[25]}$ or gating as an intrinsic property of the barrel itself, e.g., by large structural deformations. ${ }^{[26,27]}$ An alteration of the pore conformation can be imagined to reduce or increase the energy necessary to switch between open and closed conformation and thus influence the frequency of events. Further, a large imbalance of the barriers for the transition from the closed to the open state and the opposite direction 
might be the result of a different conformation, so that even a change of the energetically preferred state between open and closed could be the consequence. In molecular dynamics simulations of $\mathrm{OmpF}$, for example, a higher flexibility of loop3 in the presence of an electric field than without one was detected..[26] A higher flexibility of loop3 could reduce the energy barrier for conformational changes that might be involved in the transition between open and closed state. Furthermore, the strength of this field and therewith the applied voltage might influence the degree of the loop3 flexibility. A similar scenario can be envisioned for the external loops. In loop6 and loop8 of OmpF a lysine is located near and at their hairpins, which are discussed to move due to an applied electric field, based on the findings in a porin from $H$. influenza. ${ }^{[25,26]}$ For $\alpha$-hemolysin, a strong dependence of the pore size and the conformation of one particular residue on the sign of the voltage were detected.[162] A strong influence of the sign of the applied voltage on the pore conformation would explain the distinctly increased open probabilities, near $100 \%$, found for PorB wt Nme and G103K at negative voltages. Such tendencies are not described for PorB up to now, open probabilities were found to have a symmetric curve for negative and positive voltages. ${ }^{[47,50]}$

In contrast to the observations made in solvent-free bilayers (see Chapter 4.2.1.5), PorB wt Nme and the mutant G103K present nearly identical open probabilities in BLMs (see Table 4.4). The open probabilities obtained for the mutant G103D are lower due to the presence of monomer and dimer state in addition to the trimer state. Hence, only the tendency of voltage dependence can be compared. The decrease of the probabilities between +40 and $+100 \mathrm{mV}$ is about 7-10 \% for all three porins. As a conclusion of these results, the open probability of PorB Nme in BLMs is not altered by the point mutations G103K and G103D, contrary to findings described for PorB Ngo and other porins and their mutants, ${ }^{[49,153-155]}$ as discussed in Chapter 4.2.1.5.

\section{Open channel noise}

The increased open channel noise of PorB G103D compared to PorB wt and PorB G103K is ascribed to pKs shifts of ionizable residues within the pore and electrostatic repulsions between residues at the internal loop.

The mutant PorB (Nme) G103D exhibits an increased open channel noise compared to the wild type and the mutant G103K (see Figure 4.26). An enhanced peak frequency was already found at a threshold of 0.15 , i.e., $15 \%$ deviation from the baseline (see Figure 4.27), which corresponds to a noise amplitude of 0.4-0.5 nS (baseline level about $4 \mathrm{nS}$ ). For the other two 
porins, events were detected at less than $5 \%$ deviation from the baseline, thus the noise amplitude is below $0.2 \mathrm{nS}$.

Several mutants of PorB $\mathrm{Ngo}$, among them mutations corresponding to those investigated in this study, as well exhibited an increased open channel noise. ${ }^{[4]}$ As possible causes, fluctuations within the pores and rapid transitions between different (sub-)conducting states are suggested. For OmpF a dependence of the open channel noise on the $\mathrm{pH}$ value of the bath solution is discussed to originate from $\mathrm{pKs}$ shifts of ionizable residues within the pore. ${ }^{[103]} \mathrm{An}$ alteration or introduction of ionizable groups, as it is the case for the mutation G103D, might also lead to such shifts of the neighboring residues and thus induce an increased open channel noise. The substitution of a negatively charged by a positively charged amino acid (D127K) at the internal loop of $\mathrm{OmpF}$ resulted in enhanced current fluctuations of the open channel.[165] Besides changes of the ionization state of this residue causing the fluctuations, electric repulsion between the two positive residues Lys127 (loop3) and Arg167 (loop4) are proposed to destabilize the position of loop3 and raise its flexibility. Upon the mutation G103D, another negative residue is added to the cluster of negatively charged amino acids at loop3 (see Figure 4.28). This might lead to electrostatic repulsions, for example between Asp103 and Asp104 or Glu116. A consequence might be a higher flexibility of loop3 or even a movement between the two parts of the loop and could be the reason for the increased open channel noise. The substitution G103K, however, would lead to electrostatic attraction between residue 103 and 104 or 116, thus no increased fluctuations of the open channel conductance are observed. 

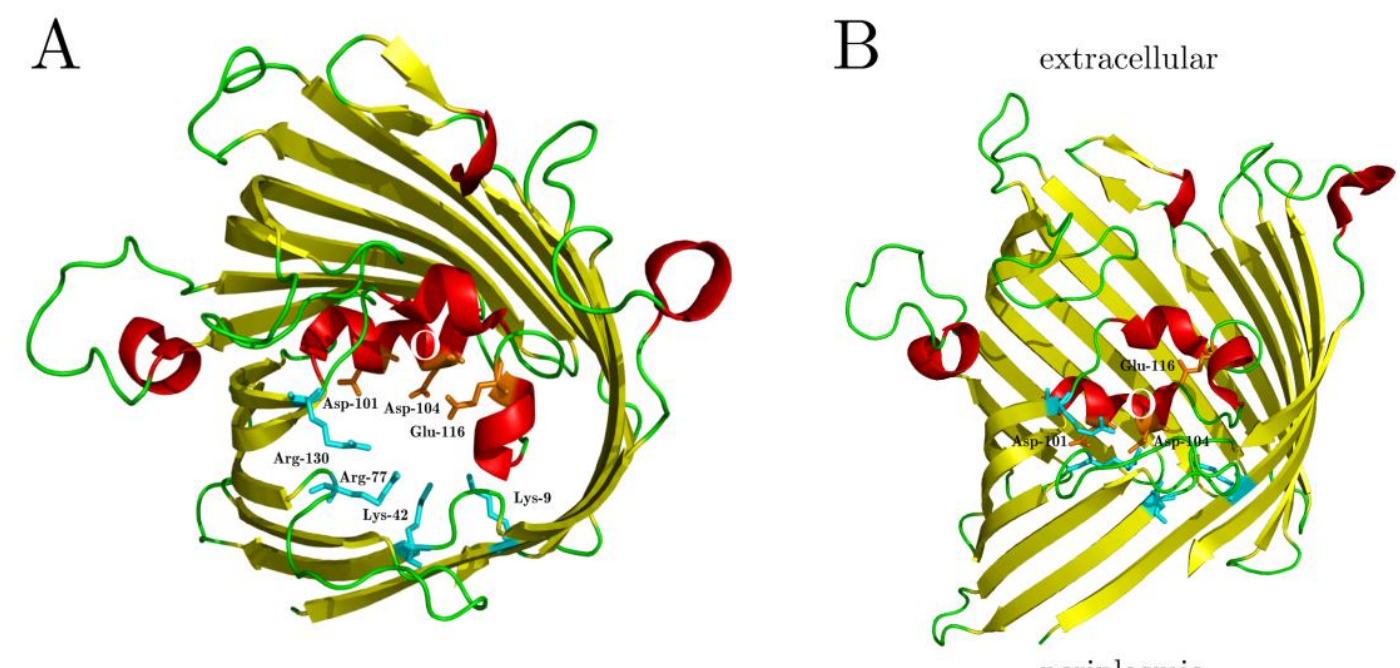

Figure 4.28. Crystal structure of a PorB (Nme) wt monomer. View from the extracellular side (A) and the membrane side (B). The beta barrel is shown in yellow, loops in red and turns in green. Negatively charges residues at loop3 are depicted in orange and positively charged residues at the opposed barrel wall in cyan. The approximate position of residue 103 is marked with a white circle. (PDB ID: 3VZT) ${ }^{[76]}$

Further, the raised open channel noise of PorB G103D could be subgating, i.e., gating between states occurring in addition to monomer, dimer or trimer state (subconductance states), or flickering, i.e., very fast closing events with amplitudes corresponding to one of the main conductance states. As the amplitude determined for the noise of the mutant is 0.4-0.5 nS, it is within the range of the monomer conductivity obtained from the conductivity event histogram in BLMs (see Figure 4.24). A subconductance state, as found for OmpF wild type, would be expected to be of lower conductivity than one monomer.[166] Hence, subgating can be excluded. Flickering is more likely to be the reason for the open channel noise of PorB G103D, not only because of the amplitude matching to the conductivity of one monomer. Further, the reduced gating cooperativity between the monomers detected for the mutant (compare discussion above) can lead to increased activity of one single monomer. Flickering of the wild type pores ${ }^{[166,167]}$ is reported for other porins, but the suggested causes are the same as described above for the increased gating frequencies of mutants.[153-155] However, the time scale of these flickering events is similar to those of the very frequent gating observed for all three PorB variants in this study, whereas the flickering of PorB G103D happens much faster (Figure 4.29). Therefore, the mentioned pKs-shifts and electrostatic repulsions appear to be more probable as source of the increased open channel noise of PorB G103D. 


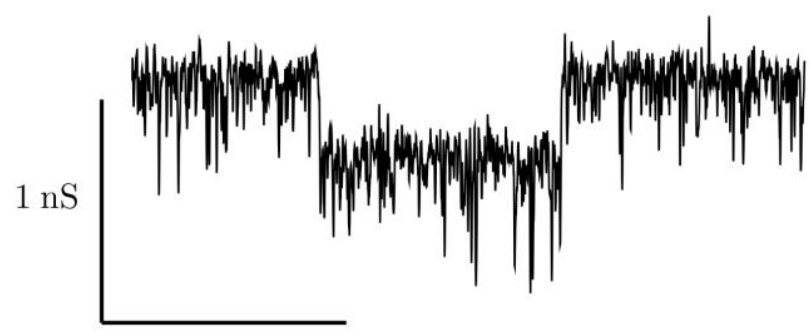

$0.02 \mathrm{~s}$

Figure 4.29. Section of a conductivity trace of PorB G103D inserted into a BLM, recorded at $+40 \mathrm{mV}$. The gating of the monomer presents a duration of about $0.02 \mathrm{~s}$, whereas the flickering happens at a much shorter time scale.

\subsubsection{Comparison of PorB properties in solvent-free bilayers and BLMs}

Because of the different advantages and disadvantages of solvent-free bilayers and black lipid membranes (BLMs) (see Chapter 3.6.2), both systems were used in parallel to ensure a profound characterization of PorB wild type (Nme) and the mutants G103K and G103D. Special attention is directed to the influence of the organic solvent present in BLMs on the protein properties, i.e., conductivity and open probability. Further, based on the results it should be decided which system is most-suited for the investigation of the porin-ampicillin interaction (see next chapter). In the following, the electrophysiological properties for each individual porin are compared for solvent-free and solvent-containing system, as well as the differences detected between the three variants.

\section{Open channel conductance}

The open channel conductivities $(G)$ of PorB wt, G103K and G103D obtained in solvent-free bilayers and BLMs are summarized in Table 4.5. In solvent-free membranes, values for all three states, i.e., monomer, dimer and trimer, were found, whereas in BLMs only for G103D all values could be determined reliably. It has to be noted, that the conductivity for the G103D monomer in solvent-free membranes was not obtained from the conductivity event histogram, but from the point amplitude histogram of one single conductivity trace. Therefore, the value bears a larger uncertainty than indicated by the errors (i.e., full width at half maximum, FWHM). The monomer and trimer values for the wild type in BLMs were only estimated from a small section of a current trace and are thus indicated without error and only listed for the sake of completeness. 
Table 4.5. Monomer, dimer and trimer conductivities of PorB wt, G103K and G103D recorded at $+40 \mathrm{mV}$ in solvent-free bilayers and BLMs. Marked values ${ }^{*}$ ) could only be obtained from point amplitude histograms instead of conductivity event histograms.

\begin{tabular}{l|ccc}
\hline \multicolumn{1}{c}{$\boldsymbol{G} / \mathbf{n S}$} & & Solvent-free & BLM \\
\hline \multirow{3}{*}{ PorB wt } & Monomer & $0.39 \pm 0.18$ & $0.34^{(*)}$ \\
& Dimer & $0.84 \pm 0.26$ & $0.95 \pm 0.07$ \\
& Trimer & $1.48 \pm 0.46$ & 1.28 (*) $^{*}$ \\
\hline \multirow{2}{*}{ G103K } & Monomer & $0.11 \pm 0.05$ & - \\
& Dimer & $0.34 \pm 0.08$ & - \\
& Trimer & $0.73 \pm 0.14$ & $0.69 \pm 0.05$ \\
\hline \multirow{2}{*}{ G103D } & Monomer & $0.30 \pm 0.09\left(^{*}\right)$ & $0.36 \pm 0.22$ \\
& Dimer & $0.73 \pm 0.19$ & $0.68 \pm 0.12$ \\
& Trimer & $1.14 \pm 0.13$ & $0.94 \pm 0.12$ \\
\hline
\end{tabular}

Comparing the conductivities of each porin, the values only slightly vary between the two membrane systems. Considering the FWHMs, the conductivities determined for the three PorB variants are comparable for both membrane systems. Furthermore, in both systems the conductance values of the mutant G103K are notably lower than the ones of the other two porins.

\section{Gating Cooperativity}

Concerning the gating cooperativity of the monomers, no differences between solvent-free and solvent-containing membrane system were found. PorB wt and G103K show in both systems a highly cooperative behavior, while PorB G103D presents a strongly reduced cooperativity and more independent gating of the monomers. Consequently, the organic solvent present in the BLMs seems not to influence the interaction of the monomers or lead to a less tight association between them.

\section{Open probabilities}

Only the open probabilities $\left(P_{0}\right)$ of the trimer are compared, because dimer and monomer activity was never observed for PorB G103K in BLMs. The values obtained at $+40 \mathrm{mV}$ are listed in Table 4.6. As different measurements of one protein in solvent-free bilayers resulted in strongly varying open probabilities, no meaningful error can be indicated and absolute values are not compared. Nevertheless, the general tendency between solvent-free and solvent- 
containing system is still distinct. Besides the value of PorB G103D in BLMs, all probabilities were obtained from experiments showing only gating between to states.

Table 4.6. Open probabilities of PorB wt, G103K and G103D recorded at $+40 \mathrm{mV}$ in solvent-free membranes and BLMs. Due to the large variance between the results of the different measurements in solvent-free bilayers, no errors are indicated. The value for G103D in BLMs was in contrast to all others obtained from a conductivity trace showing monomer, dimer and trimer activity. ${ }^{*}$

\begin{tabular}{c|cc}
\hline $\mathbf{P}_{\mathbf{o}} / \boldsymbol{\%}$ & Solvent-free & BLM \\
\hline PorB wt & 21 & 96 \\
G103K & 39 & 97 \\
G103D & 18 & $76(*)$ \\
\hline
\end{tabular}

In the solvent-free and solvent-containing system, opposed tendencies were found for the open probabilities. While in solvent-free membranes all three porins predominantly adopt the closed state, in BLMs the open state is clearly preferred.

In summary it can be said, that, despite the slight variances of the conductivities, the only striking difference between solvent-free membranes and BLMs concerning the proteins' electrophysiological properties are the opposed open probabilities. This observation is attributed to the versatile effects of organic solvents on membrane properties that in turn influence protein properties and function.

Although there is a lot of research about the influence of organic solvent on the function of enzymes in solution, ${ }^{[106,168-170]}$ only few studies comparing proteins inserted into solvent-free and solvent-containing membranes exist. The characterization of subconductancte states found for $\mathrm{OmpF}$ was performed in protein containing liposomes (solvent-free) and BLMs in parallel.[166] No significant difference concerning the conductance levels or the frequency distribution of the substates was found. The authors note, that small conductance values might be difficult to detect in BLMs due to the high filter frequencies often used in this technique and conclude that a combination of both techniques is essential for a solid electrophysiological characterization. In another publication, $\mathrm{OmpF}$ was used as benchmark to compare two solvent-containing and one solvent-free patch-clamp method.[171] In all three systems, the porin stayed predominantly in the open state at the applied voltage of $-50 \mathrm{mV}$ and exhibited comparable conductance values. Neither in these two studies, nor in any other publication, a strong dependence of the pores' open probability on the presence of organic solvents in the membrane is described. 
However, various effects of organic solvents on the properties of the bilayer are known. Solvent-free membranes show a higher membrane tension than solvent-containing ones. This is possibly caused by an increased lateral diffusion in presence of organic solvents that might increase the membrane elasticity.[66] Furthermore, the thickness of a bilayer is influenced by the presence of an organic solvent and its chain-length.[172] Solvent-containing membranes present an increased thickness compared to the natural ones, i.e., solvent-free bilayers. This effect is reported to reach a maximum when the solvent is decane and to decrease with the length of the solvent's hydrocarbon chain, as it gets closer to the length of the lipid chains. The reason for this observation is the influence of the solvent's chain length on the conformation of the lipid chains. The increase in hydrocarbon chain length of the solvent leads to a reduction of the lipid chains' extension. Consequently, the bilayer becomes thinner and the fraction of solvent within the layer is decreased. At a solvent chain length close to the one of the lipids, the solvent is nearly not present within the bilayer anymore and the thickness is close to the one of a natural membrane.

The change of membrane properties due to the presence of an organic solvent might influence the interaction between bilayer and inserted protein and thus alter the protein properties and function. For example, mechanosensitive channels change their open probability due to membrane stress.[173] The open lifetime of gramicidin channels is described to depend on membrane thickness and tension. ${ }^{[174,175]}$ For this peptide channel, a correlation of the hydrophobic mismatch between membrane thickness and channel length to its electrophysiological properties is widely known. ${ }^{[176,177]}$ The activity of several other membrane proteins is reported to depend on the membrane thickness and/or the hydrophobic mismatch as well. The $\left(\mathrm{Ca}^{2+}, \mathrm{Mg}^{2+}\right)$ ATPase from sacroplasmic reticulum shows an optimum activity at carbon chain length of 20 atoms.[105] The addition of decane to a bilayer containing lipids of this chain length reduces ATPase activity, whereas the removal of decane from bilayers containing lipids of lower chain length leads to a completely inactive protein. A chain-length dependent activity was also found for the human erythrocyte hexose transporter,[178] the leucine transport system of Lactococcus lactis ${ }^{[179]}$ and Pseudomonas aeruginosa, ${ }^{[180]}$ the cytochrome $c$ oxidase and $\left(\mathrm{F}_{1}, \mathrm{~F}_{0}\right)$ ATPase complex.[181]

The importance of the hydrophobic coupling between the bilayer's hydrophobic thickness and the hydrophobic length of the inserted protein has been discussed in detail.[177] In case of a hydrophobic mismatch, bilayer and protein have to adapt to each other in order to avoid the high energetic cost of an enhanced contact between hydrophobic and hydrophilic residues /surrounding water. As a consequence, either the local membrane thickness or the protein length are altered. The latter can happen by small adjustments of amino acid side chains 
or even by a change of the protein conformation. For most transmembrane proteins with more than one known conformation, conformational changes involve the boundary between bilayer and the proteins' transmembrane domains.[177] The only $\beta$-barrel listed, OmpG, presents one of the exceptions, where the conformational change of an external loop is triggered by the change from a neutral to an acidic $\mathrm{pH}$-value.[182]. However, as the exact gating mechanism of porins and the conformational changes involved are not yet established, an influence of the hydrophobic coupling on the gating of these proteins cannot be excluded. For example, conformational changes of the external loops might be caused by an alteration of the bilayer's hydrophobic thickness. The organic solvent present in the BLMs prepared for this project, decane, is described to have a very strong impact on the thickness of a lecithin bilayer, increasing it from 31.4 to $48 \AA$, compared to the situation with virtually no solvent left.[172] The hydrophobic core of a pure DPhPC bilayer is about $45 \AA$ thick, the thickness including the headgroups was simulated as $60 \AA .{ }^{[67]}$ The total length of a PorB monomer can be estimated as $50 \AA$ (PDB-ID: 3VZT).[35,76] whereas the hydrophobic length is not known. Increasing the thickness of the DPhPC bilayer by adding decane alters the hydrophobic coupling between membrane and protein and might influence the preferred conformation of PorB. This would explain the opposed preference of open and closed state in the two investigated membrane systems.

The results show that, besides the open probability, there is no substantial difference between the protein properties determined in solvent-free bilayers and BLMs. The electrophysiological characterization of the interaction between $0 \mathrm{mpF}$ and various $\beta$-lactam antibiotics was performed before, using a solvent-free and two solvent-containing methods. ${ }^{[171]}$ Comparable results are reported for all three systems. Thus, it can be assumed that interactions between antibiotics and pores are not influenced by residual organic solvent in the membrane and from this point of view, both methods described can be used equally to investigate the interaction between PorB and ampicillin. 


\subsection{Investigation of the interaction with ampicillin}

After the electrophysiological characterization of PorB wild type and the two mutants, the proteins were investigated in the presence of ampicillin to identify possible influences of the point mutations on the interaction with the $\beta$-lactam antibiotic. In electrophysiological measurements, the binding of molecules to an open channel or pore can be detected by a short blockage of the conductance, caused by an interruption of the ion flux. The interaction between pore and antibiotic is characterized by the blockage amplitude (the extent to which the channel conductance is reduced), the residence time (the time the antibiotic is bound in the pore) and the blockage frequency (the rate of interactions).

These three characteristics are used in the following to compare the interaction of ampicillin with PorB wild type and the mutants. On the one hand, they are investigated dependent on the antibiotic concentration in order to compare the results with existing literature. On the other hand, the voltage dependence of the described characteristics is compared between the three proteins. As the pore's inner electric field plays a key role in antibiotic binding and permeation (see Chapter 1.2.2), the applied voltage might influence the interaction between pore and antibiotic by altering this electric field. This influence could be different for PorB wild type and the mutants, because the mutations introduce different charges and might thus as well alter the pore's inner electric field. In order to distinguish between ampicillin induced events and events caused by the membrane or the proteins, blank samples were recorded at different voltages after each protein insertion.

For this part of the project, black lipid membranes were used, as in these bilayers the open state of PorB is greatly favored (see Chapter 4.2.2.5). The experimental conditions were the same as for the measurements in BLMs without ampicillin. After protein insertion (addition to cis side), the required amount of ampicillin stock solution (25 mM in measuring buffer of proper $\mathrm{pH}$ ) was added to both chambers. In addition to $\mathrm{pH} 7.5$, at which $60 \%$ of the ampicillin molecule are negatively charged (see Chapter 3.3), the interaction was investigated at pH 6 as well, because at this $\mathrm{pH}$ the antibiotic exists almost exclusively as zwitterion. This way, it can be distinguished which effects can be ascribed to the negative charge of ampicillin, especially regarding the voltage dependence. 


\subsubsection{Conductivity traces in the presence of ampicillin and power spectral densities}

PorB wt and the mutants G103K and G103D were investigated in the presence of ampicillin.

In Figure 4.30, exemplary current traces recorded at $+80 \mathrm{mV}$ and at $\mathrm{pH}$ of 7.5 in the absence $(\mathrm{A}, \mathrm{C}, \mathrm{E})$ and presence $(\mathrm{B}, \mathrm{D}, \mathrm{F})$ of $1 \mathrm{mM}$ ampicillin are presented. In case of PorB wt and G103K, extremely short interruptions of the channel conductivity were observed after addition of the antibiotic (see Figure 4.30 B and D and red box) that never appeared in the absence of ampicillin. Due to the increased open-channel-noise of PorB G103D, no difference between the conductivity traces before and after ampicillin addition were detectable by eye (see Figure 4.30 E and F). However, for comparison, the traces of PorB G103D were equally analyzed as described in Chapter 4.3.2.1.

A

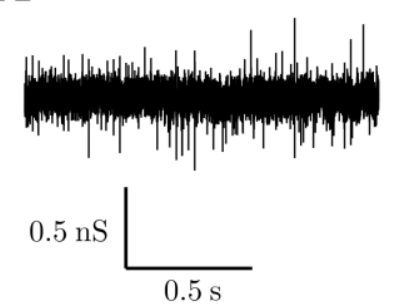

$\mathrm{B}$

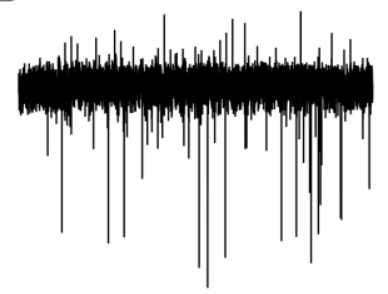

C
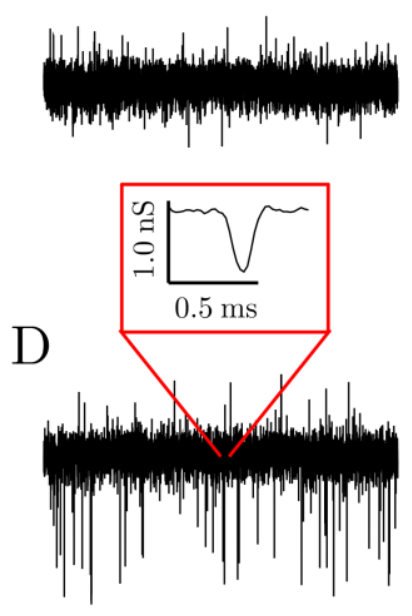

$\mathrm{E}$

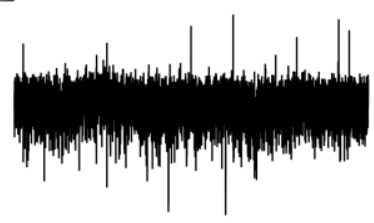

F

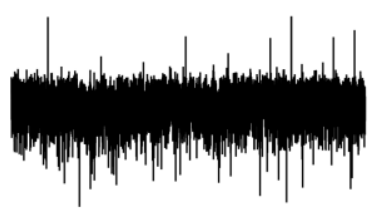

Figure 4.30. Exemplary conductivity traces of PorB wt (A and B), G103K (C and D) and G103D (E and F) in the absence (A, C, E) and presence of $1 \mathrm{mM}$ ampicillin (B, D, F). In case of PorB wt and G103K, short interruptions of the open-channel-conductivity are visible in the presence of ampicillin. A zoom-in of a single blockage event is presented in the red box. Traces were recorded at $+80 \mathrm{mV}$ and $\mathrm{pH} 7.5$. The measuring buffer contained $1 \mathrm{M} \mathrm{KCl}$, $10 \mathrm{mM}$ HEPES and the bilayers were composed of DPhPC/Chol 9:1.

Usually, the characteristics of channel blockage, i.e., antibiotic residence time and blockage frequency, are obtained by generating power spectra of the current signals. Power spectral densities are the amplitude of the current noise dependent on the frequency. By a Lorentzian fit, the blockage parameters can be determined.[52,55]

Figure 4.31 shows exemplary power spectral densities of PorB G103K in absence (blue) and presence of $1 \mathrm{mM}$ ampicillin (red). There is no difference between the two spectra, whereas for OmpF and ampicillin, as well as for Omp36 and ertapenem, densities with and without $\beta$ lactam antibiotic are reported to differ by several orders of magnitude.[52,55] 


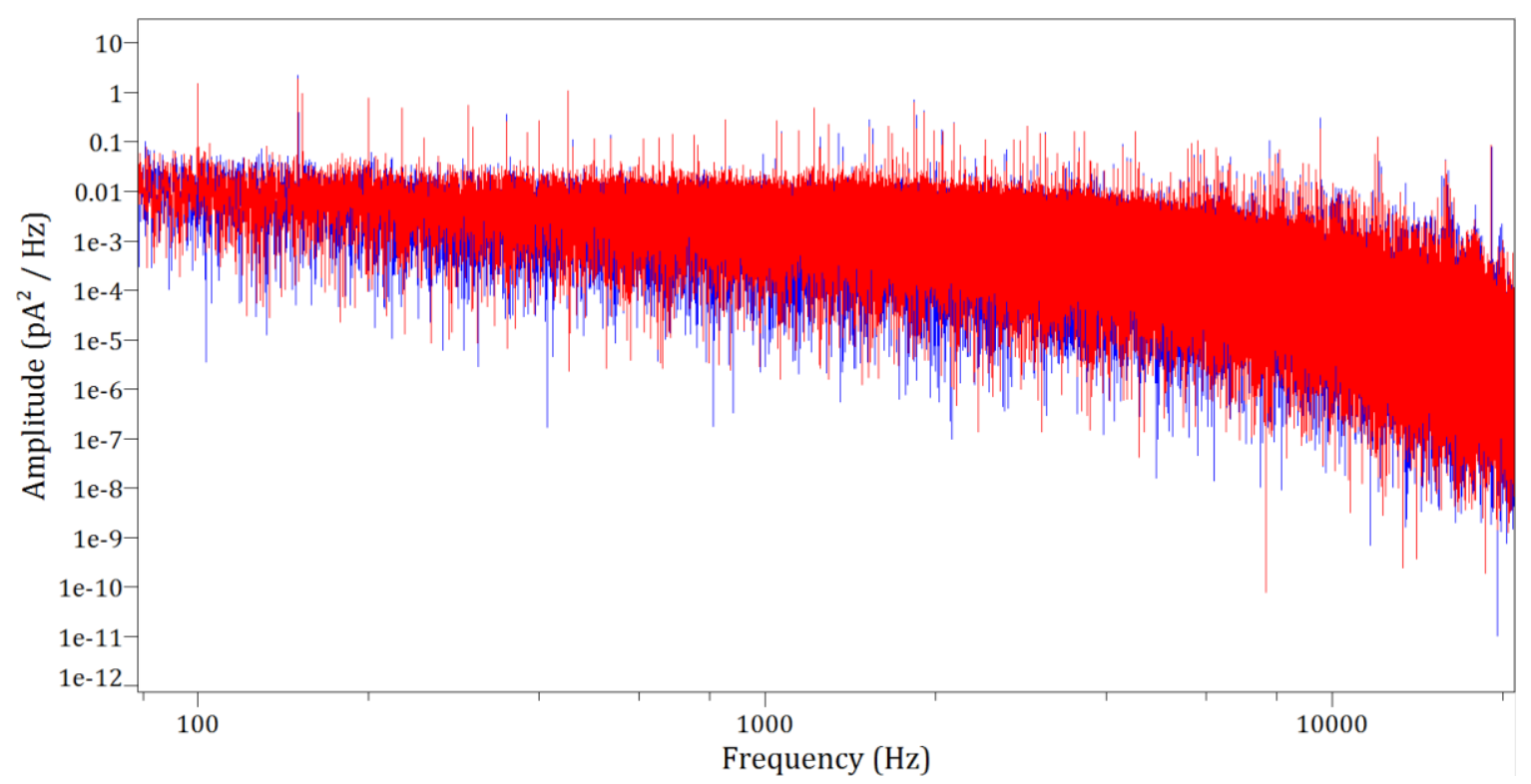

Figure 4.31. Exemplary power spectral densities of PorB G103K in the absence (blue) and presence of $1 \mathrm{mM}$ ampicillin.

In literature, antibiotic residence times are reported to be longer, leading to well resolved levels of the blocked channel, while for PorB and ampicillin the residence times are so short that only extremely sharp blockage signals ("spikes") are visible. In addition, the number of blockage events per second is visibly lower for PorB and ampicillin, likely due the lower antibiotic concentrations used in this project. The combined effect of the relatively high noise of the used BLM setup (see Chapter 3.6.3.2), the low number of events and the short residence times apparently lead to identical power spectra of experiments without and with ampicillin. For Omp36 and $10 \mathrm{mM}$ ertapenem, the difference of porin in the absence and presence of the antibiotic lies within 0.001 and $0.1 \mathrm{pA}^{2} / \mathrm{Hz},{ }^{[55]}$ which is the noise range of a PorB trace without ampicillin. Consequently, differences of this order of magnitude would not be detectable in the power spectral density. Considering the 10 -fold lower antibiotic concentration present in the PorB experiments, the variation between the two spectra can be expected even less pronounced than in the case of Omp36.

As the method of power spectral densities is obviously not suited for the analysis of the interaction between ampicillin and PorB, the new routine JULES (JUmp Local dEcovolution Segmentation filter (see Chapter 3.6.4.2),[110] developed by Florian Pein (Munk group, Institute for Mathematical Stochastics, University of Göttingen), was applied. 


\subsubsection{Analysis using the routine JULES}

As described in the chapter above, in the course of the experiments, the observed blockage events turned out to be extremely short (see Figure 4.30) compared to blockages by antibiotics described in literature for different porins,[52,53,55] making an analysis with common methods impossible. Furthermore, the recorded signal is always smoothened by the fourpole low pass filter applied during the measurements (see Equation 3.9). At an applied cutoff frequency of $f_{\mathrm{c}}=5 \mathrm{kHz}$, the filter rise time is $t_{\mathrm{R}}=70 \mu \mathrm{s}$. For signal durations in this range, the amplitudes and residence times are smoothened so strongly by the filter that the values determined during analysis appear lower than the original signal without filter, thus impeding a correct data evaluation.

Instead, the analysis routine JULES developed by Florian Pein (Munk group, Institute for Mathematical Stochastics, University of Göttingen) was used for data evaluation (see Chapter 3.6.4.2).[110] This routine enables model-free idealizations of events below the filter length by combining multiresolution techniques and local deconvolution. It detects all events of at least $80 \mu$ s duration reliably, reconstructs the original signals (see Figure 3.18 B) and gives the values for amplitude and residence time.

The conductivity amplitudes blocked by ampicillin are plotted as a histogram and a mean blockage amplitude is determined. Subsequently, the mean residence time of ampicillin in the pore and the frequency of blockage are calculated as characteristics of the ampicillin binding. For the determination of the mean residence times, a maximization of their corresponding likelihood is performed, assuming an exponential decay and a missing of events below $80 \mu \mathrm{s}$. This way, residence times shorter than the filter rise time can be determined. For the blockage frequencies, the mean time between two blockage events is multiplied with the number of open channels and the probability that an event is not missed. The inverse of this rescaled time is the blockage frequency.

As some questions concerning the interaction of ampicillin with PorB cannot be answered using electrophysiology alone, molecular dynamics (MD) simulations performed by Salomé Llabrés and Ulrich Zachariae (Computational Biology, School of Life Sciences, University of Dundee, UK) were used as supporting method (see Chapter 3.7). Docking calculations were used to study the binding mode of ampicillin and the permeation of ampicillin through the eyelet of PorB was explored via steered molecular dynamics simulations. Computational electrophysiology simulations were performed to investigate the influence of an applied voltage on the system. 
Most of the results of this chapter concerning PorB wt are accessible as preprint on the Biorxiv server (https://www.biorxiv.org/content/early/2018/04/18/303891).[156]

\subsubsection{Blockage amplitude}

The first parameter analyzed by JULES is the amplitude of the blockage caused by ampicillin. After signal reconstruction, a histogram of all fitted events can be plotted. Before the addition of antibiotic, only one conductance state can be found (Figure $4.32 \mathrm{C}$ ), corresponding to the open-channel-conductivity. In the presence of ampicillin, a second conductance state occurs (Figure 4.32 D), representing the conductance level of the blocked channel. The difference between these levels is the amplitude of conductivity blocked by the antibiotic (blocked amplitude). The blocked amplitude can be plotted as an event histogram including a kernel density estimation using a Gaussian kernel (Figure 4.32 E). The fit of a Gaussian distribution allows the determination of the mean blocked amplitude and thus to find out how many monomers are blocked. Indicated errors are the full width at half maximum (FWHM) of the Gauss fit.
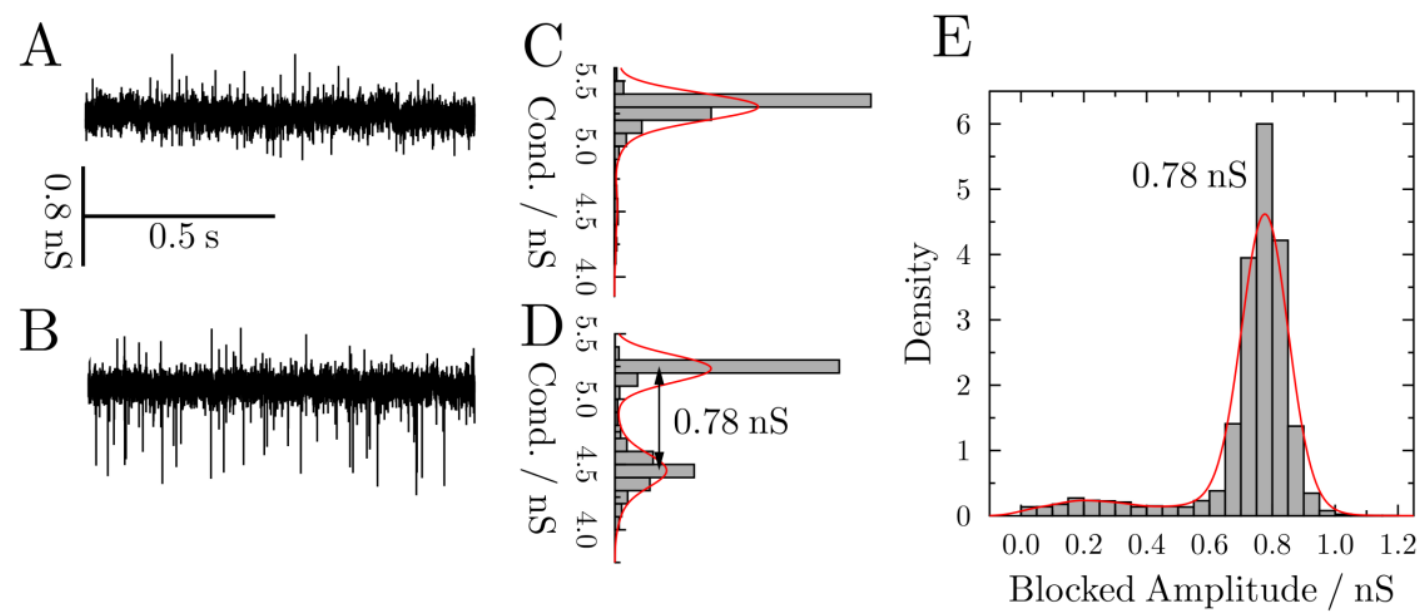

Figure 4.32. Evaluation of the experiments in presence of ampicillin. Exemplary conductivity traces recorded of PorB G103K at $+80 \mathrm{mV}$ in the absence (A) and presence of $1 \mathrm{mM}$ ampicillin (B) and corresponding histograms of all fitted conductance levels ( $C$ and $D$ ). The difference between the conductance levels found after antibiotic addition is the blocked amplitude and can be plotted as an event histogram (E) to determine the mean amplitude by a Gauss fit. The measuring buffer contained $1 \mathrm{M} \mathrm{KCl}, 10 \mathrm{mM}$ HEPES, pH 7.5 and the bilayers were composed of DPhPC/Chol 9:1.

Histograms of the amplitude blocked by ampicillin are presented in Figure 4.33 in the absence and presence of $1 \mathrm{mM}$ antibiotic. The histograms of the traces without ampicillin (A, C, E) demonstrate that the proteins themselves show fluctuations of the open-channel-conductivity. For PorB wt and G103K, the events detected in the absence of ampicillin are at a voltage of $+80 \mathrm{mV}$ about $4 \%$ of the number found in the presence of the antibiotic. The determined amplitude maxima of the blank samples are $A_{\mathrm{B}, \mathrm{WT},+80 \mathrm{mV}}=(0.35 \pm 0.57 \mathrm{nS})$ and 
$A_{\mathrm{B}, \mathrm{G} 103 \mathrm{~K},+80 \mathrm{mV}}=(0.34 \pm 0.13 \mathrm{nS})$. Values in this range can also be found in the presence of ampicillin (B and D). They have a low density compared to the maxima occurring only with antibiotic. The latter can be assigned to a blockage by ampicillin and are determined as $A_{\mathrm{WT},+80 \mathrm{mV}}=(1.18 \pm 0.10 \mathrm{nS})$ and $A_{\mathrm{G} 103 \mathrm{~K},+80 \mathrm{mV}}=(0.80 \pm 0.10)$.
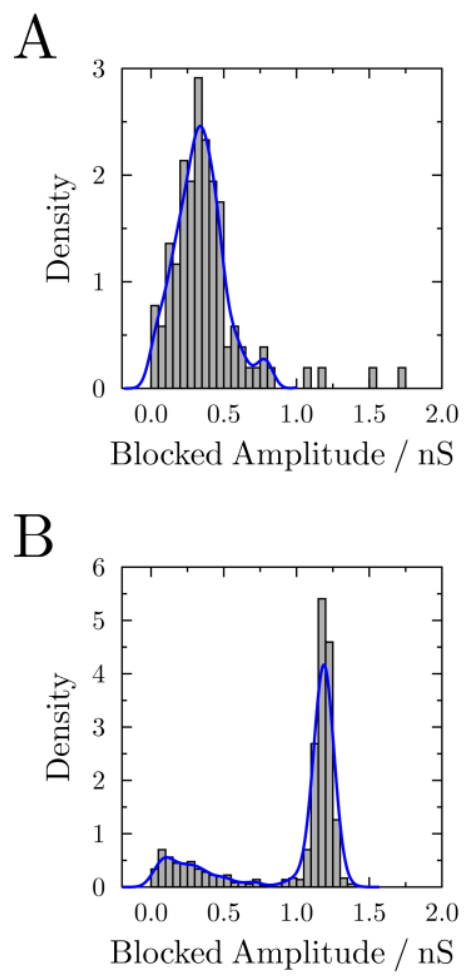

C

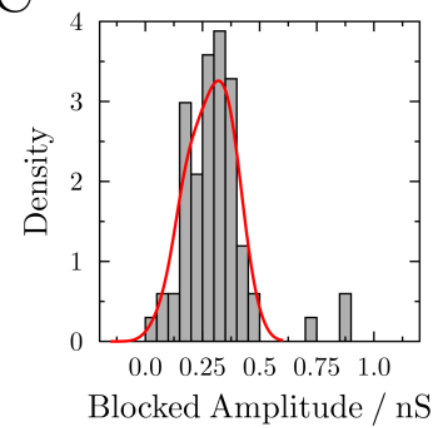

$\mathrm{D}$

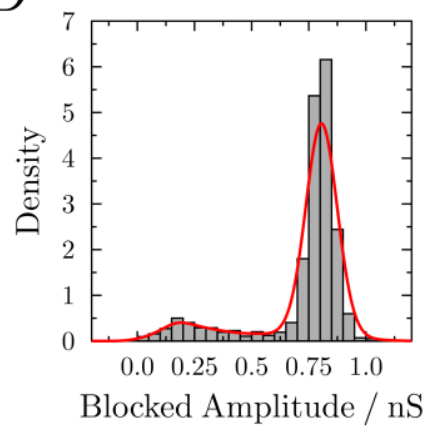

$\mathrm{E}$

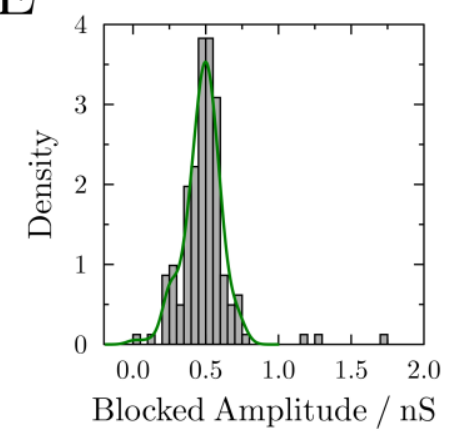

$\mathrm{F}$

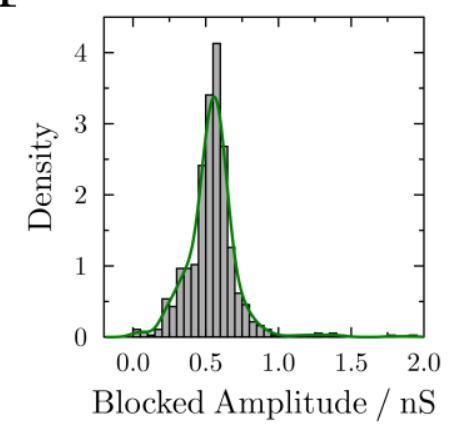

Figure 4.33. Amplitude event histograms of PorB wt (A and B), G103K (C and D) and G103D (E and F) in the absence (A, C, E) and presence of $1 \mathrm{mM}$ ampicillin (B, D, E). Blue, red and green lines are the kernel density estimations using a Gaussian kernel. For PorB wt and G103K the amplitude of the ampicillin blockage could be deter-

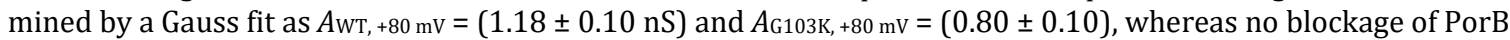
G103D by ampicillin was detected. Recordings were performed at $+80 \mathrm{mV}$ and $\mathrm{pH} 7.5$. The measuring buffer contained $1 \mathrm{M} \mathrm{KCl}, 10 \mathrm{mM}$ HEPES and the bilayers were composed of DPhPC/Chol 9:1.

For PorB G103D the situation is different. The histograms before (E) and after (F) addition of ampicillin are nearly identical with only one maximum at $A_{\mathrm{B}, \mathrm{G} 103 \mathrm{D},+80 \mathrm{mV}}=(0.50 \pm 0.28 \mathrm{nS})$ and $A_{\mathrm{G} 103 \mathrm{D},+80 \mathrm{mV}}=(0.57 \pm 0.27 \mathrm{nS})$, respectively. The number of detected events in the presence of ampicillin was never higher than the one before addition. Hence, the antibiotic does not interact with the mutant G103D, or at least not in a way that blocks the open channel conductance. The higher amplitude compared to the blank samples of PorB wt and G103K can be explained by the increased open-channel-noise of G103D (compare Chapter 4.2.2.4).

This increased noise was discussed to be caused by electrostatic repulsions between negatively charged residues at loop3 (see Chapter 4.2.2.5). Even if they do not lead to a large movement within loop3, such repulsions might destabilize the antibiotic binding site. The raised movement of single residues could hamper the interaction of the pores with ampicillin and prevent binding. 
In the following, only PorB wild type and the mutant G103K were investigated to characterize the interaction with ampicillin.

Amplitude event histograms of PorB wt and G103K in the absence and presence of $1 \mathrm{mM}$ ampicillin were generated at voltages between +40 and $+120 \mathrm{mV}$. At $+120 \mathrm{mV}$ the pores are often not open for a long time, therefore measurements at this voltage were rarely possible. The amplitude histograms of PorB wt (A and B) and G103K (C and D) at increasing voltage before and after ampicillin addition are shown in Figure 4.34.
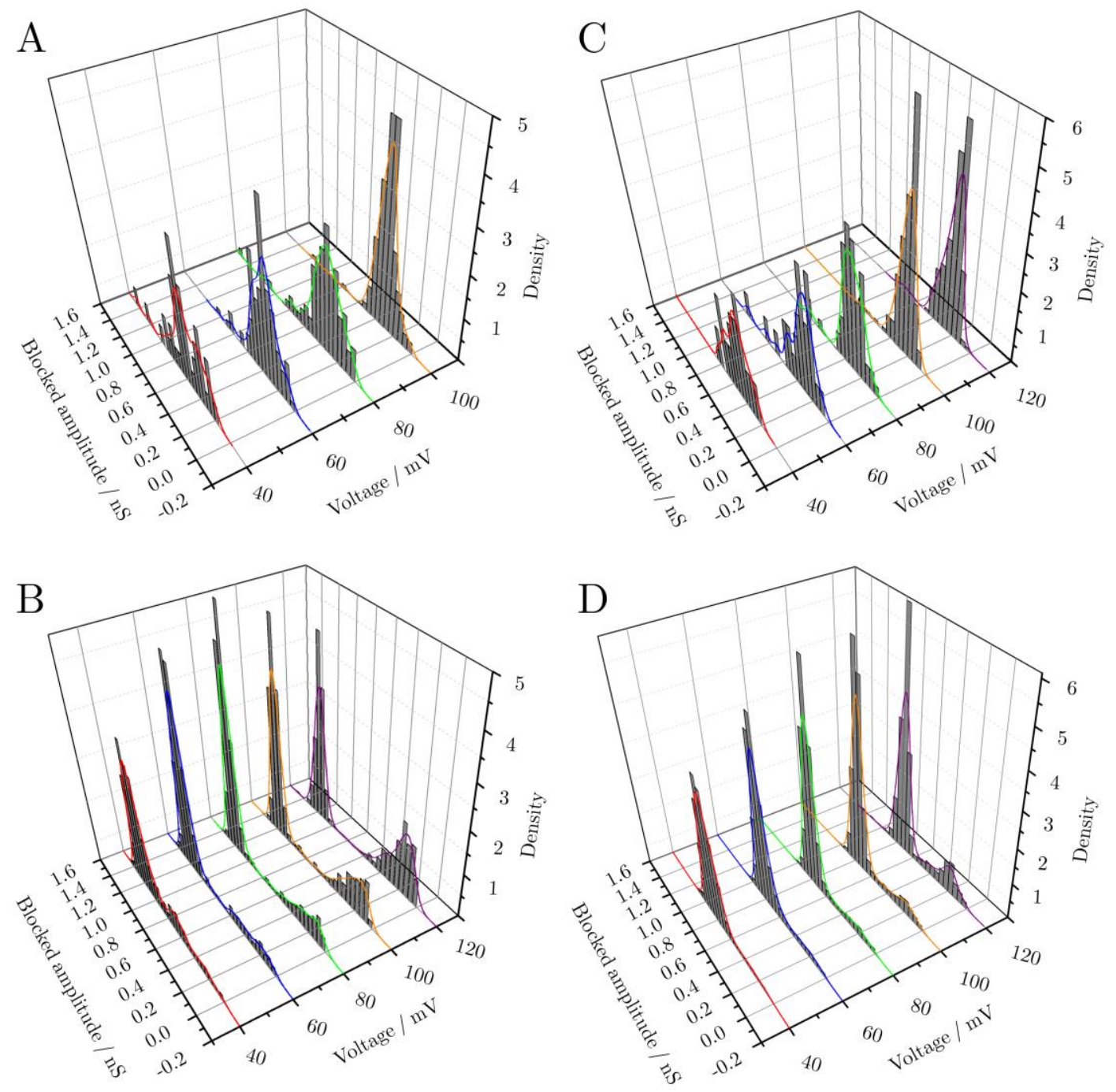

Figure 4.34. Voltage dependent amplitude event histograms of PorB wt ( $A$ and $B$ ) and G103K (C and D) in absence (A and C) and presence of $1 \mathrm{mM}$ ampicillin (B and D). The fits are kernel density estimations using a Gaussian kernel. Recordings were performed at $\mathrm{pH}$ 7.5. The measuring buffer contained $1 \mathrm{M} \mathrm{KCl,} 10 \mathrm{mM}$ HEPES and the bilayers were composed of DPhPC/Chol 9:1. 
In the absence of ampicillin (A and $\mathrm{C}$ ), the distribution of events is relatively broad, but the maxima are for both proteins similar to the values determined at $+80 \mathrm{mV}$ $\left(A_{\mathrm{B}, \mathrm{WT},+80 \mathrm{mV}}=(0.35 \pm 0.57 \mathrm{nS})\right.$ and $\left.A_{\mathrm{B}, \mathrm{G} 103 \mathrm{~K},+80 \mathrm{mV}}=(0.34 \pm 0.13 \mathrm{nS})\right)$ for all voltages above $+40 \mathrm{mV}$. The number of detected events increases with the voltage. At voltages between +40 and $+80 \mathrm{mV}$ the number of events detected in the blank measurements are about $4 \%$ of the ones found with ampicillin. This percentage increases to $10 \%$ at $+100 \mathrm{mV}$ and about $25 \%$ at $+120 \mathrm{mV}$, implying that the fluctuations of the open-channel-conductivity increase at higher voltages.

Open channel fluctuations are discussed to be caused by conformational changes of the channel.[183,184] Conformational changes of pores are in turn described to depend on the applied voltage (see Chapter 4.2.2.5).[161,162] Thus, it is not surprising that the open channel fluctuations of PorB vary with the applied voltage. Moreover, the destabilizing effect of an electric field on membranes, starting with increased fluctuations and formation of defects through electric breakdown is widely known. ${ }^{[185-189]}$ Stronger bilayer fluctuations at higher voltages are thus a second source of the increased number of events detected in the blank measurements.

The maximum found in the measurements in absence of ampicillin appears as well in the experiments with $1 \mathrm{mM}$ ampicillin (Figure $4.33 \mathrm{~B}$ and D) and its density also increases with the voltage. Consequently, it is not assigned to the influence of ampicillin. The second maximum not present in the histograms of the blank measurements represents the amplitude of the blockage caused by the ampicillin binding. The value slightly decreases with increasing applied voltage for both proteins (see Figure 4.35). For the wild type, the blocked amplitude decreases from $A_{\mathrm{WT},+40 \mathrm{mV}}=(1.20 \pm 0.02) \mathrm{nS}$ at $+40 \mathrm{mV}$ to $A_{\mathrm{WT},+120 \mathrm{mV}}=(1.16 \pm 0.02) \mathrm{nS}$ at $+120 \mathrm{mV}$. For the mutant, a decrease from $A_{\mathrm{G} 103 \mathrm{~K},+40 \mathrm{mV}}=(0.84 \pm 0.02) \mathrm{nS}$ at $+40 \mathrm{mV}$ to $A_{\mathrm{G} 103 \mathrm{~K},+120 \mathrm{mV}}=(0.75 \pm 0.01) \mathrm{nS}$ at $+120 \mathrm{mV}$ is detected. A comparable tendency was already found for the open channel conductivities in absence of ampicillin (compare Figure 4.19 and Figure 4.22). The discussed field-induced conformational changes of the pores (see Chapter 4.2.2.5) might influence the binding between pore and antibiotic and lead to a slightly altered position of the bound ampicillin. A shift of the antibiotic's position would lead to a more or less effective blockage of the pore and thus a varying blockage amplitude. 


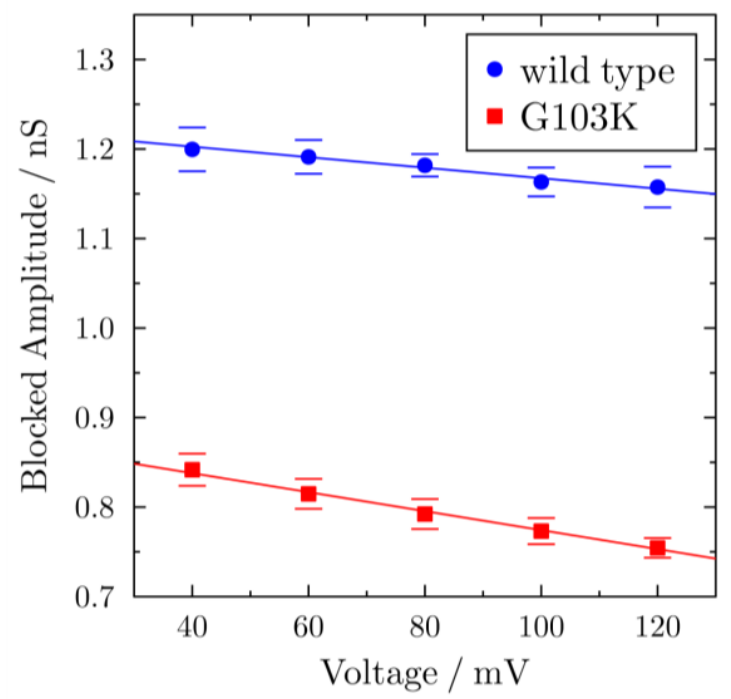

Figure 4.35. Amplitudes blocked by ampicillin (1 mM) at increasing voltages for PorB wt (blue) and G103K (red). Four values were averaged and the error is the standard deviation. For both proteins, a decrease of the amplitudes with increasing voltage is visible. The measuring buffer contained $1 \mathrm{M} \mathrm{KCl}, 10 \mathrm{mM} \mathrm{HEPES}, \mathrm{pH} 7.5$ and the bilayers were composed of DPhPC/Chol 9:1.

Blocked amplitudes are only compared to the pore conductances obtained in solvent-free membranes. The values obtained in BLMs are only based on one measurement, respectively, and are thus less reliable. For the mutant, the trimer conductivity was determined as $G_{\mathrm{G} 103 \mathrm{~K}, \mathrm{~T}}=(0.73 \pm 0.14 \mathrm{nS})$ at an applied voltage of $+40 \mathrm{mV}$. The determined blocked amplitude of $A_{\mathrm{G} 103 \mathrm{~K},+40 \mathrm{mV}}=(0.84 \pm 0.02) \mathrm{nS}$ thus corresponds to a fully blocked trimer. In case of the wild type, the blocked amplitude $A_{\mathrm{WT},+40 \mathrm{mV}}=(1.20 \pm 0.02) \mathrm{nS}$ is between the pore conductivity $G_{\mathrm{WT}, \mathrm{T}}=(1.48 \pm 0.46 \mathrm{nS})$ of the trimer and the conductivity $G_{\mathrm{WT}, \mathrm{D}}=(0.84 \pm 0.26 \mathrm{nS})$ of the dimer. Consequently, more than two monomers are blocked, but the amplitude does not match a complete blockage of the trimer. A reasonable explanation for this discrepancy is that in each monomer the ion flux is not completely blocked due to the interaction with ampicillin and a small leak current remains per monomer. Finally, it can be assumed that for both porins all three monomers are blocked upon interaction with ampicillin.

The influence of the ampicillin concentration on the blocked amplitude was investigated as well. Measurements were performed at $+80 \mathrm{mV}$ under addition of ampicillin concentrations increasing from 0.1 to $1.0 \mathrm{mM}$. The amplitude event histograms for the wild type $(\mathrm{A})$ and the mutant G103K (B) are shown in Figure 4.36. Especially at $0.1 \mathrm{mM}$, but as well at $0.2 \mathrm{mM}$ antibiotic, the maximum assigned to fluctuations of the open-channel-conductivity (about $0.3 \mathrm{nS}$, compare Figure $4.34 \mathrm{~A}$ and $\mathrm{C}$ ) accounts for a large number of the detected events. At higher concentrations, the peak corresponding to the blockage by ampicillin is dominant. 
The blocked amplitude averaged over all concentrations including the standard deviation is $A_{\mathrm{WT}}=(1.19 \pm 0.01 \mathrm{nS})$ for the wild type and $A_{\mathrm{G} 103 \mathrm{~K}}=(0.81 \pm 0.01)$ for the mutant. Thus, even at very low ampicillin concentrations the blockage amplitude corresponds to the conductivity of three monomers for both proteins.
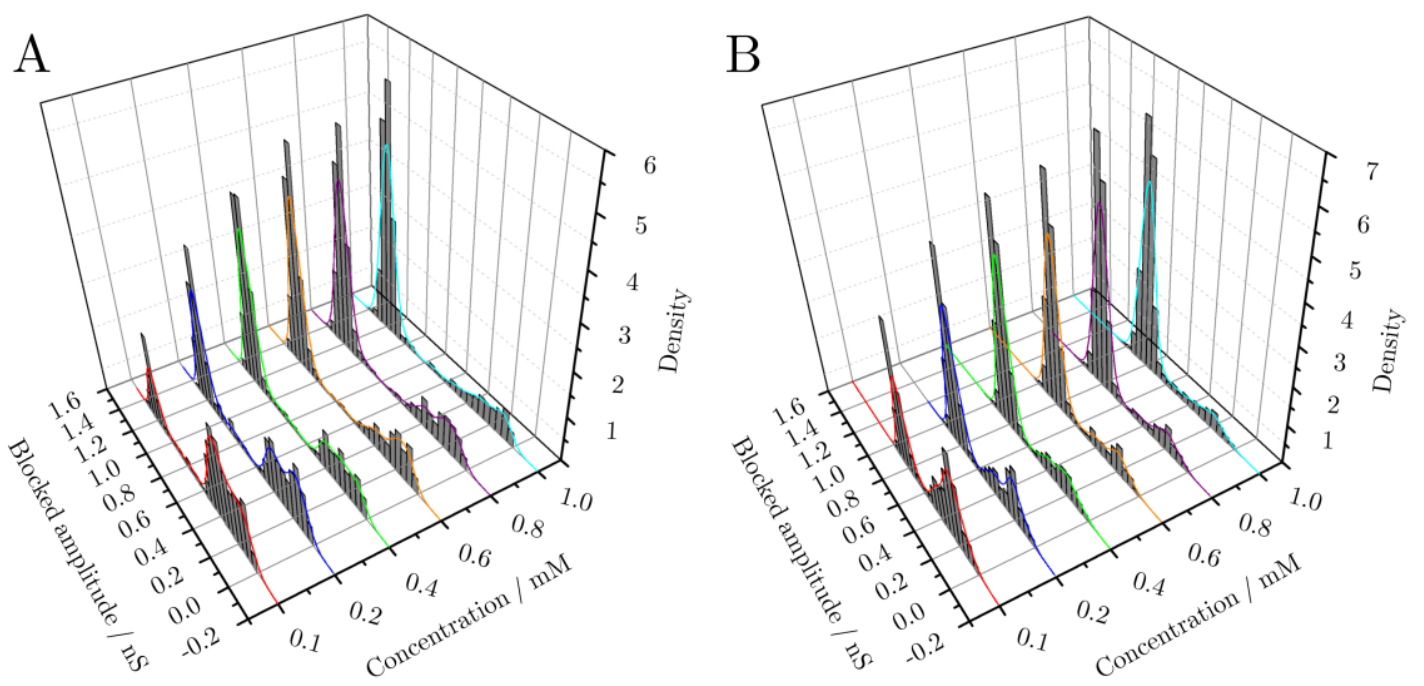

Figure 4.36. Amplitude event histograms of PorB wt $(A)^{[156]}$ and G103K (B) for increasing ampicillin concentrations. The fits are and kernel density estimations using a Gaussian kernel. With increasing concentration, the peak corresponding to the blockage by ampicillin becomes more dominant, whereas the value of the blocked amplitude is not influenced. Recordings were performed at $+80 \mathrm{mV}$ and $\mathrm{pH} 7.5$ The measuring buffer contained $1 \mathrm{M} \mathrm{KCl}$, $10 \mathrm{mM}$ HEPES and the bilayers were composed of DPhPC/Chol 9:1.

The fact that the amplitude of blockage caused by ampicillin matches the trimer conductivity for both proteins at all voltages and concentrations can be interpreted in two ways: Either all three monomers are simultaneously blocked by three antibiotic molecules, or the blockage of one monomer results in a cooperative closure of the other two. A simultaneous blockage of a whole porin trimer is rather unusual. Mostly, independent blockage of single monomers is reported, concerning the interaction with antibiotics ${ }^{[52,55]}$ and the transport of sugars through maltoporin.[51,190] Regarding the binding of sugars to chitoporin, a cooperative behavior between the monomers was found.[191] A blocked monomer lead to an increased trapping rate for the other two. However, this effect is apparently induced by a rapid re-trapping of recently escaped molecules.

For a cooperative effect, binding of an antibiotic to one monomer has to change the conformation of the other two. Two effects are possible: The non-blocked monomers could adapt a conformation more favorable for antibiotic binding or undergo larger conformational changes resulting in a complete closure. Both scenarios would lead to a loss of conductivity equal to a whole trimer, as detected for PorB in this study. Electrophysiological data alone cannot show which scenario is more likely. 


\subsubsection{Unilateral interaction and sign of the applied voltage}

\section{Influence of the side of ampicillin addition}

Ampicillin blocks the PorB channel only when added to its extracellular side. Together with the asymmetric location of the binding site, this suggests a high energy barrier for ampicillin entering from the periplasmic side.

In most experiments, ampicillin was added to both sides of the bilayer. Surprisingly, no pore blockage, neither of the wild type nor of the mutant, was observed when ampicillin (up to $8 \mathrm{mM}$ ) was only added to the trans side, i.e., the side opposed to protein addition. The analysis using the JULES routine revealed no increase of detected events without and after addition of $8 \mathrm{mM}$ ampicillin. Consequently, the insertion of PorB happens completely unidirectional and ampicillin causes channel blockage only when entering from the side of protein addition. A unidirectional insertion is known from other porins as well.[101,190,192] In this process, the application of a voltage seems to be essential,[57] but the sign of the applied voltage is found to have no effect on the orientation of the pore.[57,192,193] The same observation was made for PorB in this work: insertions at $+40 \mathrm{mV}$ and $-40 \mathrm{mV}$ both lead to an unidirectional insertion of PorB. Thus, an influence of the protein's dipole moment on its orientation in the membrane, as hypothesized for $\beta$-barrels by Vasanthi and Krishnaswamy, ${ }^{[194,195]}$ can be excluded. The most important effect on the insertion direction described in literature for $\beta$-barrels is the structural asymmetry between periplasmic and extracellular side. An influence of the pore structure on the insertion mechanism is described for $\mathrm{OmpF}{ }^{[193]}$ and maltoporin, whereas for the latter indications of an insertion with the extracellular loops first, ${ }^{[57,190]}$ as well as the opposite direction are described.[101,196] PorB, OmpF and maltoporin show notable structural similarities: They possess many extracellular loops, while the periplasmic side shows only short turns. ${ }^{[35,197]}$ This suggests that PorB insertion is influenced by its structure.

A strong dependence of the blockage frequency on the side of antibiotic addition is also described in literature, $[53,55]$ but a complete unilateral interaction with antibiotics is not known for any porin so far. In this context, an asymmetry of the antibiotic binding site concerning the energy barriers for entering from the extracellular and periplasmic side of the pore is discussed. In case of PorB, this would hint towards a strongly pronounced asymmetry of the constriction region, leading to an extremely high energy barrier for entering from and leaving towards the trans side, raising the question if a transport of ampicillin is even possible. 
Using anionic and zwitterionic ampicillin, docking calculations were performed in PorB wt and PorB G103K and the resulting binding modes were validated via independent MD simulations. This approach resulted in a single, asymmetric binding site of ampicillin, located at the base of the extracellular (EC) side of the constriction zone (see Figure 4.37A; Salomé Llabrés and Ulrich Zachariae, Computational Biology, School of Life Sciences, University of Dundee, UK, unpublished results). At this position, the antibiotic - zwitterionic, as well as anionic species - obstructs the entrance of the constriction region to a large extent,[156] which is in good agreement with the blockage of the open channel conductivity observed in the patch-clamp measurements.

In PorB wt ( Figure $4.37 \mathrm{~B}$, green sticks), the amino group of ampicillin interacts with the sidechain of E116 by the formation of a salt bridge and additionally with the backbone of G112 via its carbonyl group. The backbones of W109, E110, S111, W115, G120 and E116, together with the aliphatic parts of the E116 and E110 side chains, form a shallow hydrophobic site, to which the phenyl group of ampicillin is bound. A further salt bridge is formed between the carboxyl group of ampicillin and the residues K64 and R77. In the mutant G103K, a similar binding mode was found (Figure 4.37 B, cyan sticks; Salomé Llabrés and Ulrich Zachariae, Computational Biology, School of Life Sciences, University of Dundee, UK, unpublished results). The interactions between the amino group and residues E116 and G112, between the phenyl moiety and the hydrophobic cavity, as well as the salt bridge between R77 and the acid moiety are conserved. Additionally, a hydrogen bond is formed between the mutated lysine residue (K103, orange sticks) and the carbonyl group of the $\beta$-lactam ring of ampicillin. 

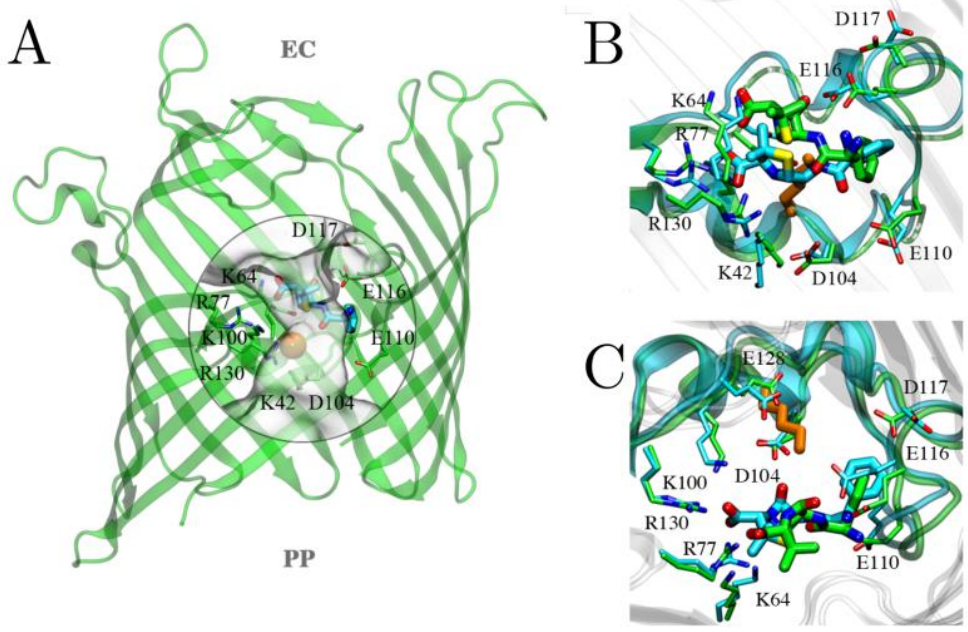

Figure 4.37. Location of the ampicillin binding site. Ampicillin binds at the extracellular (EC) side of the PorB constriction zone (transparent surface) (A). Carbon atoms of PorB are presented as green sticks, carbon atoms of ampicillin as cyan sticks. The residue 103 is shown as orange sphere. Binding mode of ampicillin, viewed from the membrane side (B) and the extracellular side (C). PorB wt is shown in green, the mutant G103K in cyan and the mutated residue as orange stick. (Salomé Llabrés and Ulrich Zachariae, Computational Biology, School of Life Sciences, University of Dundee, UK, unpublished results).

The asymmetric location of the binding site at the extracellular side together with the absence of blockage events when ampicillin is added to the trans side could indicate that the binding site is not accessible from the periplasmic side of PorB. At least, the presence of a high energy barrier for permeation from the periplasmic to the extracellular side is very likely, although the actual energy barriers could not be calculated via MD simulations.

Further, these findings allow conclusions concerning the insertion direction of PorB. If the ampicillin binding site is only accessible from the extracellular side and only cis-side-addition of ampicillin leads to a binding, PorB has to insert with the periplasmic side first, so that the extracellular side is exposed to the cis side of the bilayer. Considering the increased steric hindrance of the extracellular loops compared to the short periplasmic turns, this way of insertion is probably energetically favored.

Two scenarios would explain an absence of blockage events although a binding of ampicillin happens upon trans side addition.

The first concerns the orientation of ampicillin at the binding site. Crystal structures of OmpF and several antibiotics demonstrated that not all of them are bound perpendicular to the pore axis. Carbenicillin is oriented parallel to the channel wall and does not bind directly at the constriction zone.[15] Molecular dynamics simulations showed that this antibiotic does not block the ion flux through the pore when bound to OmpF. Such a situation could explain the absence of blockage events when ampicillin is added to the trans side. Ampicillin would have 
to adapt a parallel orientation when entering from the periplasmic side of PorB and a perpendicular orientation when entering from the extracellular side.

Different ampicillin residence times upon addition to the extracellular or periplasmic side of PorB could explain the absence of blockage events upon addition to the trans side. The residence times upon addition to the cis side (extracellular entrance of PorB) are already very short. If the residence times were even shorter upon addition to the trans side (periplasmic entrance of PorB), they would be too short to detect a blockage of the channel with a time resolution of $70 \mu$ s set by the filter.

However, both scenarios would require two separate binding sites at the periplasmic and extracellular side of PorB. As only one binding site was found during the docking calculations, they can be excluded.

The question if a translocation of ampicillin through PorB takes place could not be answered neither from the electrophysiological measurements nor by the MD simulations. The observed blockage of the ion flux only confirms an interaction between antibiotic and pore, but it cannot be distinguished between a translocation and a release to the same side after binding. The conducted MD simulations could not detect any translocation of ampicillin through PorB, because it would only be a rare event occurring at longer time-scales than the one of the simulations. Liposome swelling assays performed by Christof Kattner (ZIK HALOmem, Membrane Protein Biochemistry, Martin-Luther-University Halle-Wittenberg) indicate that a transport of ampicillin through PorB wt and PorB G103K is possible.[149] The addition of PorB containing liposomes to an isoosmotic buffer solution containing $15 \mathrm{mM}$ ampicillin lead to a decrease in optical density caused by the influx of antibiotic and water (concentration balance), followed by a rupture of the liposomes. Control experiments with protein-free liposomes showed no decrease of the optical density. However, the experimental conditions are different compared to the electrophysiological measurements. Especially the absence of a transmembrane potential might influence the results, considering the versatile impact of an applied voltage on the pore insertion and function (see above and Chapter 4.2.2.5). As it cannot be assured that a transport of ampicillin takes place in the patch-clamp experiments of this project, the interaction of ampicillin with PorB will be only discussed in terms of binding.

\section{Influence of the sign of the applied voltage}

The absence of blockage events at applied negative voltages can be explained by the direction of the electro-osmotic flow, which pushes the ampicillin molecules back from the binding site towards the extracellular entrance of PorB. 
Interestingly, blockage events only appeared at positive applied voltages, whereas at negative voltages up to a concentration of $8 \mathrm{mM}$ ampicillin no blockage was observed. An obvious explanation would be that at $\mathrm{pH} 7.5$ the percentage of anionic ampicillin molecules (compare Chapter 3.3) is forced into the pore by an applied positive voltage, while a negative voltage leads to an electrostatic repulsion. In order to test this theory, experiments with the dominantly zwitterionic ampicillin species at pH 6 were performed. As they led to the same result, electrostatic attraction and repulsion by the applied voltage are not responsible for the dependence on the sign of the voltage.

For OmpF and zwitterionic ampicillin,[52,56] as well as for Omp36 and anionic ertapenem ${ }^{[55]}$ the sign of the applied voltage was found to strongly influence the number of blockage events. However, a blockage occurring only at negative or positive voltages is not described in literature.

Therefore, the influence of the applied voltage was investigated for PorB wt via computational electrophysiology simulations.[156] Without antibiotic present, a similar trimer conductance was found at positive and negative voltages. In the presence of ampicillin, however, the channel conductance is strongly reduced at positive voltages, while at negative voltages the reduction is less pronounced. The reason was found in a destabilizing effect of the negative voltage on the ampicillin binding. Due to the pore diameter of PorB, ions can permeate partially hydrated. The co-permeation of water is termed electro-osmotic flow (EOF).[198] At positive voltages, the EOF in PorB occurs from the extracellular to the periplasmic side (see Figure 4.38 A). As a result, it drives present ampicillin molecules towards the eyelet of PorB (which presumably is only accessible from the extracellular side) and thus stabilizes the binding of ampicillin. At negative voltages, the direction of the EOF is inverted (see Figure 4.38 B). Consequently, it destabilizes the ampicillin binding, because the antibiotic molecules are pushed back from the binding site towards the extracellular entrance of the pore. This finding explains, why only at positive applied voltages a blockage of PorB due to binding of ampicillin is observed. Further, the strong influence of the EOF on ampicillin binding hints at a low binding affinity. 
A

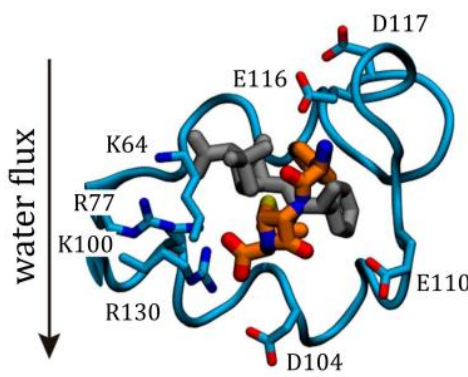

B

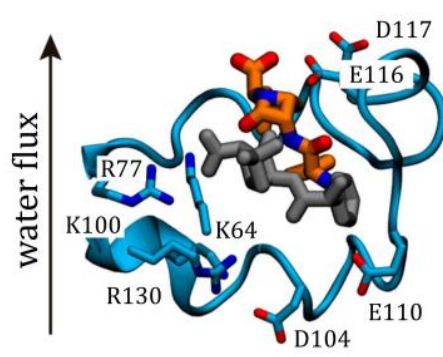

Figure 4.38. Direction of the electro-osmotic flow (EOF) and its effect on the ampicillin binding mode at positive (A) and negative (B) voltages. Loop3 of PorB wt is shown in blue, the initial position of ampicillin is represented in grey sticks and its final position influenced by the EOF is depicted as orange sticks.[156]

An additional reason for the absence of blockage events at negative voltages might be the voltage induced elastic deformations of pores described in Chapter 4.2.2.5. They include a variation of the pore size and the orientation of single residues. ${ }^{[161,162]}$ In those studies, a strong dependence especially on the sign of the applied voltage is reported. Such effects might influence the interaction between ampicillin and PorB as well, impeding ampicillin binding at negative voltages.

\subsubsection{Residence times of ampicillin}

The residence time is one characteristic of the interaction between an antibiotic and a pore. It represents the time the molecule is bound to the pore and thus blocking the ion current. A longer residence time can indicate a higher binding affinity, if $\mathrm{k}_{\mathrm{on}}$ is uncanged, which could be influenced by a point mutation near or at the binding site, because it might influence the geometry and the electrostatic surface. In case of the mutant G103K, both effects are possible, since a long residue and a positive charge are introduced. The mean residence time of ampicillin in PorB wt and G103K were determined dependent on the ampicillin concentration and the applied voltage, by exponential fitting of the blockage time histograms of all events assigned to the blockage amplitude of ampicillin (see Figure 3.18).

\section{Concentration dependence of residence times}

The residence times do not depend on the ampicillin concentration. The longer residence times obtained for the mutant are ascribed to a higher energy barrier due to an unfavorable orientation upon entering the constriction region. 
For the investigation of the concentration dependency of the residence times, increasing concentrations of ampicillin, ranging from 0.1 to $1.0 \mathrm{mM}$, were added to both measuring chambers at $\mathrm{pH}$ 7.5. The recordings were performed at $+80 \mathrm{mV}$, because at this voltage the signalto-noise ratio turned out to be best. A good signal-to-noise ratio is necessary to detect the relatively small number of events expected at low ampicillin concentrations.

Exemplary residence times for PorB wt (blue) ${ }^{[156]}$ and the mutant (red) are plotted in Figure 4.39, including upper and lower confidence intervals (CIs). Due to the small number of events, the values at $0.1 \mathrm{mM}$ and $0.2 \mathrm{mM}$ have a large error margin. Therefore, the linear fits (blue and red line) were carried out including the confidence intervals and show no notable dependence of the residence times on the ampicillin concertation. The residence times calculated using the weighted mean are $\tau_{\mathrm{R}, \mathrm{WT}}=(35 \pm 1) \mu \mathrm{s}$ for the wild type and $\tau_{\mathrm{R}, \mathrm{G} 103 \mathrm{~K}}=(44 \pm 1) \mu \mathrm{s}$ for the mutant.

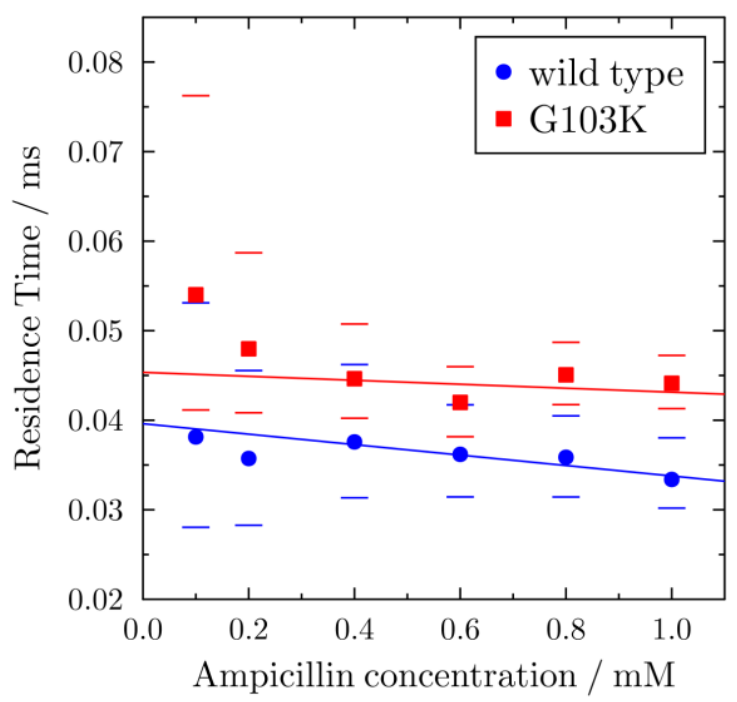

Figure 4.39. Exemplary residence times of ampicillin with confidence intervals (bars) for PorB wt (blue) ${ }^{[156]}$ and G103K (red) at increasing ampicillin concentrations. The linear fits (blue and red line) were carried out considering the CIs and show no notable concentration dependence. Recordings were performed at $+80 \mathrm{mV}$ and $\mathrm{pH}$ 7.5. The measuring buffer contained $1 \mathrm{M} \mathrm{KCl}, 10 \mathrm{mM}$ HEPES, pH 7.5 and the bilayers were composed of DPhPC/Chol 9:1.

Concentration independency of antibiotic residence times is known for other porins $[55,56,199]$ and indicate that the time an antibiotic molecule is bound to the pore is not influenced by the number of molecules available for binding, which is reasonable. The values determined for PorB and ampicillin are very small in comparison with literature [52,53,55], which could indicate a rather low binding affinity between antibiotic and pore, as already suggested by the strong influence of the EOF on the ampicillin binding (see Chapter 4.3.2.2).

The shorter residence times obtained for PorB wt compared to the mutant could imply a slightly lower binding affinity of ampicillin for the wild type. An influence of point mutations 
at the constriction region on the binding affinity of antibiotics was found for OmpF, caused by an alteration of the energy barriers. ${ }^{[53,200]}$ Molecular dynamics simulations of the translocation of antibiotics through various porins revealed the important role of the pore's inner electric field. During translocation, the molecule aligns its dipole moment according to this electric field, forcing it to reorient several times during the process. ${ }^{[63]}$ In case of antibiotics, the most significant reorientations happen upon entering and leaving the constriction region, making these areas particularly sensitive to mutations. Altering the electric field in these areas by a point mutation can lead to an unfavorable pre-orientation (orientation upon entering the constriction region) of the antibiotic, so that it has to reorient within the constriction zone in order to enable binding. Such a reorientation involves an increased energy barrier for translocation. This is illustrated by the two OmpC mutants OmpC20 and OmpC33.[119] The pre-orientation of the antibiotics is more pronounced in case of $\mathrm{OmpC} 33$, hampering a reorientation upon approaching the constriction zone. Further, the less ordered electric field of OmpC33 leads to a reduction of the driving force for reorientation. Consequently, the antibiotic molecules have the wrong orientation when entering the constriction zone, which happens more often for OmpC33 than for OmpC20. These molecules can adjust their orientation in one of two ways: They can either leave the constriction region, reorient and enter again, or reorient directly at the constriction region. The second possibility would mean a higher energy barrier for translocation, thus an increase of the antibiotic residence time, as observed in the case of PorB G103K. As this mutation was shown to alter the surface potential within the constriction region (see Figure 4.15), it likely changes the electric field as well. A result could be an unfavorable pre-orientation of ampicillin and possibly a reduced driving force for reorientation. The additional contact between the bound ampicillin and the mutant pore compared to the wild type pore (see Chapter 4.3.2.2) found in the MD simulations performed on PorB and the high steric hindrance of the lysine residue might further complicate the reorientation and thus increase the energy barrier for translocation, explaining the longer residence times in case of the mutant.

\section{Voltage dependence of residence times}

The voltage dependence of the ampicillin residence times is similar for both porins and is thus ascribed to voltage dependent pore conformations.

The voltage dependence of the ampicillin residence times was investigated at a fixed antibiotic concentration of $1 \mathrm{mM}$ (both chambers) increasing the applied voltage from +40 to 
$+120 \mathrm{mV}$. The experiments were carried out at a pH of 7.5 (60\% negatively charged ampicillin) and pH 6 (almost exclusively zwitterionic ampicillin) in order to find out which effects can be attributed to the nature of the antibiotic and which are properties of the porin itself. In Figure 4.40, the residence times for PorB wt (blue) ${ }^{[156]}$ and G103K (red) are plotted against the applied voltage, including a "guide-to-the-eye", which is a parabolic fit without any further meaning (blue and red lines). A similar voltage dependence is visible for both porins. At $\mathrm{pH} 7.5$, the residence times increase between +40 and $+80 \mathrm{mV}$, resulting in a maximum between +80 and $+100 \mathrm{mV}$ for the wild type and around $+100 \mathrm{mv}$ for the mutant. At $\mathrm{pH} 6$, the voltage dependence is nearly unchanged for the wild type with shorter residence times at low voltages. For the mutant the maximum residence time can be found at lower voltages compared to the situation at $\mathrm{pH} 7.5$ and the values are smaller at all voltages. In general, at all voltages and independent of the $\mathrm{pH}$, the residence times for the wild type are shorter than for the mutant.

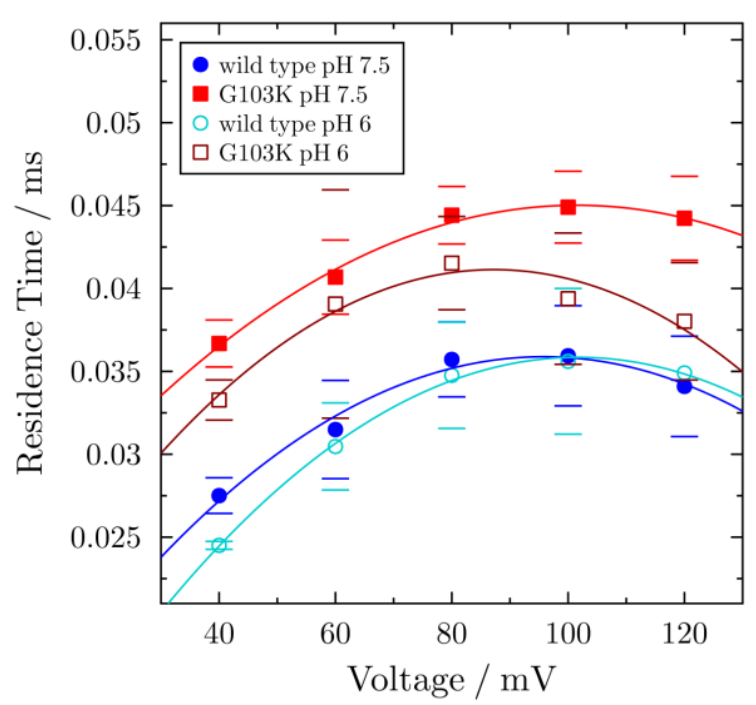

Figure 4.40. Voltage dependent residence times of ampicillin (1 mM) for PorB wt (blue)[156] and G103K (red) with "guide-to-the-eye" (lines). At pH 7.5 four and at pH 6 two experiments were averaged and the errors represent the standard deviation. For PorB wt at pH 6 and $+120 \mathrm{mV}$ only one value was available, so that no standard deviation can be indicated. The voltage dependence is similar for both proteins and both $\mathrm{pH}$ values. The residence times are shorter for the wild type than for the mutant. The measuring buffer contained $1 \mathrm{M} \mathrm{KCl}, 10 \mathrm{mM}$ HEPES, $\mathrm{pH}$ and the bilayers were composed of DPhPC/Chol 9:1.

Voltage dependent residence times are reported for antibiotics bound to porins,[52,55,56] as well as for the transport of sugars through maltoporin.[51] In this context, the alteration of the pore geometry due to the applied electric field is considered as probable cause.[51,160] The voltage dependence of the pore conformation of $\alpha$-hemolysin already described in Chapter 4.2.2.5 and 4.3.2.1 supports this idea. ${ }^{[161,162]}$ As binding between an antibiotic and the pore is based on an exact fit, it is affected by changes in geometry. Alterations of the binding affinity 
are in turn detectable by different residence times. The observation that the voltage dependence of the ampicillin residence times in PorB is neither affected by the single point mutation nor the charge of the antibiotic implies a major influence of the proteins' overall structure. Consequently, conformational changes play a major role, whereas no impact of an attraction of the charged ampicillin by the applied positive voltage is detectable.

Independent of the applied voltages, the residence times obtained for the mutant PorB G103K are longer compared to those for the wild type, as already found for varying ampicillin concentrations (see discussion above). Here again, the charge of ampicillin is not crucial, because this effect is the same for the partly anionic molecule at pH 7.5 and the zwitterionic species at $\mathrm{pH} 6$.

The longer residence times for the partly anionic compared to the zwitterionic ampicillin are only distinct in case of the mutant and hint at an increased energy barrier for the charged molecule. An explanation can again be found considering the dipole alignment.[63] The dipole of a charged molecule differs from the one of its zwitterionic form, thus the direction of the alignment within the pore's electric field is altered. Apparently, the anionic ampicillin is forced to adapt a less favorable pre-orientation than the zwitterionic species and needs to reorient more strongly within the constriction zone. As a result, it faces a higher energy barrier and resides longer in the pore. The alteration of the pore's inner electric field and the increased steric hindrance due to the point mutation seem to cause this effect.

\subsubsection{Blockage frequencies of ampicillin}

A second characteristic of antibiotic binding is the frequency of blockage, i.e., how often the molecule interacts with the pore. Blockage frequencies were determined by exponential fitting of the histograms of the times between two blockage events (see Chapter 3.6.4.2). The shorter the residence time, the less events are detectable due to the time resolution limit. As described in Chapter 4.3.2.3, residence times vary with the applied voltage and are different for the wild type than for the mutant. This would influence the calculated blockage frequencies. Therefore, the analysis routine takes into account missed events to enable a correct determination of the frequencies. The obtained values are divided by the probability that an event is not missed. This probability is generated by simulations. The number of events depends on the pores available for blockage and is thus proportional to the conductivity. In order to compare measurements with different numbers of inserted trimers and the values of wild type and the mutant, which have different open channel conductivities, the frequencies 
were normalized to the number of trimers. Blockage frequencies were investigated dependent on the ampicillin concentration and the applied voltage.

\section{Concentration dependence of blockage frequencies}

For the determination of the concentration dependent blockage frequencies, the same measurements were analyzed as for the concentration dependent residence times. The ampicillin concentration was increased from 0.1 to $1.0 \mathrm{mM}$ at $+80 \mathrm{mV}$ and $\mathrm{pH} 7.5$.

Exemplary frequencies obtained for the wild type (blue) ${ }^{[156]}$ and the mutant (red) are shown in Figure 4.41. At $0.1 \mathrm{mM}$ ampicillin, the frequency is near zero for both and increases linearly with the concentration. Linearly increasing blockage frequencies are in good agreement with literature ${ }^{[55,56,199]}$ and can be rationalized by an increasing number of molecules available for binding.

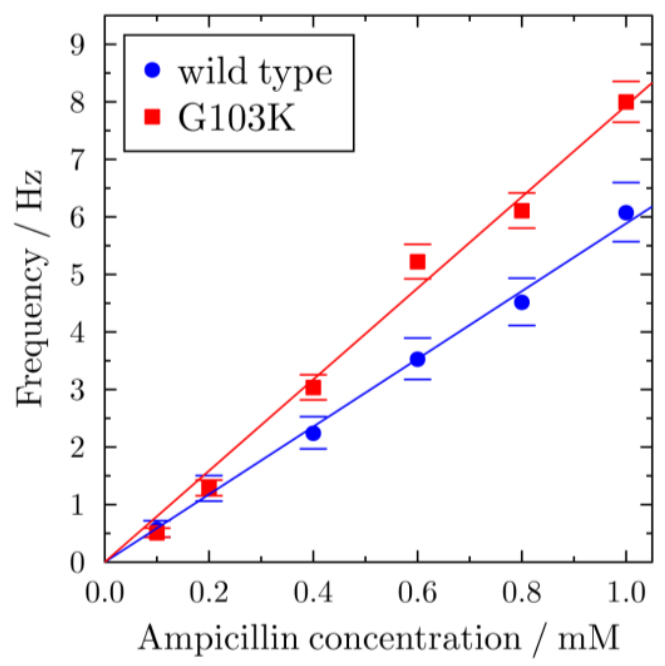

Figure 4.41. Exemplary concentration dependent blockage frequencies for PorB wt (blue) ${ }^{[156]}$ and G103K (red) with confidence intervals (bars). Measurements were performed at $\mathrm{pH} 7.5$ and an applied voltage of $+80 \mathrm{mV}$. A linear increase of the frequencies with increasing ampicillin concentration is visible for both porins. The measuring buffer contained $1 \mathrm{M} \mathrm{KCl}, 10 \mathrm{mM}$ HEPES, $\mathrm{pH}$ and the bilayers were composed of DPhPC/Chol 9:1.

\section{Voltage dependence of blockage frequency}

The opposed voltage dependence of the blockage frequencies of wild type and mutant are caused by different orientations while traversing the constriction region. The higher blockage frequencies for the mutant indicate a higher level of same-side release due to an unfavorable orientation upon entering the constriction region.

Blockage frequencies were as well investigated dependent on the applied voltage. In order to check for the influence of the negative charge of ampicillin, measurements at $\mathrm{pH} 7.5$ and $\mathrm{pH}$ 6 in the presence of $1 \mathrm{mM}$ ampicillin were evaluated. The resulting frequencies are plotted in 
Figure 4.42. A linear increase with increasing voltage can be observed for the mutant G103K (red) at both $\mathrm{pH}$ values. At pH 7.5, the frequencies at low voltages are lower than at $\mathrm{pH} 6$, but show a steeper slope, leading to similar values at high voltages. Opposing tendencies are found for the wild type (blue) ${ }^{[156]}$ : The blockage frequencies decrease linearly with increasing voltage, very strongly at $\mathrm{pH} 6$ and only slightly at pH 7.5. Higher values are observed for low voltages at $\mathrm{pH}$ 6, but due to the steep slope, the frequencies are similar for both $\mathrm{pH}$ values at high voltages.

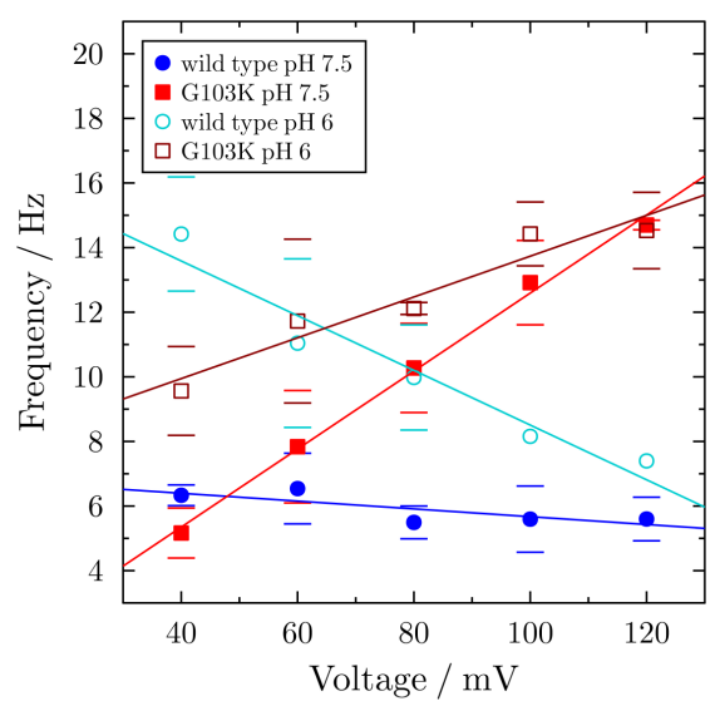

Figure 4.42. Voltage dependent blockage frequencies for PorB wild type (blue) ${ }^{[156]}$ and the mutant G103K (red) in the presence of $1 \mathrm{mM}$ ampicillin. At pH 7.5 four and at pH 6 two measurements were averaged and the errors represent the standard deviation. In case of PorB wt at pH 6, only one value was available at +100 and $+120 \mathrm{mV}$, thus no standard deviation can be indicated. The measuring buffer contained $1 \mathrm{M} \mathrm{KCl,} 10 \mathrm{mM} \mathrm{HEPES}, \mathrm{pH}$ and the bilayers were composed of DPhPC/Chol 9:1.

In literature, an increase of the blockage frequency correlates with a decrease of the residence time.[52,200] Compared to the experiments with PorB, the added antibiotic concentrations are rather high and the obtained residence times are significantly longer. Consequently, when one molecule is released from the binding site, the next is already available and bound immediately. Thus, the residence time limits the maximum blockage frequency. For PorB, the used ampicillin concentration is 5 to 10 -fold lower, reducing the frequency of molecules arriving at the binding site. In addition, the 10 -fold shorter residence times result in a virtually permanently free binding site, so that the frequency of binding - and thus blockage - is not restricted by the residence time. Therefore, the two characteristics of the ampicillin binding are considered separately.

Higher blockage frequencies for the mutant G103K than for the wild type were obtained at $\mathrm{pH} 7.5$ and voltages above $+40 \mathrm{mV}$, as well as at $\mathrm{pH} 6$ and voltages above $+60 \mathrm{mV}$. They indicate a more frequent binding of ampicillin to the mutant than to the wild type, either because of a higher translocation rate, or due to a repeated binding after a release to the same side. 
Molecular dynamics simulations of the transport of enrofloxacin through OmpF revealed a repeated blockage of the pore before translocation. ${ }^{[53]}$ Furthermore, a re-trapping of previously released sugar molecules is reported in case of chitoporin. ${ }^{[191]}$ A possible explanation might be once more a disadvantageous pre-orientation upon approaching the constriction region, caused by the change of the electric field due to the introduction of the positive charge. The ampicillin molecule might have to escape from the binding site and return after adapting a more suitable orientation. ${ }^{[119]}$ Considering the longer residence times in case of the mutant, the orientation after rebinding appears still not to be ideal, hence ampicillin needs to overcome a higher energy barrier. The increased steric hindrance of the lysine residue might play a role in this as well.

Further, different voltage dependencies of the blockage frequencies were found for the two porins - a linear decrease for PorB wt and an increase for PorB G103K. Linearly decreasing frequencies with increasing positive voltage are known for OmpF wt and the zwitterionic form of ampicillin, ${ }^{[52]}$ but no systematic studies exist for charged antibiotics, nor are there investigations on the influence of mutations.

Like already found for the residence times, the general trend of this voltage dependence is not influenced by the charge of ampicillin and can thus only be assigned to PorB itself. Voltage dependent conformational changes of the pore were already discussed to affect the antibiotic's residence time and might as well influence the frequency of interactions between ampicillin and PorB. Due to a changed protein conformation and the resulting alteration of the pore's inner electric field the antibiotic might have to adapt different orientations before entering the constriction zone (pre-orientations).[119] A more or less favorable pre-orientation could not only lead to a reorientation within the constriction zone (see discussion above), but as well force the antibiotic to move out of the constriction zone, reorient and then rebind several times. This pre-orientation is influenced by the pore conformation and therewith by the applied voltage. As the point mutation G103K changes the tendency of the voltage dependence, it seems to affect the pre-orientation of the ampicillin molecule, too, by changing the inner electric field of the pore. As a result, the pre-orientation appears to be more favorable at lower voltages (lower frequency) than at higher ones for the mutant and the other way around in case of the wild type.

Another influence on the pre-orientation appears to be the charge of the ampicillin molecule. Only a slight influence of the applied voltage on the blockage frequency can be observed for PorB wt and the partly anionic ampicillin, while the frequency of the zwitterionic species notably depends on the voltage. Looking at the mutant, the blockage frequency of the partly 
anionic form of ampicillin changes more strongly with the voltage than the one of the zwitterionic species. Furthermore, comparing pH 7.5 and pH 6 for both proteins, higher blockage frequencies of the zwitterionic ampicillin than of the partly anionic species are found. This tendency is more distinct at low voltages and implies a more frequent binding of the zwitterionic antibiotic than of the anionic form. Both effects - the different voltage dependencies for zwitterionic and anionic ampicillin as well as their different blockage frequencies are explainable by the varying dipole between the two species, which influences the orientation of the molecule in the pore.

Steered molecular dynamics (SMD) simulations were performed to further elucidate the permeation of ampicillin through the eyelet, from both, the periplasmic compartment and from the extracellular binding site, especially concerning its orientation (Salomé Llabrés and Ulrich Zachariae, Computational Biology, School of Life Sciences, University of Dundee, UK, unpublished results). While traversing the constriction zone from the extracellular side (inward direction), the antibiotic reorients after leaving the binding site and adopts a vertical orientation, either with the acid or the basic moiety first. In PorB wt, these two possibilities occur with almost the same probability (in 16 out of 30 runs the acid-moiety-first orientation is adopted). By contrast, ampicillin prefers the acid-moiety-first orientation upon crossing the mutant eyelet ( 27 out of 30 runs), which can be attributed to restrictions caused by the additional charge of Lys103. This difference in the preferred orientation of ampicillin between PorB wt and the mutant can explain the opposite voltage dependence found for the blockage frequencies. The applied voltage might alter the inner electric field of the pore, which can support or impede the re-orientation of ampicillin necessary to enter the constriction zone. Different starting orientations influence whether an increasing positive voltage is advantageous or not. As the wild type showed an equal distribution between the two orientations, it is reasonable that the voltage has a weaker influence on the blockage frequency, at least at pH 7.5 (see Figure 4.42).

Further differences were detected by comparing the tilt angle of ampicillin traversing the wild type and the mutant constriction region (see Figure 4.43). Although quite focused near the binding site, this tilt angle is widely spread between $\sim-20^{\circ}$ and $\sim 160^{\circ}$ at the periplasmic exit of the wild types' constriction zone. While crossing the G103K mutant, however, ampicillin exhibits a narrower distribution of the tilt angle, between $\sim-30^{\circ}$ and $\sim 85^{\circ}$ in the second half of the eyelet. In fact, during permeation through the mutant channel, the orientational freedom of the antibiotic appears to be restricted between the extracellular entrance of the eyelet and the periplasmic vestibule. This constraint likely results in an entropic penalty for 
inward permeation of ampicillin through PorB G103K. Thus, the observed higher blockage frequencies for $\mathrm{G} 103 \mathrm{~K}$ would not correspond to a higher permeation rate, but rather be caused by more frequently occurring same-side release of ampicillin due to an unfavorable orientation.
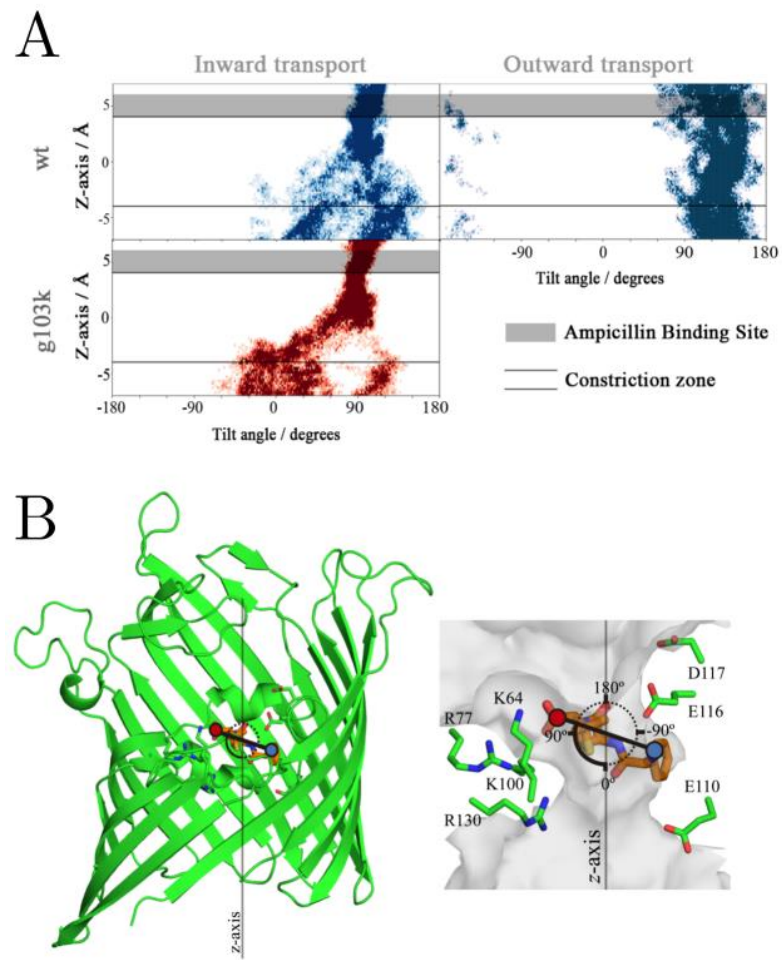

Figure 4.43. Ampicillin tilt angles. Tilt angles upon inward (extracellular towards periplasmic side) and outward (periplasmic towards extracellular) permeation through PorB (A). Definition of the ampicillin tilt angle (B): Angle between the z-axis and the ligand axis formed by the position of the carbon atom of the carboxylic acid and the nitrogen atom of the amino group. (Salomé Llabrés and Ulrich Zachariae, Computational Biology, School of Life Sciences, University of Dundee, UK, unpublished results).

Looking at the permeation of ampicillin from the periplasmic vestibule towards the extracellular side (outward direction), the antibiotic crosses the channel mostly with the acid-moiety facing the extracellular side. Hereby, the tilt angle widely spreads between $\sim 100^{\circ}$ and $165^{\circ}$ within large parts of the eyelet. In contrast to the inward direction, no focusing of the ampicillin orientation was detected, probably due to the absence of a defined binding site at the periplasmic side of the constriction region, which would lead to a pre-orientation of the antibiotic. 
4 Results and Discussion 


\section{Conclusion}

The electrophysiological investigation of PorB wt and the mutants in the absence of ampicillin revealed a reduction of the open channel conductance due to the mutations, more pronounced for G103K than for G103D. Together with the influence of the G->K mutation on the pore's surface potential, this indicates that the nature of the charge is the major cause.

The gating behavior showed a high level of cooperativity between the monomers of PorB wt and PorB G103K, whereas for the G103D mutant a reduction of the cooperativity and more independent gating of the monomers was observed.

The increased open channel noise of PorB G103D compared to PorB wt and PorB G103K is ascribed to pKs shifts of ionizable residues within the pore and electrostatic repulsions between residues at the internal loop.

All three porins prefer the closed state when inserted into solvent-free bilayers while the open state is dominant in black lipid membranes (BLMs). This difference can be rationalized by the organic solvent present in the BLMs, influencing membrane and protein properties.

The electrophysiological measurements in the presence of ampicillin revealed an interaction with the wild type PorB and the mutant G103K, detected by short blockages of the open channel conductance, but no interaction with the mutant G103D was found. A destabilization of the binding site due to electrostatic repulsions between residues at the internal loop might be the reason.

Ampicillin blocks the PorB channel only when added to its extracellular side. Together with the asymmetric location of the binding site at the extracellular side found by molecular dynamics (MD) simulations, this suggests a high energy barrier for ampicillin entering from the periplasmic side. The absence of blockage events at applied negative voltages can be explained by the direction of the electro-osmotic flow (EOF) and its destabilizing effect on the ampicillin binding at negative voltages.

The longer ampicillin residence times obtained for G103K indicate a higher energy barrier for translocation due to an unfavorable orientation upon entering the constriction region, caused by the mutation. The voltage dependence of the residence times is similar for both porins and is thus ascribed to voltage dependent pore conformations. Compared to other porins, the residence times of ampicillin in PorB are 10-fold shorter, implying a relatively 
weak binding between pore and antibiotic. This was reinforced by the huge influence of the EOF on the ampicillin binding stability revealed during MD simulations.

An increased entropic penalty for the permeation of ampicillin through PorB G103K was found in the MD simulations. Consequently, the higher blockage frequencies in comparison to the wild type detected in the electrophysiological experiments can be attributed to an increased rate of same-side release of ampicillin, probably due to an unfavorable orientation. The opposed voltage dependence of the blockage frequencies of wild type and mutant are caused by different orientations while traversing the constriction region.

These results confirm that the mutation G->K at the internal loop of PorB of Neisseria meningitidis indeed influences the interaction between PorB and ampicillin. A reduced permeation of the antibiotic through the bacterial porin due to the mutation is a probable consequence. 


\section{LITERATURE}

[1] B. Alberts, A. Johnson, J. Lewis, M. Raff, K. Roberts, P. Walter, Molecular biology of the cell, 5th edition, Garland Science, New York, 2008.

[2] J. M. Berg, J. L. Tymoczko, L. Stryer, Stryer Biochemie, 6. Aufl., Spektrum Akademischer Verlag, Heidelberg, 2007.

[3] J. B. de la Serna, G. J. Schütz, C. Eggeling, M. Cebecauer, There is no simple model of the plasma membrane organization, Front Cell Dev Biol, 2016, 4, 1-17.

[4] S. J. Singer, G. L. Nicolson, The fluid mosaic model of the structure of cell membranes, Science, 1972, 175, 720-731.

[5] P. G. Saffman, M. Delbrück, Brownian motion in biological membranes, P Natl Acad Sci USA, 1975, 72.

[6] K. Simons, E. Ikonen, Functional rafts in cell membranes, Nature, 1997, 387, 569-572.

[7] O. G. Mouritsen, M. Bloom, Mattress model of lipid-protein interactions in membranes, Biophys J, 1984, 46, 141-153.

[8] D. M. Engelman, Membranes are more mosaic than fluid, Nature, 2005, 438, 578-580.

[9] A. D. Dupuy, D. M. Engelman, Protein area occupancy at the center of the red blood cell membrane, P Natl Acad Sci USA, 2008, 105, 2848-2852.

[10] K. Ritchie, R. Iino, T. Fujiwara, K. Murase, A. Kusumi, The fence and picket structure of the plasma membrane of live cells as revealed by single molecule techniques (Review), Mol Membrane Biol, 2009, 20, 13-18.

[11] D. Lingwood, K. Simons, Lipid rafts as a membrane-organizing principle, Science, 2010, 327, 46-50.

[12] H. Nikaido, Molecular basis of bacterial outer membrane permeability revisited, Microbiol Mol Biol R, 2003, 67, 593-656.

[13] R. Benz, Structure and function of porins from gram-negative bacteria, Annu Rev Microbiol, 1988, 42, 359-393.

[14] H. Nikaido, Porins and specific channels of bacterial outer membranes, Mol Microbiol, 1992, 6, 435-442.

[15] B. K. Ziervogel, B. Roux, The binding of antibiotics in OmpF porin, Structure, 2013, 21, 76-87. 
[16] U. Zachariae, T. Klühspies, S. De, H. Engelhardt, K. Zeth, High resolution crystal structures and molecular dynamics studies reveal substrate binding in the porin Omp32, $J$ Biol Chem, 2006, 281, 7413-7420.

[17] T. Schirmer, General and specific porins from bacterial outer membranes, J Struct Biol, 1998, 121, 101-109.

[18] G. E. Schulz, The structure of bacterial outer membrane proteins, BBA-Biomembranes, 2002, 1565, 308-317.

[19] R. Koebnik, K. P. Locher, P. Van Gelder, Structure and function of bacterial outer membrane proteins: barrels in a nutshell, Mol Microbiol, 2000, 37, 239-253.

[20] A. H. Delcour, Solute uptake through general porins, Front Biosci, 2003, 8, D1055D1071.

[21] RCSB Protein Data Bank, Structures of the OmpF porin crystallized in the presence of foscholine-12, can be found under http://www.rcsb.org/pdb/explore/jmol.do?structureId=3K19\&bionumber=1, 21.06.17.

[22] M. Watanabe, J. Rosenbusch, T. Schirmer, M. Karplus, Computer simulations of the OmpF porin from the outer membrane of Escherichia coli, Biophys J, 1997, 72, 20942102.

[23] P. S. Phale, T. Schirmer, A. Prilipov, K.-L. Lou, A. Hardmeyer, J. P. Rosenbusch, Voltage gating of Escherichia coli porin channels: role of the constriction loop, P Natl Acad Sci $U S A, 1997,94,6741-6745$.

[24] G. Bainbridge, H. Mobasheri, G. A. Armstrong, Lea, E. J. A., J. H. Lakey, Voltage-gating of Escherichia coli porin: a cystine-scanning mutagenesis study of Loop 3, J Mol Biol, 1998, 275, 171-176.

[25] M. A. Arbing, J. W. Hanrahan, J. W. Coulton, Mutagenesis identifies amino acid residues in extracellular loops and within the barrel lumen that determine voltage gating of porin from Haemophilus influenzae type b, Biochemistry, 2001, 40, 14621-14628.

[26] K. M. Robertson, D. P. Tieleman, Molecular basis of voltage gating of OmpF porin, Biochem Cell Biol, 2002, 80, 517-523.

[27] G. Bainbridge, I. Gokce, J. H. Lakey, Voltage gating is a fundamental feature of porin and toxin barrel membrane channels, FEBS Letters, 1998, 431, 305-308.

[28] J. M. Pagès, C. E. James, M. Winterhalter, The porin and the permeating antibiotic: a selective diffusion barrier in gram-negative bacteria, Nat Rev Microbiol, 2008, 6, 893903.

[29] L. B. Rice, Emerging issues in the management of infections caused by multidrug-resistant gram-negative bacteria, Clev Clin J Med, 2007, 74, S12-S20. 
[30] G. Eliopoulos, S. Cosgrove, Y. Carmeli, The impact of antimicrobial resistance on health and economic outcomes, Clin Infect Dis, 2003, 36, 1433-1437.

[31] J. O'Neill, Review on antimicrobial resistance, can be found under https://amr-review.org/sites/default/files/160525_Final\%20paper_with\%20cover.pdf, 13.06.2017.

[32] A. Davin-Regli, J.-M. Bolla, C. James, J.-P. Lavigne, J. Chevalier, E. Garnotel, A. Molitor, J.M. Pagès, Membrane permeability and regulation of drug "influx and efflux" in enterobacterial pathogens, Curr Drug Targets, 2008, 9, 750-759.

[33] G. Wright, Bacterial resistance to antibiotics: enzymatic degradation and modification, Adv Drug Deliver Rev, 2005, 57, 1451-1470.

[34] A. H. Delcour, Outer membrane permeability and antibiotic resistance, BBA-Proteins Proteom, 2009, 1794, 808-816.

[35] M. Tanabe, C. M. Nimigean, T. M. Iverson, Structural basis for solute transport, nucleotide regulation, and immunological recognition of Neisseria meningitidis PorB, P Natl Acad Sci USA, 2010, 107, 6811-6816.

[36] A. Bryskier, Antimicrobial agents, Antibacterials and antifungals, ASM Press, Washington, 2005.

[37] A. Doménech-Sánchez, L. Martínez-Martínez, S. Hernández-Allés, M. del Carmen Conejo, Á. Pascual, J. M. Tomás, S. Albertí, V. J. Benedí, Role of Klebsiella pneumoniae OmpK35 porin in antimicrobial resistance, Antimicrob Agents Ch, 2003, 47, 33323335.

[38] C. S. Toro, S. R. Lobos, I. Calderón, M. Rodríguez, G. C. Mora, Clinical isolate of a porinless Salmonella typhi resistant to high levels of chloramphenicol, Antimicrob Agents Ch, 1990, 34, 1715-1719.

[39] V. Simonet, M. Malléa, J. M. Pagès, Substitutions in the eyelet region disrupt cefepime diffusion through the Escherichia coli OmpF channel, Antimicrob Agents Ch, 2000, 44, 311-315.

[40] A. Thiolas, C. Bornet, A. Davin-Régli, J. M. Pagès, C. Bollet, Resistance to imipenem, cefepime, and cefpirome associated with mutation in Omp36 osmoporin of Enterobacter aerogenes, Biochem Bioph Res Co, 2004, 317, 851-856.

[41] H. Nikaido, E. Y. Rosenberg, Porin channels in Escherichia coli: studies with liposomes reconstituted from purified proteins, J Bacteriol, 1983, 153, 241-252.

[42] M. Virji, Pathogenic neisseriae: surface modulation, pathogenesis and infection control, Nat Rev Micro, 2009, 7, 274-286.

[43] B. A. Oppenheim, Antibiotic resistance in Neisseria meningitidis, Clin Infect Dis, 1997, 24, S98-S101. 
[44] J. W. Tapsall, Antibiotic resistance in Neisseria gonorrhoeae, Clin Infect Dis, 2005, 41, S263-S268.

[45] E. C. Gotschlich, M. E. Seif, M. S. Blake, M. Koomey, Porin protein of Neisseria gonorrhoeae: cloning and gene structure, P Natl Acad Sci USA, 1987, 84, 8135-8139.

[46] K. Murakami, E. C. Gotschlich, M. E. Seif, Cloning and characterization of the structural gene for the class 2 protein of Neisseria meningitidis, Infect Immun, 1989, 57, 23182323.

[47] V. Kozjak-Pavlovic, E. A. Dian-Lothrop, M. Meinecke, O. Kepp, K. Ross, K. Rajalingam, A. Harsman, E. Hauf, V. Brinkmann, D. Günther, I. Herrmann, R. Hurwitz, J. Rassow, R. Wagner, T. Rudel, H. S. Seifert, Bacterial porin disrupts mitochondrial membrane potential and sensitizes host cells to apoptosis, PLoS Pathog, 2009, 5, e1000629_1e1000629_15.

[48] M. Olesky, M. Hobbs, R. A. Nicholas, Identification and analysis of amino acid mutations in Porin IB that mediate intermediate-level resistance to penicillin and tetracycline in Neisseria gonorrhoeae, Antimicrob Agents Ch, 2002, 46, 2811-2820.

[49] M. Olesky, S. Zhao, R. L. Rosenberg, R. A. Nicholas, Porin-mediated antibiotic resistance in Neisseria gonorrhoeae: ion, solute, and antibiotic permeation through PIB Proteins with penB Mutations, J Bacteriol, 2006, 188, 2300-2308.

[50] J. Song, C. A. S. A. Minetti, M. S. Blake, M. Colombini, Successful recovery of the normal electrophysiological properties of PorB (Class 3) porin from Neisseria meningitidis after expression in Escheria coli and renaturation, BBA-Biomembranes, 1998, 1370, 289-298.

[51] L. Kullman, M. Winterhalter, S. M. Bezrukov, Transport of maltodextrins through maltoporin: a single-channel study, Biophys J, 2002, 82, 803-812.

[52] C. Danelon, E. M. Nestorovich, M. Winterhalter, M. Ceccarelli, S. M. Bezrukov, Interaction of zwitterionic penicillins with the $\mathrm{OmpF}$ channel facilitates their translocation, Biophys J, 2006, 90, 1617-1627.

[53] K. R. Mahendran, E. Hajjar, T. Mach, M. Lovelle, A. Kumar, I. Sousa, E. Spiga, H. Weingart, P. Gameiro, M. Winterhalter, M. Ceccarelli, Molecular basis of enrofloxacin translocation through OmpF, an outer membrane channel of Escherichia coli - when binding does not imply translocation, J Phys Chem B, 2010, 114, 5170-5179.

[54] G. Schwarz, C. Danelon, M. Winterhalter, On translocation through a membrane channel via an internal binding site: kinetics and voltage dependence, Biophys J, 2003, 84, 2990-2998. 
[55] C. E. James, K. R. Mahendran, A. Molitor, J. M. Bolla, A. N. Bessonov, M. Winterhalter, J. M. Pagès, D. Fatouros, How $\beta$-Lactam antibiotics enter bacteria: a dialogue with the porins, PLoS One, 2009, 4, e5453.

[56] E. M. Nestorovich, C. Danelon, M. Winterhalter, S. M. Bezrukov, Designed to penetrate: time-resolved interaction of single antibiotic molecules with bacterial pores, $P$ Natl Acad Sci USA, 2002, 99, 9789-9794.

[57] P. Van Gelder, F. Dumas, J. P. Rosenbusch, M. Winterhalter, Oriented channels reveal asymmetric energy barriers for sugar translocation through maltoporin of Escherichia coli, Eur J Biochem, 2000, 267, 79-84.

[58] F. Dumas, R. Koebnik, M. Winterhalter, P. Van Gelder, Sugar transport through maltoporin of Escherichia coli. Role of polar tracks, J Biol Chem, 2000, 275, 19747-19751.

[59] R. Dutzler, T. Schirmer, M. Karplus, S. Fischer, Translocation mechanism of long sugar chains across the maltoporin membrane channel, Structure, 2002, 10, 1273-1284.

[60] A. Kumar, E. Hajjar, P. Ruggerone, M. Ceccarelli, Molecular simulations reveal the mechanism and the determinants for ampicillin translocation through OmpF, J Phys Chem B, 2010, 114, 9608-9616.

[61] W. Im, S. Seefeld, B. Roux, A grand canonical monte carlo-brownian dynamics algorithm for simulating ion channels, Biophys J, 2000, 79, 788-801.

[62] C. Kattner, J. Zaucha, F. Jaenecke, U. Zachariae, M. Tanabe, Identification of a cation transport pathway in Neisseria meningitidis PorB, Proteins, 2013, 81, 830-840.

[63] S. Acosta-Gutierrez, M. A. Scorciapino, I. Bodrenko, M. Ceccarelli, Filtering with electric field: the case of E. coli porins, J Phys Chem Lett, 2015, 6, 1807-1812.

[64] E. K. Schmitt, M. Nurnabi, R. J. Bushby, C. Steinem, Electrically insulating pore-suspending membranes on highly ordered porous alumina obtained from vesicle spreading, Soft Matter, 2008, 4, 250-253.

[65] E. K. Schmitt, C. Weichbrodt, C. Steinem, Impedance analysis of gramicidin D in poresuspending membranes, Soft Matter, 2009, 5, 3347.

[66] I. Mey, M. Stephan, E. K. Schmitt, M. M. Müller, M. Ben Amar, C. Steinem, A. Janshoff, Local membrane mechanics of pore-spanning bilayers, J Am Chem Soc, 2009, 131, 7031-7039.

[67] J. L. Gabriel, P. L. G. Chong, Molecular modeling of archaebacterial bipolar tetraether lipid membranes, Chem Phys Lipids, 2000, 105, 193-200.

[68] R. Nagarajan, Molecular packing parameter and surfactant self-assembly: the neglected role of the surfactant tail, Langmuir, 2002, 18, 31-38. 
[69] H. Lindsey, N. O. Petersen, S. I. Chan, Physicochemical characterization of 1,2-diphytanoyl-sn-glycerol-3-phosphocholine in model membrane systems, $B B A$ Biomembranes, 1979, 555, 147-167.

[70] P. L. Yeagle, Cholesterol and the cell membrane, BBA-Rev Biomembranes, 1985, 822, 267-287.

[71] A. Léonard, C. Escrive, M. Laguerre, E. Pebay-Peyroula, W. Néri, T. Pott., J. Katsaras, E. J. Dufourc, Location of cholesterol in DMPC membranes. A comparative study by neutron diffraction and molecular mechanics simulation, Langmuir, 2001, 17, 20192030.

[72] J. Gallová, D. Uhríková, A. Islamov, A. Kuklin, P. Balgavý, Effect of choleterol on the bilayer thickness in unilamellar extruded DLPC and DOPC liposomes: SANS contrast variation study, Gen Physiol Biophys, 2004, 23, 113-128.

[73] X. Liang, G. Mao, K. Y. S. Ng, Mechanical properties and stability measurement of cholesterol-containing liposome on mica by atomic force microscopy, J Colloid Interf Sci, 2004, 278, 53-62.

[74] C. Kattner, D. N. Toussi, J. Zaucha, L. M. Wetzler, N. Rüppel, U. Zachariae, P. Massari, M. Tanabe, Crystallographic analysis of Neisseria meningitidis PorB extracellular loops potentially implicated in TLR2 recognition, J Struct Biol, 2014, 185, 440-447.

[75] M. Tanabe, T. M. Iverson, Expression, purification and preliminary X-ray analysis of the Neisseria meningitidis outer membrane protein PorB, Acta Crystallogr F, 2009, 65, 996-1000.

[76] RCSB Protein Data Bank, Crystal Structure of outer membrane protein PorB from Neisseria meningitidis, can be found under http://www.rcsb.org/pdb/explore/jmol.do?structureId=3VZT\&bionumber=1, 18.04.2017.

[77] J. P. Hou, J. W. Poole, The amino acid nature of ampicillin and related penicillins, J Pharm Sci, 1969, 58, 1510-1515.

[78] Sirius Analytical Ltd, pKa definitions, can be found under http://www.sirius-analytical.com/science/pka/pka-definitions, 19.04.18.

[79] S. Y. Essack, The development of $\beta$-lactam antibiotics in response to the evolution of $\beta$-lactamamases, Pharm Res, 2001, 18, 1391-1399.

[80] U. K. Laemmli, Cleavage of structural proteins during the assembly of the head of bacteriophage T4, Nature, 1970, 227, 680-685.

[81] D. N. Toussi, M. Carraway, L. M. Wetzler, L. A. Lewis, X. Liu, P. Massari, The amino acid sequence of Neisseria lactamica PorB surface-exposed loops influences Toll-like receptor 2-dependent cell activation, Infect Immun, 2012, 80, 3417-3428. 
[82] C. A. S. A. Minetti, M. S. Blake, D. P. Remeta, Characterization of the structure, function, and conformational stability of PorB class 3 protein from Neisseria meningitidis, J Biol Chem, 1998, 273, 25329-25338.

[83] M. K. Doeven, J. H. A. Folgering, V. Krasnikov, E. R. Geertsma, G. van den Bogaart, B. Poolman, Distribution, lateral mobility and function of membrane proteins incorporated into giant unilamellar vesicles, Biophys J, 2005, 88, 1134-1142.

[84] N. F. Morales-Penningston, J. Wu, E. R. Farkas, S. L. Goh, T. M. Konyakhina, J. Y. Zheng, W. W. Webb, G. W. Feigenson, GUV preparation and imaging: minimizing artifacts, BBA-Biomembranes, 2010, 1798, 1324-1332.

[85] R. Dimova, K. A. Riske, S. Aranda, N. Bezlyepkina, R. L. Knorr, R. Lipowsky, Giant vesicles in electric fields, Soft Matter, 2007, 3, 817.

[86] K. S. Cole, Dynamic electrical characteristics of the squid axon membrane, Arch Sci Physiol, 1949, 3, 253-258.

[87] G. Marmont, Studies on the axon membrane. I. A new method, J Cell Physiol, 1949, 34, 351-382.

[88] E. Neher, B. Sakman, Single-channel currents recorded from membrane denervated frog muscle fibres, Nature, 1976, 260, 799-802.

[89] E. Neher, Ion channels for communication between and within cells, Bioscience Rep, $1992,12$.

[90] R. Horn, J. Patlak, Single channel currents from excised patches of muscle membrane, P Natl Acad Sci USA, 1980, 77, 6930-6934.

[91] O. Hamill, A. Marty, E. Neher, B. Sakmann, F. J. Sigworth, Improved patch-clamp techniques for high-resolution current recording from cells and cell-free membrane patches, Pflügers Arch, 1981, 391, 85-100.

[92] Molecular Devices, The axon guide: a guide to electrophysiology \& biophysics laboratory techniques, 2008.

[93] R. M. Hochmuth, C. A. Evans, H. C. Wiles, J. T. McCown, Mechanical measurement of red cell membrane thickness, Science, 1983, 220, 101-102.

[94] R. Hine, The facts on file dictionary of biology, 4th edition, Checkmark Books/Facts On File, New York, 2005.

[95] L. J. Gentet, G. J. Stuart, J. D. Clements, Direct measurement of specific membrane capacitance in neurons, Biophys J, 2000, 79, 314-320.

[96] W. Liang, Y. Zhao, L. Liu, Y. Wang, W. J. Li, G.-B. Lee, Determination of cell membrane capacitance and conductance via optically induced electrokinetics, Biophys J, 2017, 113, 1531-1539. 
[97] Axon Instruments Inc., Axopatch 200B: patch-clamp theory and operation, 19971999.

[98] Nanion Technologies GmbH, Lipid bilayer recordings of OmpF reconstituted in proteoliposomes, can be found under http://www.nanion.de/images/stories/pdf/OmpfPortaPatch.pdf, 04.07.2017.

[99] Nanion Technologies GmbH, Lipid bilayer recordings of KcsA channels reconstituted in proteliposomes, can be found under http://www.nanion.de/images/stories/pdf/Port-a-Patch_BilayerKcsA.pdf, 04.07.2017.

[100] A. Helenius, K. Simons, Solubilization of membranes by detergents, BBA-Rev Biomembranes, 1975, 415, 29-79.

[101] C. Danelon, T. Brando, M. Winterhalter, Probing the orientation of reconstituted maltoporin channels at the single-protein level, J Biol Chem, 2003, 278, 35542-35551.

[102] T. L. Jones, R. Fu, F. Nielson, T. A. Cross, D. D. Busath, Gramicidin channels are internally gated, Biophys J, 2010, 98, 1486-1493.

[103] E. M. Nestorovich, T. K. Rostovtseva, S. M. Bezrukov, Residue ionization and ion transport through OmpF channels, Biophys J, 2003, 85, 3718-3729.

[104] M. Winterhalter, Black lipid membranes, Curr Opin Colloid Interface Sci, 2000, 5, 250255.

[105] A. Johannsson, C. A. Keightley, G. A. Smith, C. D. Richards, T. R. Hesketh, J. C. Metcalfe, The effect of bilayer thickness and n-alkanes on the activity of the $\left(\mathrm{Ca}^{2+}+\mathrm{Mg}^{2+}\right)$-dependent ATPase of sacroplasmic reticulum, J Biol Chem, 1981, 256, 1643-1650.

[106] R. B. Gregory, Protein-solvent interactions, Marcel Dekker Inc., New York, 1995.

[107] Nanion Technologies GmbH, The world's smallest patch-clamp setup. Providing biggest results., can be found under http://www.nanion.de/en/application-database/database-sorted-by-instruments/category/13-product-sheets-flyer.html, 30.12.2017.

[108] Nanion Technologies GmbH, Orbit 16. Instant bilayers. Just add protein., can be found under file:///C:/Users/Annika/Downloads/Nanion_Product_Sheet_Orbit16.pdf, 06.01.2018.

[109] D. Freedman, P. Diaconis, On the histogram as a density estimator: $L_{2}$ theory, Probab Theory Rel, 1981, 57, 453-476.

[110] F. Pein, I. Tecuapetla-Gómez, O. M. Schütte, C. Steinem, A. Munk, Fully-automatic multiresolution idealization for filtered ion channel recordings: flickering event detection, can be found under https://arxiv.org/abs/1706.03671, 05.01.18.

[111] R. Fletcher, Practical methods of optimization, 2nd edition, John Wiley and Sons, New York, 1987. 
[112] B. J. Alder, T. E. Wainwright, Phase transition for a hard sphere system, J Chem Phys, 1957, 27, 1208-1209.

[113] B. J. Alder, T. E. Wainwright, Studies in molecular dynamics. I. General method, J Chem Phys, 1959, 31, 459-466.

[114] J. A. McCammon, B. R. Gelin, M. Karplus, Dynamics of folded proteins, Nature, 1977, $267,585-590$.

[115] M. Karplus, Molecular dynamics simulations of biomolecules, Acc Chem Res, 2002, 35, 321-323.

[116] C. Kandt, W. L. Ash, D. P. Tieleman, Setting up and running molecular dynamics simulations of membrane proteins, Methods, 2007, 41, 475-488.

[117] A. R. Leach, Molecular modelling: principles and applications, 2nd edition, Pearson Education Limited, Harlow, 2001.

[118] M. Ceccarelli, C. Danelon, A. Laio, M. Parrinello, Microscopic mechanism of antibiotics translocation through a porin, Biophys J, 2004, 87, 58-64.

[119] H. Bajaj, M. A. Scorciapino, L. Moynié, M. G. P. Page, J. H. Naismith, M. Ceccarelli, M. Winterhalter, Molecular basis of filtering carbapenems by porins from $\beta$-lactam-resistant clinical strains of Escherichia coli, J Biol Chem, 2016, 291, 2837-2847.

[120] B. Isralewitz, M. Gao, K. Schulten, Steered molecular dynamics and mechanical functions of proteins, Curr Opin Struc Biol, 2001, 11, 224-230.

[121] S. O. Yesylevskyy, ProtSqueeze: simple and effective automated tool for setting up membrane protein simulations, J Chem Inf Model, 2007, 47, 1986-1994.

[122] M. G. Wolf, M. Hoefling, C. Aponte-Santamaría, H. Grubmüller, G. Groenhof, g_membed: efficient insertion of a membrane protein into an equilibrated lipid bilayer with minimal perturbation, J Comput Chem, 2010, 31, 2169-2174.

[123] K. A. Feenstra, B. Hess, H. J. C. Berendsen, Improving efficiency of large time-scale molecular dynamics simulations of hydrogen-rich systems, J Comput Chem, 1999, 20, 786-798.

[124] K. Lindorff-Larsen, S. Piana, K. Palmo, P. Maragakis, J. L. Klepeis, R. O. Dror, D. E. Shaw, Improved side-chain torsion potentials for the Amber ff99SB protein force field, Proteins, 2010, 78, 1950-1958.

[125] A. Cordomí, G. Caltabiano, L. Pardo, Membrane protein simulations using AMBER force field and Berger lipid parameters, J Chem Theory Comput, 2012, 8, 948-958.

[126] E. Lindahl, O. Edholm, Mesoscopic undulations and thickness fluctuations in lipid bilayers from molecular dynamics simulations, Biophys J, 2000, 79, 426-433. 
[127] I. S. Joung, T. E. Cheatham III, Determination of alkali and halide monovalent ion pa-

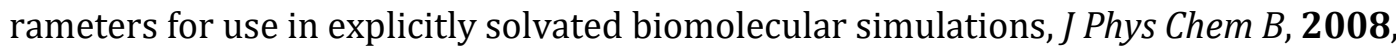
112, 9020-9041.

[128] J. Wang, R. M. Wolf, J. W. Caldwell, P. A. Kollman, D. A. Case, Development and testing of a general amber force field, J Comput Chem, 2004, 25, 1157-1174.

[129] J. Wang, P. Cieplak, P. A. Kollman, How well does a restrained electrostatic potential (RESP) model perform in calculating conformational energies of organic and biological molecules?, J Comput Chem, 2000, 21, 1049-1074.

[130] D. A. Case, T. A. Darden, T. E. Cheatham III, C. L. I. Simmerling, J. Wang, R. E. Duke, B. Roberts, University of California, 2012.

[131] G. Jones, P. Willett, R. C. Glen, A. R. Leach, R. Taylor, Development and validation of a genetic algorithm for flexible docking, J Mol Biol, 1997, 267, 727-748.

[132] S. D. Morley, M. Afshar, Validation of an empirical RNA-ligand scoring function for fast flexible docking using RiboDock®, J Comput Aid Mol Des, 2004, 18, 189-208.

[133] X. Barril, R. E. Hubbrad, S. D. Moorley, Virtual screening in structure-based drug discovery, Mini Rev Med Chem, 2004, 4, 779-791.

[134] M. J. Abraham, T. Murtola, R. Schulz, S. Páll, J. C. Smith, B. Hess, E. Lindahl, GROMACS: high performance molecular simulations through multi-level parallelism from laptops to supercomputers, SoftwareX, 2015, 1-2, 19-25.

[135] G. Bussi, D. Donadio, M. Parrinello, Canonical sampling through velocity rescaling, J Chem Phys, 2007, 126, 14101.

[136] H. J. C. Berendsen, J. P. M. Postma, W. F. van Gunsteren, A. DiNola, J. R. Haak, Molecular dynamics with coupling to an external bath, J Chem Phys, 1984, 81, 3684-3690.

[137] T. Darden, D. York, L. Pedersen, Particle mesh Ewald: an $N \cdot \log (N)$ method for Ewald sums in large systems, J Chem Phys, 1993, 98, 10089-10092.

[138] B. Hess, H. Bekker, H. J. C. Berendsen, J. Fraaije, LINCS: a linear constraint solver for comparative protein modeling, J Comput Chem, 1997, 18, 1463-1472.

[139] S. Miyamoto, P. A. Kollman, Settle: an analytical version of the SHAKE and RATTLE algorithm for rigid water models, J Comput Chem, 1992, 13, 952-962.

[140] C. Kutzner, H. Grubmüller, B. L. de Groot, U. Zachariae, Computational electrophysiology: the molecular dynamics of ion channel permeation and selectivity in atomistic detail, Biophys J, 2011, 101, 809-817.

[141] N. Michaud-Agrawal, E. J. Denning, T. B. Woolf, O. Beckstein, MDAnalysis: a toolkit for the analysis of molecular dynamics simulations, J Comput Chem, 2011, 32, 2319-2327. 
[142] R. T. McGibbon, K. A. Beauchamp, M. P. Harrigan, C. Klein, J. M. Swails, C. X. Hernández, C. R. Schwantes, L.-P. Wang, T. J. Lane, V. S. Pande, MDTraj: a modern open library for the analysis of molecular dynamics trajectories, Biophys $J, 2015,109,1528-1532$.

[143] H. Van Zutphen, A. J. Merole, G. P. Brierley, D. G. Cornwell, The interaction of nonionic detergents with lipid bilayer membranes, Arch Biochem Biophys, 1972, 152, 755-766.

[144] W. D. Seufert, The interaction of anionic detergents with lipid bilayer membranes, Biophysik, 1973, 10, 281-292.

[145] M. Dubinčkova, M. Kiseley, S. Kutuzov, F. Devínski, V. Gordeliy, P. Balgavý, Effect of Nlauryl-N,N-dimethylamine $\mathrm{N}$-oxide on dimyristoyl phosphatidylcholine bilayer thickness: a small-angle neutron scattering study, Gen Physiol Biophys, 1997, 16, 175-188.

[146] T. Rudel, A. Schmid, R. Benz, H. A. Kolb, F. Lang, T. F. Meyer, Modulation of Neisseria porin (PorB) by cytosolic ATP/GTP of target cells: parallels between pathogen accommodation and mitochondrial endosymbiosis, Cell, 1996, 85, 391-402.

[147] D. Jeanteur, T. Schirmer, D. Fourel, V. Simonet, G. Rummel, C. Widmer, J. P. Rosenbusch, F. Pattus, J. M. Pagès, Structural and functional alterations of a colicin-resistant mutant of OmpF porin from Escherichia coli, P Natl Acad Sci USA, 1994, 91, 1067510679.

[148] E. Dé, A. Baslé, M. Jaquinod, N. Saint, M. Malléa, G. Molle, J. M. Pagès, A new mechanism of antibiotic resistance in Enterobacteriaceae induced by a structural modification of the major porin, Mol Microbiol, 2001, 41, 189-198.

[149] C. Kattner, Dissertation, Martin-Luther-Universität, Halle-Wittenberg, 2015.

[150] G. Xu, B. Shi, E. J. McGroarty, H. T. Tien, Channel-closing activity of porins from Escherichia coli in bilayer lipid membranes, BBA-Biomembranes, 1986, 862, 57-64.

[151] J. C. Todt, W. J. Rocque, E. J. McGroarty, Effects of pH on bacterial porin function, Biochemistry-US, 1992, 31, 10471-10478.

[152] P. S. Phale, A. Philippsen, T. Kiefhaber, R. Koebnik, V. P. Phale, T. Schirmer, J. P. Rosenbusch, Stability of trimeric OmpF porin: the contributions of the latching loop L2, Biochemistry, 1998, 37, 15663-15670.

[153] B. Lauman, M. Pagel, A. H. Delcour, Altered pore properties and kinetic changes in mutants of the Vibrio cholerae Porin OmpU, Mol Membrane Biol, 2009, 25, 498-505.

[154] N. Liu, A. H. Delcour, The spontaneous gating activity of OmpC porin is affected by mutations of a putative hydrogen bond network or of a salt bridge between the L3 loop and the barrel, Protein Eng, 1998, 11, 797-802. 
[155] N. Liu, H. Samartzidou, K. W. Lee, J. M. Briggs, A. H. Delcour, 2000. Effects of pore mutations and permeant ion concentration on the spontaneous gating activity of $\mathrm{OmpC}$ porin, Protein Eng, 2000, 13, 491-500.

[156] A. Bartsch, S. Llabrés, F. Pein, C. Kattner, M. Schön, M. Diehn, M. Tanabe, A. Munk, U. Zachariae, C. Steinem, High-resolution experimental and computational electrophysiology reveals weak $\beta$-lactam binding events in the porin PorB, can be found under https://www.biorxiv.org/content/early/2018/04/18/303891, 26.05.18.

[157] J. D. E. Young, M. Blake, A. Mauro, Z. A. Cohn, Properties of the major outer membrane protein from Neisseria gonorrhoeae incorporated into model lipid membranes, $P$ Natl Acad Sci USA, 1983, 80, 3831-3835.

[158] S. R. Jadhav, K. S. Rao, Y. Zheng, R. M. Garavito, R. M. Worden, Voltage dependent closure of PorB class II porin from Neisseria meningitidis investigated using impedance spectroscopy in a tethered bilayer lipid membrane interface, J Colloid Interf Sci, 2013, 390, 211-216.

[159] H. Samartzidou, A. H. Delcour, E.coli PhoE porin has an opposite voltage-dependence to the homologous OmpF, EMBO J, 1998, 17, 93-100.

[160] D. B. Tikhonov, L. G. Magazanik, Voltage dependence of open channel blockade: onset and offset rates, J Membrane Biol, 1998, 161, 1-8.

[161] O. V. Krasilnikov, P. G. Merzlyak, L. N. Yuldasheva, M. F. Capistrano, Protein electrostriction: a possibility of elastic deformation of the $\alpha$-hemolysin channel by the applied field, Eur Biophys J, 2005, 34, 997-1006.

[162] A. Aksimentiev, K. Schulten, Imaging $\alpha$-hemolysin with molecular dynamics: ionic conductance, osmotic permeability, and the electrostatic potential map, Biophys J, 2005, $88,3745-3761$.

[163] N. Saint, K.-L. Lou, C. Widmer, M. Luckey, T. Schirmer, J. P. Rosenbusch, Structural and functional characterization of OmpF porin mutants selected for larger pore size, J Biol Chem, 1996, 271, 20676-20680.

[164] H. Lou, M. Chen, S. S. Black, S. R. Bushell, M. Ceccarelli, T. Mach, K. Beis, A. S. Low, V. A. Bamford, I. R. Booth, H. Bayley, J. H. Naismith, H. W. van Veen, Altered antibiotic transport in OmpC mutants isolated from a series of clinical strains of multi-drug resistant E. coli, PLoS One, 2011, 6, e25825.

[165] H. Miedema, M. Vrouenraets, J. Wierenga, B. Eisenberg, T. Schirmer, A. Baslé, W. Meijberg, Conductance and selectivity fluctuations in D127 mutants of the bacterial porin OmpF, Eur Biophys J, 2006, 36, 13-22. 
[166] A. Baslé, R. Iyer, A. H. Delcour, Subconductance states in OmpF gating, $B B A$ Biomembranes, 2004, 1664, 100-107.

[167] C. Berrier, A. Coulombe, C. Houssin, A. Ghazi, Fast and slow kinetics of porin channels from Escherichia coli reconstituted into giant liposomes and studied by patch-clamp, FEBS Letters, 1992, 306, 251-256.

[168] N. Doukyu, H. Ogino, Organic solvent-tolerant enzymes, Biochem Eng J, 2010, 48, 270282.

[169] D. A. Cowan, Thermophilic proteins: stability and function in aqueous and organic solvents, Comp Biochem Physiol, 1997, 118A, 429-438.

[170] A. M. Klibanov, Improving enzymes by using them in organic solvents, Nature, 2001, 409, 241-246.

[171] C. Weichbrodt, H. Bajaj, G. Baaken, J. Wang, S. Guinot, M. Kreir, J. C. Behrends, M. Winterhalter, N. Fertig, Antibiotic translocation through porins studied in planar lipid bilayers using parallel platforms, Analyst, 2015, 140, 4874-4881.

[172] R. Fettiplace, D. M. Andrews, D. A. Haydon, The thickness, composition and structure of some lipid bilayers and natural membranes, J Membrane Biol, 1971, 5, 277-296.

[173] H. Sackin, Mechanosensitive channels, Annu Rev Physiol, 1995, 57, 333-353.

[174] E. Neher, H. Eibl, The influence of phospholpid polar groups on gramicidin channels, BBA-Biomembranes, 1977, 464, 37-44.

[175] S. B. Hladky, D. A. Haydon, Ion transfer across lipid membranes in the presence of Gramicidin A I. Studies of the unit conductance channel, BBA-Biomembranes, 1972, 274, 294-312.

[176] H.-D. Arndt, A. Knoll, U. Koert, Synthesis of minigramicidin ion channels and test of their hydrophobic match with the membrane, ChemBioChem, 2001, 3, 221-223.

[177] J. A. Lundbaek, S. A. Collingwood, H. I. Ingolfsson, R. Kapoor, O. S. Andersen, Lipid bilayer regulation of membrane protein function: gramicidin channels as molecular force probes, J R Soc Interface, 2010, 7, 373-395.

[178] A. Carruthers, D. L. Melchior, Human erythrocyte hexose transporter activity is governed by bilayer lipid composition in reconstituted vesicles, Biochemistry, 1984, 23, 6901-6911.

[179] G. In 't Veld, A. J. M. Driessen, J. A. F. Op den Kamp, W. N. Konings, Hydrophobic membrane thickness and lipid-protein interactions of the leucine transport system of Lactococcus lactis, BBA-Biomembranes, 1991, 1065, 203-212. 
[180] Y. Uratani, N. Wakayama, T. Hoshino, Effect of lipid acyl chain length on activity of sodium-dependent leucine transport system in Pseudomonas aeruginosa, J Biol Chem, 1987, 262, 16914-16919.

[181] C. Montecucco, G. A. Smith, F. Dabbeni-sala, A. Johannsson, Y. M. Galante, R. Bisson, Bilayer thickness and enzymatic activity in the mitochondrial cytochrome $c$ oxidase and ATPase complex, FEBS Letters, 1982, 144, 145-148.

[182] Ö. Yildiz, K. R. Vinothukmar, P. Goswami, W. Kühlbrandt, Structure of the monomeric outer-membrane porin OmpG in the open and closed conformation, EMBO J, 2006, 25 , 3702-3713.

[183] S. M. Bezrukov, M. Winterhalter, Examining noise sources at the single-molecule level: 1/f noise of an open maltoporin channel, Phys Rev Lett, 2000, 85, 202-205.

[184] F. J. Sigworth, Open channel noise. I. Noise in acetylcholine receptor currents suggests conformational fluctuations, Biophys J, 47, 709-720.

[185] I. G. Abidor, V. B. Arakelyan, L. V. Chernomordik, Y. A. Chizmadzhev, V. F. Pastushenko, M. R. Tarasevich, Electric breakdown of bilayer lipid membranes I. The main experimental facts and their qualitative discussion, J Electroanal Chem, 1979, 104, 37-52.

[186] M. B. Partenskii, V. L. Dorman, P. C. Jordan, Membrane stability under electrical stress: a nonlocal electroelastic treatment, J Chem Phys, 1998, 109, 10361-10371.

[187] P. Sens, H. Isambert, Undulation instability of lipid membranes under an electric field, Phys Rev Lett, 2002, 88, 128102_1-128102_4.

[188] C. Chen, S. W. Smye, M. P. Robinson, J. A. Evans, Membrane electroporation theories: a review, Med Bio Eng Comput, 2006, 44, 5-14.

[189] J. M. Crowley, Electrical breakdown of bimolecular lipid membranes as an electromechanical instability, Biophys J, 1973, 13, 711-724.

[190] S. M. Bezrukov, L. Kullman, M. Winterhalter, Probing sugar translocation through maltoporin at the single channel level, FEBS Letters, 2000, 476, 224-228.

[191] W. Suginta, M. Winterhalter, M. F. Smith, Correlated trapping of sugar molecules by the trimeric protein channel chitoporin, BBA-Biomembranes, 2016, 1858, 3032-3040.

[192] M. Brunen, H. Engelhardt, Asymmetry of orientation and voltage gating of the Acidovorax delafieldii porin Omp34 in lipid bilayers, Eur J Biochem, 1993, 212, 129-135.

[193] C. Danelon, A. Suenaga, M. Winterhalter, I. Yamato, Molecular origin of the cation selectivity in OmpF porin: single channel conductances vs. free energy calculation, Biophys Chem, 2003, 104, 591-603.

[194] G. Vasanthi, S. Krishnaswamy, Dipole moment analysis of membrane proteins suggests role in orientation in the membrane, Indian J Biochem Bio, 2002, 39, 93-100. 
[195] G. Vasanthi, S. Krishnaswamy, Dipole moment in TIM $\alpha / \beta$ fold proteins, Indian J Biochem Bio, 2003, 40, 194-202.

[196] C. Andersen, B. Schiffler, A. Charbit, R. Benz, pH-induced collapse of the extracellular loops closes Escherichia coli maltoporin and allows the study of asymmetric sugar binding, J Biol Chem, 2002, 277, 41318-41325.

[197] T. Schirmer, T. A. Keller, Y. F. Wang, J. P. Rosenbusch, Structural basis for sugar translocation through maltoporin channels at 3.1 Å resolution, Science, 1995, 267, 512514.

[198] S. P. Bhamidimarri, J. D. Prajapati, B. van den Berg, M. Winterhalter, J. L. Klepeis, Role of electroosmosis in the permeation of neutral molecules: CymA and Cyclodextrin as an example, Biophys J, 2016, 110, 600-611.

[199] P. R. Singh, M. Ceccarelli, M. Lovelle, M. Winterhalter, K. R. Mahendran, Antibiotic permeation across the $\mathrm{OmpF}$ channel: modulation of the affinity site in the presence of magnesium, J Phys Chem B, 2012, 116, 4433-4438.

[200] E. Hajjar, K. R. Mahendran, A. Kumar, A. Bessonov, M. Petrescu, H. Weingart, P. Ruggerone, M. Winterhalter, M. Ceccarelli, Bridging timescales and length scales: from macroscopic flux to the molecular mechanism of antibiotic diffusion through porins, Biophys J, 2010, 98, 569-575. 
6. Literature 


\section{APPENDIX}

\subsection{List of figures}

Figure 1.1 Lateral organization of the plasma membrane. …....................................................... 3

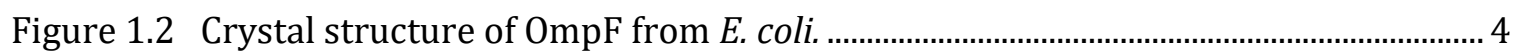

Figure 1.3 Alterations of the antibiotic's permeation related to porin modifications. ............ 6

Figure 1.4 Proposed mechanism for the role of PorB (Ngo) during disease progression. ..... 7

Figure 1.5 Trimeric structure of PorB (Nme). ……….................................................................. 8

Figure 1.6 Binding sites and putative translocation pathways through PorB (Nme). ............. 9

Figure 1.7 Model and schematic conductivity traces of a porin trimer. .......................................10

Figure 3.1 Structure of 1,2-Diphytanoyl-sn-glycero-3-phosphocholin (DPhPC). ....................17

Figure 3.2 Structure of Cholesterol (Chol). ...................................................................................18

Figure 3.3 Amino acid sequence of PorB wild type. ........................................................................18

Figure 3.4 Structure of the $\beta$-lactam antibiotic ampicillin. ..........................................................19

Figure 3.5 Electrical properties of a lipid membrane. …………………………............................23

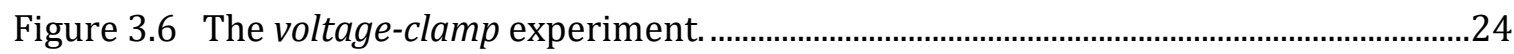

Figure 3.7 Electronic setup of a patch-clamp experiment. ..........................................................2

Figure 3.8 Preparation of a solvent-free membrane. …………….................................................26

Figure 3.9 Preparation of a black lipid membrane (BLM). ……...................................................2

Figure 3.10 Borosilicate substrate used for the measurements at the Port-a-Patch. ...............28

Figure 3.11 Patch-clamp experiment using the Port-a-Patch. .........................................................28

Figure 3.12 Patch-clamp experiment using the self-constructed BLM setup. ............................30

Figure 3.13 Preparation of the BLM measuring chamber. ...............................................................31

Figure 3.14 Aperture in the PTFE foil visualized using the transmitted light microscope. ...32 
Figure 3.15 Patch-clamp experiment using the Orbit 16.

Figure 3.16 Analysis of a conductivity trace showing cannel gating. 34

Figure 3.17 Noise analysis using the MATLAB script PorB.m. 37

Figure 3.18 The analysis routine JULES.

Figure 4.1 No-SDS gel of PorB wt, PorB G103D and PorB G103K.

Figure 4.2 Conductivity trace of a blank sample recorded at $+40 \mathrm{mV}$ and corresponding point amplitude histogram. 46

Figure 4.3 Conductivity event histogram of the blank samples with Gaussian fit. 47

Figure 4.4 Conductivity trace and corresponding point amplitude histogram of PorB wt in a solvent-free membrane at an applied voltage of $+40 \mathrm{mV}$.

Figure 4.5 Conductivity event histogram of PorB wt in solvent-free membranes (5269 events) and Gaussian fit (red line).

Figure 4.6 Point amplitude histograms and Gaussian fits (red lines) of the PorB wt monomer (A), dimer (B) and trimer (C).

Figure 4.7 Conductivity trace of PorB G103K in solvent-free membranes recorded at $+40 \mathrm{mV}$ and corresponding point amplitude histogram.

Figure 4.8 Conductivity event histogram of PorB G103K in solvent-free membranes (3461 events) and Gaussian fit (red line).

Figure 4.9 Point amplitude histograms and Gaussian fits (red lines) of the PorB G103K monomer (A), dimer (B) and trimer (C).

Figure 4.10 Conductivity trace of PorB G103D recorded at $+40 \mathrm{mV}$ in solvent-free membranes.

Figure 4.11 Unusual conductivity trace of PorB G103D recorded in solvent-free membranes at $+40 \mathrm{mV}$.

Figure 4.12 Conductivity event histogram of PorB G103D in solvent-free membranes (11094 events) and Gaussian fit (red line).

Figure 4.13 Point amplitude histogram of the conductivity trace showing frequent gating of PorB G103D. 55

Figure 4.14 Amplitude histograms and Gaussian fits (red lines) of the PorB G103D monomer $(\mathrm{A})$, dimer (B) and trimer (C). 55 
Figure 4.15 Electrostatic surface potential of PorB wt (A, C) and G103K (B, D). .58

Figure 4.16 Trimeric structure of PorB wt (Nme) with different coloring of the monomers.60

Figure 4.17 Section of a conductivity trace of PorB wt in a BLM recorded at $+40 \mathrm{mV}$ and corresponding point amplitude histogram. .63

Figure 4.18 Conductivity event histogram of PorB wt recorded in BLMs at $+40 \mathrm{mV}$ and Gaussian fit (red line). 64

Figure 4.19 Voltage dependent conductivities and open probabilities of PorB wt in BLMs. .65

Figure 4.20 Exemplary conductivity trace with very frequent gating of PorB G103K recorded in BLMs at $+40 \mathrm{mV}$ and corresponding point amplitude histogram. .66

Figure 4.21 Conductivity event histogram of PorB G103K (4780 events) in BLMs at $+40 \mathrm{mV}$ and Gaussian fit (red line). .66

Figure 4.22 Voltage dependent conductivities and open probabilities of PorB G103K in BLMs.

Figure 4.23 Conductivity trace with frequent gating of PorB G103D recorded in BLMs at $+40 \mathrm{mV}$. 68

Figure 4.24 Conductivity event histogram of PorB G103D recorded in BLMs at $+40 \mathrm{mV}$ and Gaussian fit (red line).

Figure 4.25 Voltage dependent conductivities and open probabilities of the PorB G103D trimer in BLMs. .70

Figure 4.26 Open-channel-noise of PorB wt (A), G103K (B) and G103D (C). .............................70

Figure 4.27 Noise frequencies of PorB wt (blue), G103K (red) and G103D (green). ...............71

Figure 4.28 Crystal structure of a PorB (Nme) wt monomer. . .79

Figure 4.29 Section of a conductivity trace of PorB G103D inserted into a BLM, recorded at $+40 \mathrm{mV}$. .80

Figure 4.30 . Exemplary conductivity traces of PorB wt (A and B), G103K (C and D) and G103D (E and F) in the absence (A, C, E) and presence of $1 \mathrm{mM}$ ampicillin $(\mathrm{B}, \mathrm{D}, \mathrm{F}) . . . .86$

Figure 4.31 Exemplary power spectral densities of PorB G103K in the absence (blue) and presence of $1 \mathrm{mM}$ ampicillin. .87

Figure 4.32 Evaluation of the experiments in presence of ampicillin. .89 
Figure 4.33 Amplitude event histograms of PorB wt (A and B), G103K (C and D) and G103D (E and F) in the absence (A, C, E) and presence of $1 \mathrm{mM}$ ampicillin (B, D, E) .... 90

Figure 4.34 Voltage dependent amplitude event histograms of PorB wt (A and B) and G103K (C and D) in absence (A and C) and presence of $1 \mathrm{mM}$ ampicillin (B and D)......91 Figure 4.35 Amplitudes blocked by ampicillin $(1 \mathrm{mM})$ at increasing voltages for PorB wt (blue) and G103K (red).

Figure 4.36 Amplitude event histograms of PorB wt (A) and G103K (B) for increasing ampicillin concentrations.

Figure 4.37 Location of the ampicillin binding site. . .97

Figure 4.38 Direction of the electro-osmotic flow (EOF) and its effect on the ampicillin binding mode at positive (A) and negative (B) voltages. 100

Figure 4.39 Exemplary residence times of ampicillin with confidence intervals (bars) for PorB wt (blue) and G103K (red) at increasing ampicillin concentrations. 101

Figure 4.40 Voltage dependent residence times of ampicillin (1 mM) for PorB wt (blue) and G103K (red) with "guide-to-the-eye" (lines). 103

Figure 4.41 Exemplary concentration dependent blockage frequencies for PorB wt (blue) and G103K (red) with confidence intervals (bars). 105

Figure 4.42 Voltage dependent blockage frequencies for PorB wild type (blue) and the mutant G103K (red) in the presence of $1 \mathrm{mM}$ ampicillin. 106

Figure 4.43 Ampicillin tilt angles. 109 


\subsection{List of tables}

Table 3.1 Compositions of solutions and buffers used for the gel electrophoresis. .20

Table 4.1 Conductivities of PorB wt, G103K and G103D in solvent-free membranes. .56

Table 4.2 Open probabilities of PorB wt, G103K and G103D in solvent-free membranes. ....61

Table 4.3 Conductivities of PorB wt, G103K and G103D in BLMs recorded at $+40 \mathrm{mV}$. .........72

Table 4.4 Trimer open probabilities of PorB wt, G103K and G103D recorded at +40 and $+100 \mathrm{mV}$ in BLMs. .76

Table 4.5 Monomer, dimer and trimer conductivities of PorB wt, G103K and G103D recorded at $+40 \mathrm{mV}$ in solvent-free bilayers and BLMs. . .81

Table 4.6 Open probabilities of PorB wt, G103K and G103D recorded at $+40 \mathrm{mV}$ in solventfree membranes and BLMs.

\subsection{Symbols and abbreviations}

$\begin{array}{ll}\text { A } & \text { alanine } \\ \text { abf } & \text { area, amplitude } \\ \text { A/D } & \text { Adobe binary screen font } \\ \text { analog/digital } \\ \text { AMP-PNP } & \text { adenylylimidodiphosphate } \\ \text { APS } & \text { ammonium peroxosulfate } \\ \text { Arg } & \text { arginine } \\ \text { Asp } & \text { aspartic acid } \\ \text { ATP } & \text { adenosine triphosphate } \\ \text { BLM } & \text { black lipid membrane } \\ \text { C } & \text { capacitance } \\ \text { Chol } & \text { cholesterol } \\ \text { CI } & \text { confidence interval } \\ C_{\mathrm{m}} & \text { membrane capacitance } \\ \text { CR } & \text { constriction region }\end{array}$


d

D

$\delta$

DPhPC

DTT

E

$\mathcal{E}$

$\mathcal{E}_{0}$

EC

E. Coli

EOF

$f$

$f_{\mathrm{c}}$

FWHM

G

G

$G_{\mathrm{c}}$

Glu

Gly

$G_{\mathrm{m}}$

$G_{\mathrm{m}, \mathrm{c}}$

GUV

HEPES

I

IMM

IQR

ITO

JULES

$\mathrm{K}$

$k_{\text {off }}$

$k_{\text {on }}$

LamB

LDAO

Lys diameter, distance

aspartic acid, dimer

interval width

1,2-diphytanoyl-sn-glycero-3-phosphocholine dithiothreitol

glutamic acid

permittivity

vacuum permittivity

extracellular

Escherichia coli

electro-osmotic flow

frequency

cutoff frequency

full width half maximum

glycine

conductance

open channel conductance

glutamic acid

glycine

membrane conductance

membrane conductance with open channel

giant lamellar vesicle

4-(2-hydroxyethyl)-1-piperazineethanesulfonic

acid

current

inner mitochondrial membrane

interquartile width

indium tin oxide

【Ump Local dEconvolution Segmentation filter

lysine

dissociation rate

association rate

'maltoporin'

N,N-Dimethyldodecylamine N-oxide

lysine 
M

M

MD

MECA

MOMP

$\mathrm{n}$

$\mathrm{NADH}$

Ngo

Nme

$\mathrm{OM}$

OMM

OMP

p. a.

PDB

PhoE

pK

pKa

$P_{\text {o }}$

POPC

PP

PTFE

$\mathrm{R}$

$R$

RESP

$R_{\mathrm{m}}$

$R_{\mathrm{m}, \mathrm{c}}$

RMSD

rpm

$\sigma$

SDS-PAGE

sel

SPC/E

$t$ monomer

molecular mass

molecular dynamics

micro electrode cavity array

mitochondrial outer membrane permeabilization

number of events

nicotinamide adenine dinucleotide

Neisseria gonorrhea

Neisseria meningitidis

outer membrane

outer mitochondrial membrane

outer membrane protein

pro analysi

protein data bank

phosphoporin

negative logarithm of the dissociation constant $\mathrm{K}$

negative decadic logarithm of acid dissociation con-

stant

open probability

1-palmitoyl-2-oleoyl-sn-glycero-3-phosphocholine

periplasmic

polytetrafluoroethylene

arginine

resistance

restrained electrostatic potential

membrane resistance

membrane resistance with open channel

root mean square deviations

rounds per minute

standard deviation

sodium dodecyl sulfate polyacrylamide gel electro-

phoresis

selectivity

extended simple point charge

time 
$\mathrm{T}$

$\tau$

TEMED

TOM

$t_{\mathrm{R}}$

$\tau_{\mathrm{R}}$

TRIS

$U$

$U_{\text {inf }}$

$U_{\mathrm{m}}$

$Q$

$(v / v)$

$\mathrm{Wt}$

$(w / v)$

$(w / w)$ trimer

time constant

$\mathrm{N}, \mathrm{N}, \mathrm{N}$ ',N'-tetramethylethylendiamine

translocase of the outer mitochondrial membrane

rise time

residence time

tris(hydroxymethyl)aminomethane

voltage

equilibrium voltage

transmembrane voltage/potential

charge

concentration in volume/volume percent

wild type

concentration in weight/volume percent

concentration in weight/weight percent

\subsection{Chemicals and consumables}

acetone p.a.

acrylamide/bisacrylamide

agarose NEEO

ampicillin sodium salt

APS

borosilicate chips NPC-1

bromphenol blue

chloroform

cholesterol

Coomassie-Brilliant-Blue G-250

decane

DPhPC

DTT

ethannol p.a.
Carl Roth GmbH, Karlsruhe, GER

Carl Roth GmbH, Karlsruhe, GER

Sigma Aldrich, Taufkirchen, GER

Carl Roth GmbH, Karlsruhe, GER

Sigma Aldrich, Taufkirchen, GER

Nanion Technologies, München, GER

Carl Roth GmbH, Karlsruhe, GER

VWR, Darmstadt, GER

Sigma-Aldrich, Taufkirchen, GER

Fluka Chemie GmbH, Seelze, GER

Sigma-Aldrich, Taufkirchen, GER

Avanti Polar Lipids, Alabaster, USA

Sigma Aldrich, Taufkirchen, GER

VWR International, Darmstadt, GER 
glass slides

glycerol

hematocrit capillaries

HEPES

hydrochloric acid

isopropanol

ITO slides

LDAO

MECA chips

methanol

Parafilm ${ }^{\circledR}$

potassium chloride

potassium hydroxide

PTFE foil, DF100 cast film

SDS

sodium chloride

sodium hypochlorite

sucrose

TEMED

TRIS
Glasbearbeitung Henneberg \& Co., Martinroda, GER

Merck, Darmstadt, GER

VWR, Darmstadt, GER

Carl Roth GmbH, Karlsruhe, GER

Merck, Darmstadt, GER

Merck, Darmstadt, GER

Präzisions Glas \& Optik GmbH, Iserlohn, GER

Sigma-Aldrich, Taufkirchen, GER

Ionera Technologies GmbH, Freiburg, GER

Carl Roth GmbH, Karlsruhe, GER

Pechiney Plastic Packaging, Chicago, IL, USA

Merck, Darmstadt, GER

Merck, Darmstadt, GER

Saint-Gobain Performance Plastics, Rochdale, UK

AppliChem GmbH, Darmstadt, GER

Merck, Darmstadt, GER

VWR, Darmstadt, GER

Carl Roth GmbH, Karlsruhe, GER

Sigma Aldrich, Taufkirchen, GER

Carl Roth GmbH, Karlsruhe, GER

\subsection{Devices}

A/D-converter Digidata 1322

amplifier Axopatch 200 B

frequency generator: Agilent

$3220 \mathrm{~A}$

Hamilton ${ }^{\circledR}$ syringes

headstage CV 203BU

lamp SUN-1

Mini-PROTEAN@ Multi-Casting

chamber
Axon Instruments, Union City, CA, USA

Axon Instruments, Union City, CA, USA

Agilent Technologies, Santa Clara, CA USA

Sigma-Aldrich, Taufkirchen, GER

Axon Instruments, Union City, CA, USA

Warner Instruments LLC, Hamden, CT, USA

Bio-Rad Laboratories, Inc., Hercules, CA, USA 
microliter pipettes

microscale Sartorius CP225D

NanoDrop 2000c

Orbit 16

Osmometer: Osmomat 3000

pH-meter: Calimatic 766

Port-a-Patch

stirrer SPIN-2

thermomixer Compact

ultrasonic bath: Sonorex RK $255 \mathrm{H}$
Eppendorf AG, Hamburg, GER

Sartorius, Göttingen, GER

Thermo Scientific, Wilmington, USA

Nanion Technologies, München, GER

Gonotec, Berlin, GER

Knick, Berlin, GER

Nanion Technologies, München, GER

Warner Instruments LLC, Hamden, CT, USA

Eppendorf AG, Hamburg, GER

Badelin, Berlin, GER

\subsection{Software}

Clampex 10.4.0.36

Clampfit 10.4.0.36

EDR software 3.3.8

MATLAB R2014a

Origin Pro 8.5G

PatchControl
Molecular Devices, Sunnyvale, CA, USA

Molecular Devices, Sunnyvale, CA, USA

elements SRL, Cesena (FC), Italy

The MathWorks, Inc., Natick, MA, USA

OriginLab Corporation, Northampton, MA, USA

Nanion Technologies, München, GER

\subsection{MATLAB scripts}

\subsubsection{Point amplitude histograms}

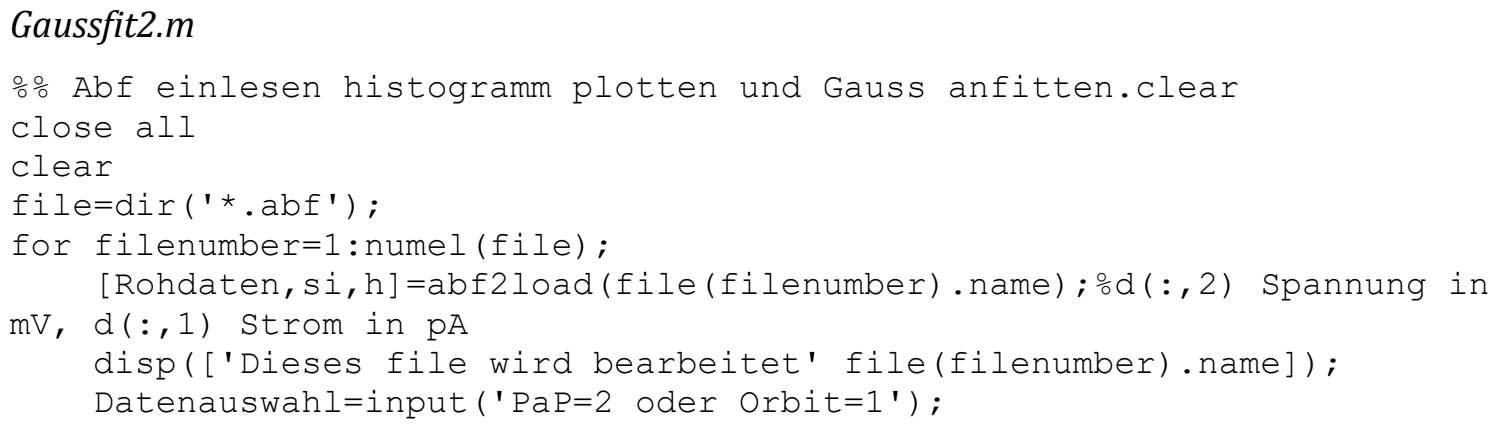




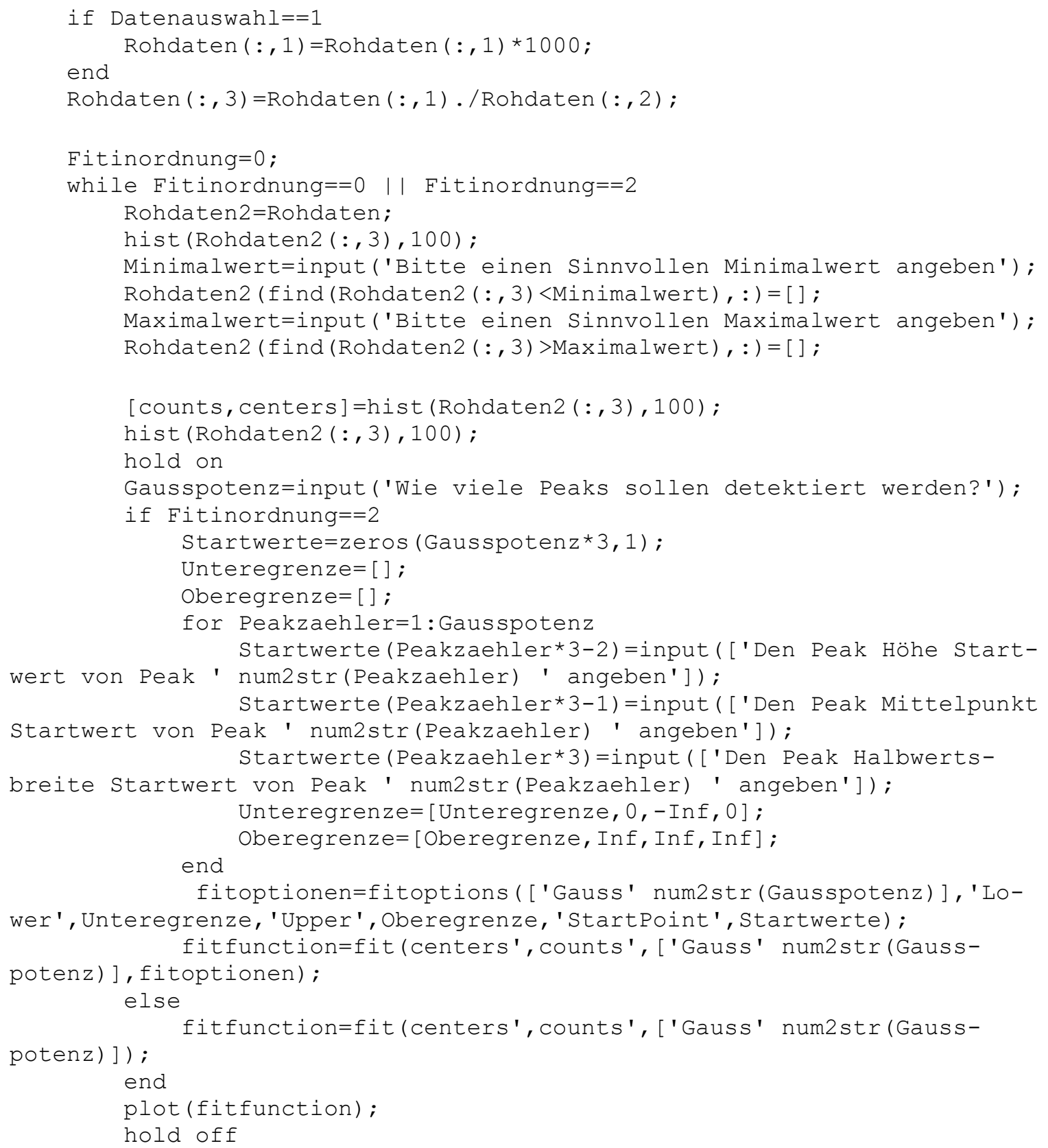

Fitinordnung=input('Ist der Fit in Ordnung? Ja=1, Nein=0, Manuelle Peakstartwerteingabe=2'); end xlabel ('G / $\mathrm{nS}$ '); ylabel ('Counts'); saveas (gca, [file(filenumber). name (1:end-4) '_histogramm.png'], 'png'); Flaeche. Komplett=integrate (fitfunction, Maximalwert, Minimalwert);

fitvalues=coeffvalues (fitfunction); PeakMittelpunkte=fitvalues $(2: 3:$ end $)$; PeakMittelpunkte=sort (PeakMittelpunkte) PeakMittelpunkte $=[-$ PeakMittelpunkte $(1)+2 *$ Minimalwert, PeakMittelpunkte, 2*Maximalwert-PeakMittelpunkte (end) ] ;

for $k=1$ : Gausspotenz 


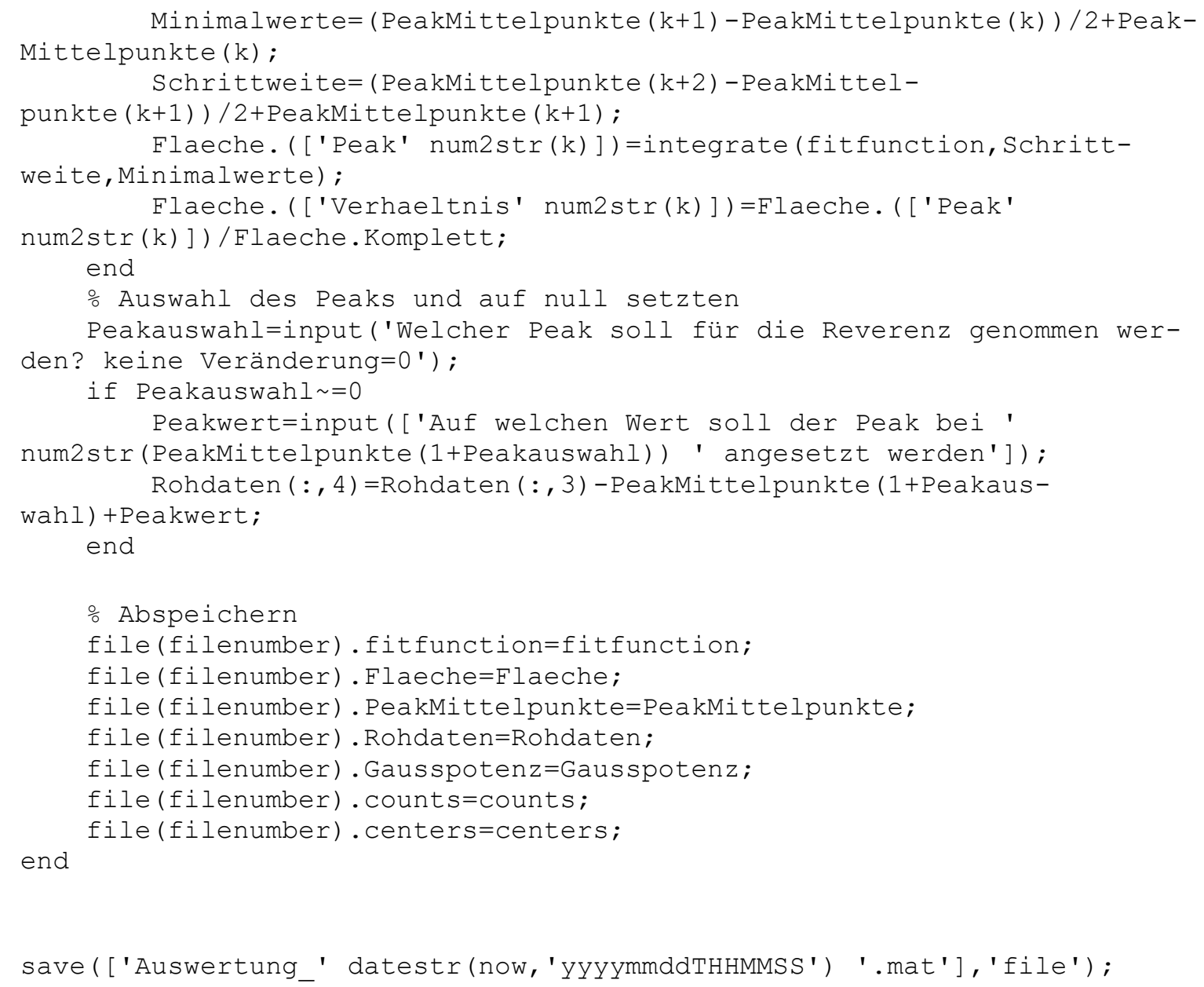

\section{GaussfitN.m}

응 Normierte Daten als Histogramm plotten und Gaussverteilung anpassen

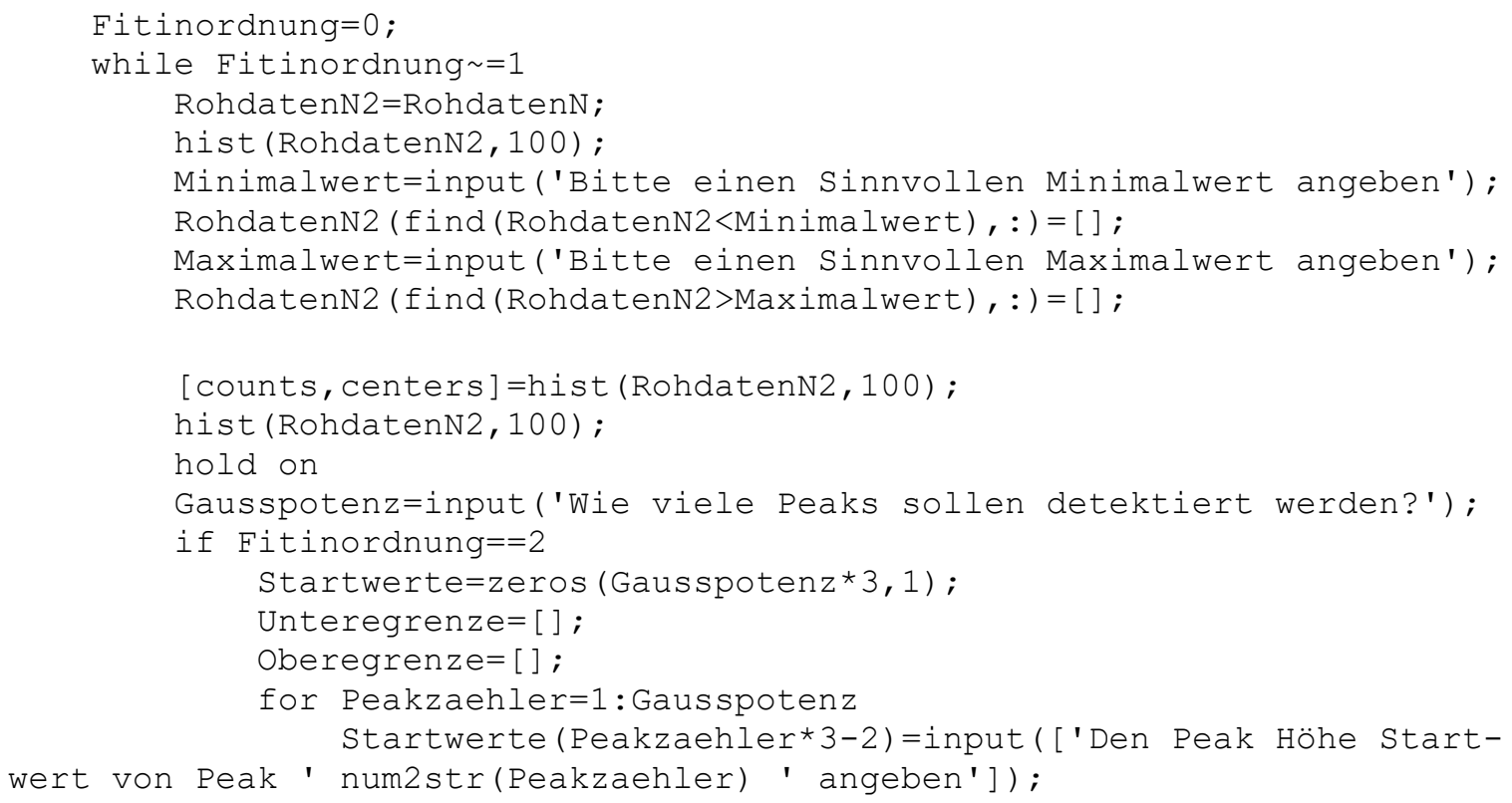


Startwerte (Peakzaehler*3-1) =input ( ['Den Peak Mittelpukt Startwert von Peak ' num2str(Peakzaehler) ' angeben']); Startwerte (Peakzaehler*3)=input ( ['Den Peak Halbwertsbreite Startwert von Peak ' num2str(Peakzaehler) ' angeben']); Unteregrenze $=[$ Unteregrenze, $0,-\operatorname{Inf}, 0]$; end Oberegrenze=[Oberegrenze, Inf, Inf, Inf] ;

fitoptionen=fitoptions (['Gauss' num2str(Gausspotenz)], 'Lower', Unteregrenze, 'Upper', Oberegrenze, 'StartPoint', Startwerte) ;

fitfunction=fit (centers', counts', ['Gauss' num2str(Gauss-

potenz) ], fitoptionen); else

potenz)] );

fitfunction=fit(centers', counts', ['Gauss' num2str(Gauss-

end

plot (fitfunction);

hold off

Fitinordnung=input('Ist der Fit in Ordnung? Ja=1, Nein=0, Manu-

elle Peakstartwerteingabe=2');

end

xlabel ('G / nS');

ylabel ('Counts');

saveas (gca, ['Auswertung_' datestr(now, 'yyyymmddTHHMMSS') '_histo-

gramm.png'], 'png');

Flaeche.Komplett=integrate (fitfunction, Maximalwert, Minimalwert);

fitvalues=coeffvalues (fitfunction);

PeakMittelpunkte=fitvalues $(2: 3:$ end $)$;

PeakMittelpunkte=sort (PeakMittelpunkte)

PeakMittelpunkte $=[-$ PeakMittelpunkte $(1)+2 *$ Minimalwert, PeakMittel-

punkte, $2 *$ Maximalwert-PeakMittelpunkte (end) ] ;

for $\mathrm{k}=1$ : Gausspotenz

Minimalwerte $=($ PeakMittelpunkte $(k+1)$-PeakMittelpunkte $(k)) / 2+$ PeakMittelpunkte (k);

Schrittweite $=($ PeakMittelpunkte $(k+2)$-PeakMittel-

punkte $(\mathrm{k}+1)) / 2+$ PeakMittelpunkte $(\mathrm{k}+1)$;

Flaeche. (['Peak' num2str(k)])=integrate(fitfunction, Schrittweite, Minimalwerte);

Flaeche. (['Verhaeltnis' num2str(k)])=Flaeche. (['Peak'

num2str(k) ])/Flaeche.Komplett;

end

o Auswahl des Peaks und auf null setzten

Peakauswahl=input('Welcher Peak soll für die Reverenz genommen werden? keine Veränderung=0') ;

if Peakauswahl =0

Peakwert=input (['Auf welchen Wert soll der Peak bei'

num2str(PeakMittelpunkte (1+Peakauswahl)) ' angestzt werden']);

Rohdaten $(:, 4)=$ Rohdaten $(:, 3)$-PeakMittelpunkte $(1+$ Peakaus-

wahl) +Peakwert;

end

\% Abspeichern

file2.fitfunction=fitfunction;

file2.Flaeche=Flaeche;

file2. PeakMittelpunkte=PeakMittelpunkte;

file2. RohdatenN=RohdatenN;

file2. Rohdaten=Rohdaten;

file2. Gausspotenz=Gausspotenz; 
file2. counts=counts;

file2. centers=centers;

save(['Auswertung_' datestr(now, 'yyyymmddTHHMMSS') '.mat'], 'file2');

\subsubsection{Noise analysis}

\section{PorB.m}

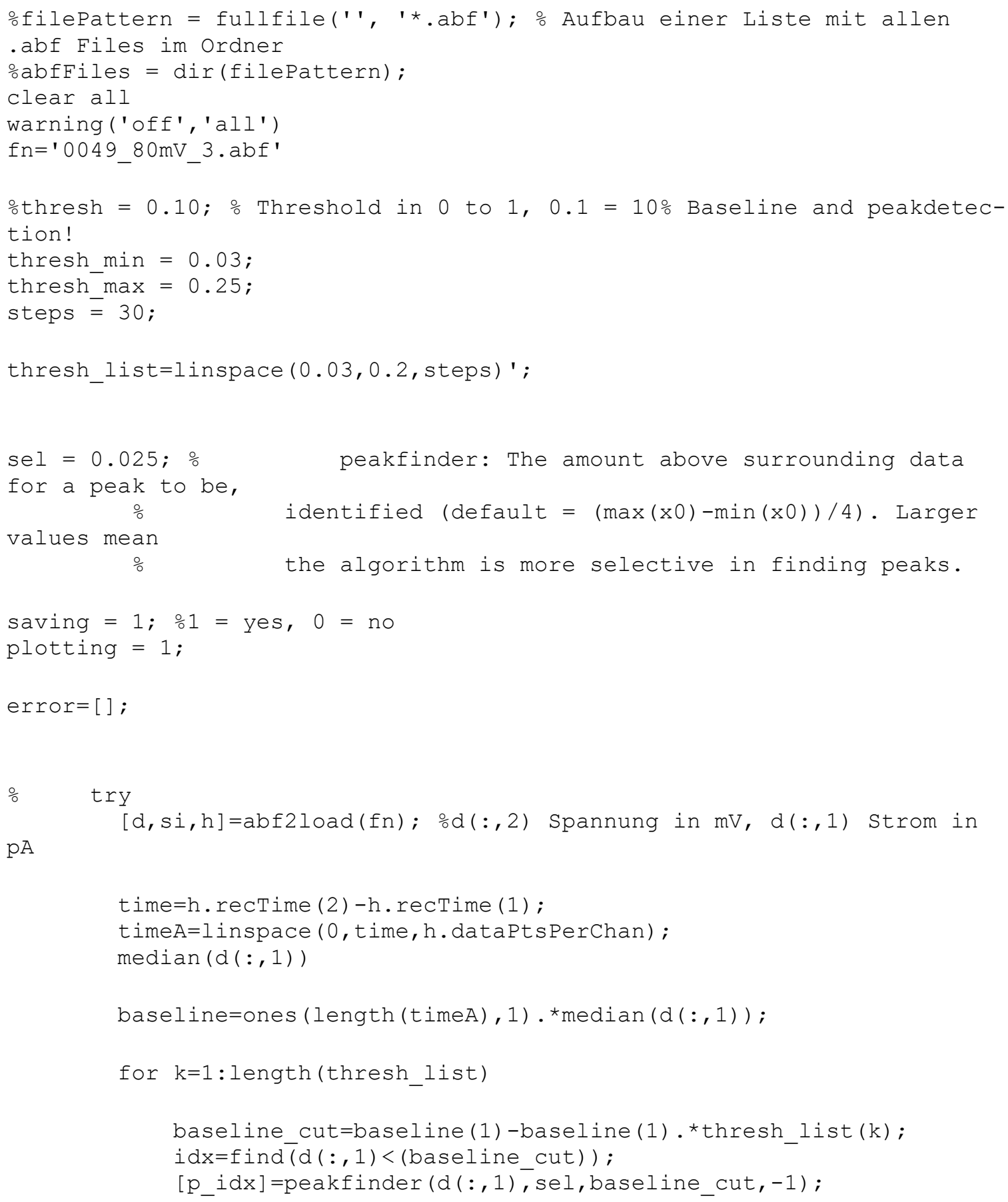




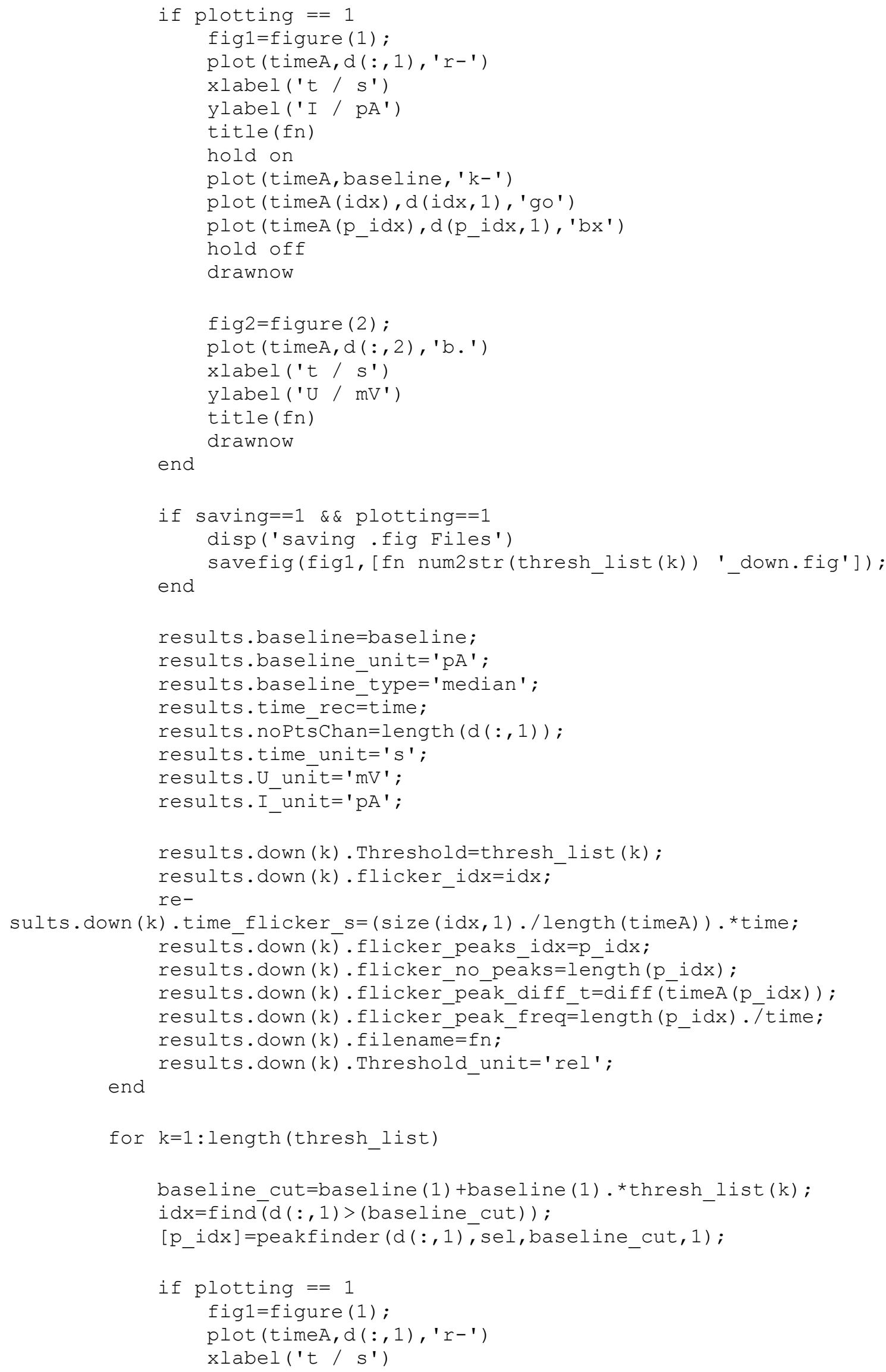




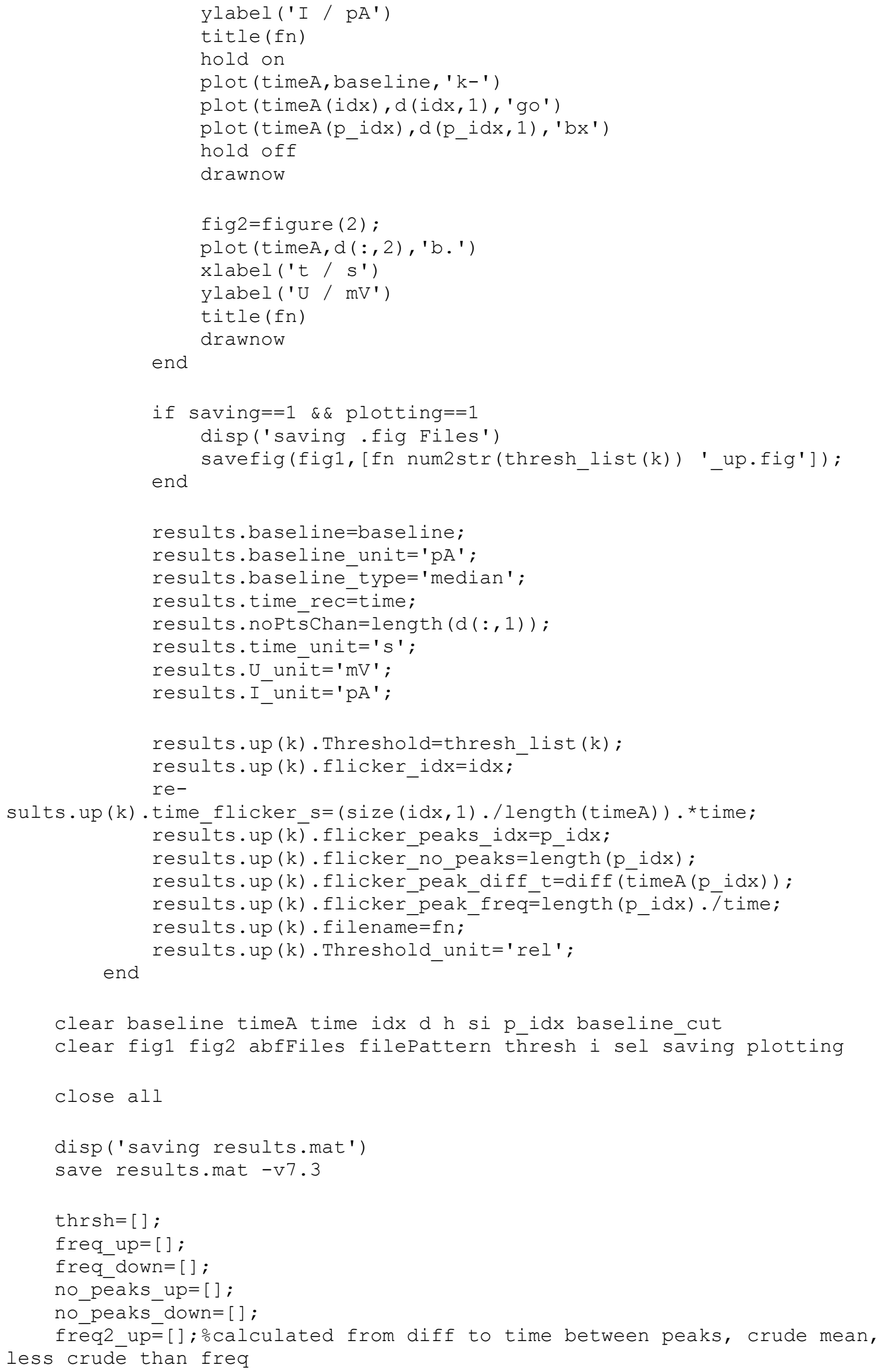




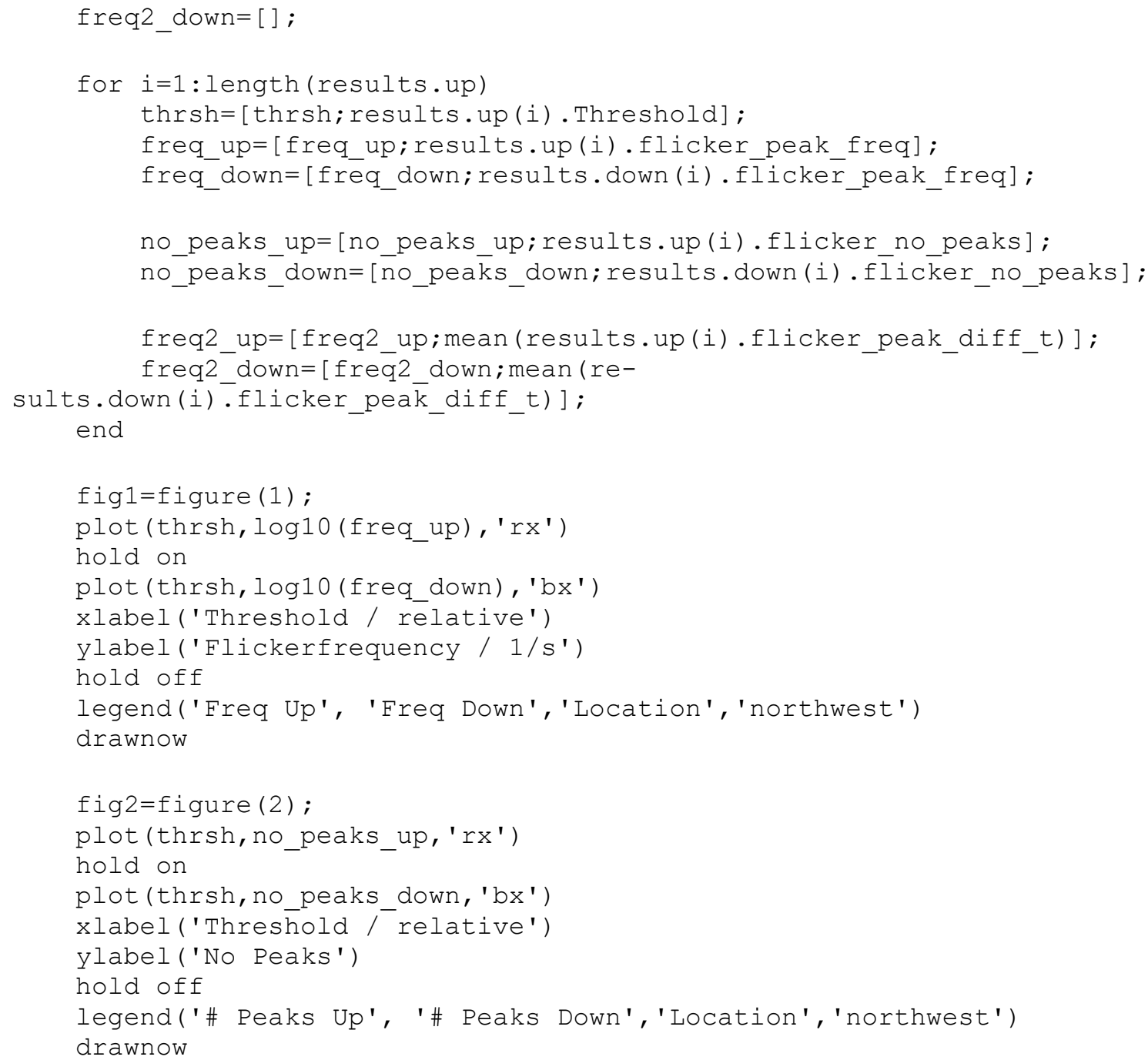




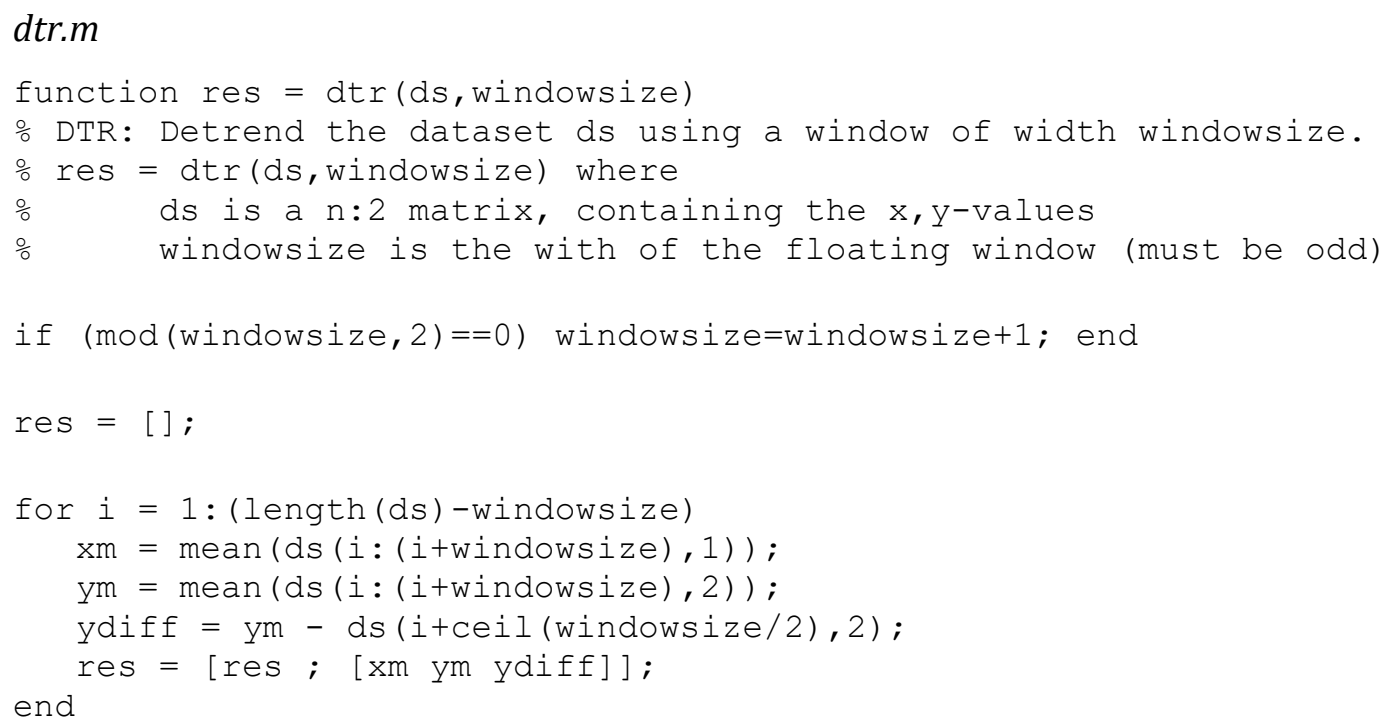




\section{Curriculum vitae}

\section{Annika Bartsch}

Born on April 5th, 1989 in Mainz

Citizenship: German

\section{Education}

since $12 / 2013$

Ph.D. thesis at the Institute of Biomolecular and Organic Chemistry, Georg-August-University Göttingen (Prof. Dr. C. Steinem)

Title of thesis: Influence of point mutations on the electrophysiological properties of a bacterial porin and its interaction with a $\beta$-lactam antibiotic.

10/2011 - 10/2013 Graduate studies in chemistry at the Georg-August-University Göttingen (Prof. Dr. C. Steinem)

Title of the Master's thesis: Electrophysiological characterization of the influence of the membrane-peptide-interaction on the function of ion channels.

10/2012 - 3/2013 Erasmus stay at the École Normale Supérieure, Paris.

Title of research thesis: Influence of Cell Penetrating Peptides on phospholipid flip/flop.

$10 / 2008-07 / 2011 \quad$ Undergraduate studies in chemistry at the Georg-August-University Göttingen (Prof. Dr. C. Steinem)

Title of the Bachelor's thesis: Atomic Force Microscope imaging of Ezrin on solid supported bilayers.

06/2008 Abitur at the Friedrich-Gymnasium, Freiburg.

\section{Scientific contributions}

2015

$10^{\text {th }}$ European Biophysics Congress, Dresden. Electrophysiological characterization of the bacterial porin PorB and its mutant. 

physiological investigation of biomolecules inducing membrane permeability. saing, C. Mansuy, F. Burlina, S. Lavielle, The efficacies of Cell-Penetrating Peptides in accumulating in large unilamellar vesicles depend on their ability to form inverted micelles, ChemBioChem 2014, $15,884-891$. 


\section{Danksagung}

An erster Stelle bedanke ich mich bei Prof. Dr. Claudia Steinem für die Betreuung meiner Doktorarbeit und die vielen fachlichen Diskussionen, die mein Projekt vorangebracht haben.

Ich danke den Mitgliedern meines Thesis Committees, Prof. Dr. Ulf Diederichsen und Prof. Dr. Michael Meinecke, für die Unterstützung meiner Arbeit und die wertvollen Ratschläge.

Besonderer Dank gilt meinen Kooperationspartnern Mikio Tanabe und Christof Kattner für die Bereitstellung der Proteine und den Anstoß für mein Projekt, Prof. Dr. Axel Munk und Florian Pein für die Entwicklung und Anwendung von JULES, sowie Dr. Ulrich Zachariae und Salomé Llabrés für die Durchführung der MD Simulationen. Großer Dank geht an Prof. Dr. Michael Meinecke und Niels Denkert für die Unterstützung beim Aufbau des BLM Setups.

Ingo und Martin danke ich ganz herzlich für viele fachliche Diskussionen, ihre MATLABkenntnisse und die großartige moralische Unterstützung. Ingo gilt insbesondere großer Dank für das Voranbringen meiner Datenauswertung. An dieser Stelle danke ich auch Jeremias für seine tatkräftige Unterstützung in Form eines MATLAB-Skriptes.

Jutta und Micha danke ich für die Unterstützung durch diverse Arbeiten im Labor. Für die stete Hilfe in organisatorischen Belangen bedanke ich mich beim gesamten Sekretariat.

Meinen Bachelorstudenten Hannah, Svenja und Linus, sowie meinem Masterstudenten Markus danke ich für die vielen Experimente, die meine Arbeit bereichert haben.

Großer Dank geht an alle, die meine Arbeit Korrektur gelesen haben. Herzlicher Dank geht an den gesamten Arbeitskreis für die tolle Arbeitsatmosphäre, die Messzeiten, Grillabende und Kegelrunden, die mich immer wieder aufs Neue motiviert haben. Insbesondere danke ich der Frühstücksrunde für einen guten Start in den Tag und meinen Mitleidenden im Schreibraum, Miri und Jan, für auflockernde Momente und Ratschläge.

Allen ehemaligen und aktuellen „Göttinger Chaoten“ möchte ich ganz herzlich für die unvergessliche Studien- und Promotionszeit danken. Schön, dass wir auch trotz kleiner und großer geografischer Distanzen weiterhin Kontakt halten!

Von ganzem Herzen danke ich meinen Eltern und meiner Schwester! Ohne den familiären Rückhalt, das Vertrauen in mich und die stets offenen Ohren für meine Probleme, wäre ich nie so weit gekommen. Ich habe euch lieb!

Aus tiefstem Herzen danke ich Sascha dafür, dass er seit so vielen Jahren in jeder Lebenslage für mich da ist, mir Mut macht und mir meine Sorgen nimmt. Ohne dich wäre ich nicht vollständig! Das gilt auch für meine kleine Emilie, die nur einmal Lächeln muss, um meinen gesamten Tag zu retten. Ich freue mich auf unsere gemeinsame Zukunft! 
